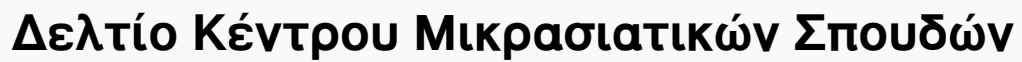

Tóp. 10 (1993)

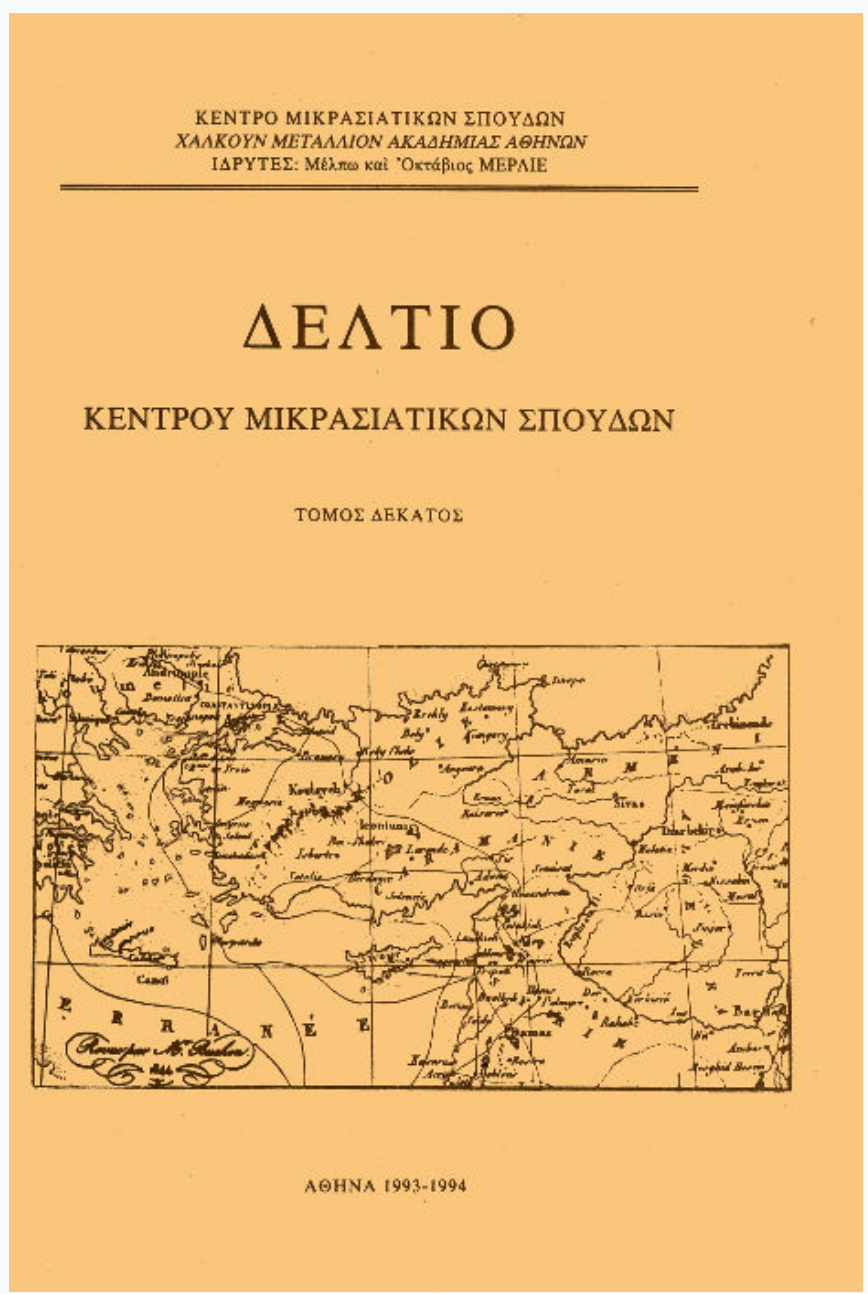

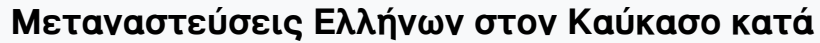 Tov 190 aı́́va}

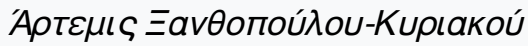

doi: $10.12681 /$ deltiokms.97

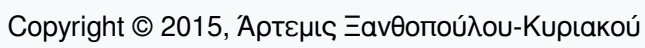

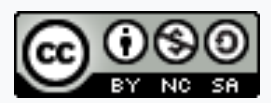

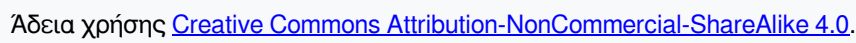

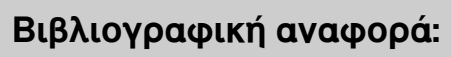

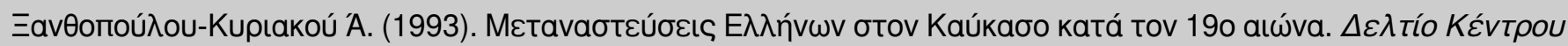

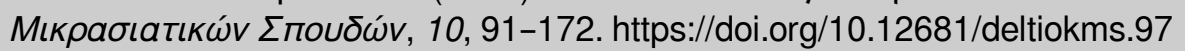




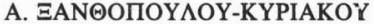

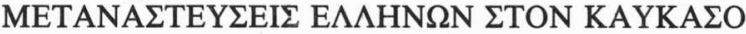 KATA TON 19o AI $\Omega$ NA}

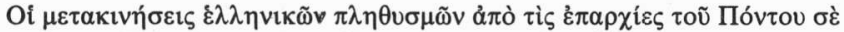

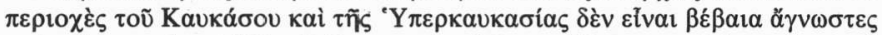

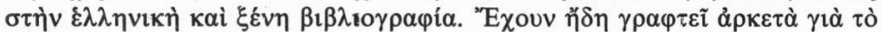

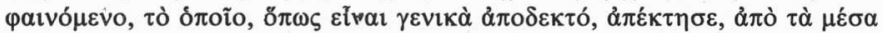

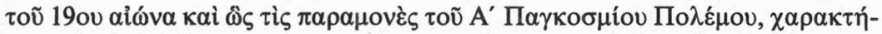

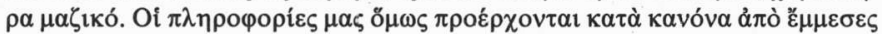

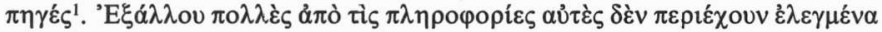

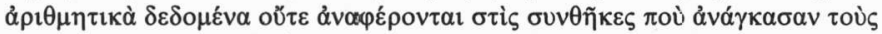

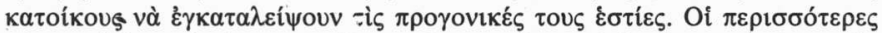

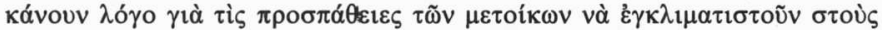

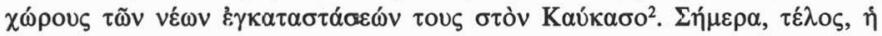

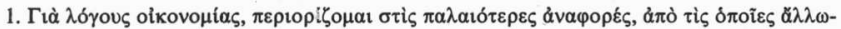

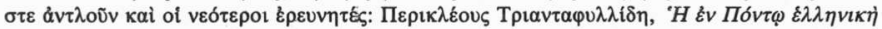

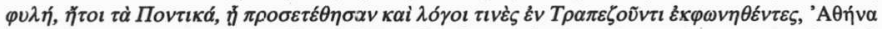

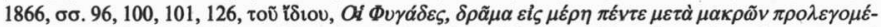

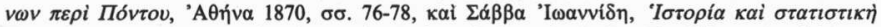

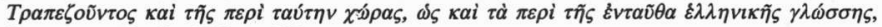
К $\omega \nu \sigma \tau \alpha v \tau \iota v o u ́ \pi 0 \lambda \eta 1870, \sigma .253$.

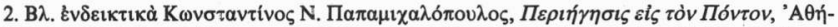

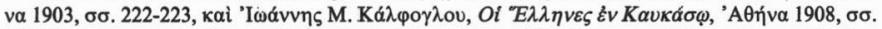

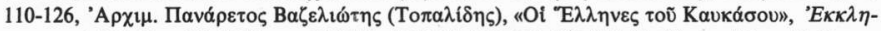

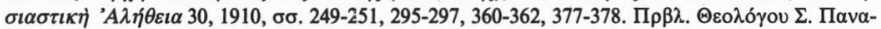

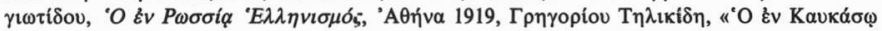

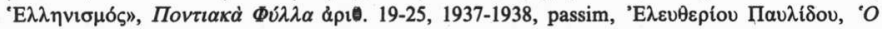

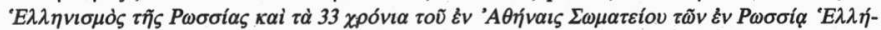

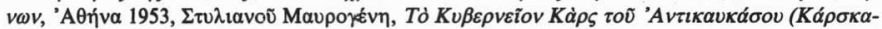
$\gamma t \alpha$ "O

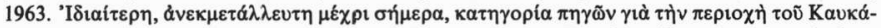

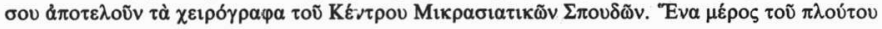




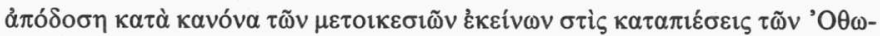

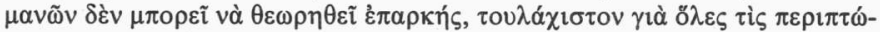

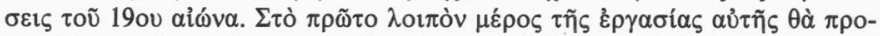

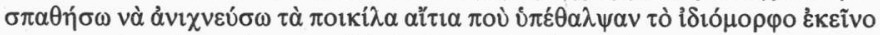

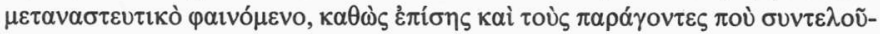

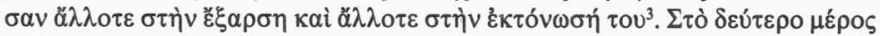

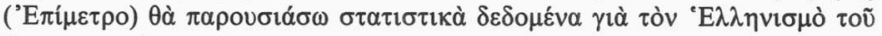

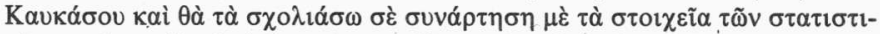

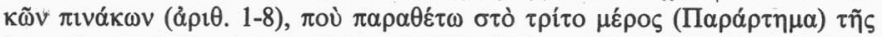

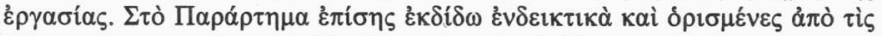

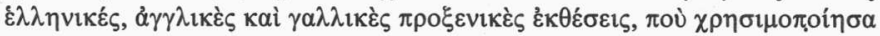

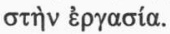

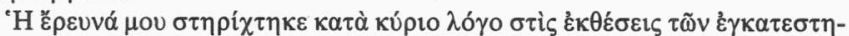

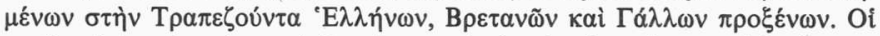

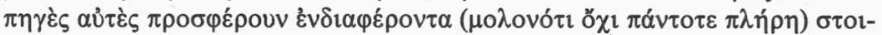

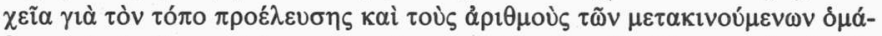

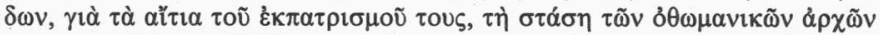

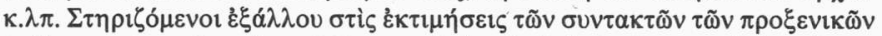

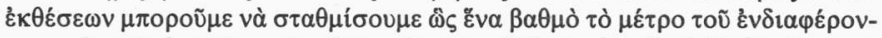

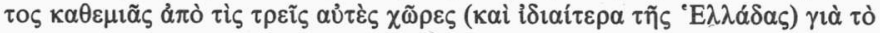

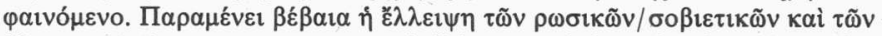

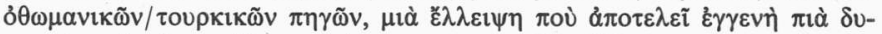

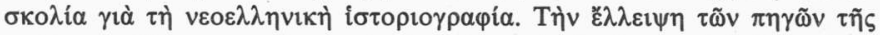

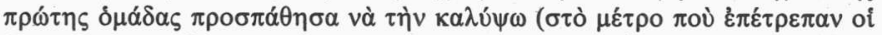

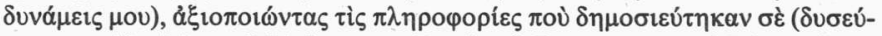

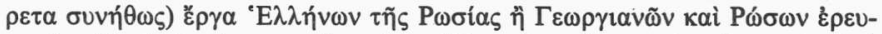

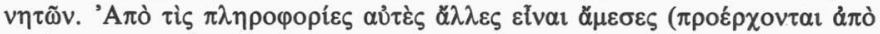

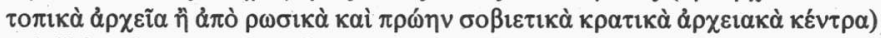

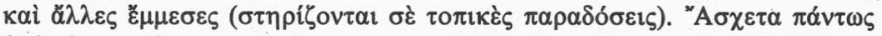

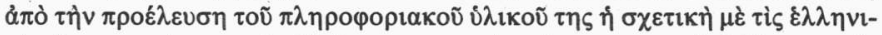

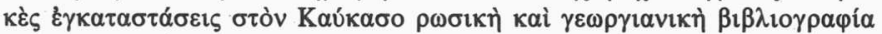

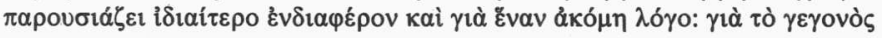

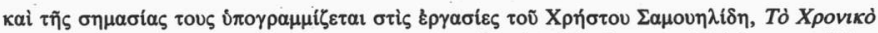

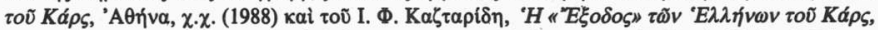

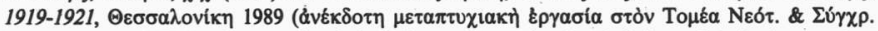

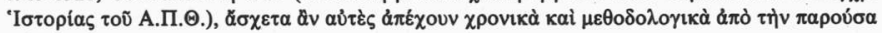

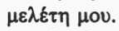

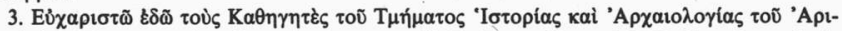

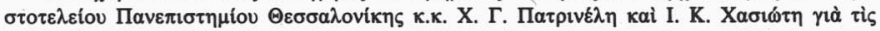

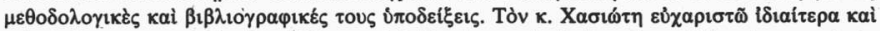

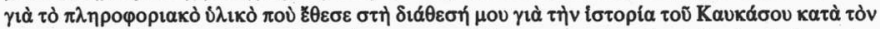

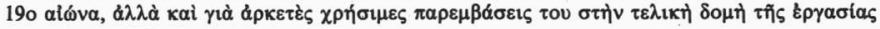

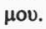




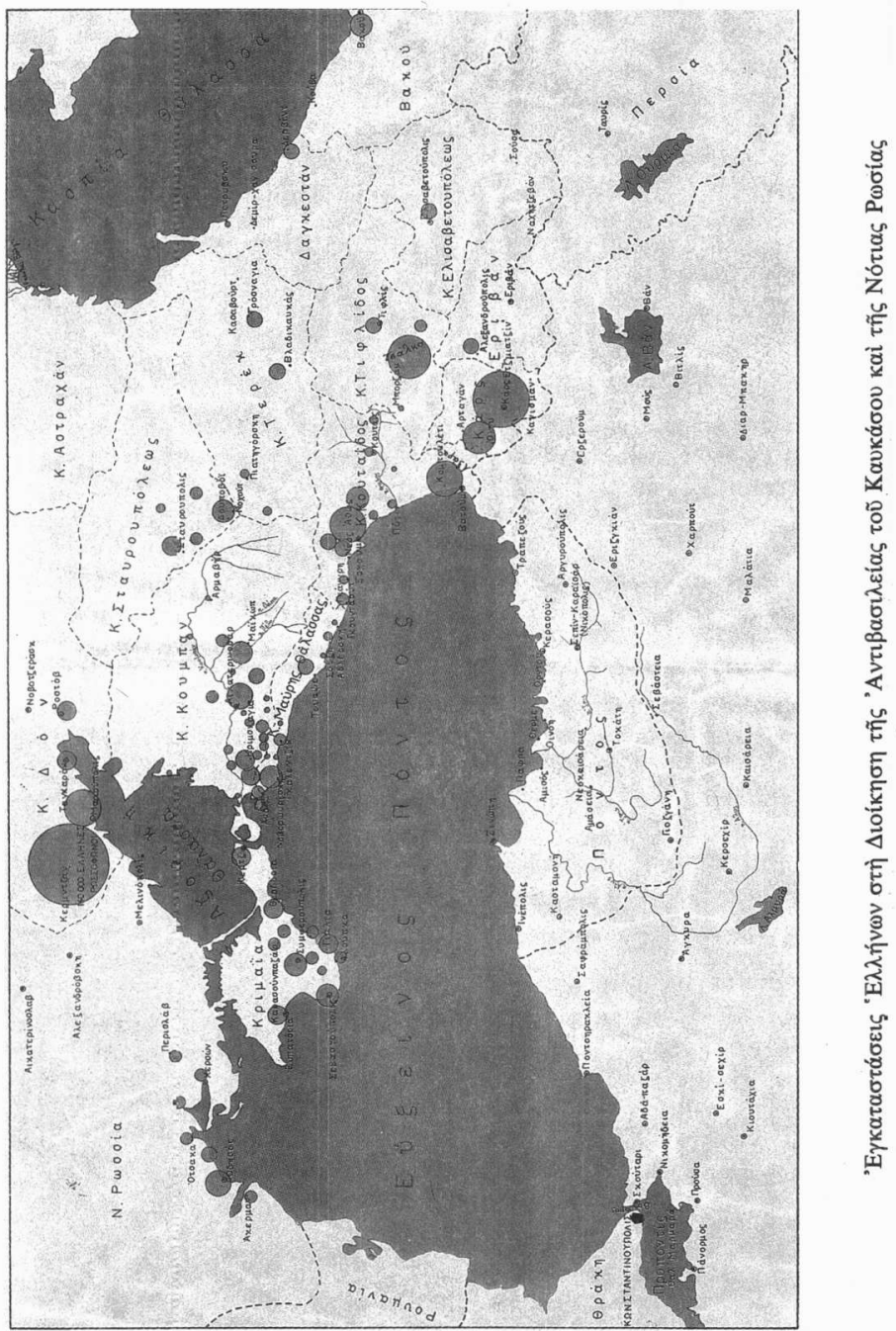




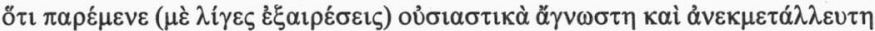

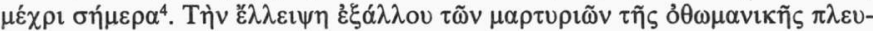

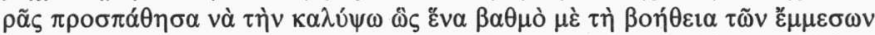

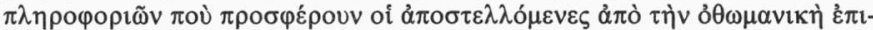

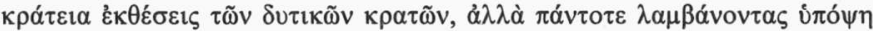

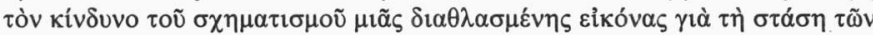

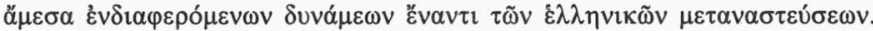

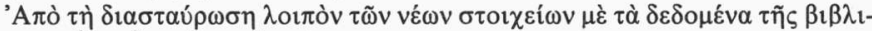

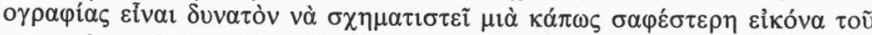

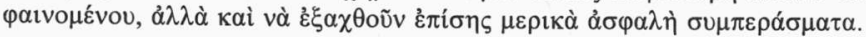

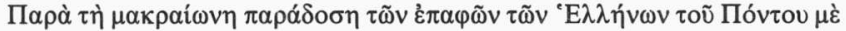

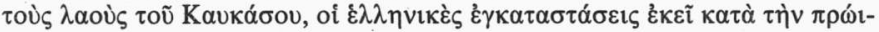

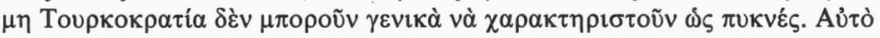

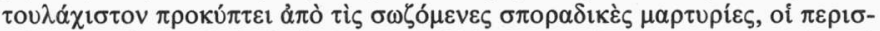

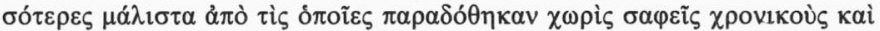

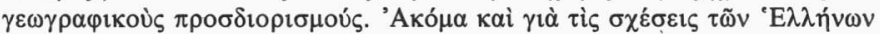

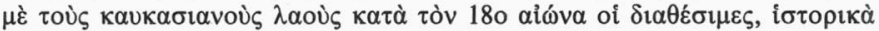

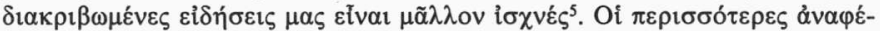

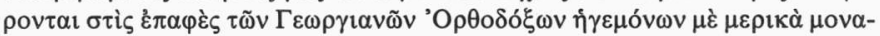

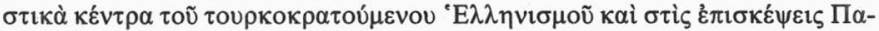

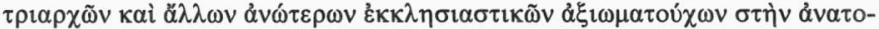

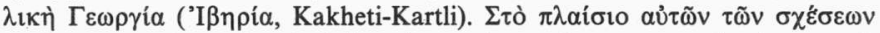

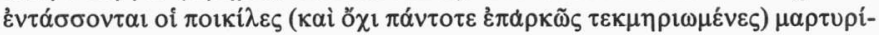

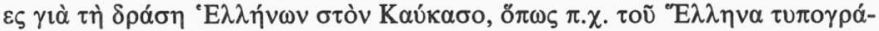

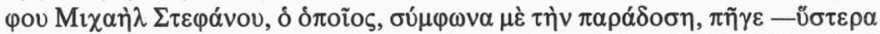

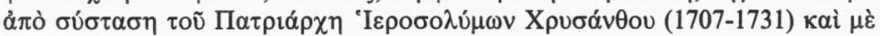

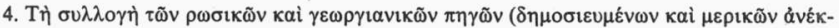

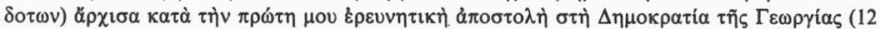

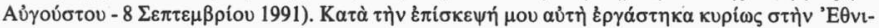

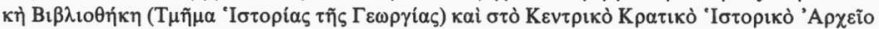

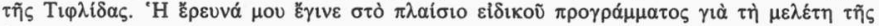

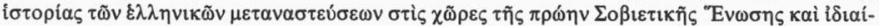

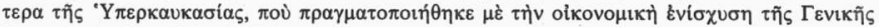

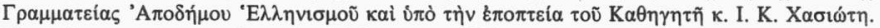

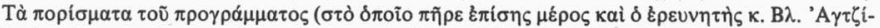

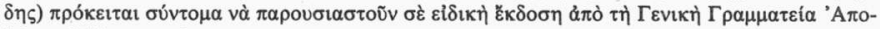

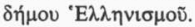

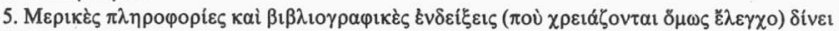

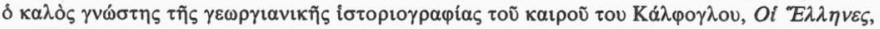
бб. 91-98. 


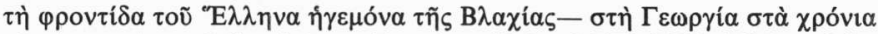

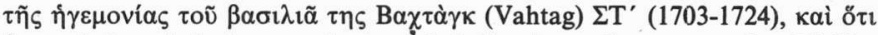

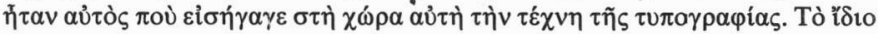

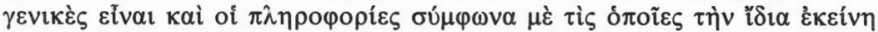

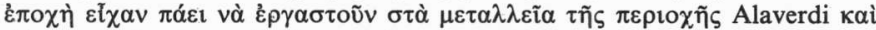

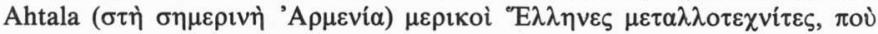

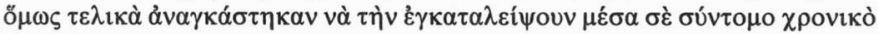
$\delta i \alpha ́ \sigma \tau \eta \mu \alpha^{6}$.

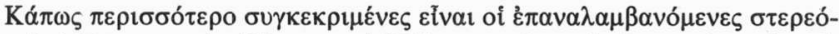

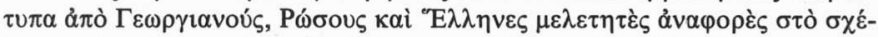

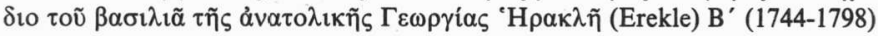

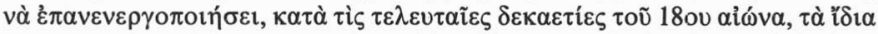

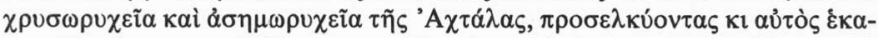

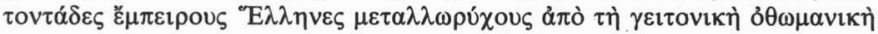

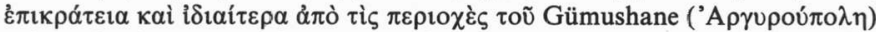

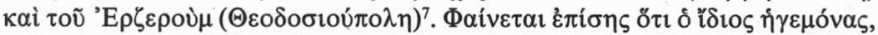

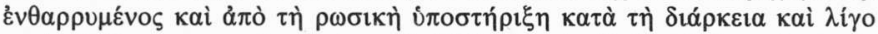

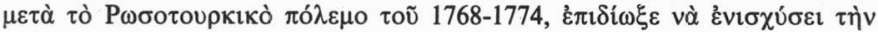

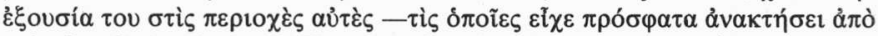

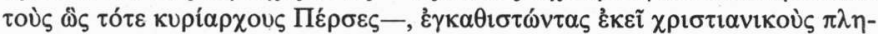

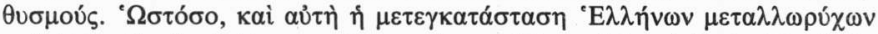

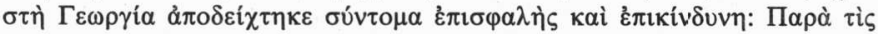

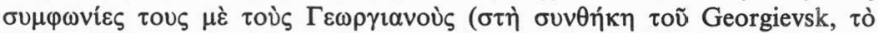

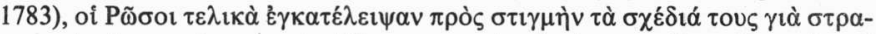

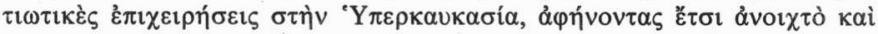

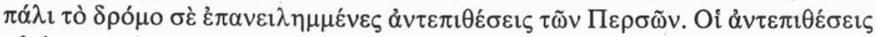

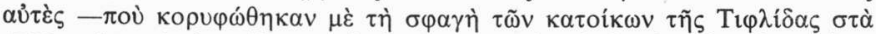

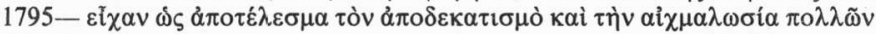

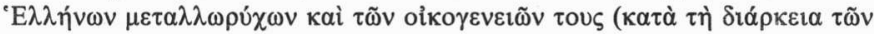

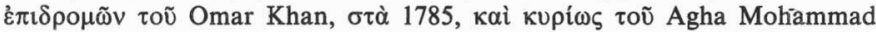

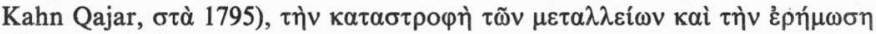
$\tau \tilde{\eta} \varsigma \pi \varepsilon \rho 10 \chi \tilde{\eta} \varsigma^{8}$.

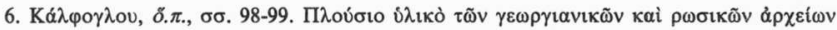
$\sigma \chi \varepsilon \tau$

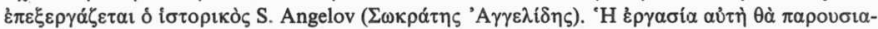

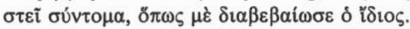

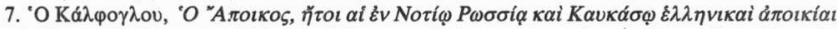

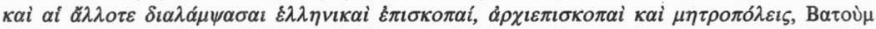

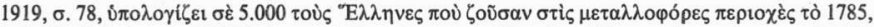

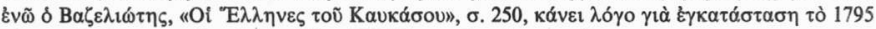

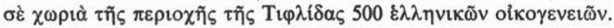

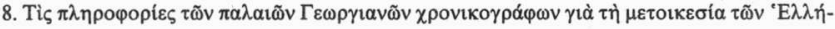




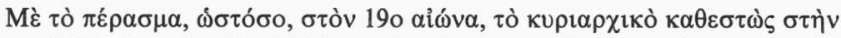

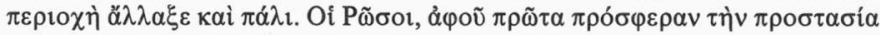

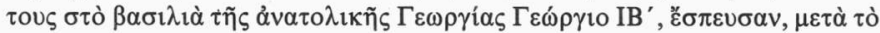

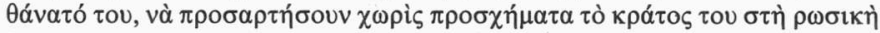

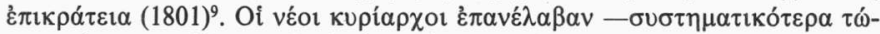

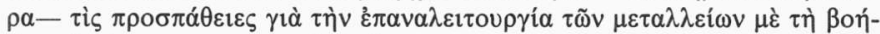

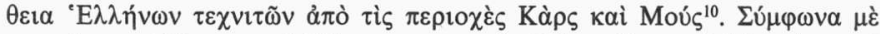

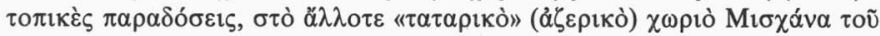

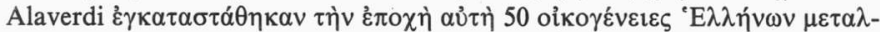

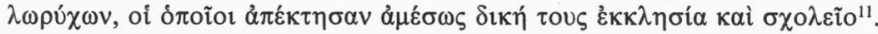

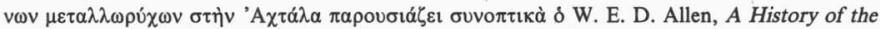

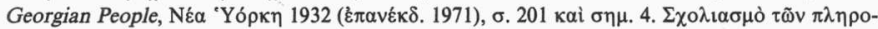

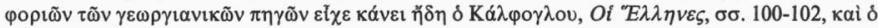

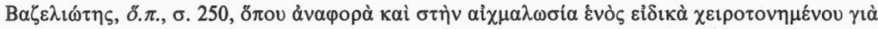

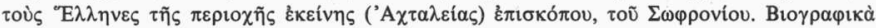

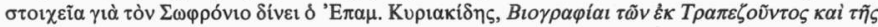

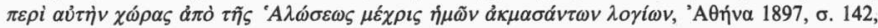

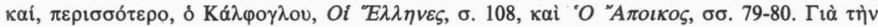

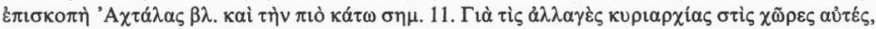
$\beta \lambda$. George A. Bournoutian, Eastern Armenia in the Last Decades of Persian Rule, 1807-1828. A Political and Socioeconomic Study of the Khanate of Erevan on the Eve of the Russian Conquest,

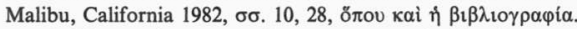

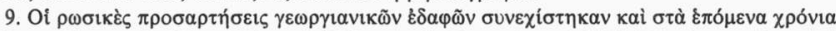

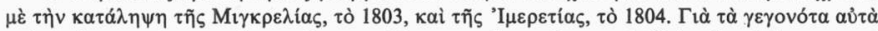

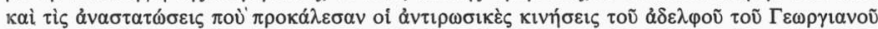

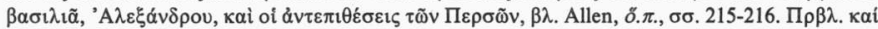

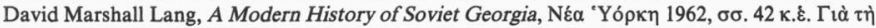

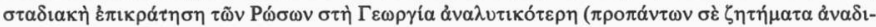

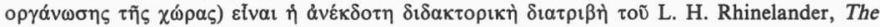

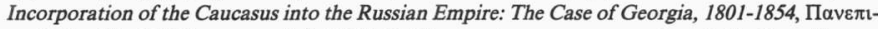

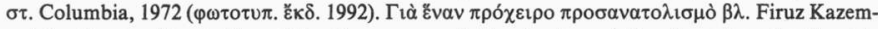

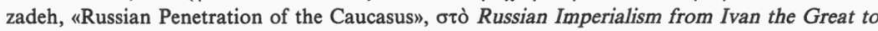
the Revolution, $\varepsilon \pi \iota \mu$. T. Hunczak, N. Brunswick, N.J., 1974, бб. 239-263.

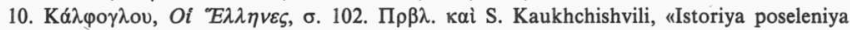

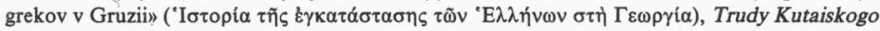

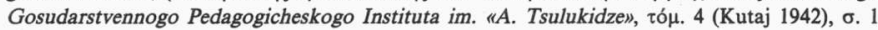
(¿̊ór.).

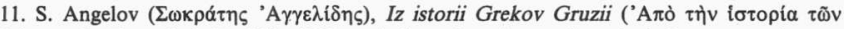

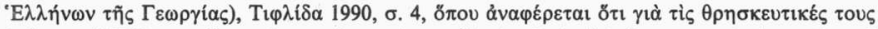

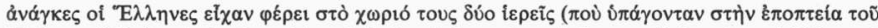

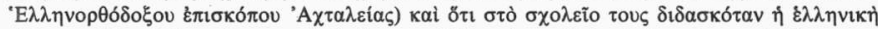

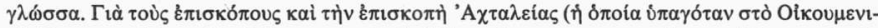

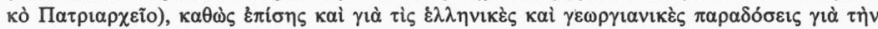

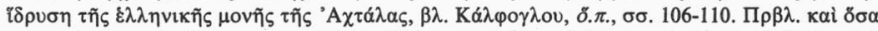

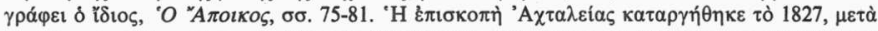

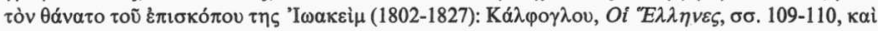

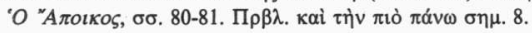




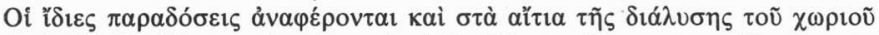

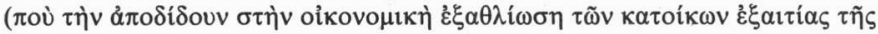

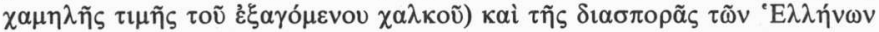

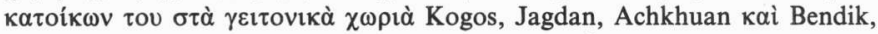

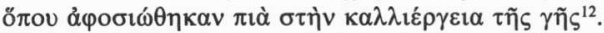

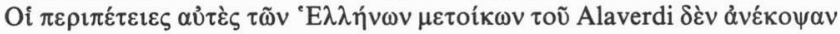

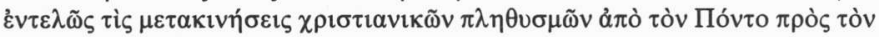

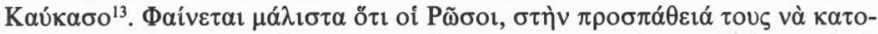

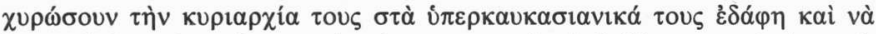

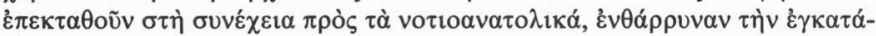

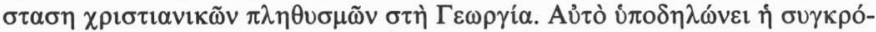

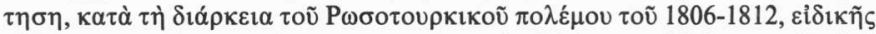

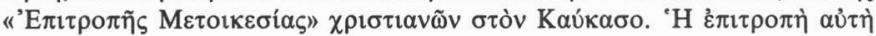

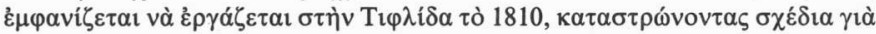

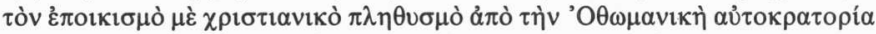

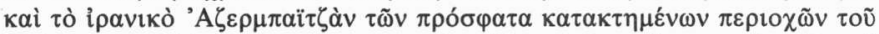

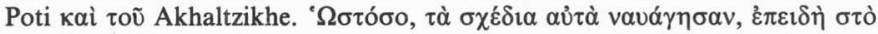

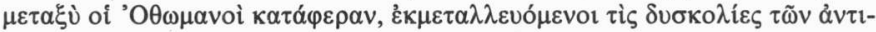

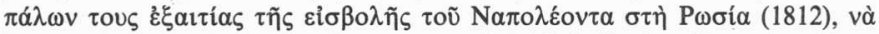

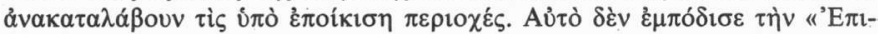

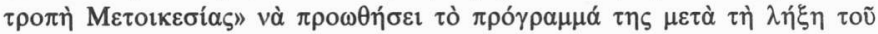

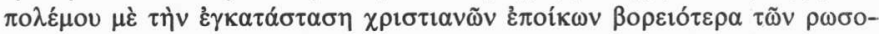

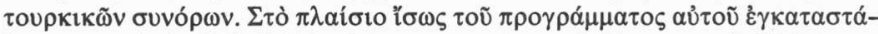

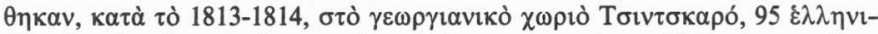

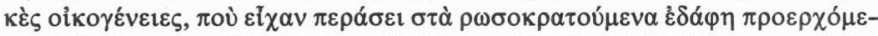

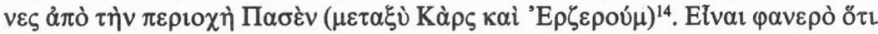

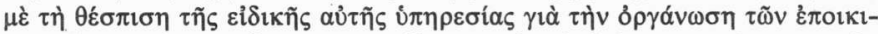

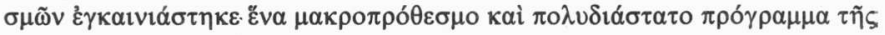

12. Angelov, ס. $\pi .$, б. 4.

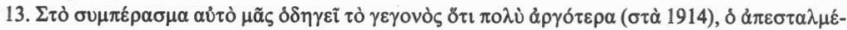

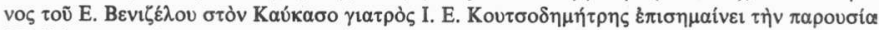

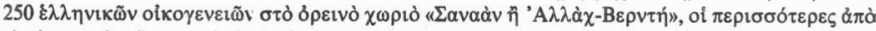

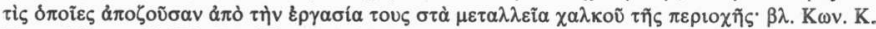

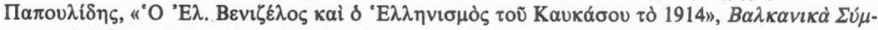

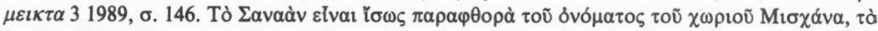

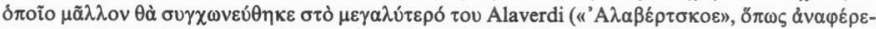

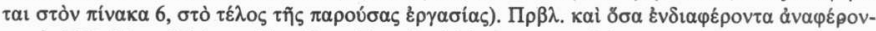

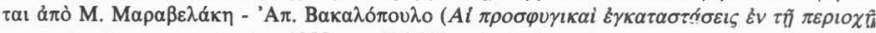

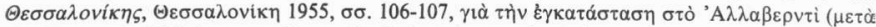

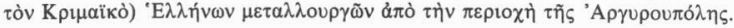

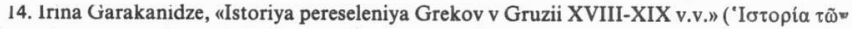

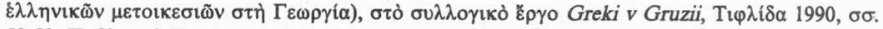

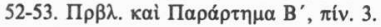




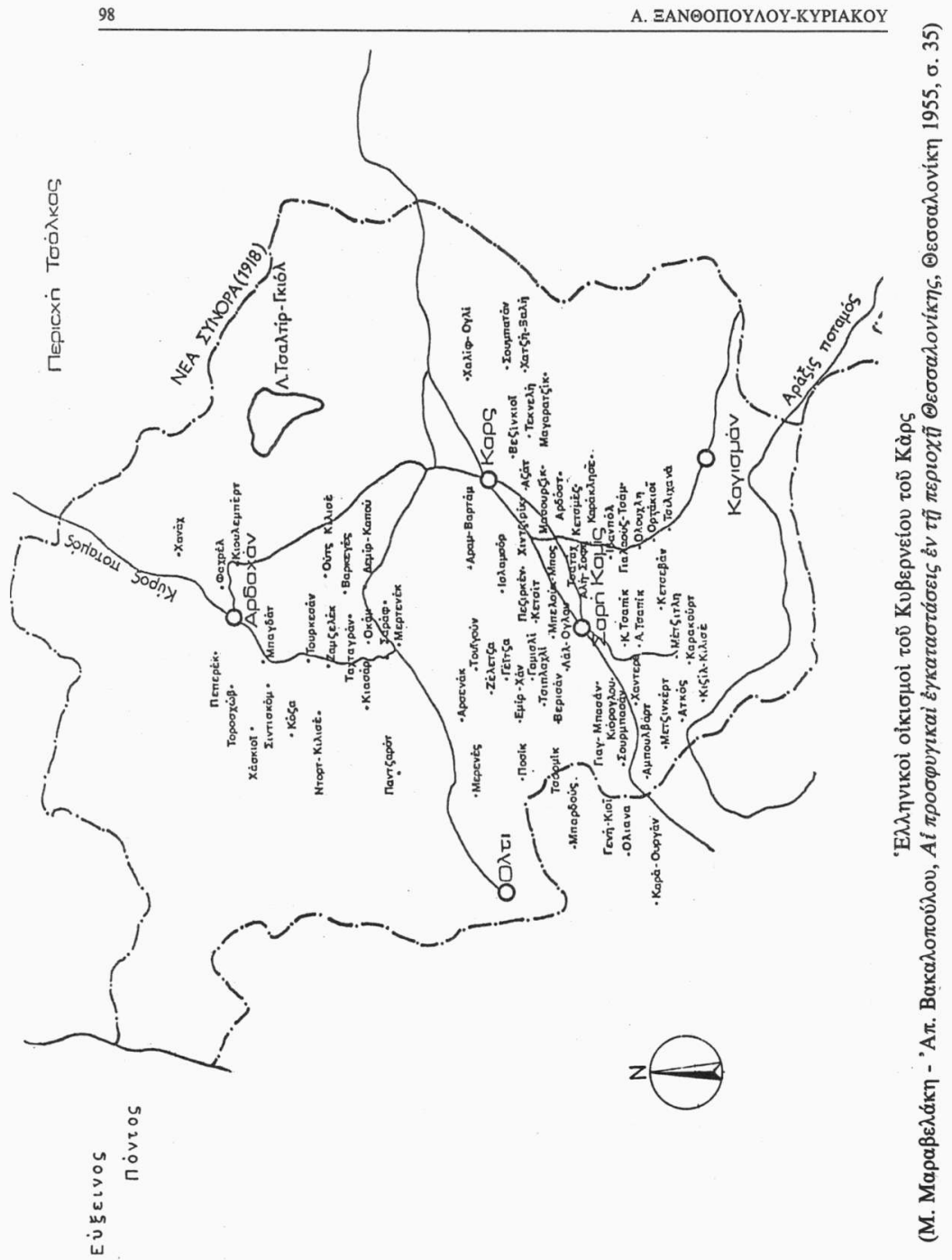




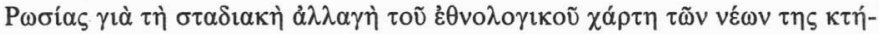

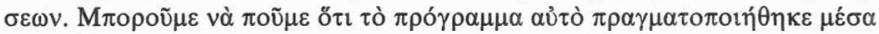

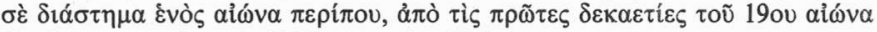

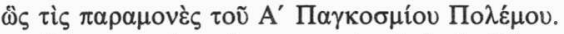

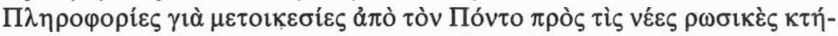

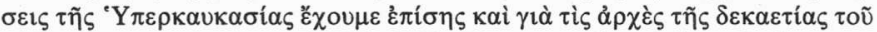

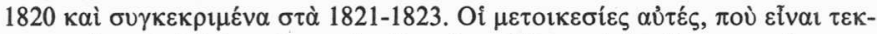

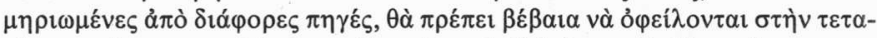

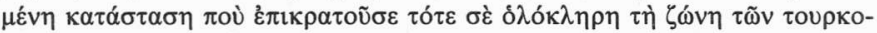

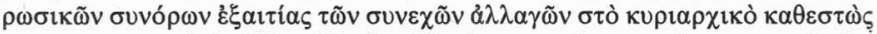

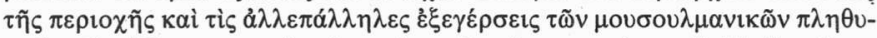

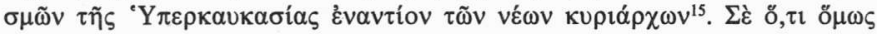

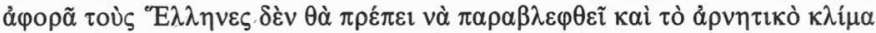

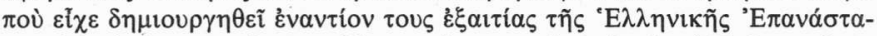

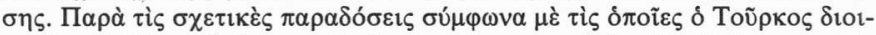

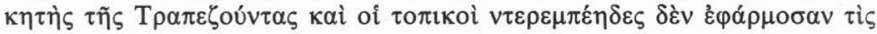

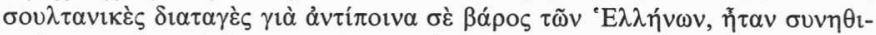

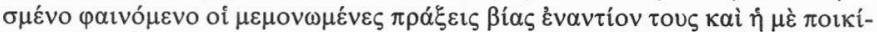

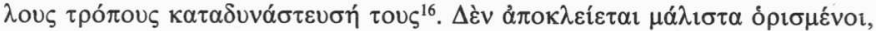

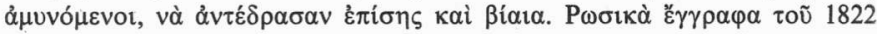

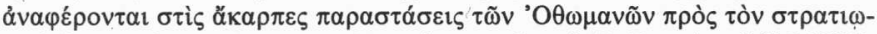

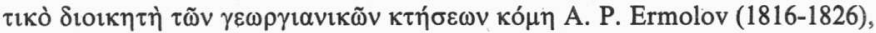

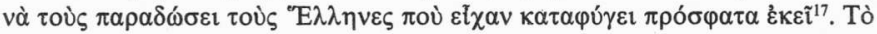

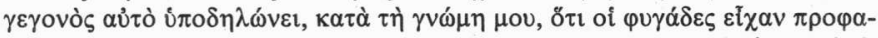

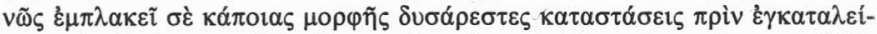

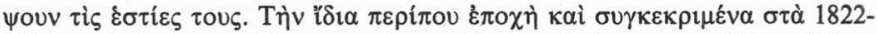

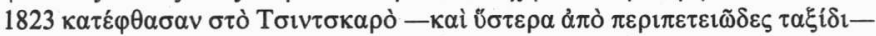

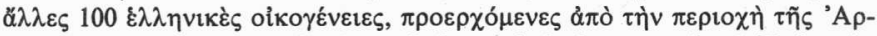

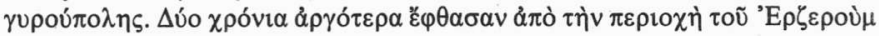

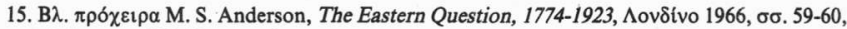

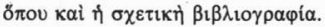

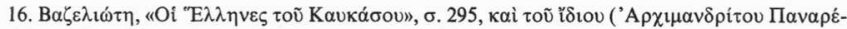

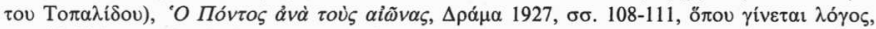

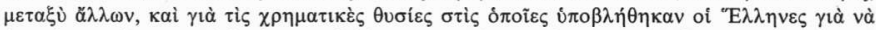

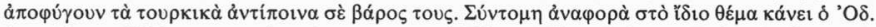

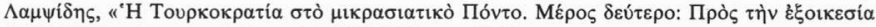

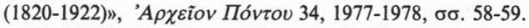

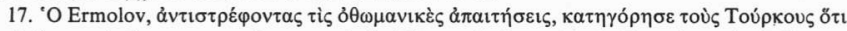

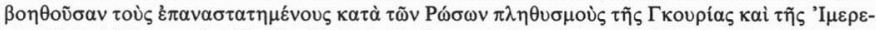

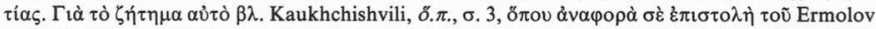

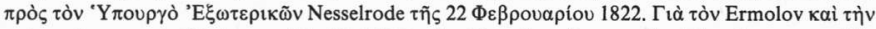

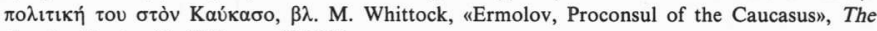
Russian Review 18, 1959, бo. 105-113. 


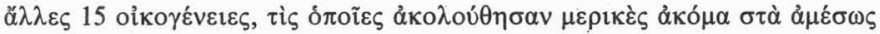

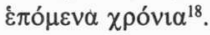

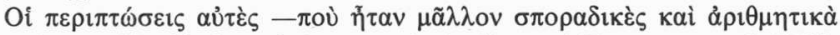

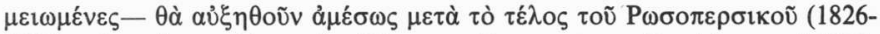

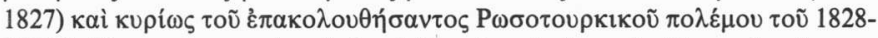

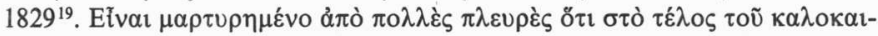

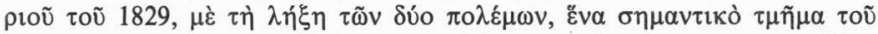

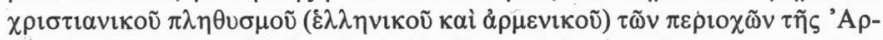

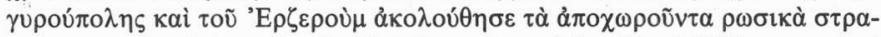

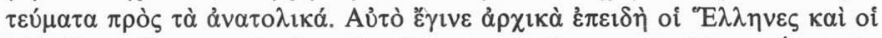

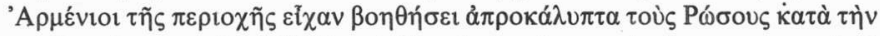

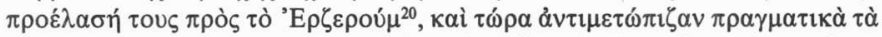

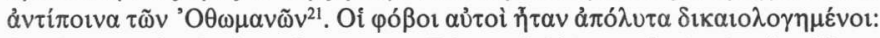

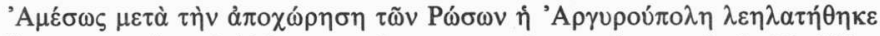

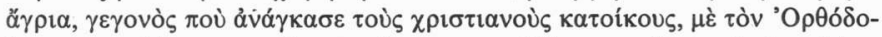

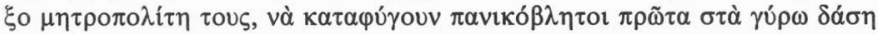

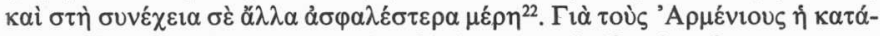

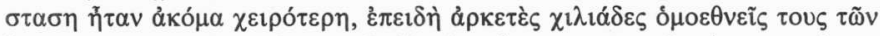
ঠ்

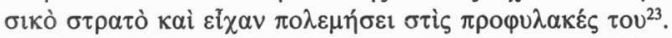

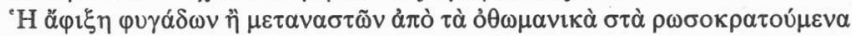

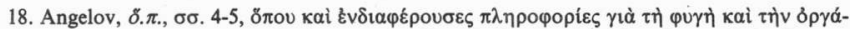

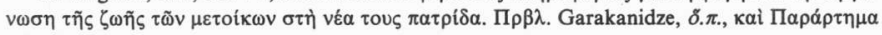
$\mathrm{B}^{\prime}, \pi$ ív. 3 .

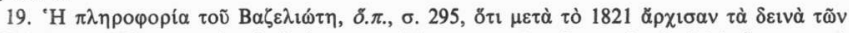

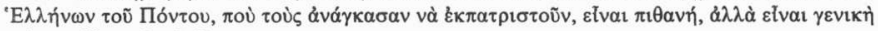

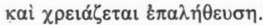

20. Felix Fonton, La Russie dans l'Asie Mineure ou campagne du Maréchal Paskevitch en 1828

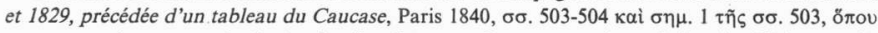

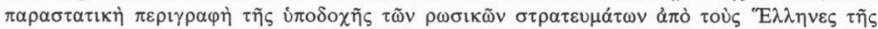

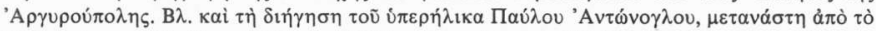

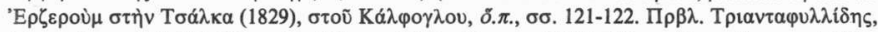

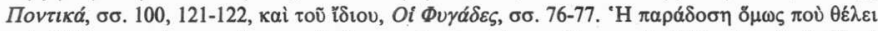

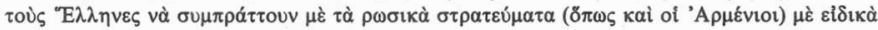

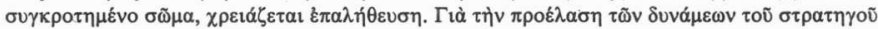

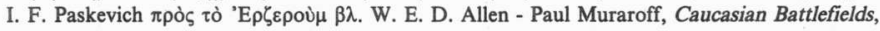

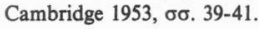

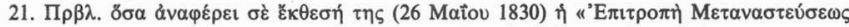

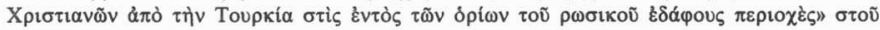
Kaukhchishvili, $\delta . \pi ., \sigma .4$.

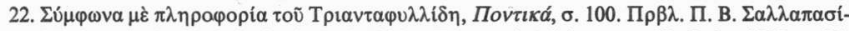

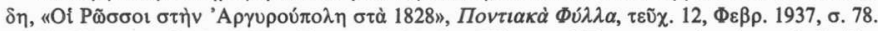

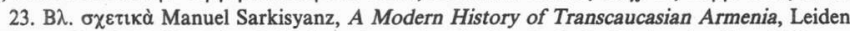

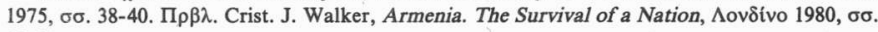
$52-54$. 


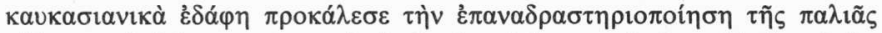

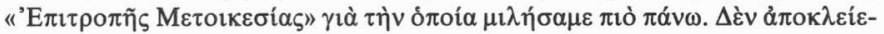

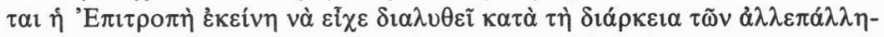

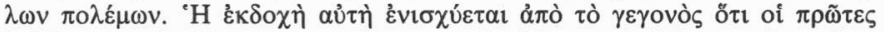

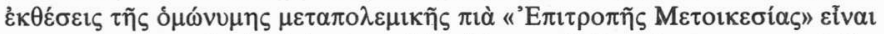

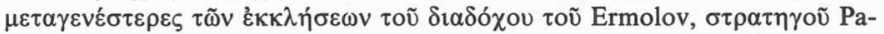

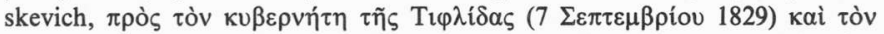

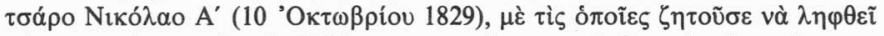

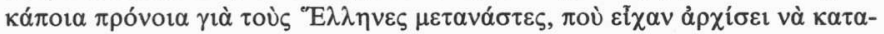

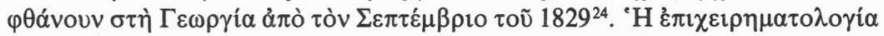

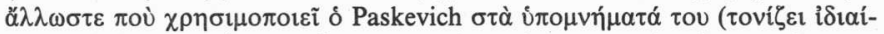

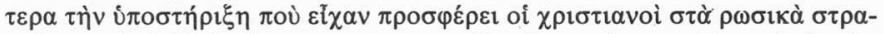

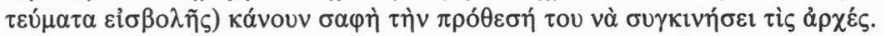

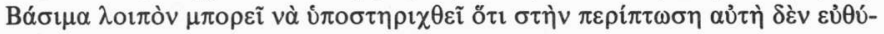

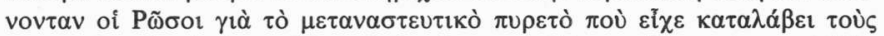

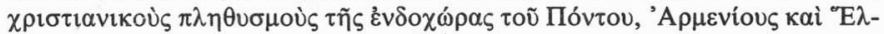

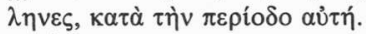

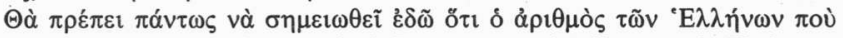

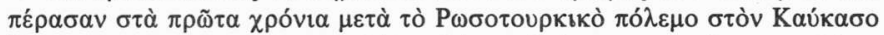

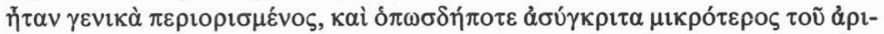

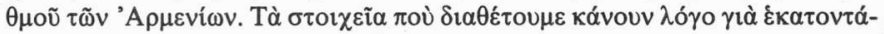

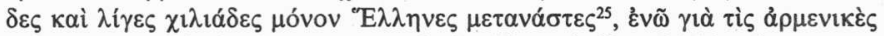

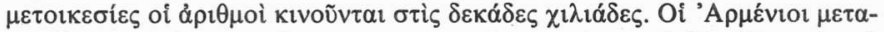

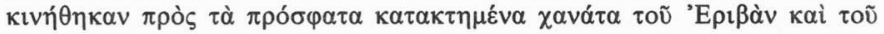

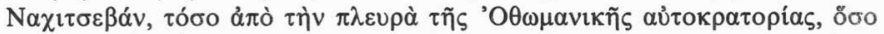

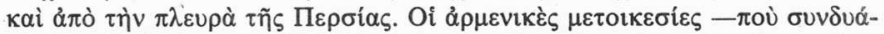

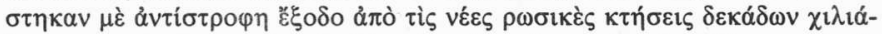

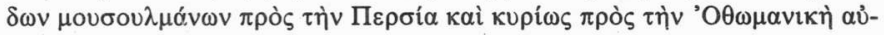

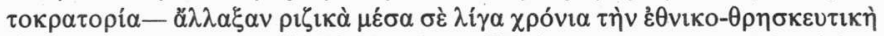

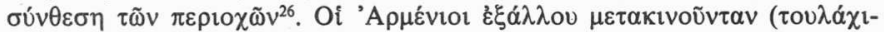

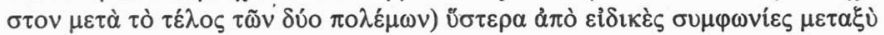

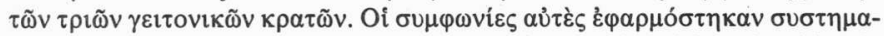

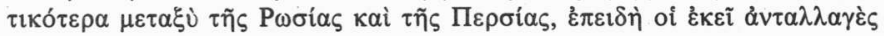

\section{Kaukhchishvili, $\delta . \pi .$, , .4.}

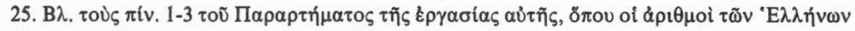

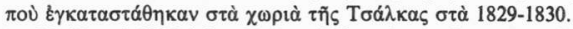

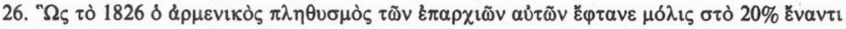

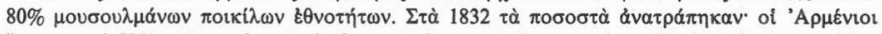

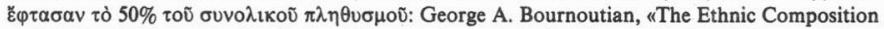
and the Socio-Economic Condition of Eastern Armenia in the First Half of the Nineteenth Cen-

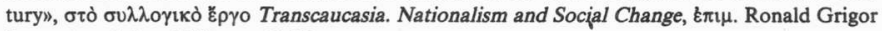
Suny, Ann Arbor 1983, oб. 69-86. 


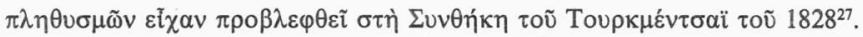

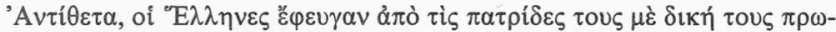

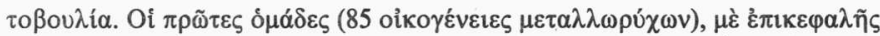

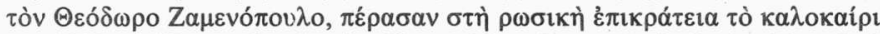

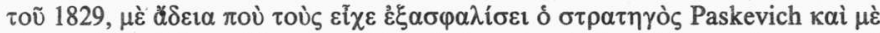

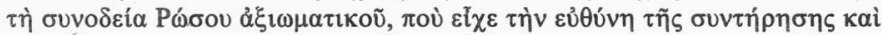

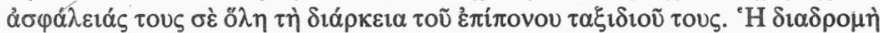

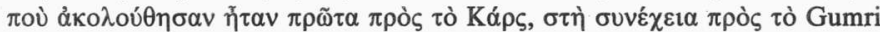

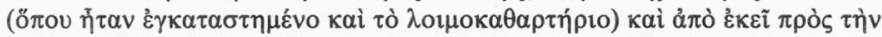

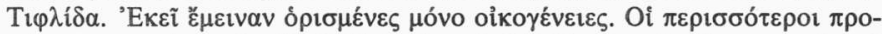

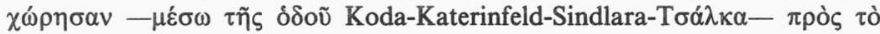

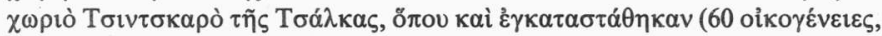

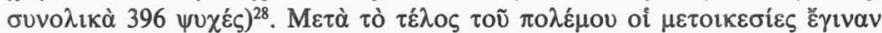

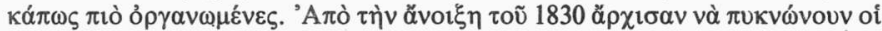

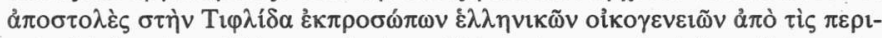

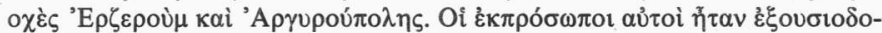

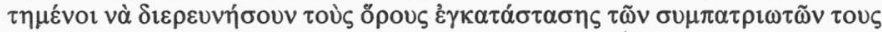

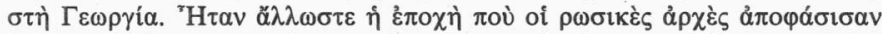

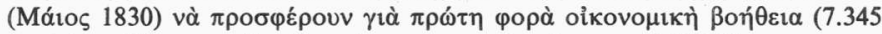

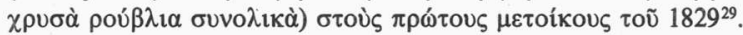

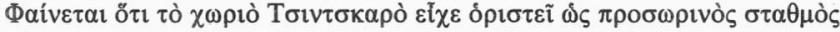

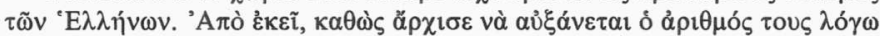

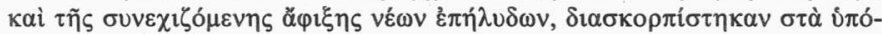

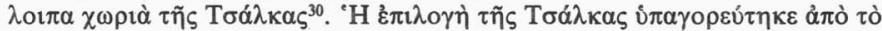

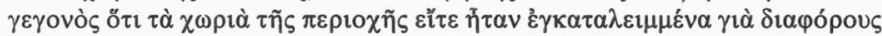

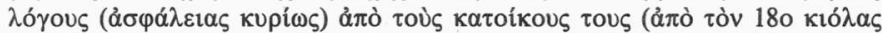

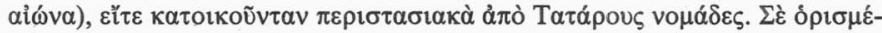

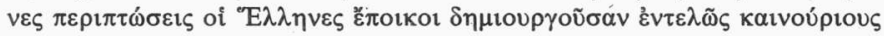

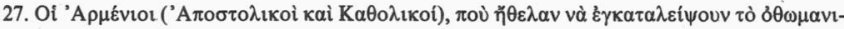

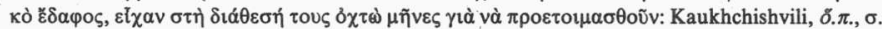

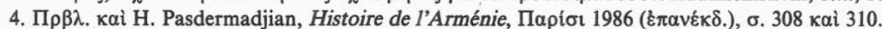

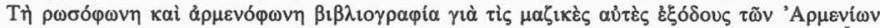

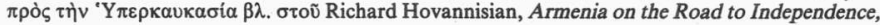

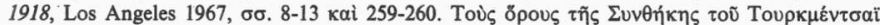

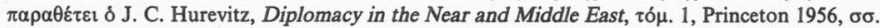
96-102.

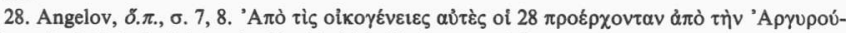

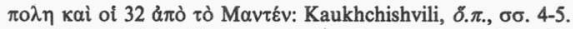

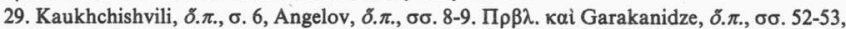

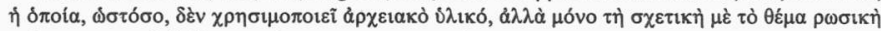

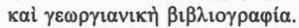

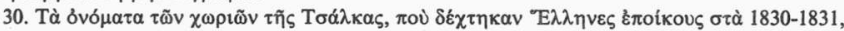

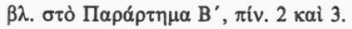




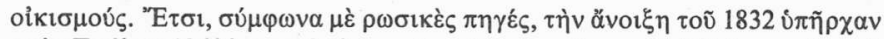

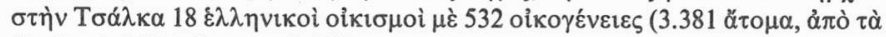

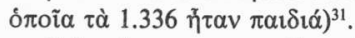

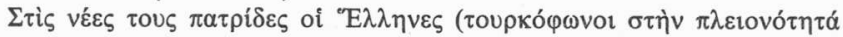

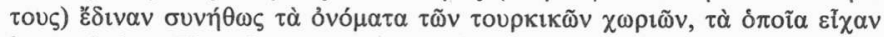

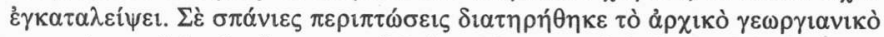

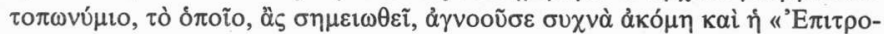

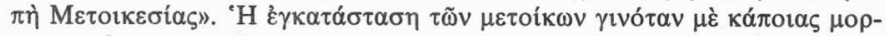

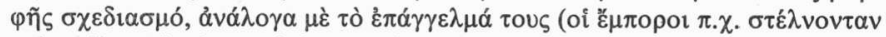

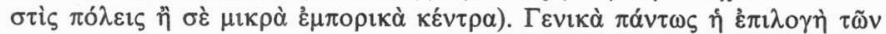

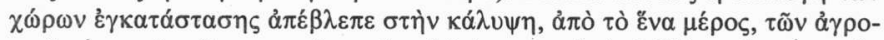

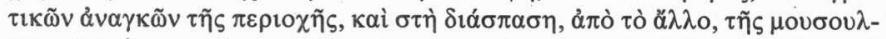

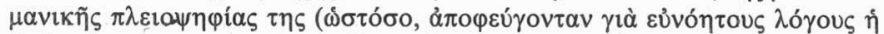

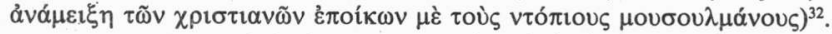

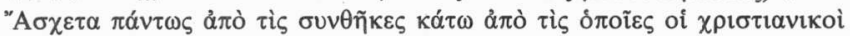

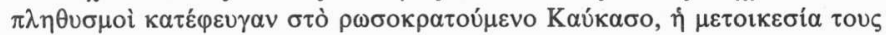

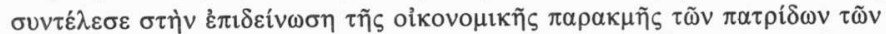

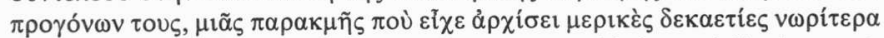

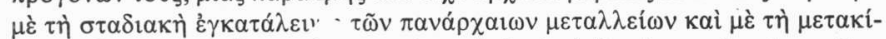

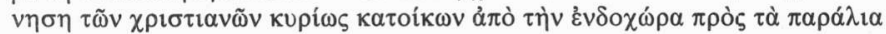

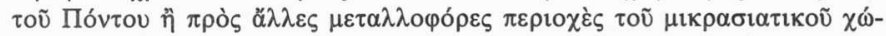

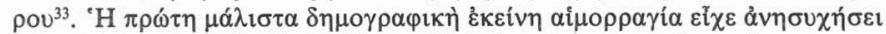

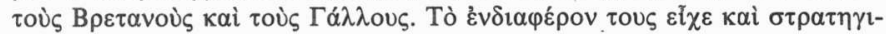

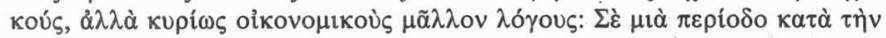

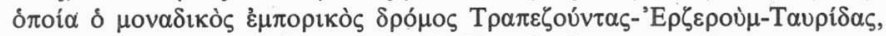

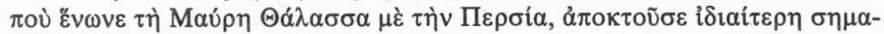

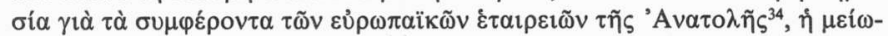

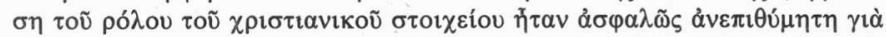

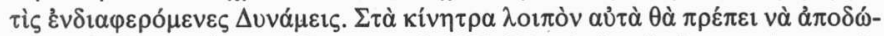

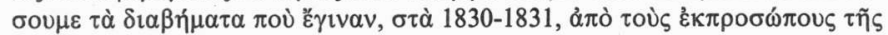

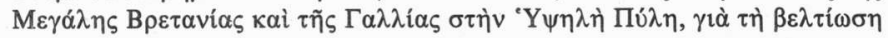

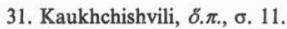

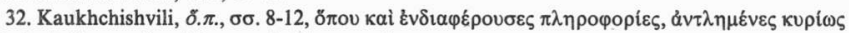

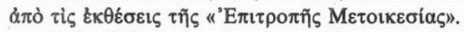

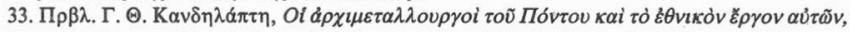

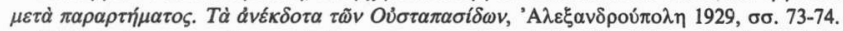

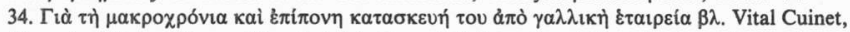
La Turquie d'Asie. Géographie administrative, statistique descriptive et raisonnée de chaque

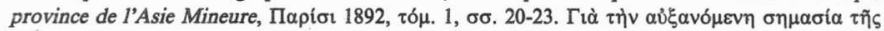

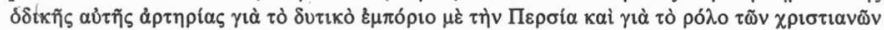

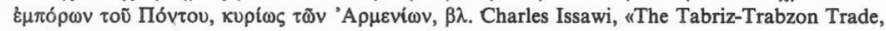
1830-1900: The Rise and Decline of a Route», International Journal of Middle East Studies 1, 1970, бо. 18-27. 


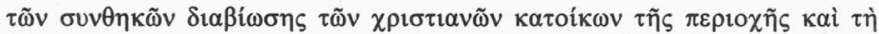

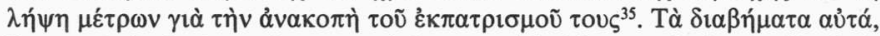

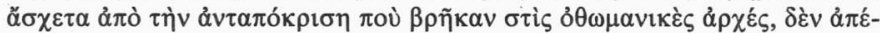

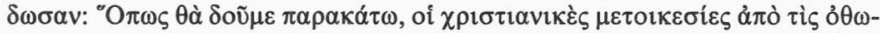

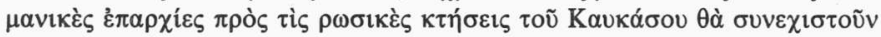

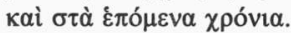

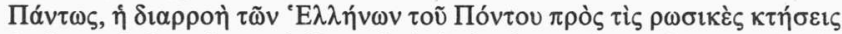

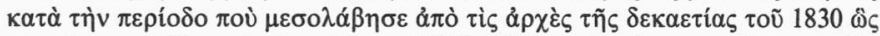

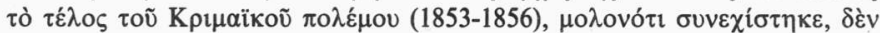

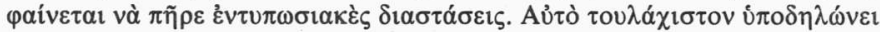
ex silentio 升 $\pi \lambda \hat{\eta} \tilde{\rho} \eta \varsigma$ है

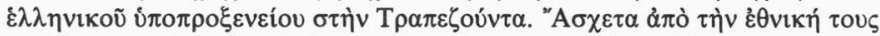

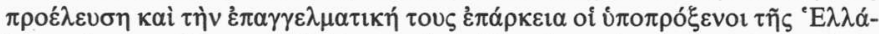

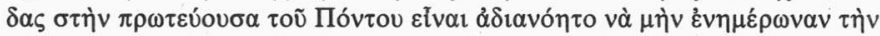

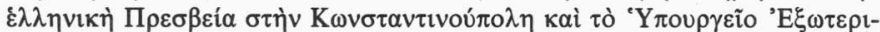

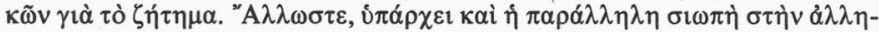

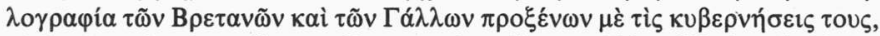

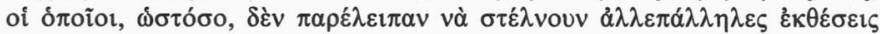

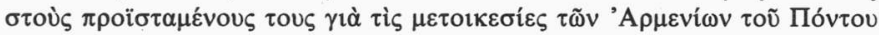

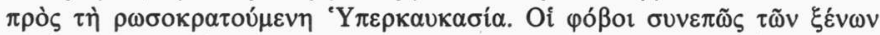

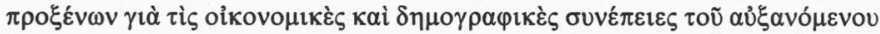

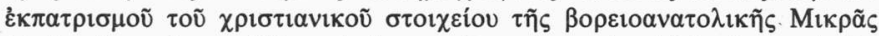

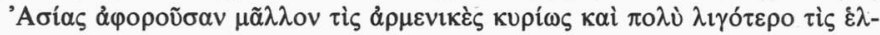

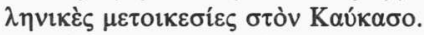

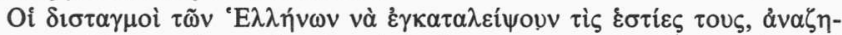

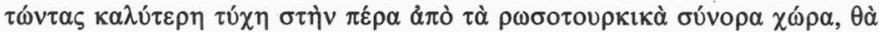

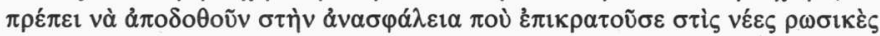

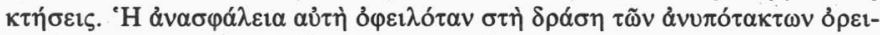

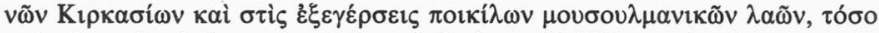

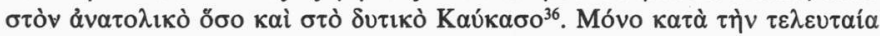

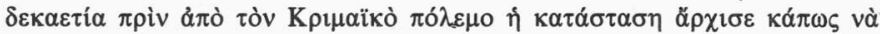

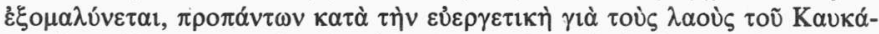

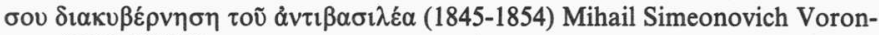
tsov $(1782-1856)^{37}$.

35. Archives du Ministère des Affaires Étrangères, Correspondance Politique Consulaire ( $\sigma \tau \grave{\partial}$

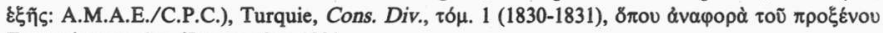

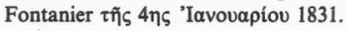

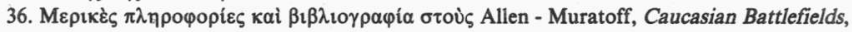
бб. 48-53.

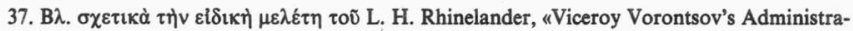

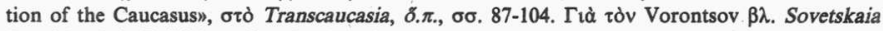

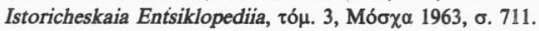




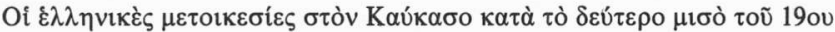

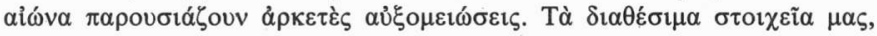

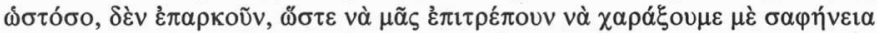

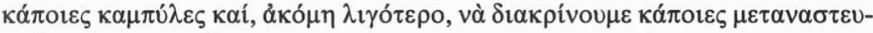

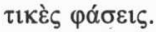

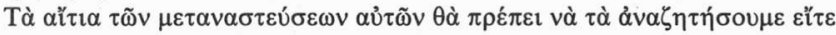

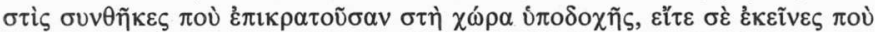

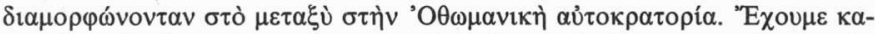

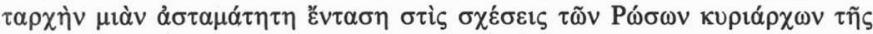

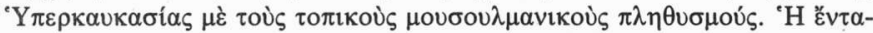

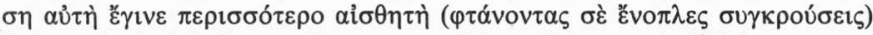

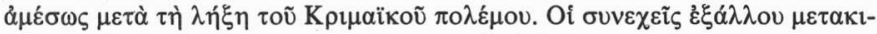

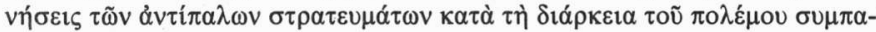

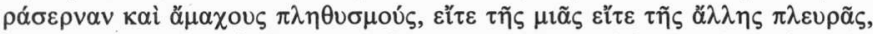

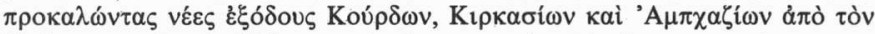

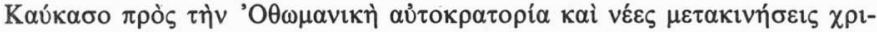

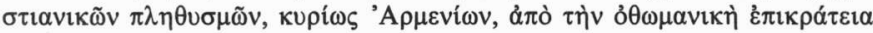

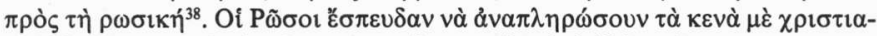

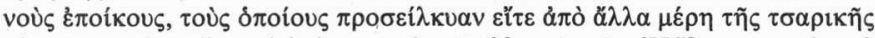

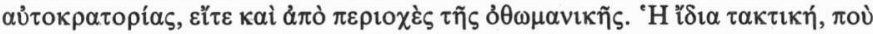

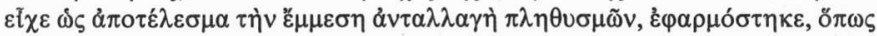

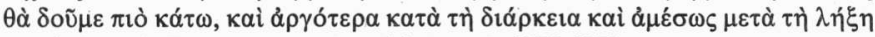

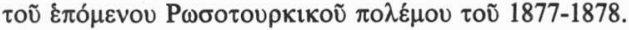

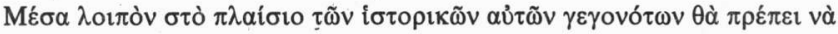

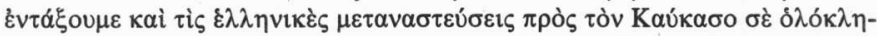

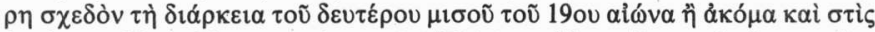

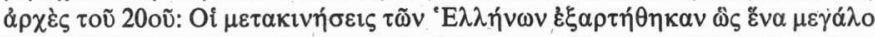

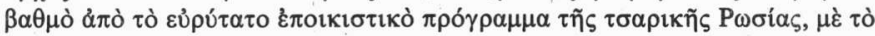

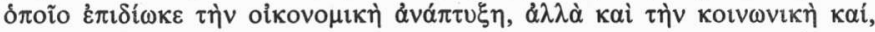

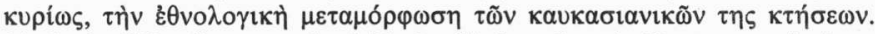

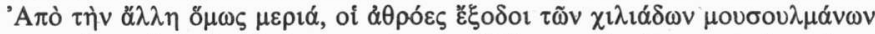

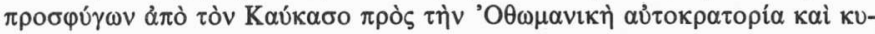

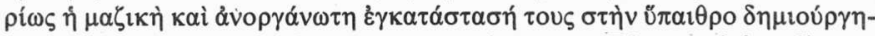

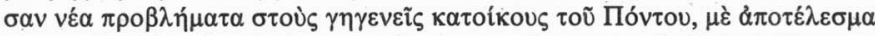

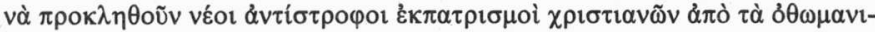

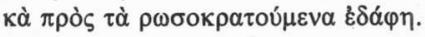

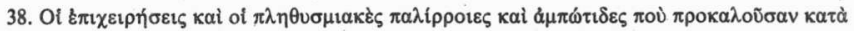

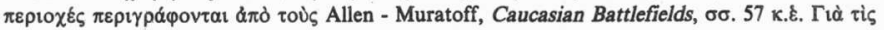

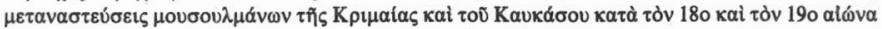

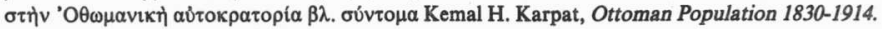
Demographic and Social Characteristics, Wisconsin 1985, oб. 65-70. 


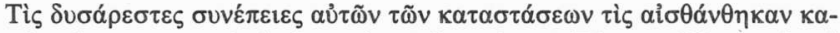

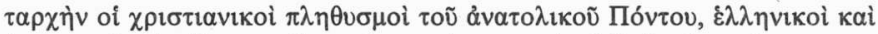

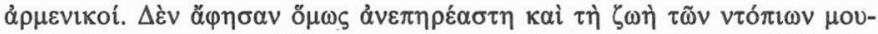

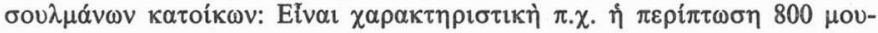

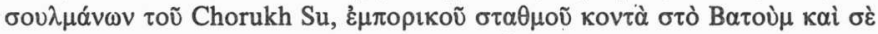

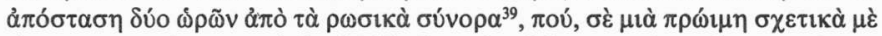

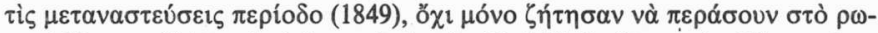

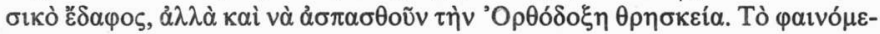

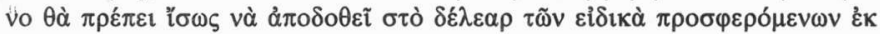

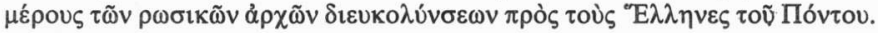

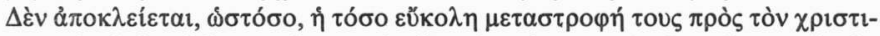

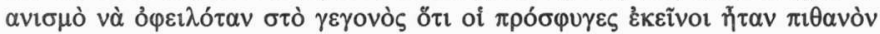

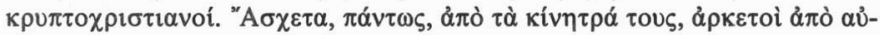

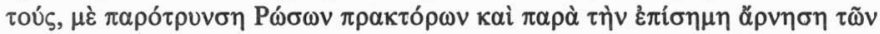

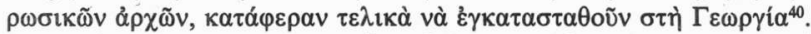

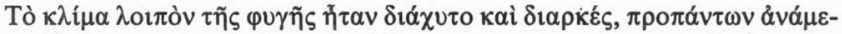

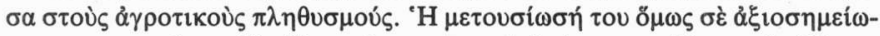

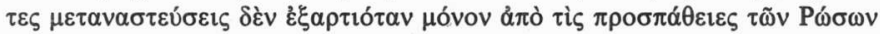

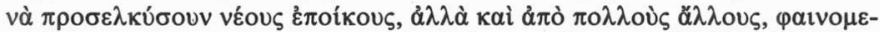

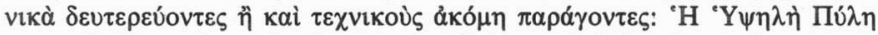

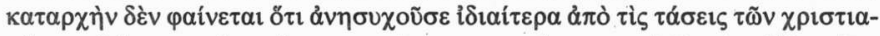

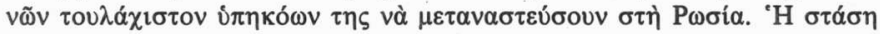

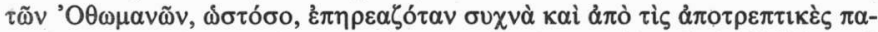

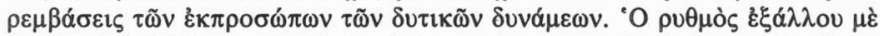

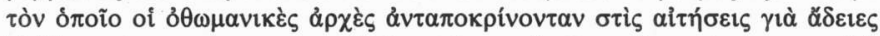

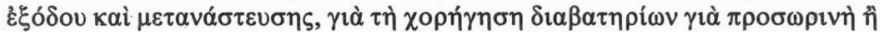

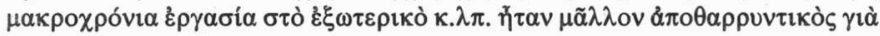

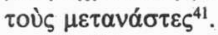

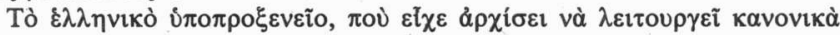

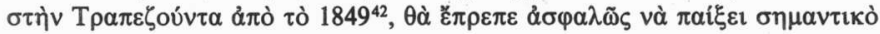

39. James Brant, «Journey Through a Part of Armenia and Asia Minor in the Year 1835», The

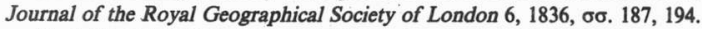

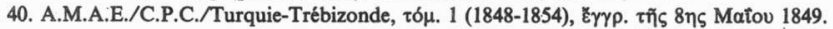

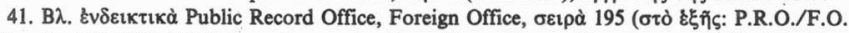

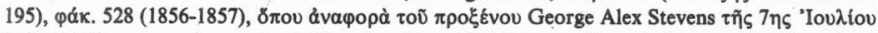

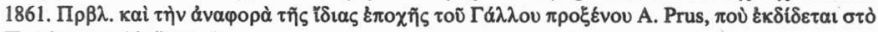

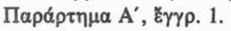

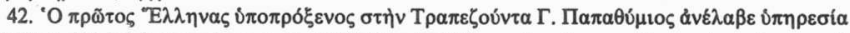

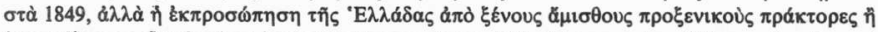

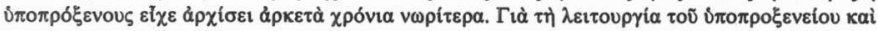

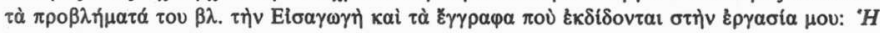

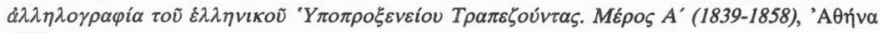
1995. 


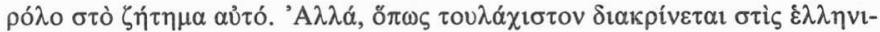

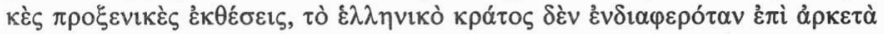

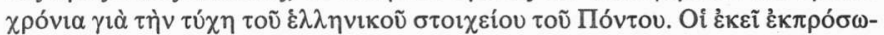

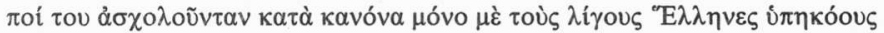

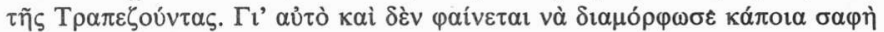

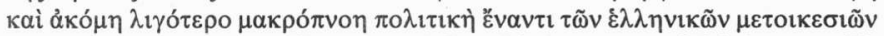

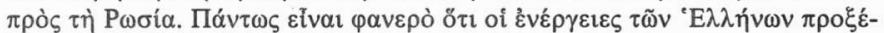

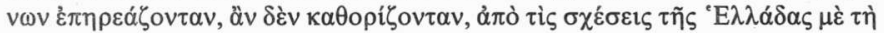

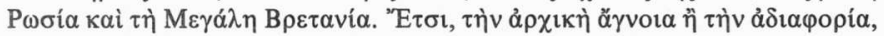

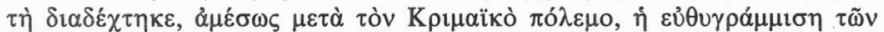

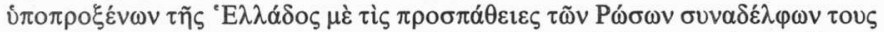

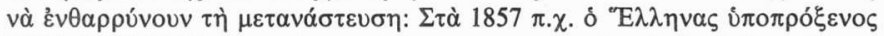

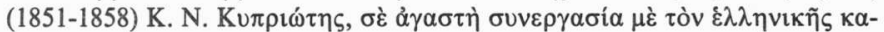

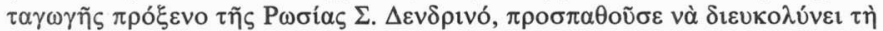

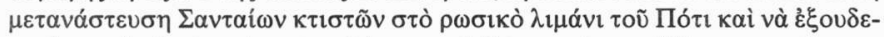

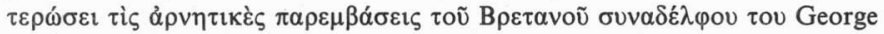

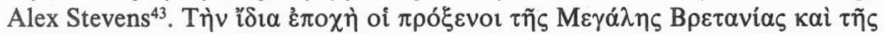

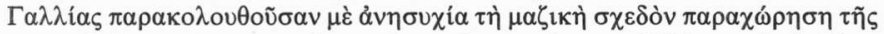
$\rho \omega \sigma เ \kappa \tilde{\varsigma}$

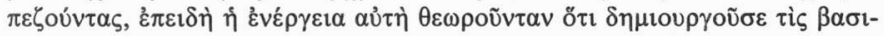

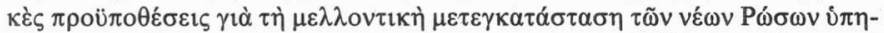

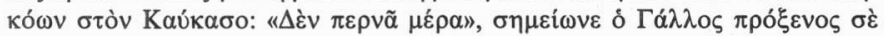

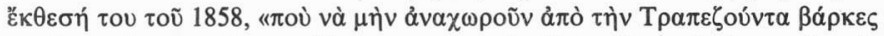

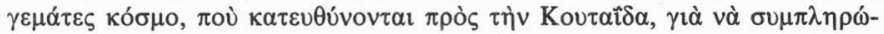

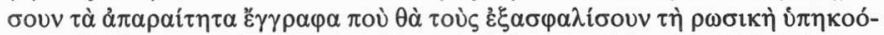

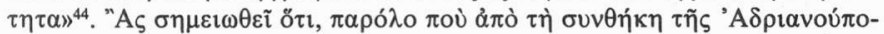

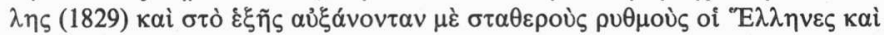

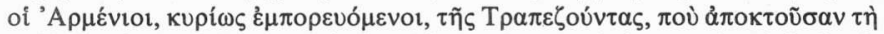

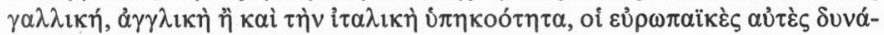

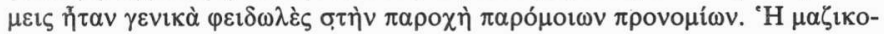

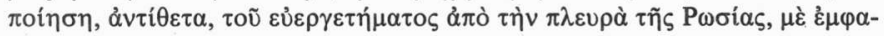

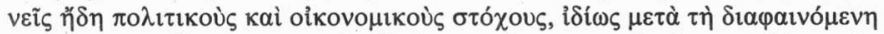

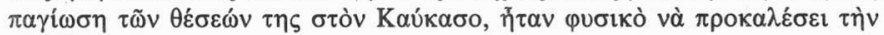

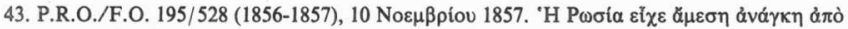

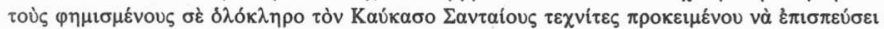

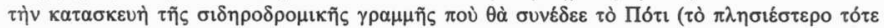

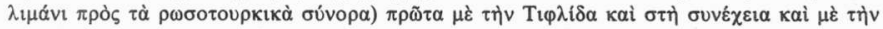

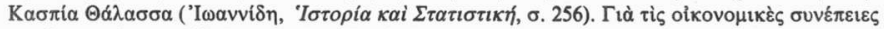

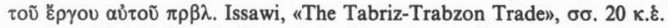

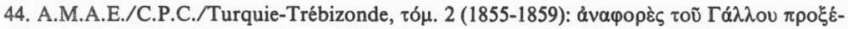

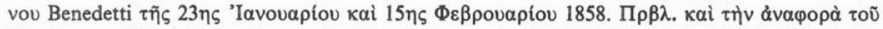

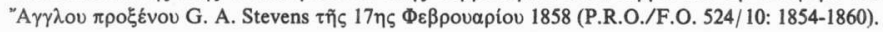




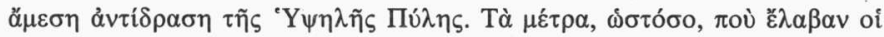

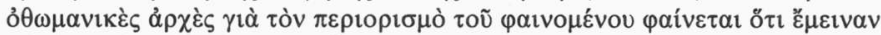

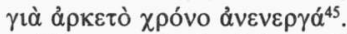

'H

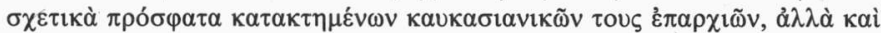

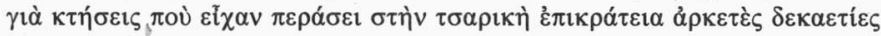

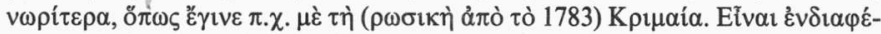

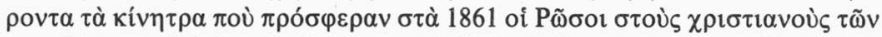

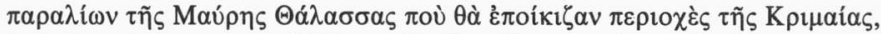

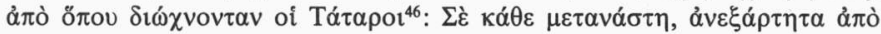

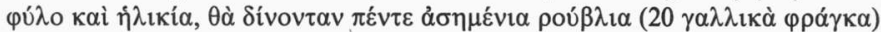

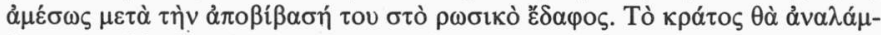

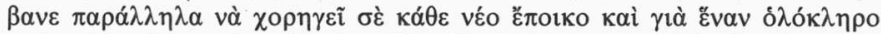

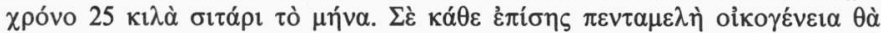

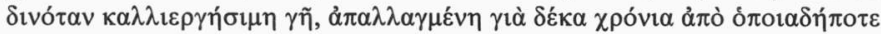

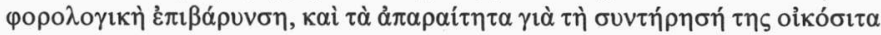

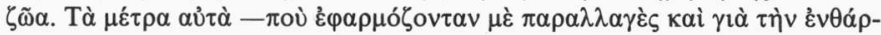

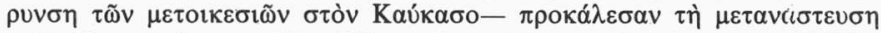

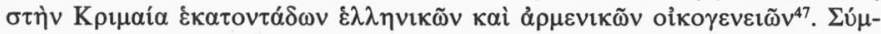

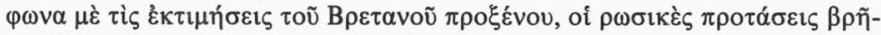

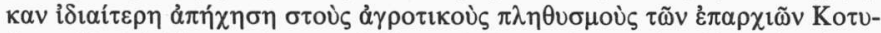

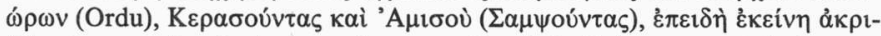

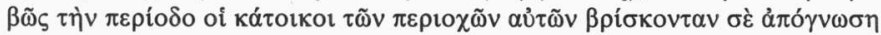

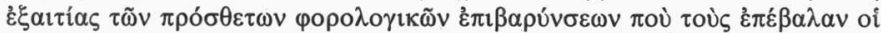

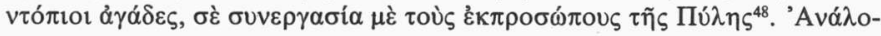

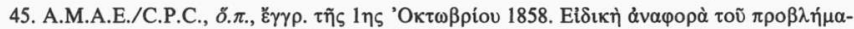
$\tau \circ \varsigma$ $\sigma \tau$ oṽ Üner Turgay, «Trade and Merchants in Nineteenth-Century Trabzon: Elements of Ethnic

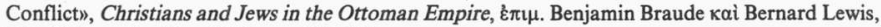

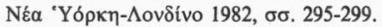

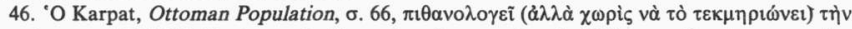

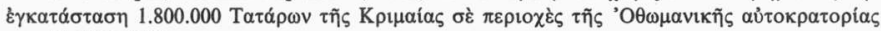
$\mu \varepsilon \tau \alpha \xi \grave{~} 1783-1922$.

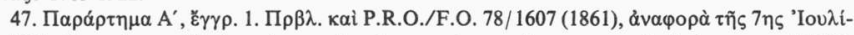

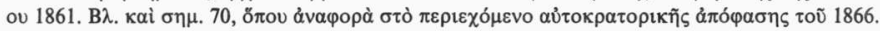

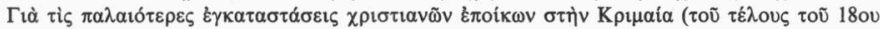

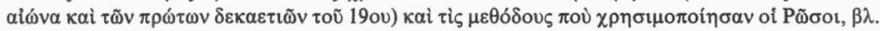
V. Karidis, "The Mariupol Greeks: Tsarist Treatment of an Ethnic Minority ca. 1778-1859", Journal of Modern Hellenism 3, 1986, бб. 57-74.

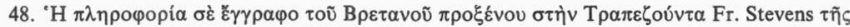

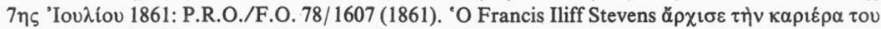

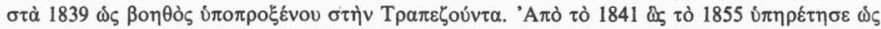

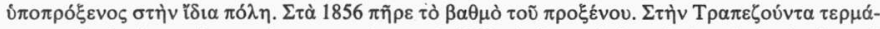

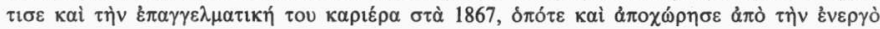




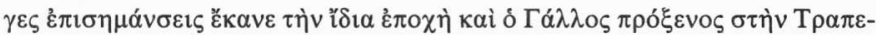

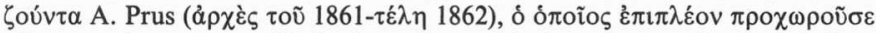

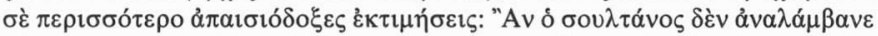

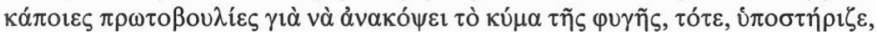

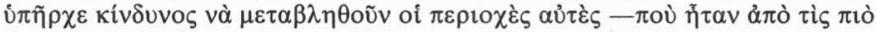

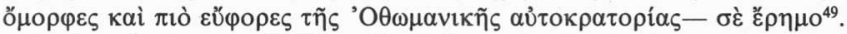

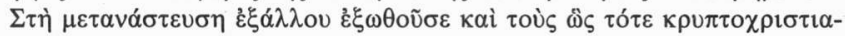

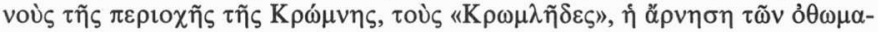

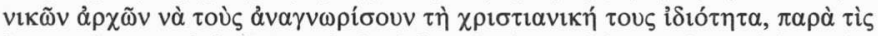

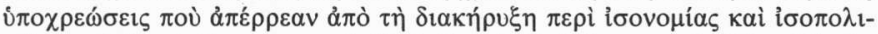

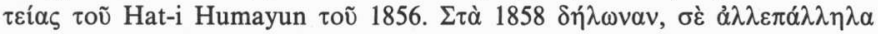
نં

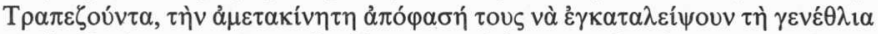

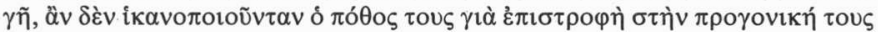

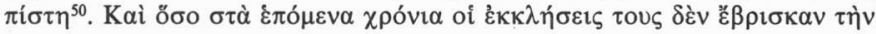

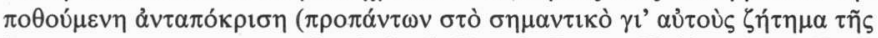

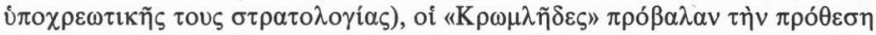

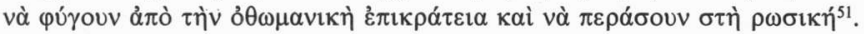

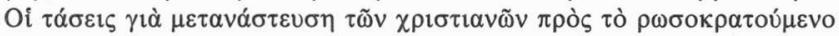

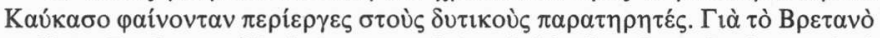

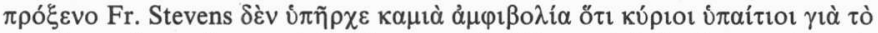

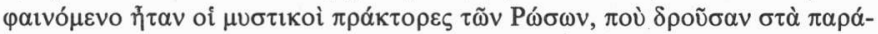

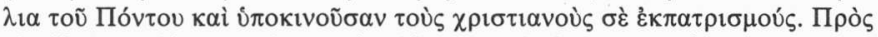

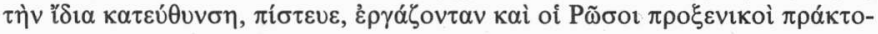

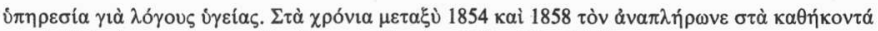

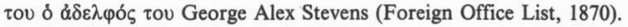

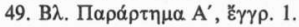

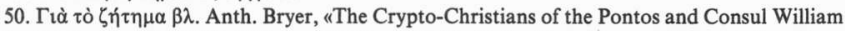

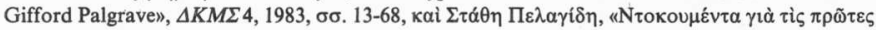

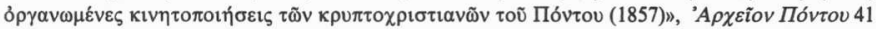

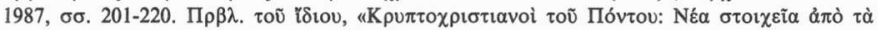

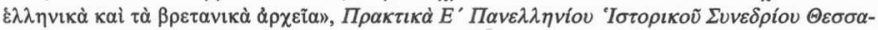

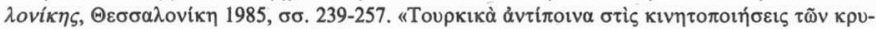

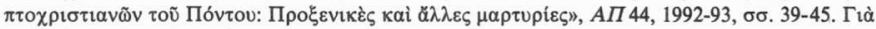

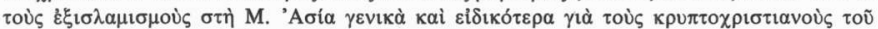

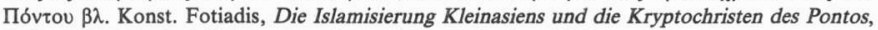

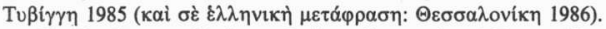

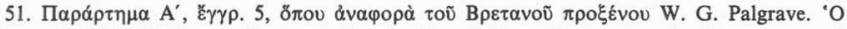

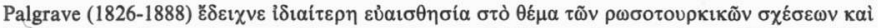

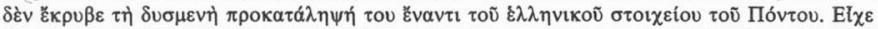

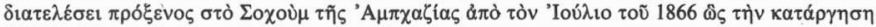

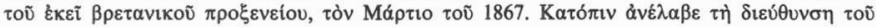

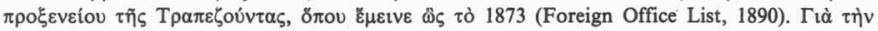

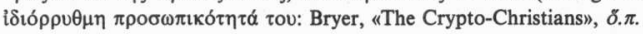




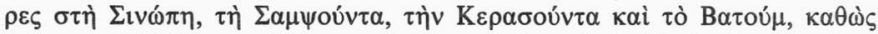

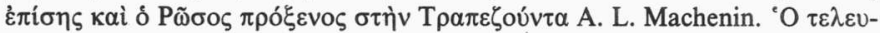

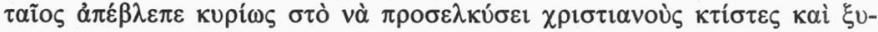

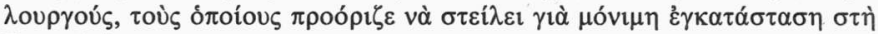
$\Gamma \varepsilon \omega \rho \gamma i \alpha^{52}$.

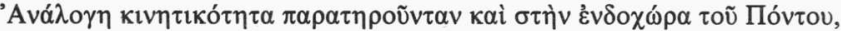

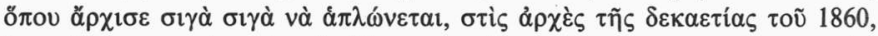

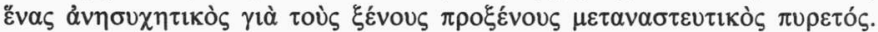

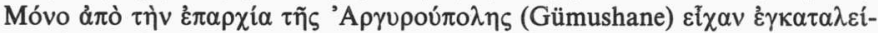

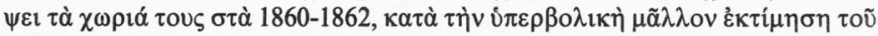

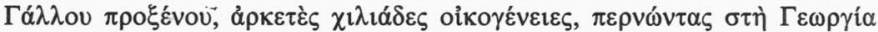

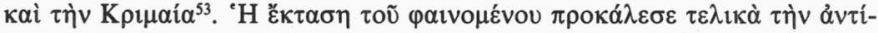

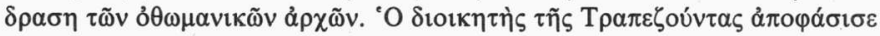

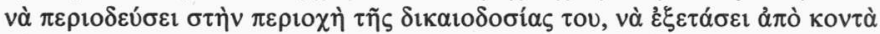

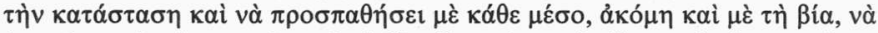

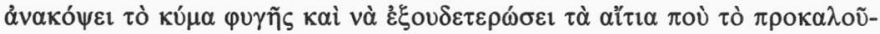

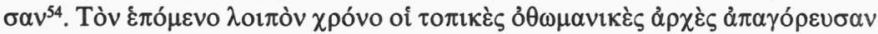

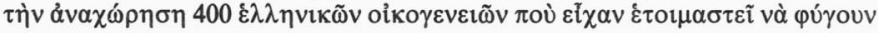

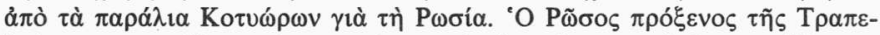

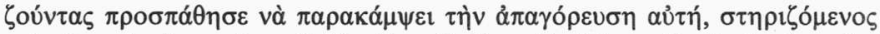

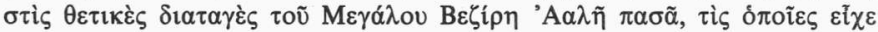

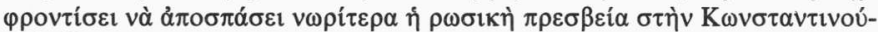
$\pi \circ \lambda \eta^{55}$.

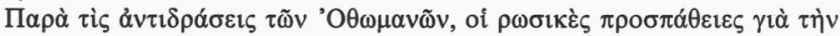

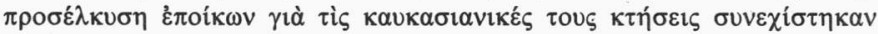

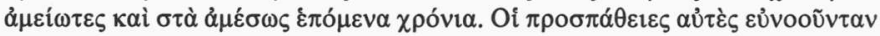

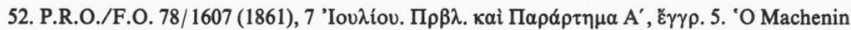

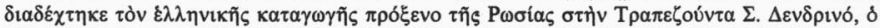

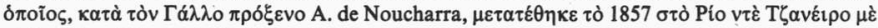

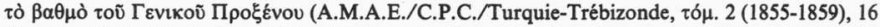

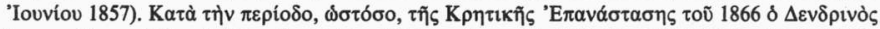

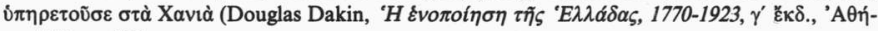
$v \alpha 1989, \sigma .170)$.

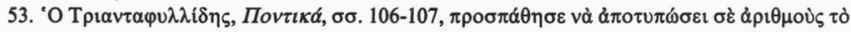

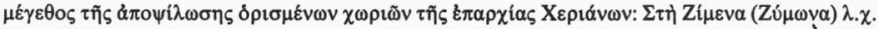

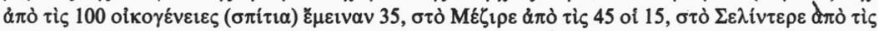

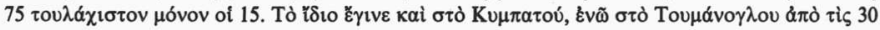

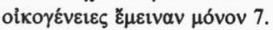

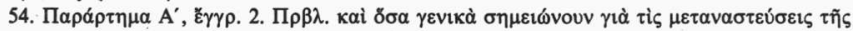

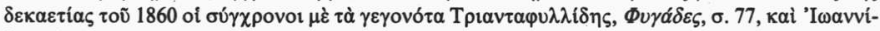

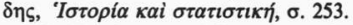

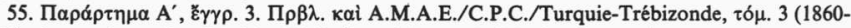

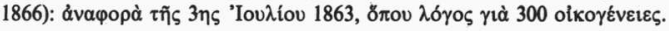




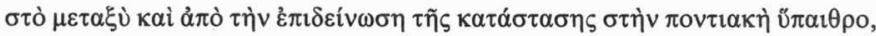

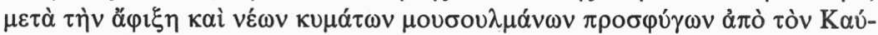

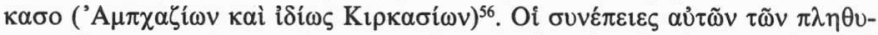

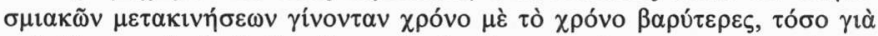

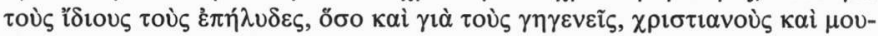

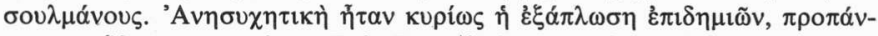

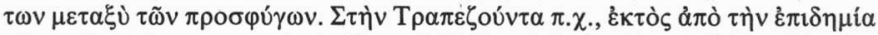

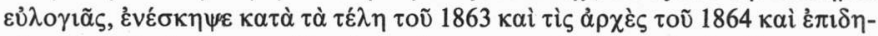

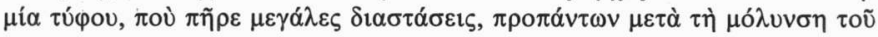

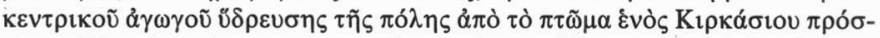

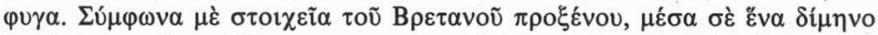

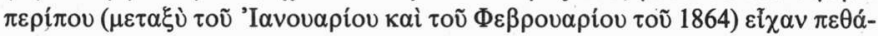

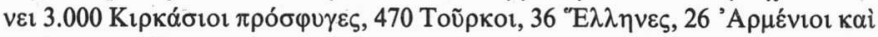

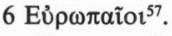

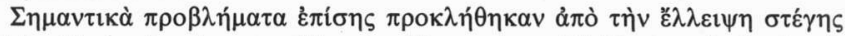

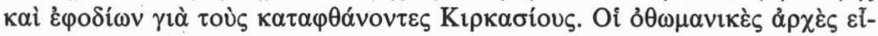

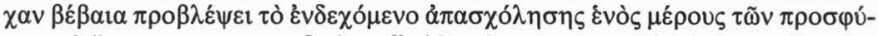

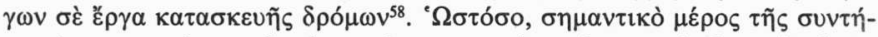

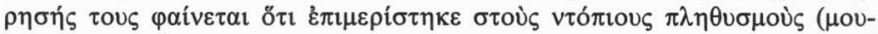

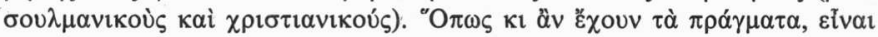

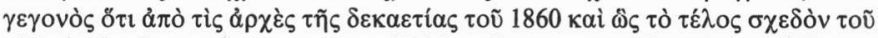

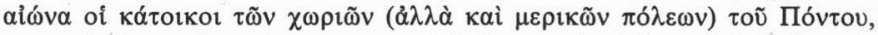

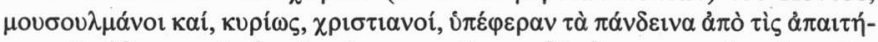

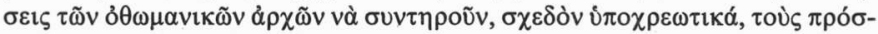

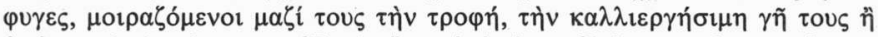

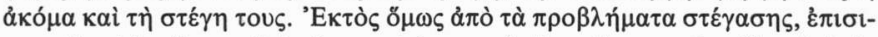

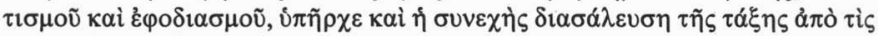

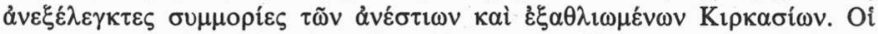

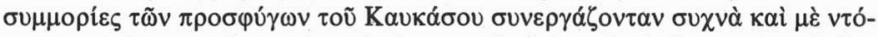

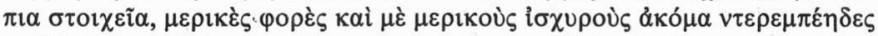

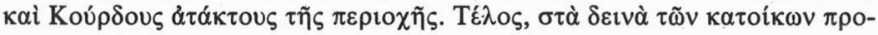

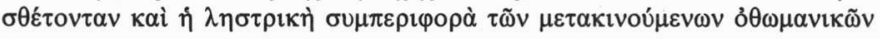

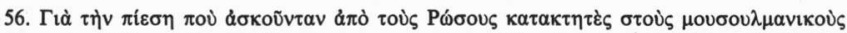

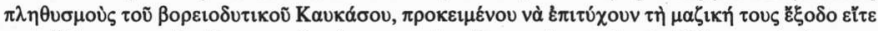

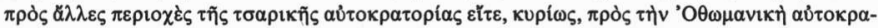
$\tau \rho \rho i \alpha, \beta \lambda$. $\pi \rho \delta ́ \chi \varepsilon i \rho \alpha$ Kazemzadeh, «Russian Penetration of the Caucasus», $\sigma \sigma$. 247-263.

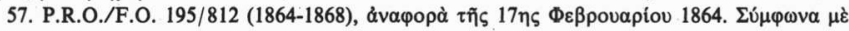

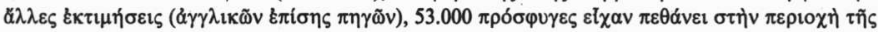

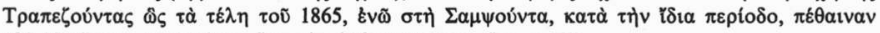

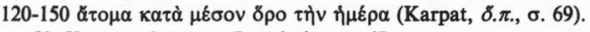

58. Karpat, Ottoman Population, б. 67. 


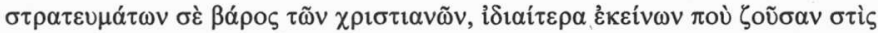
$\pi \alpha \rho \alpha \mu \varepsilon \theta$ ó $\rho є \varsigma, \pi \varepsilon \rho \imath \chi \chi \varepsilon \varsigma_{\zeta}^{59}$.

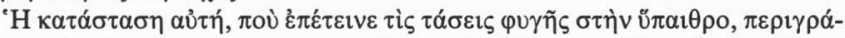

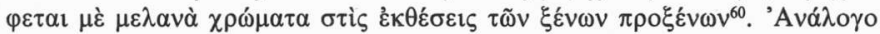

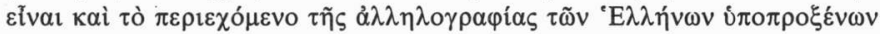

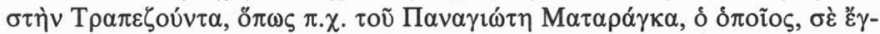

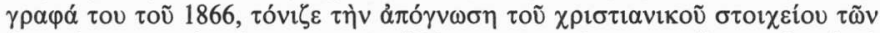

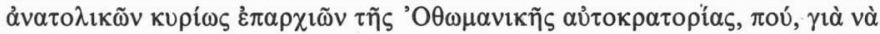

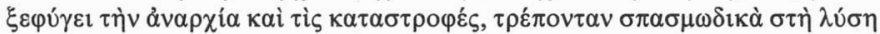

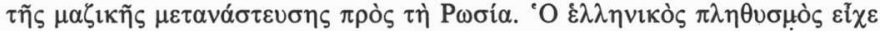

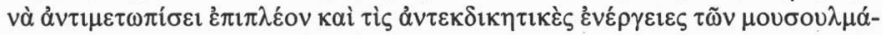

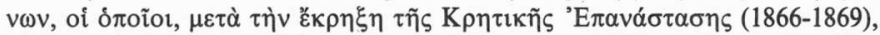

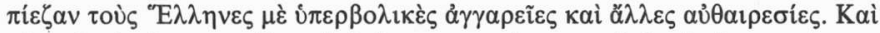

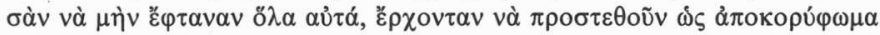

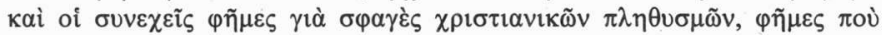

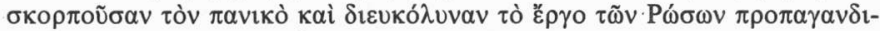
$\sigma \tau \tilde{\omega} v \tau \tilde{\eta} \varsigma \mu \varepsilon \tau \alpha v \alpha ́ \sigma \tau \varepsilon v \sigma \eta \varsigma^{61}$.

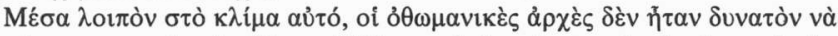

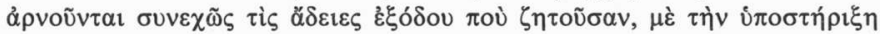

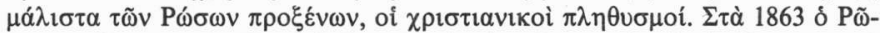

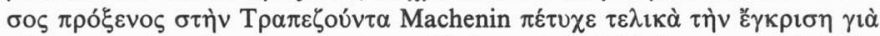

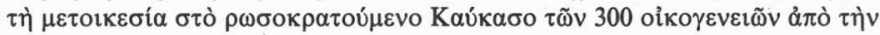

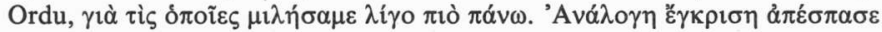

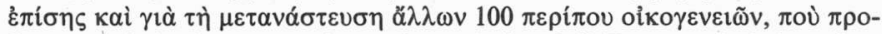

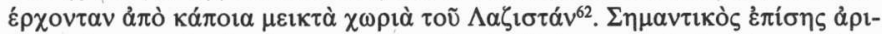

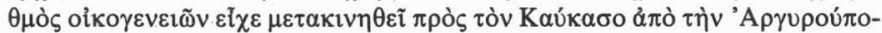

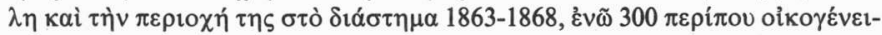

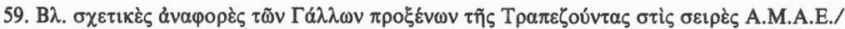

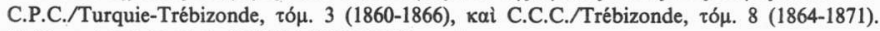

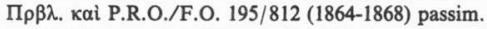

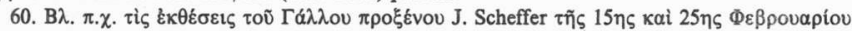

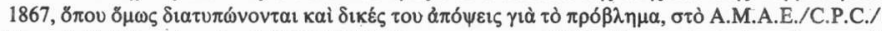

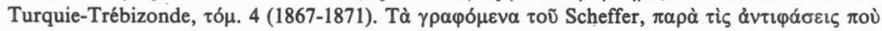

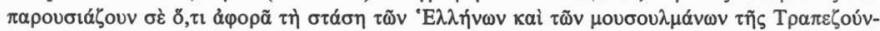

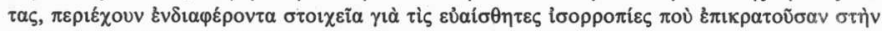

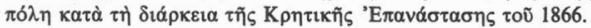

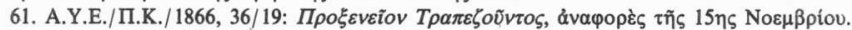

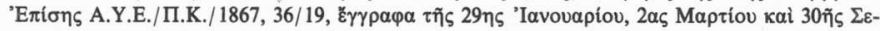

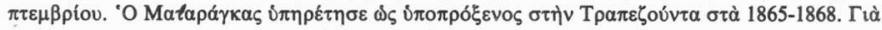

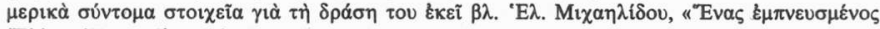
"Е $\lambda \lambda \eta \nu$ 'Ү 65-66.

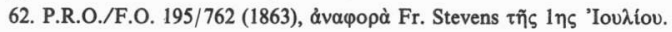




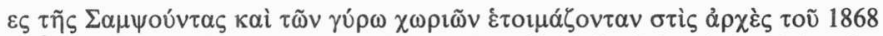

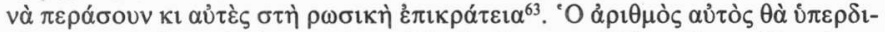

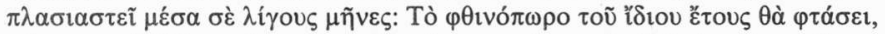

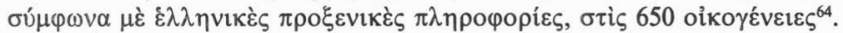

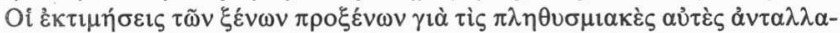

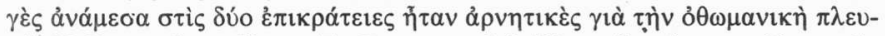

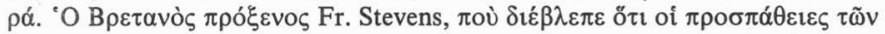

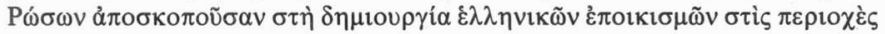

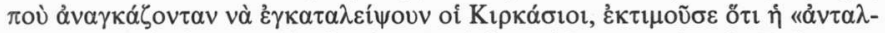

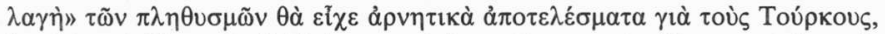

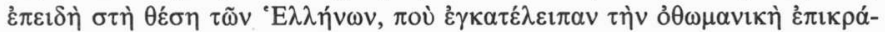

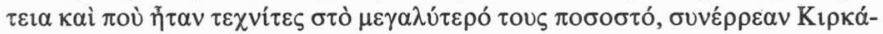
$\sigma ı$, «totally unfit for work of any kind» ${ }^{65}$. 'Avói

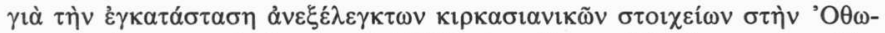

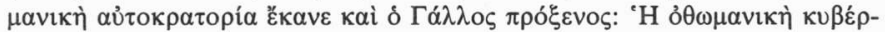

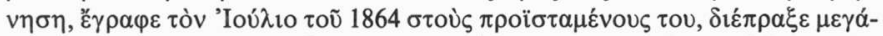

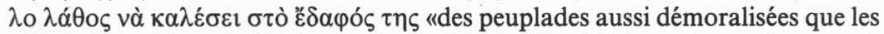

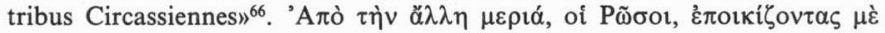

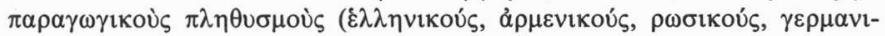

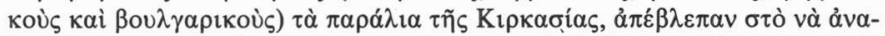

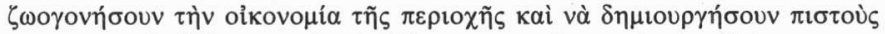

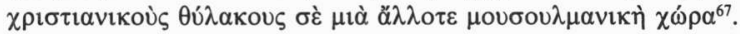

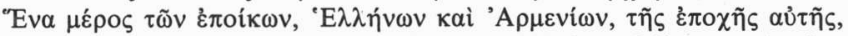

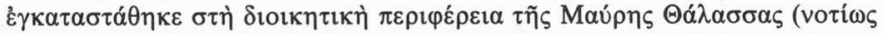

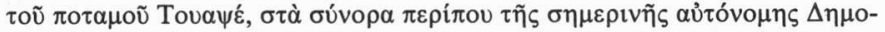

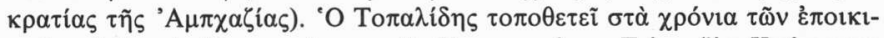

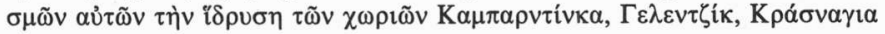

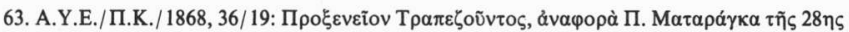

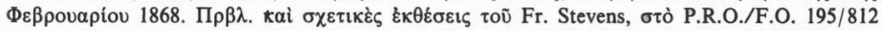

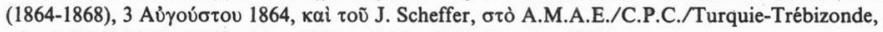

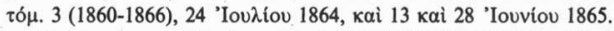

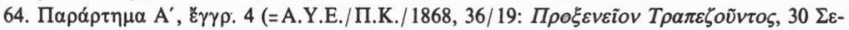

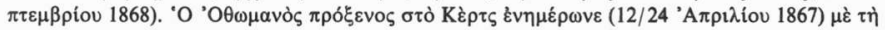

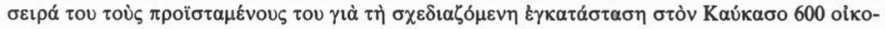

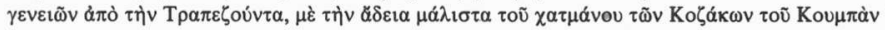

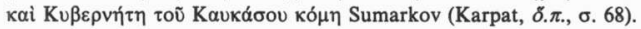

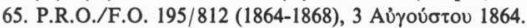

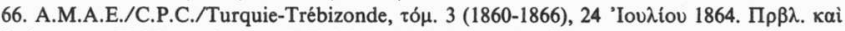

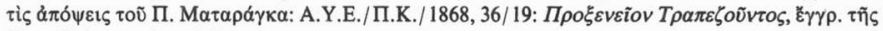

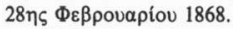

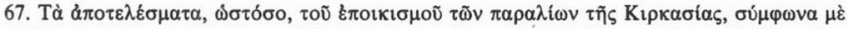

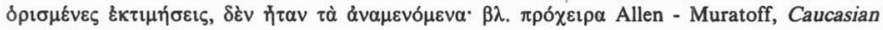
Battlefields, $\sigma .108$. 


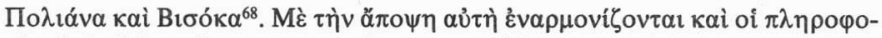

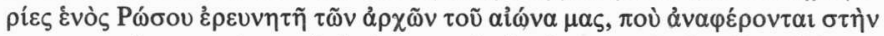

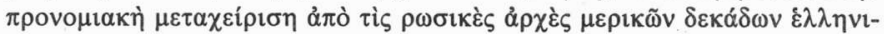

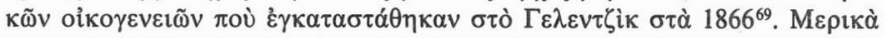

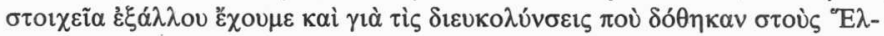

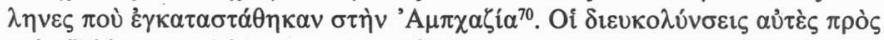

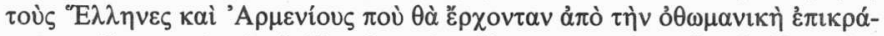

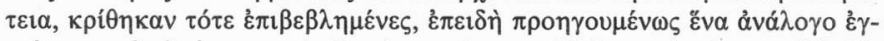

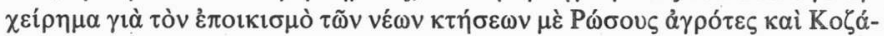

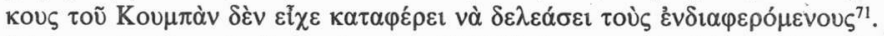

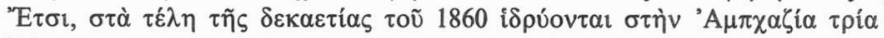

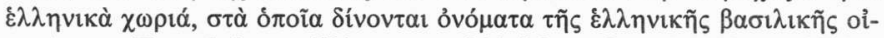

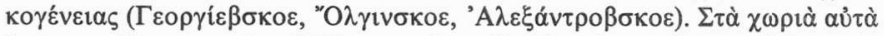

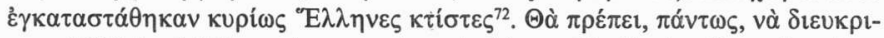

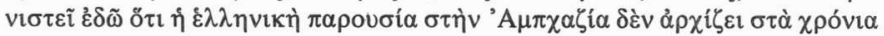
$\alpha$ น̉น́. 'A

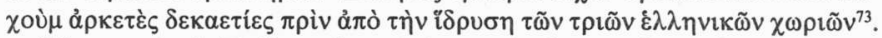

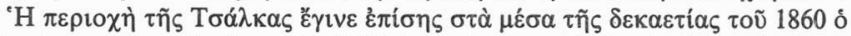

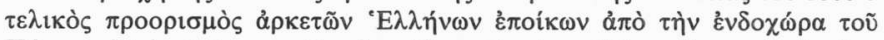

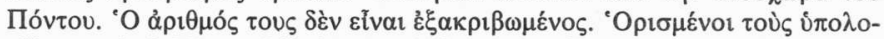

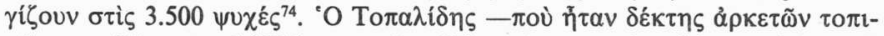

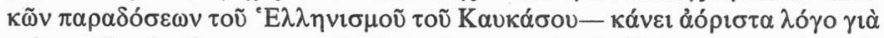

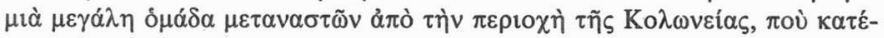

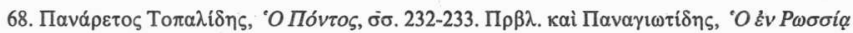

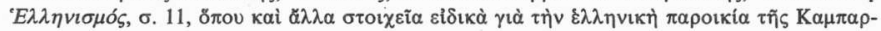

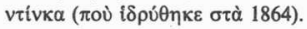

69. L. S. Lichkof, Ocherk iz proshlogo i nastoyashchego chernomorskogo poberezhya Kavkaza

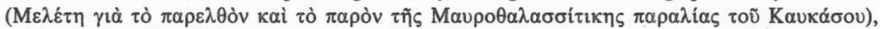

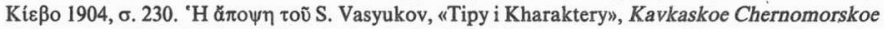

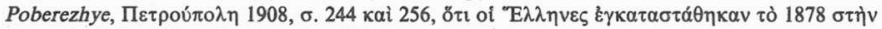

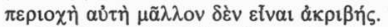

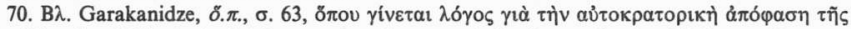

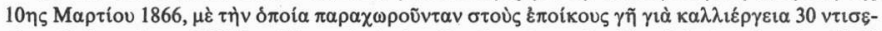

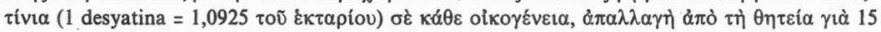

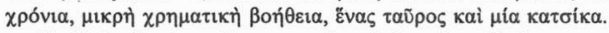

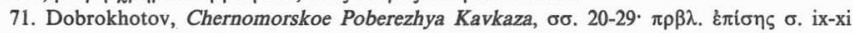
(Ei் $\alpha \gamma \omega \gamma \eta ́)$, ő

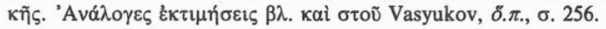

72. Garakanidze, ס̃. $\pi$., бб. 62-63.

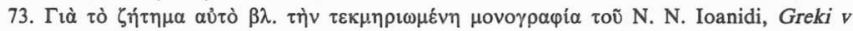

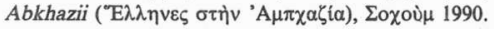

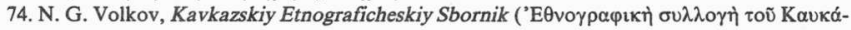
бov), Mó $\chi \alpha$ 1969, б. 7. 


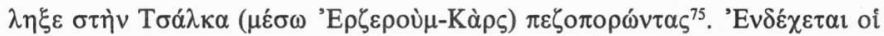

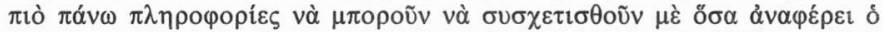

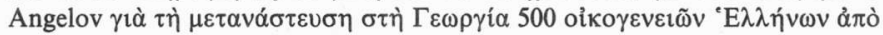

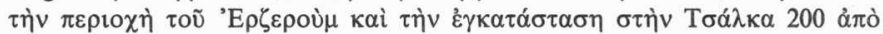

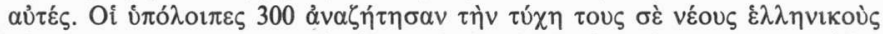
oỉkı

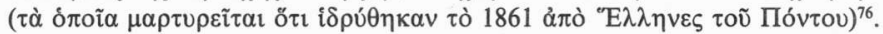

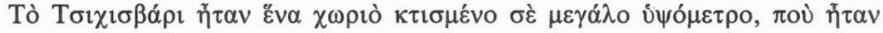

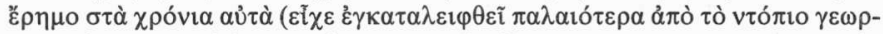

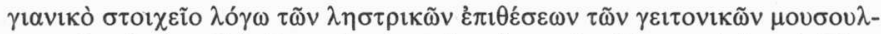

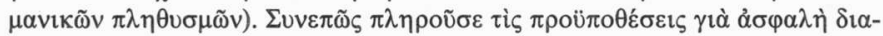

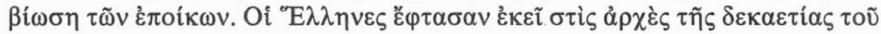

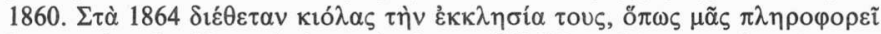

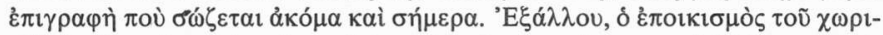

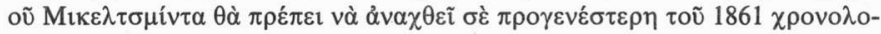

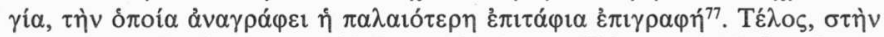

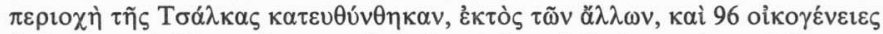

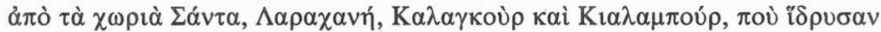

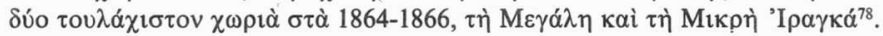

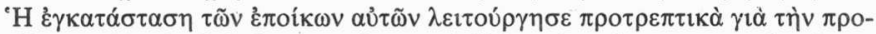

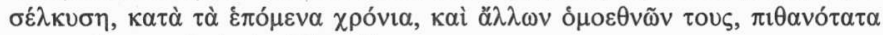

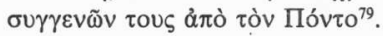

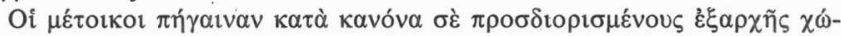

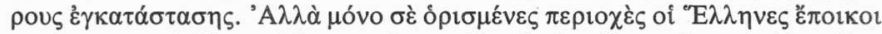

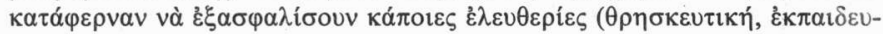

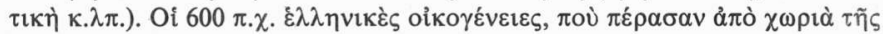

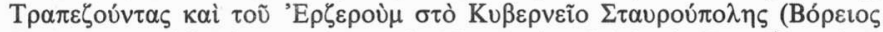

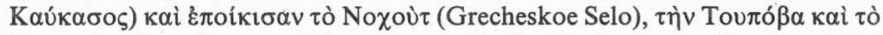

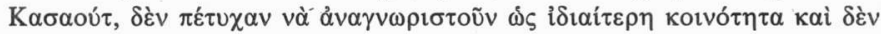

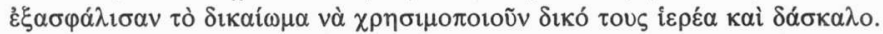

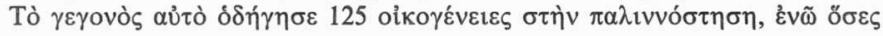

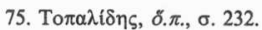

76. Angelov, $\delta . \pi ., \sigma .18$.

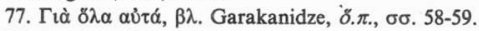

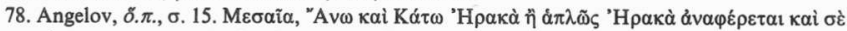

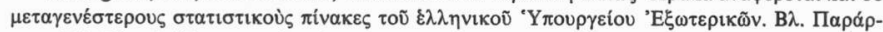

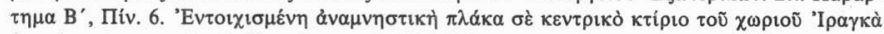

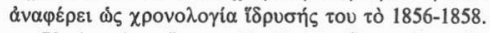

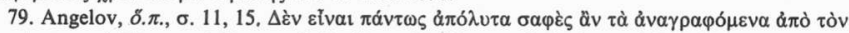

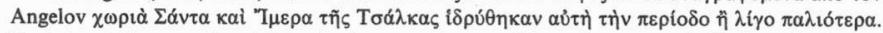

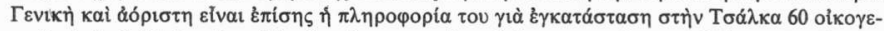

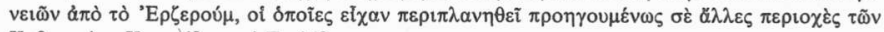

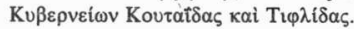




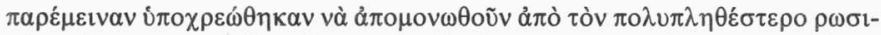

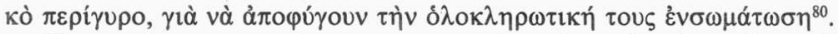

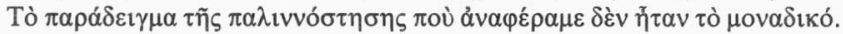

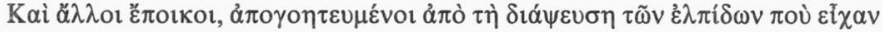

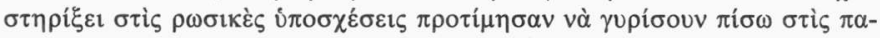

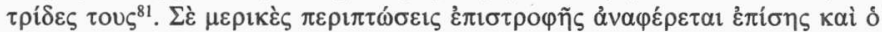

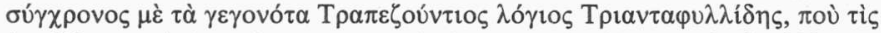

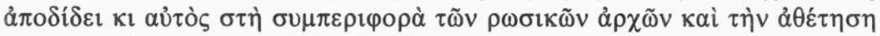

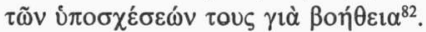

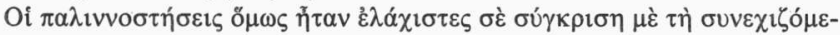

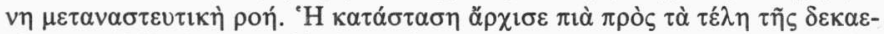

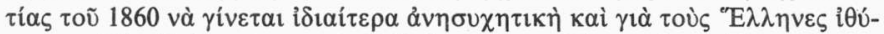

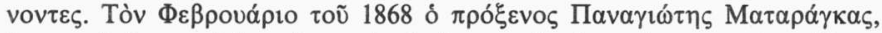

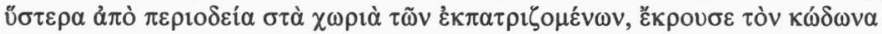

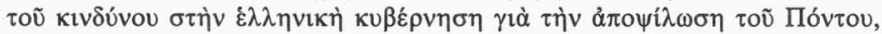

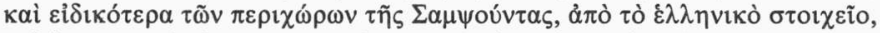

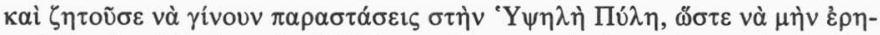

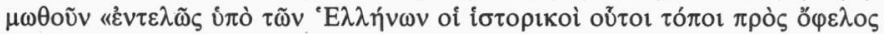

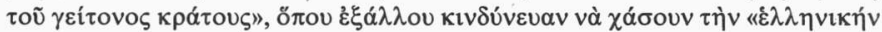

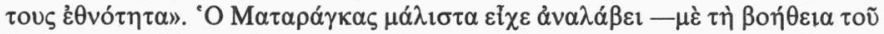

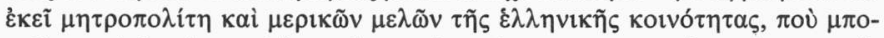

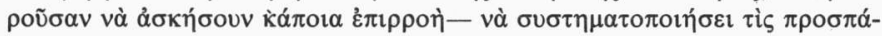

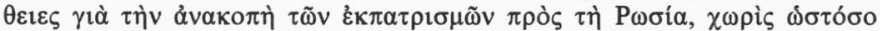

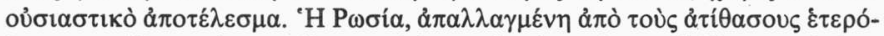

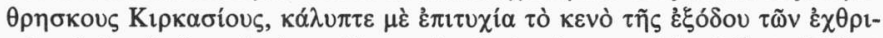

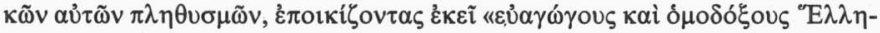

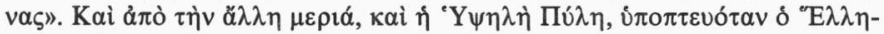

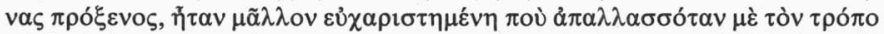

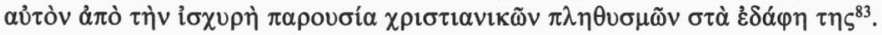

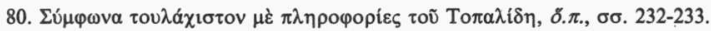

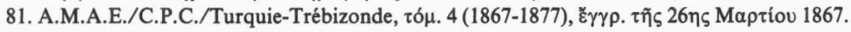

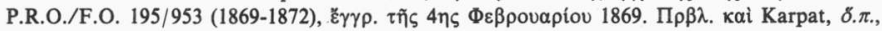
б. 68 .

82. Т $\rho \alpha v \tau \alpha \varphi v \lambda \lambda \hat{i} \delta \eta \varsigma, \Phi v \gamma \alpha ́ \delta \varepsilon \varsigma, \sigma .77$.

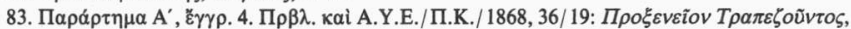

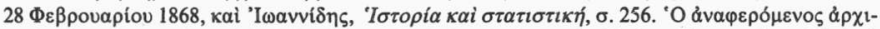

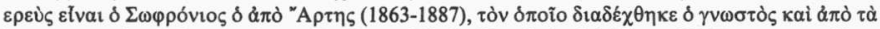

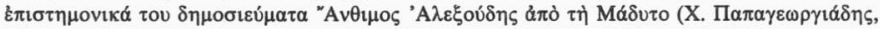

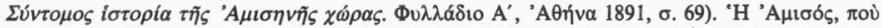

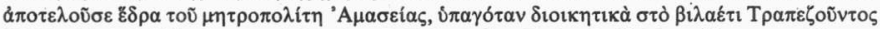

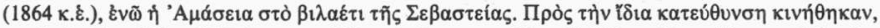

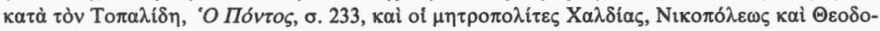




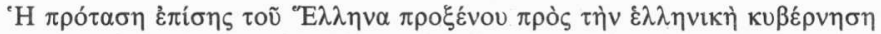

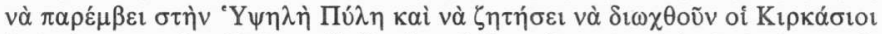

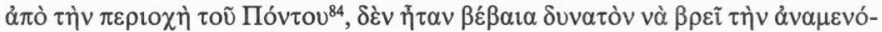

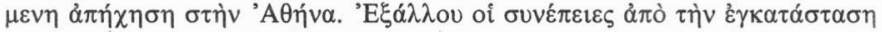

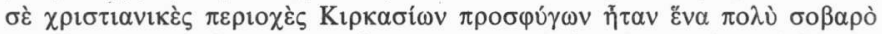

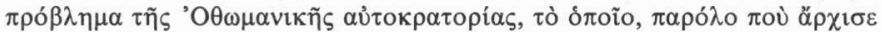

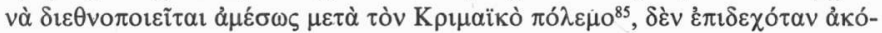

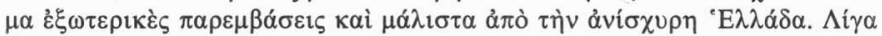

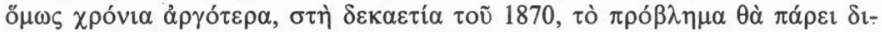

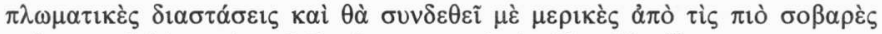

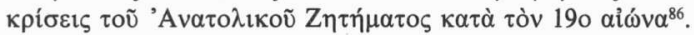

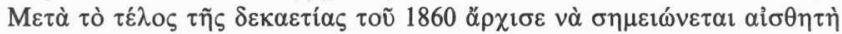

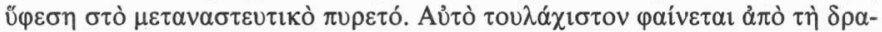

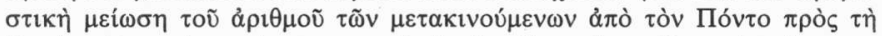

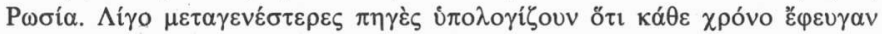

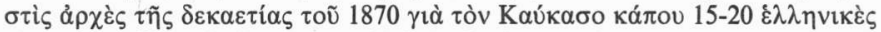

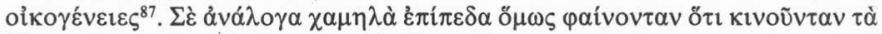

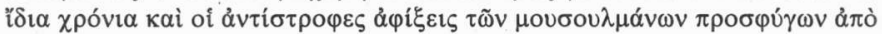

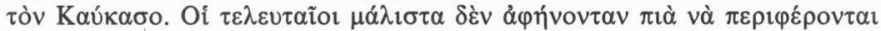

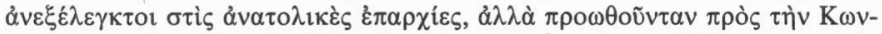

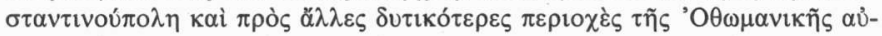

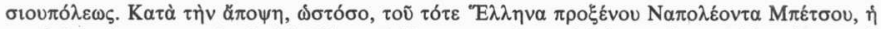

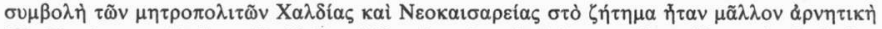

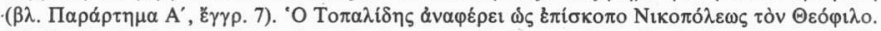

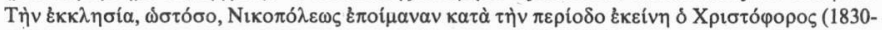

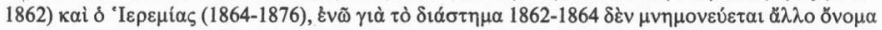

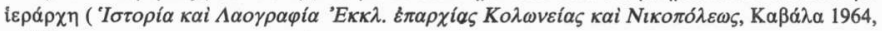
б. 20).

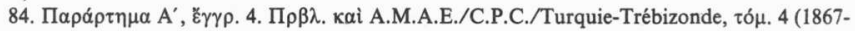

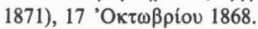
39-40.

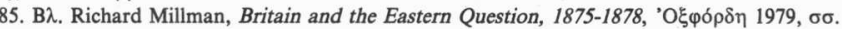

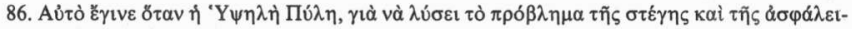

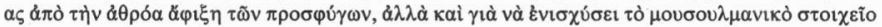

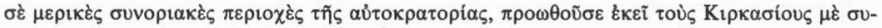

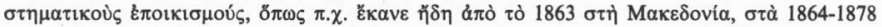

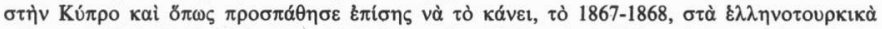

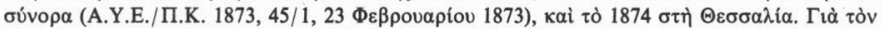

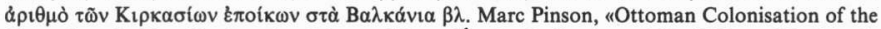
Circassians in Rumili after the Crimean War", Études Balkaniques 8/3, 1972, б. 72. Гì̀ tì

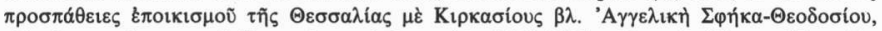
"'A

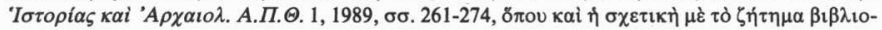

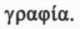

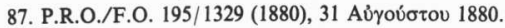




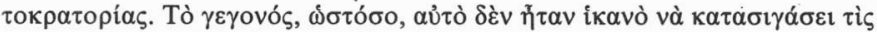

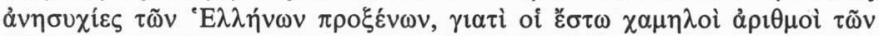

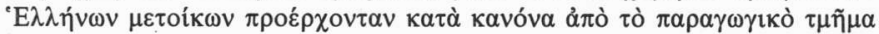

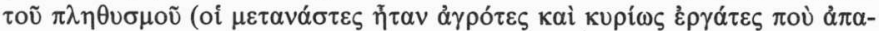

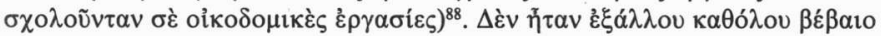

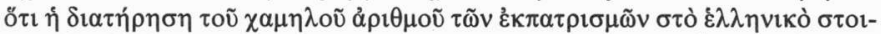

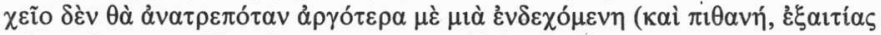

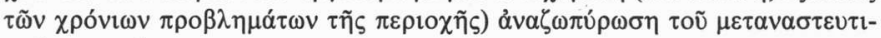
коธ̃ $\pi \nu \rho \varepsilon \tau о \tilde{\text {. }}$

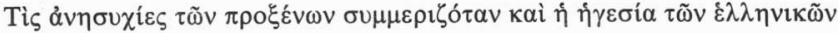

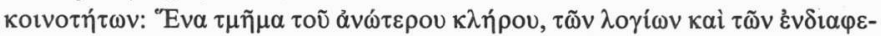

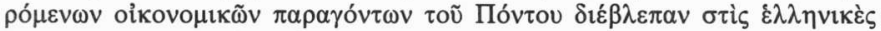

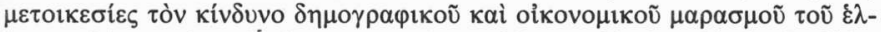

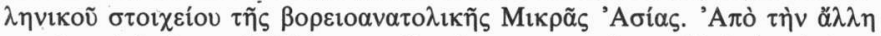

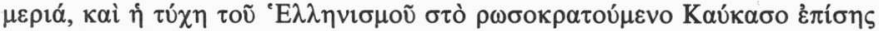

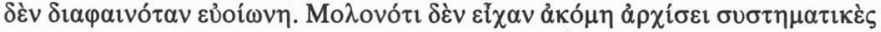

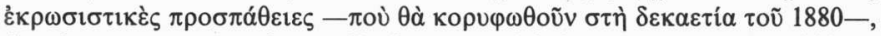

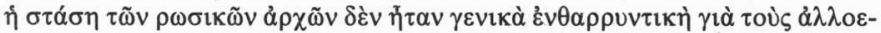

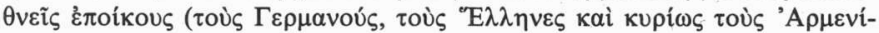

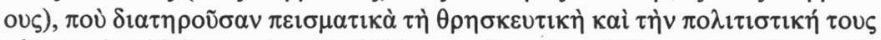

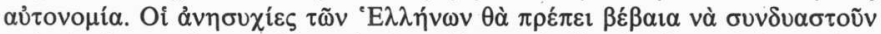

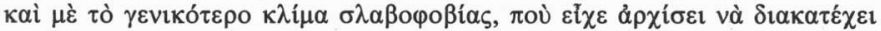

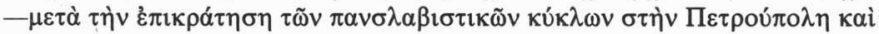

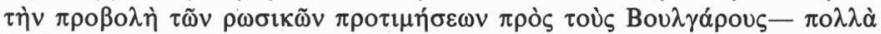

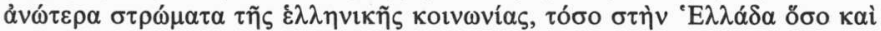

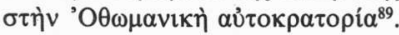

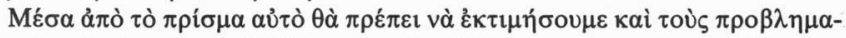

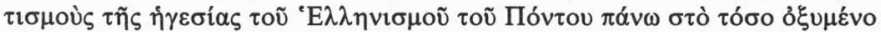

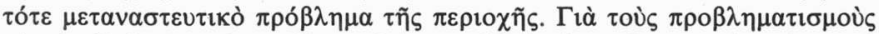

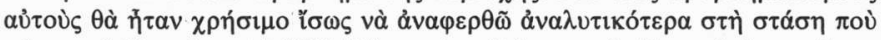

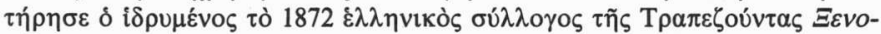

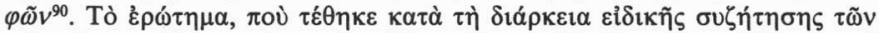

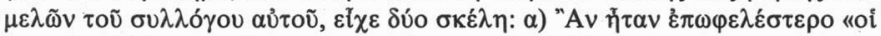

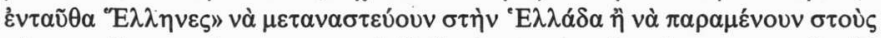

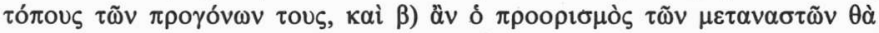

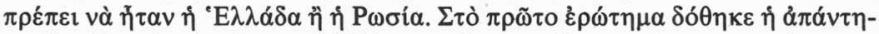

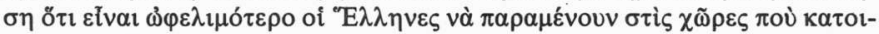

88. P.R.O./F.O. 195/953 (1869-1872), 7 Maptíou 1871.

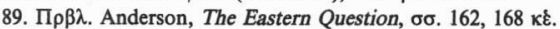

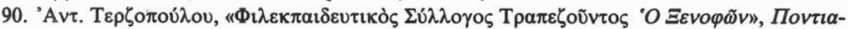

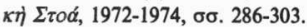




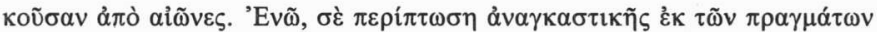

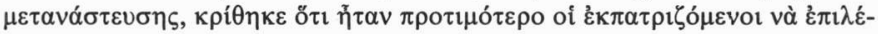

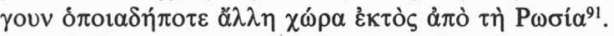

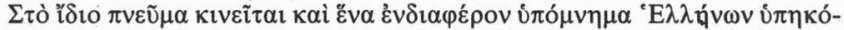

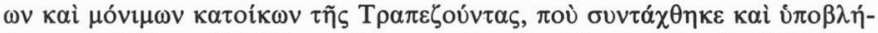

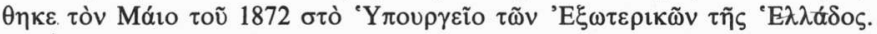

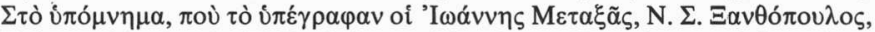

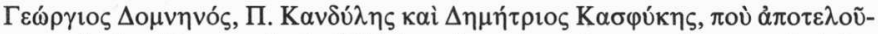

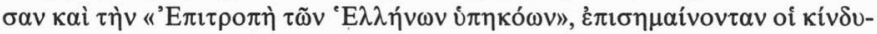

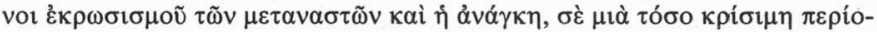

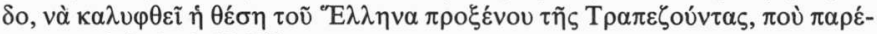

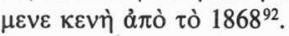

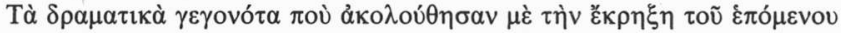

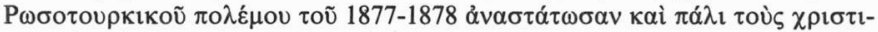

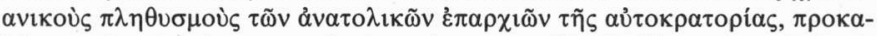

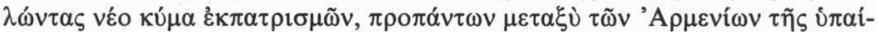

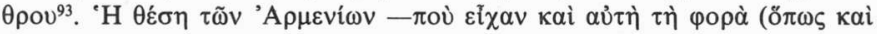

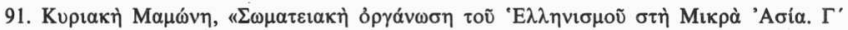

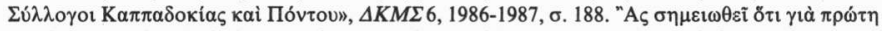

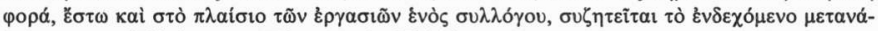

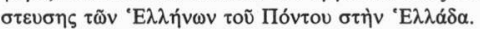

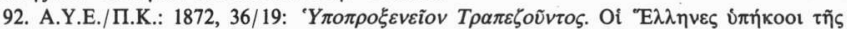

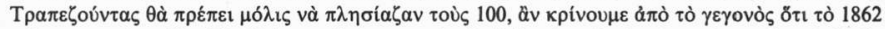

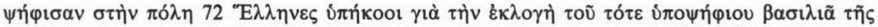

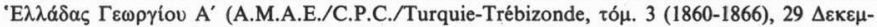

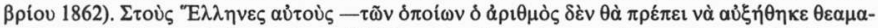

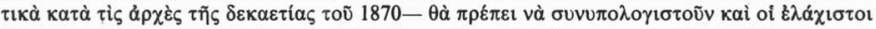

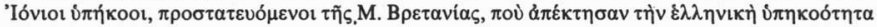

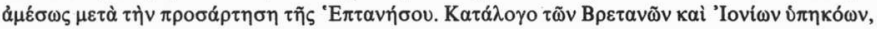

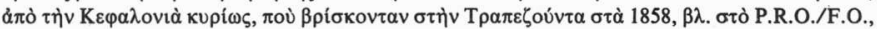

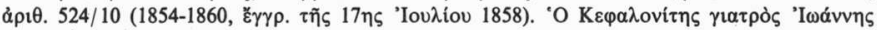

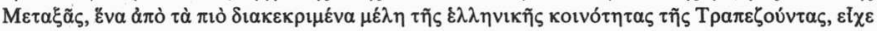

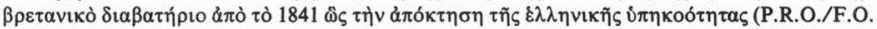

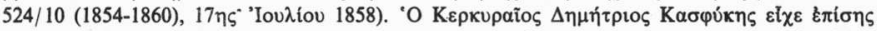

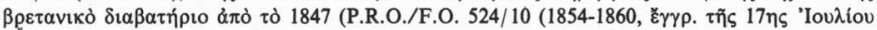

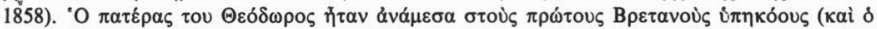

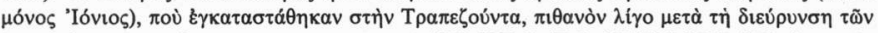

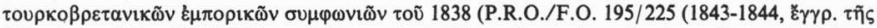

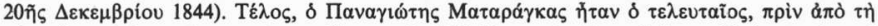

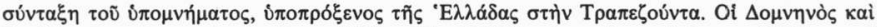

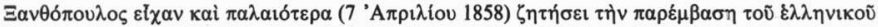
'Y

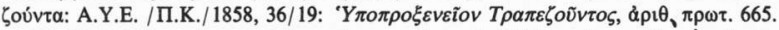

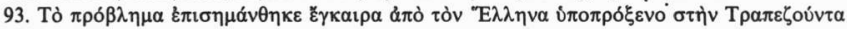

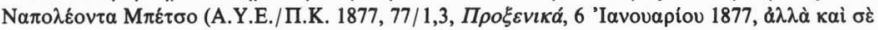




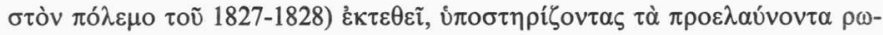

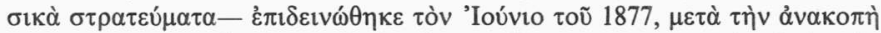

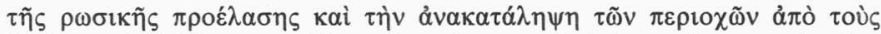

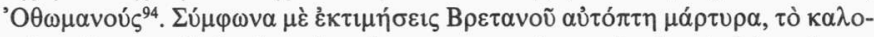

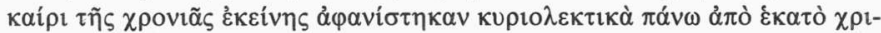

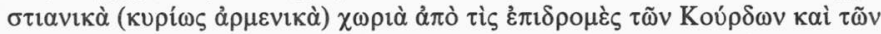

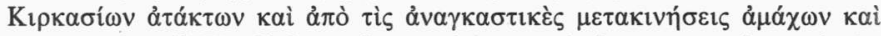

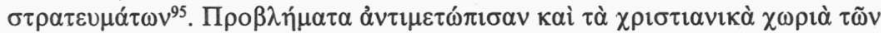

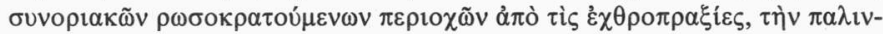

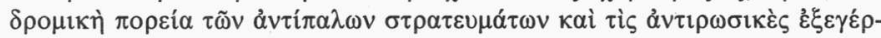

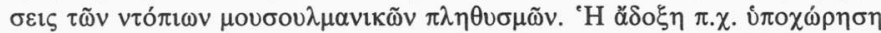

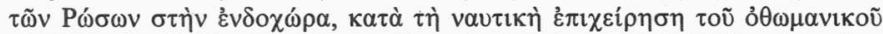

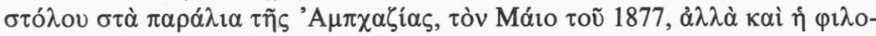

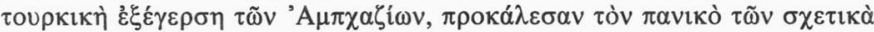

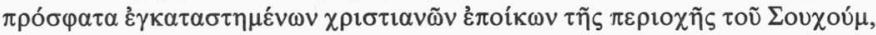

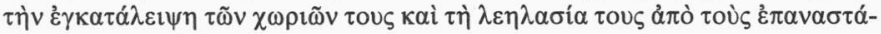

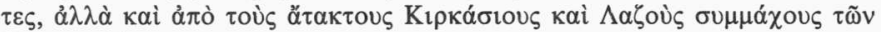

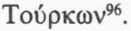

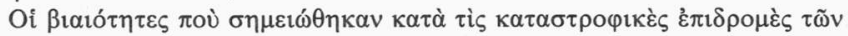

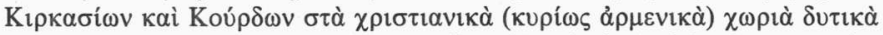

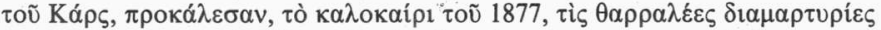

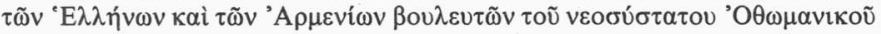

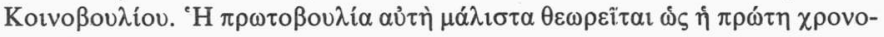

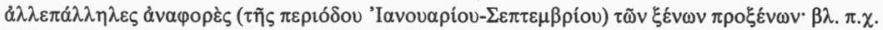

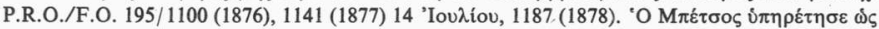

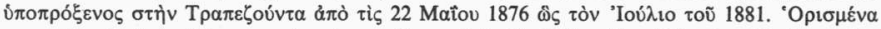

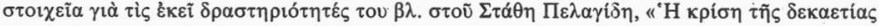

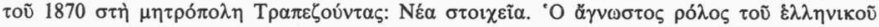

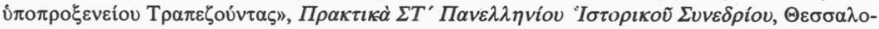
víkך 1986, бб. 79-89.

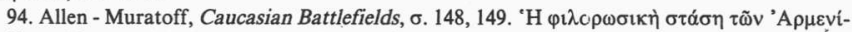

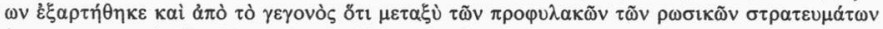

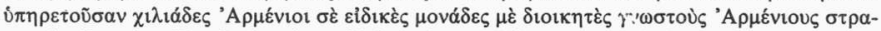

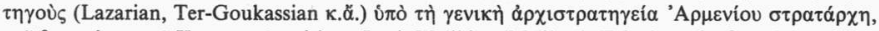

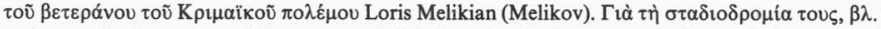

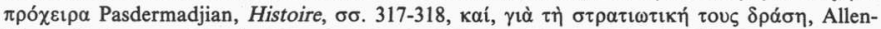

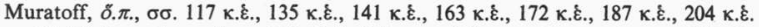

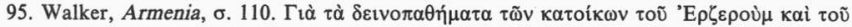

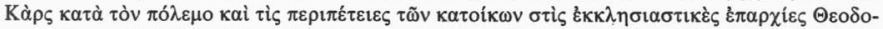

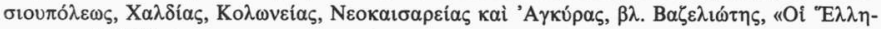

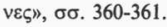

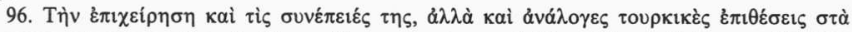

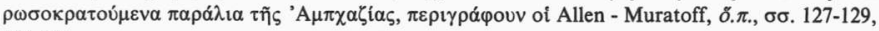
154-155. 


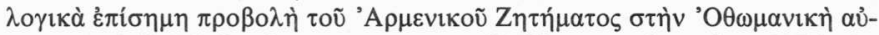

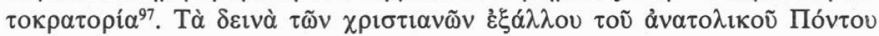

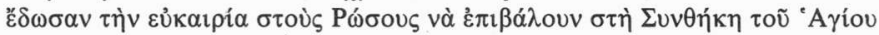

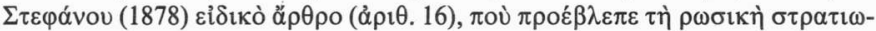

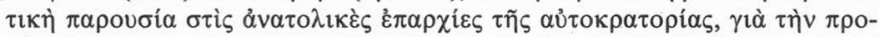

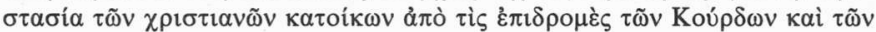

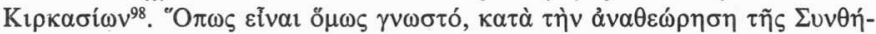

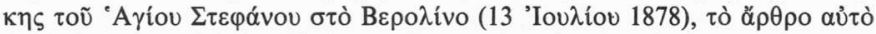

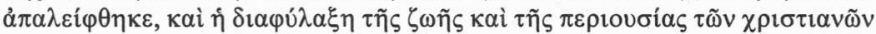

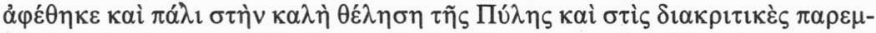

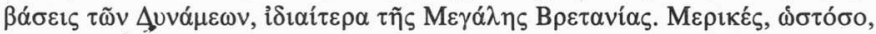

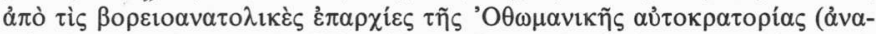

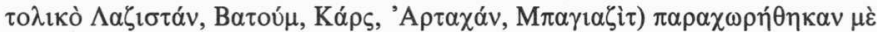

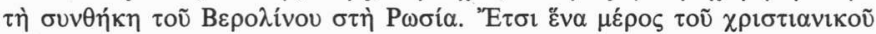

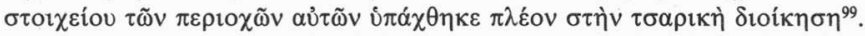

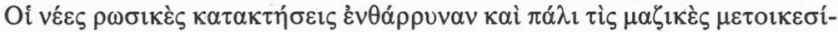

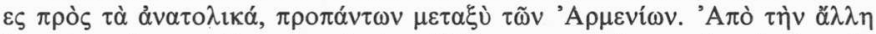

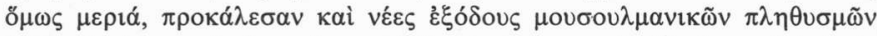
(ỉoí

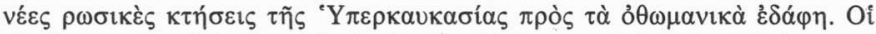

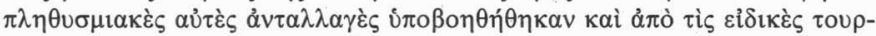

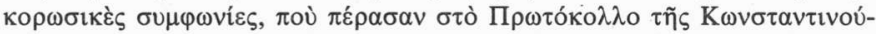

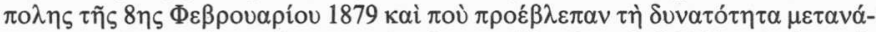

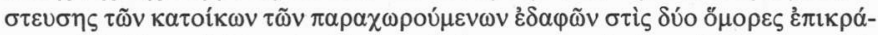

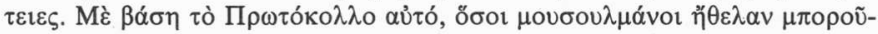

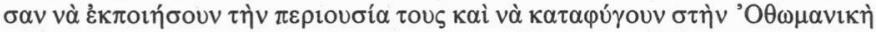

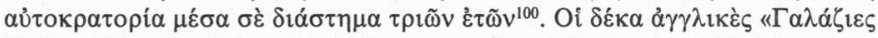

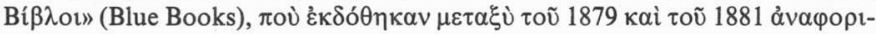

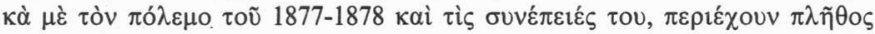

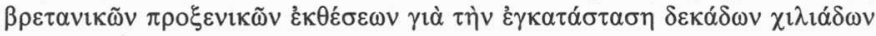

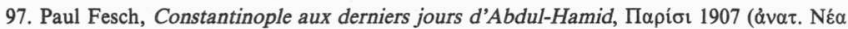

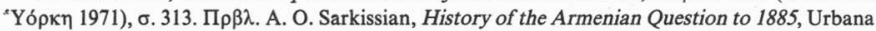

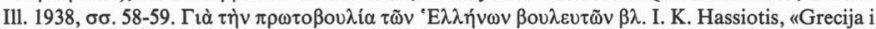

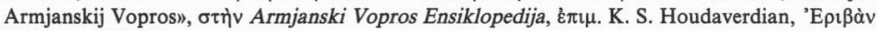
1991, бo. 152-153.

98. Walker, $\delta . \pi ., \sigma \sigma .111-112$.

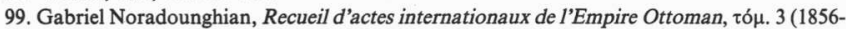

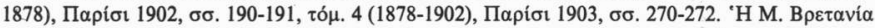

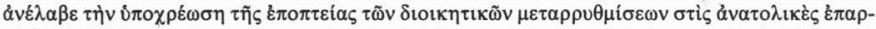

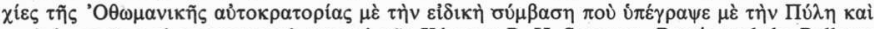

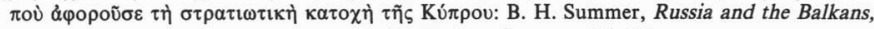

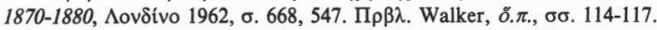

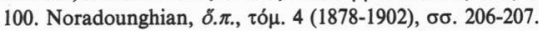




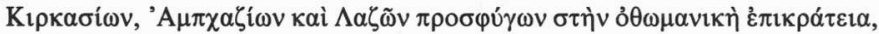

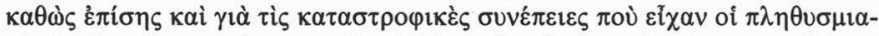

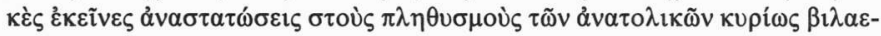

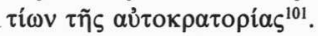

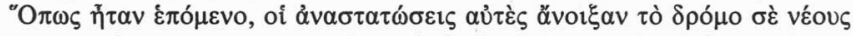

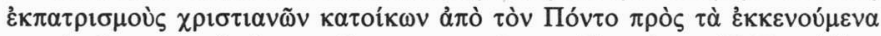

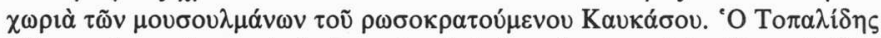

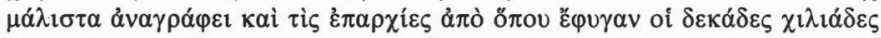

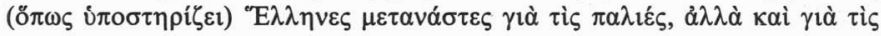

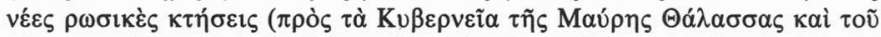

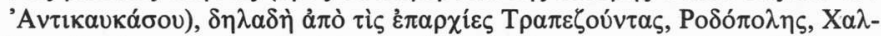

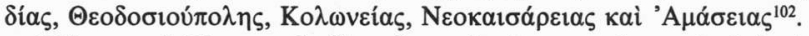

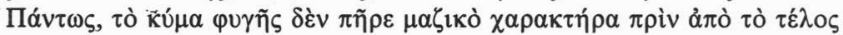

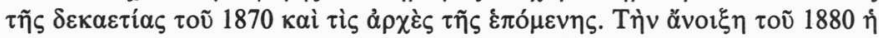

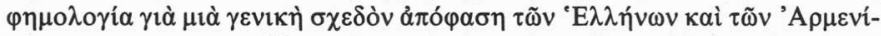

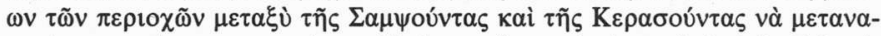

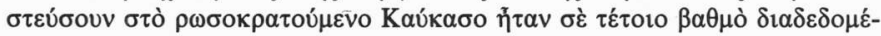

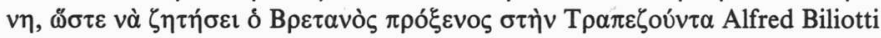

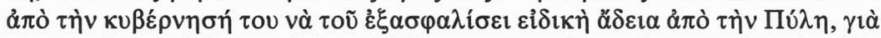

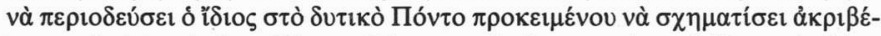

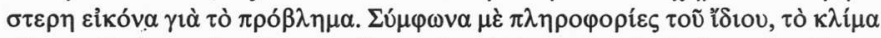

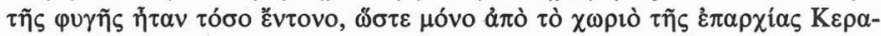

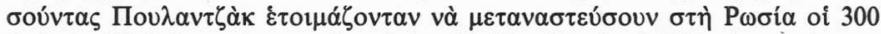

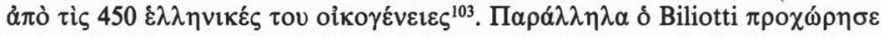

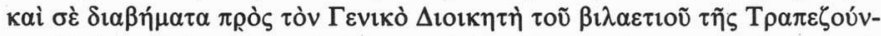

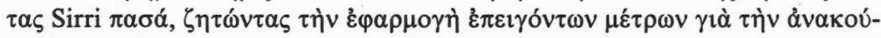

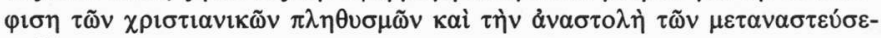
$\omega v^{104}$.

101. A.M.A.E./C.P.C./Turquie-Trébizonde, 5 (1878-1882) passim.

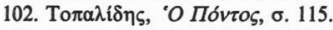

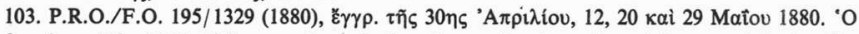

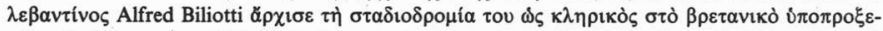

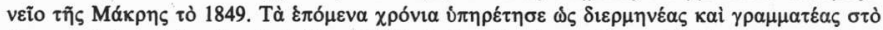

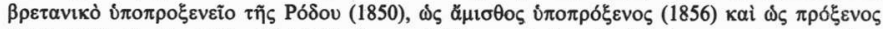

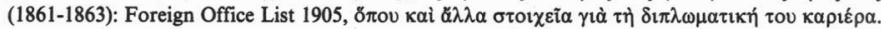

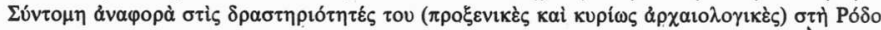

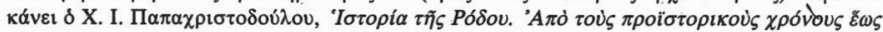

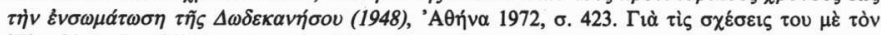

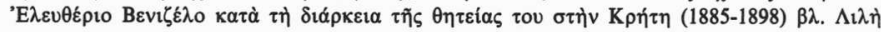

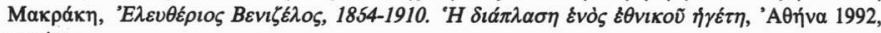
passim.

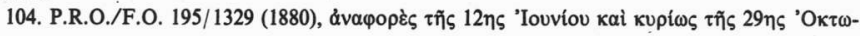




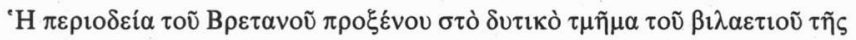

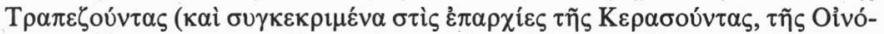

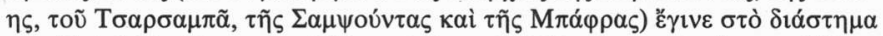

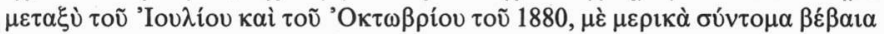

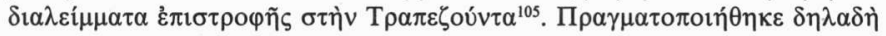

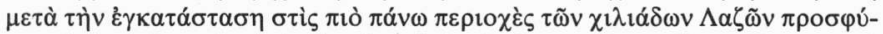

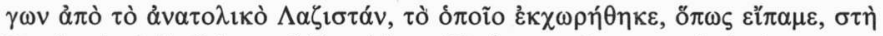

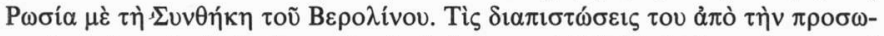

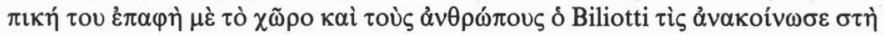

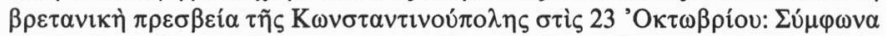

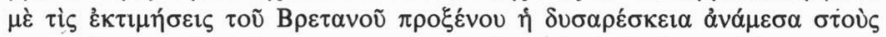

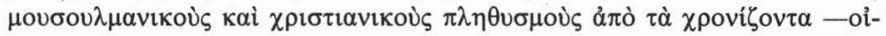

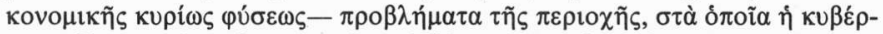

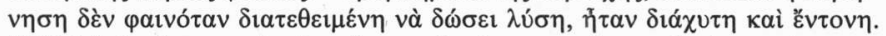

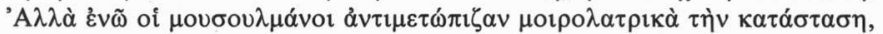

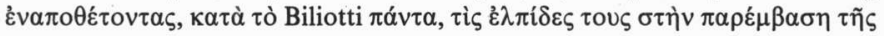

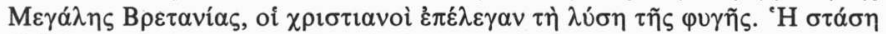

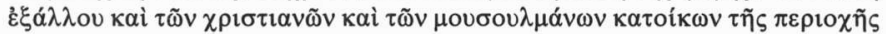

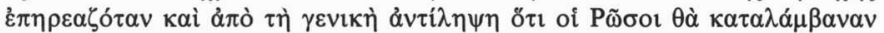

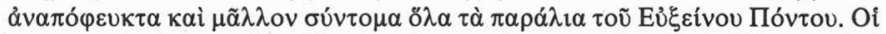

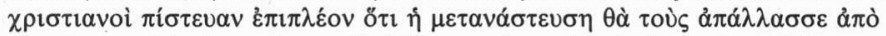

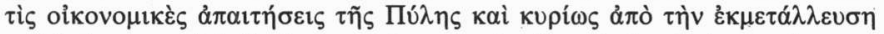

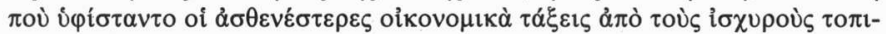

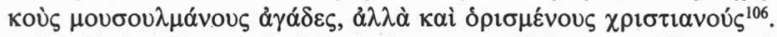

'Avád

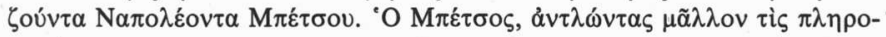

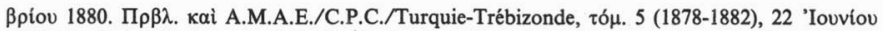

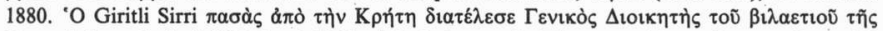

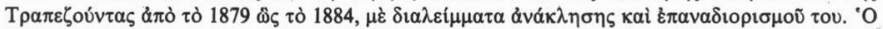

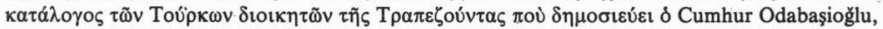

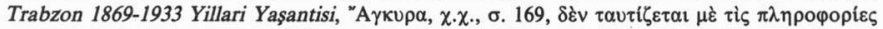

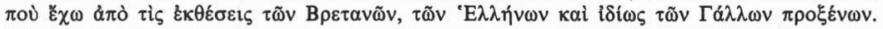

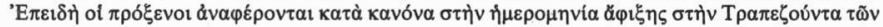

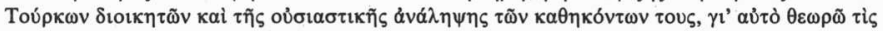

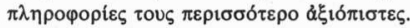

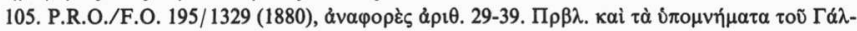

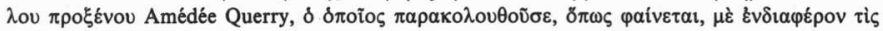

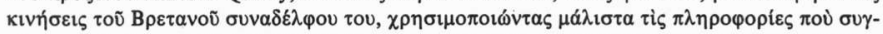

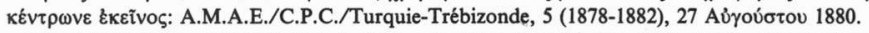

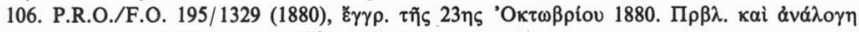

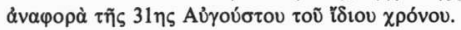




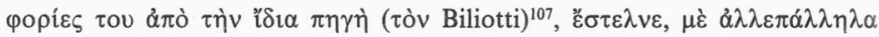

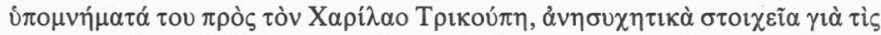

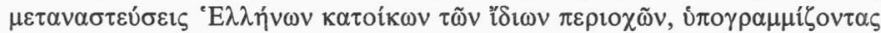

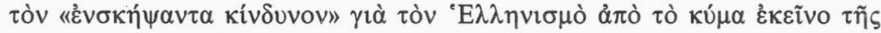

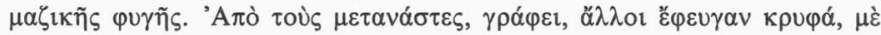

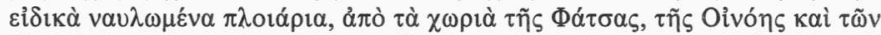

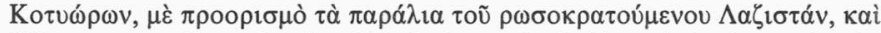

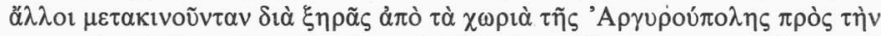

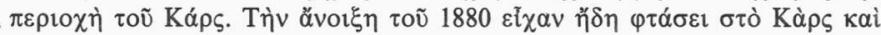

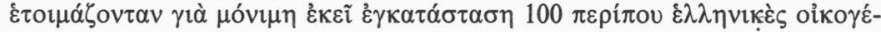
ve1є $\varsigma^{108}$.

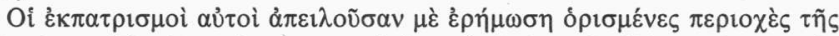

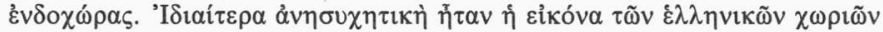

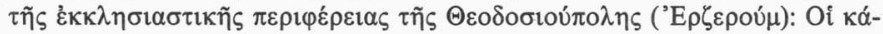

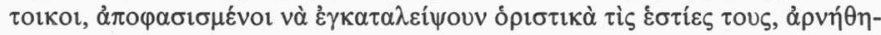

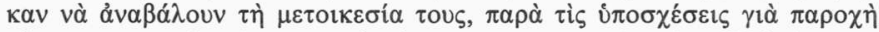

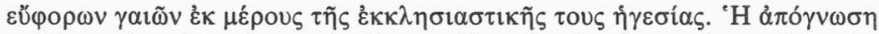

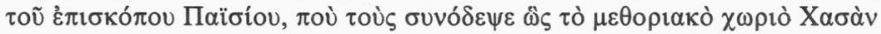

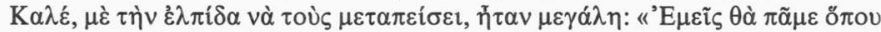

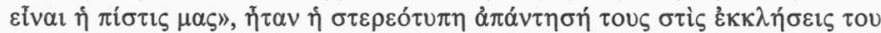

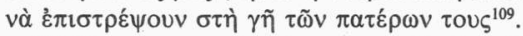

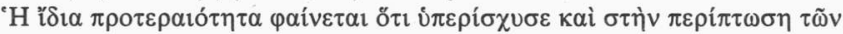

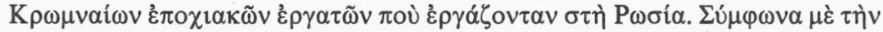

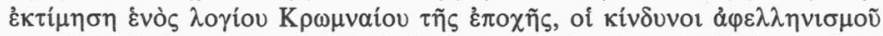

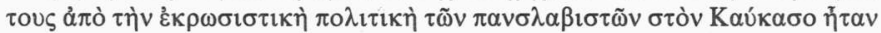

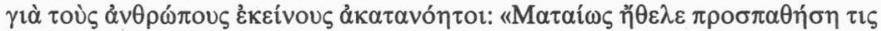
vò ảं

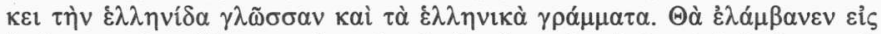

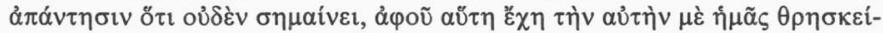
$\alpha v{ }^{110}$.

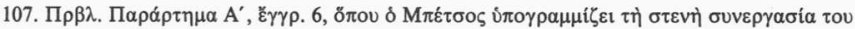
$\mu \varepsilon \dot{~ \tau o ̀ v ~ B i l i o t t i ~} \sigma \tau o ̀ ~ \pi \rho o ́ \beta \lambda \eta \mu \alpha ~ \tau \tilde{v ~} \mu \varepsilon \tau o เ \kappa \varepsilon \sigma เ \tilde{v}$.

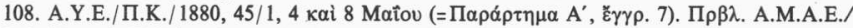
C.P.C./Turquie-Trébizonde, 5 (1878-1882), 25 Maiou kaì 4 'Iovvíov.

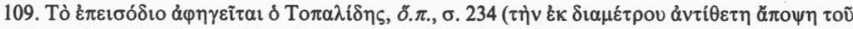

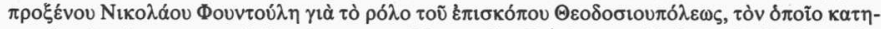

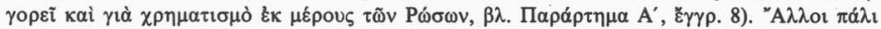

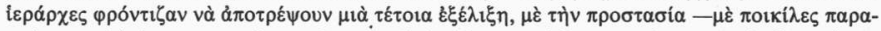

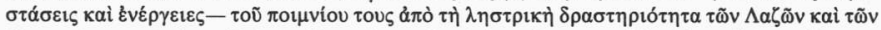

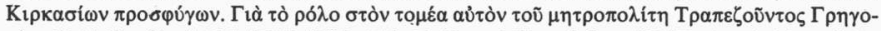

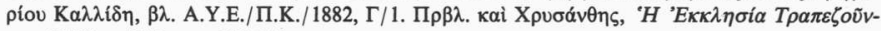

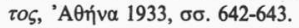

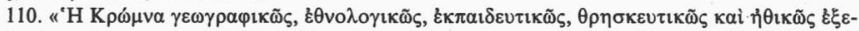

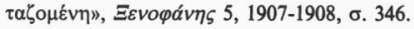




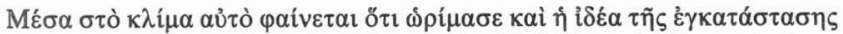

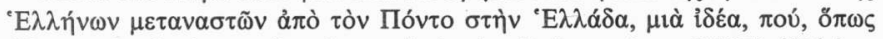

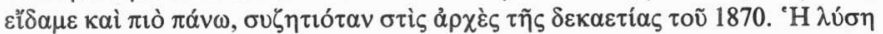

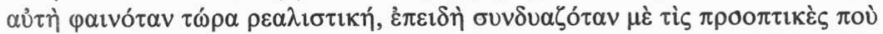

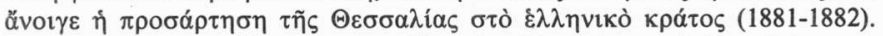

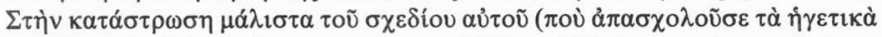

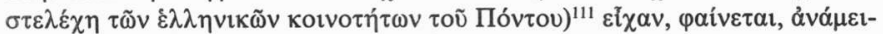

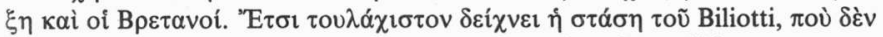

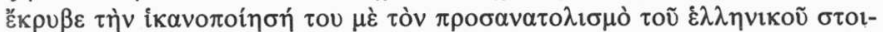

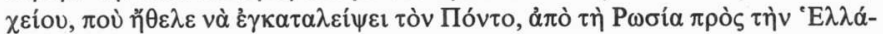

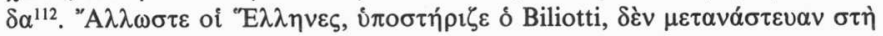

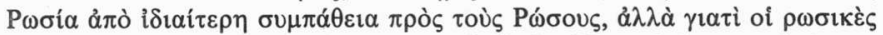

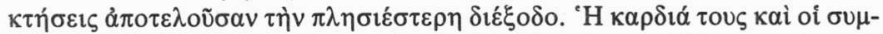

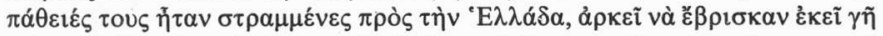

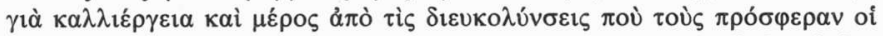

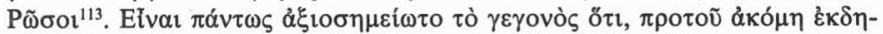

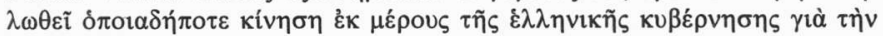

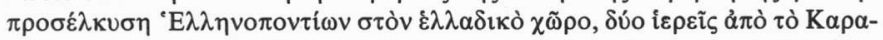

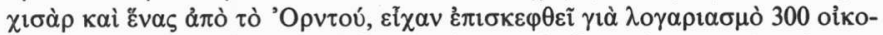

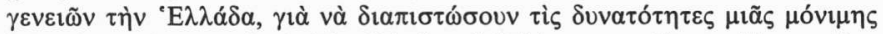

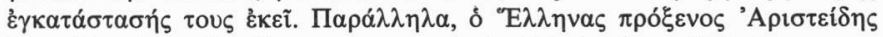

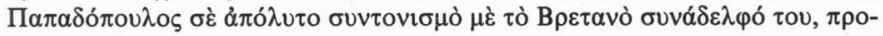

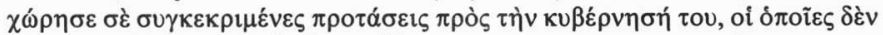

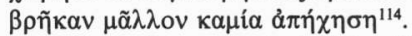

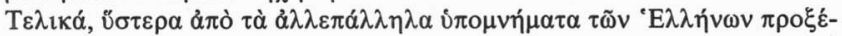

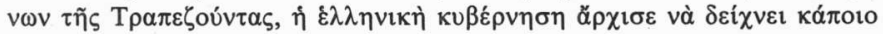

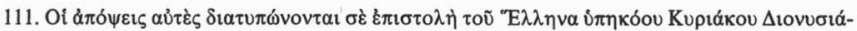

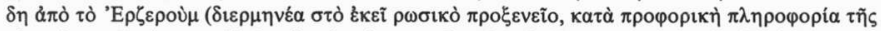

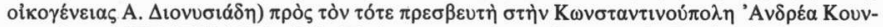

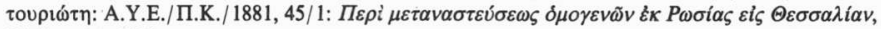
27 'Iouviou 1881.

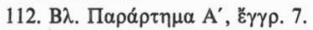

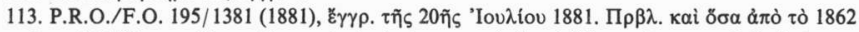

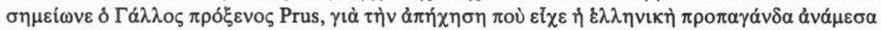

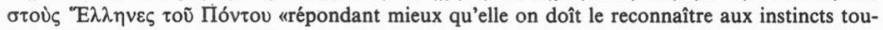
jours vivaces de nationalité d'un élément considérable de la population»: A.M.A.E./C.P.C./Tur-

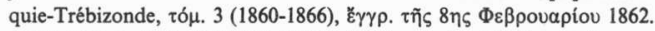

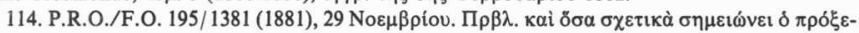

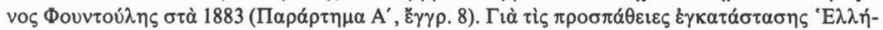

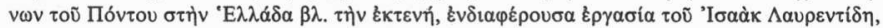

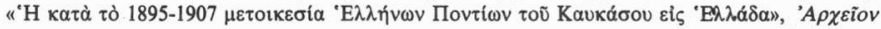

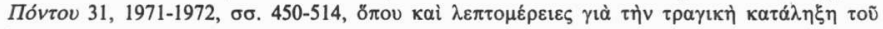

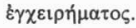




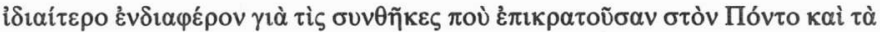

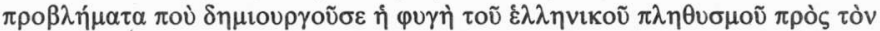

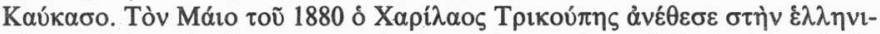

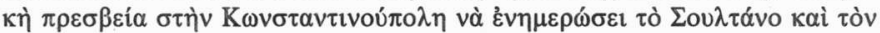

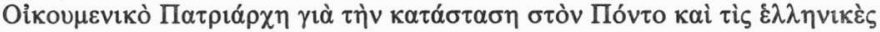

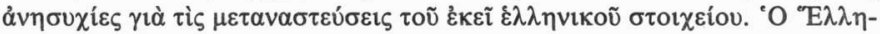

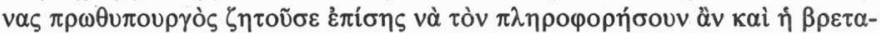

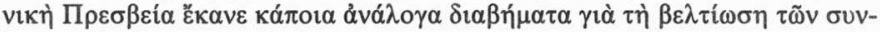
$\theta \eta \kappa \tilde{\omega} v \tau \tilde{\eta} \varsigma \zeta \omega \tilde{\eta} \varsigma \tau \tilde{\omega} v \pi \lambda \eta \theta v \sigma \mu \tilde{\omega} v \tau \tilde{\omega} v \pi \varepsilon \rho 10 \chi \tilde{\omega} v \alpha \boldsymbol{\nu}^{\tau} \tilde{\tilde{v}} v^{115}$.

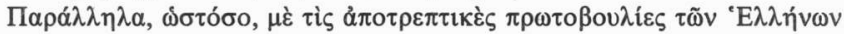

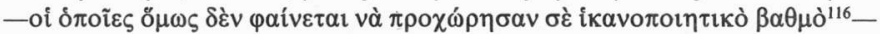

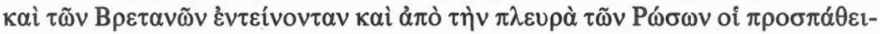

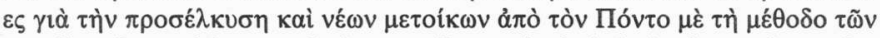

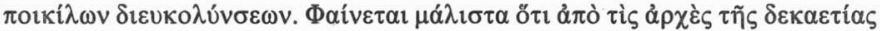

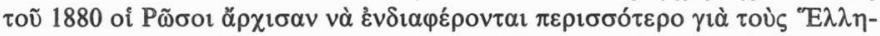

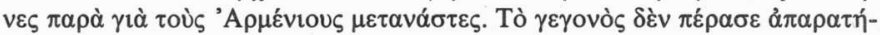

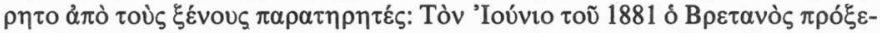

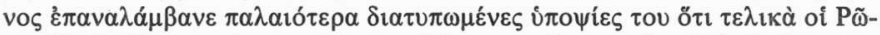

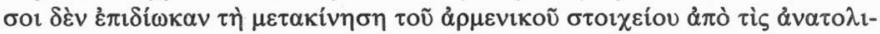

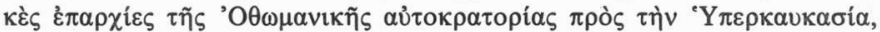

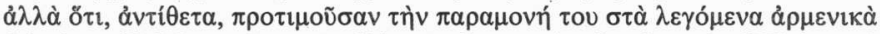

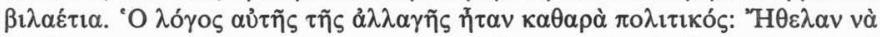

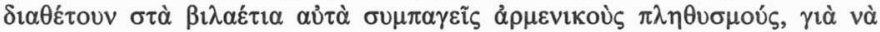

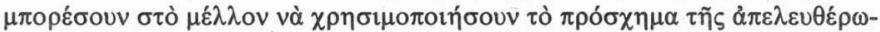

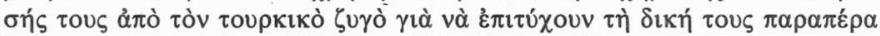

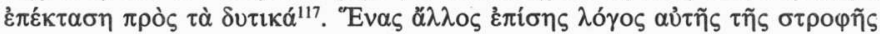

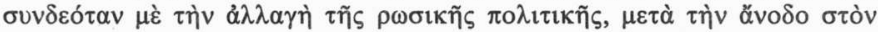

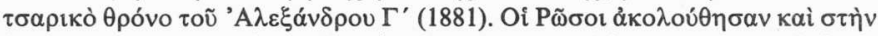

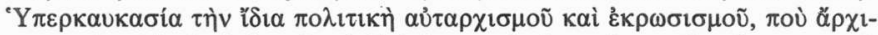

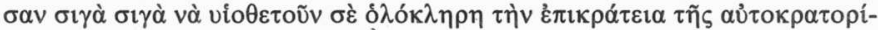

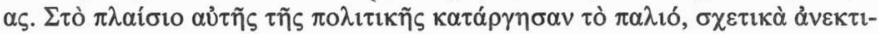

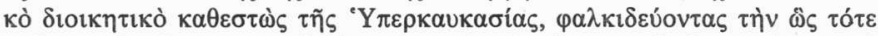

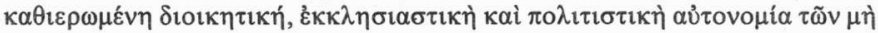

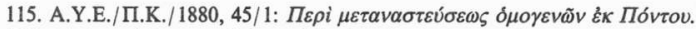

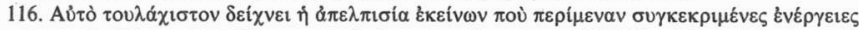

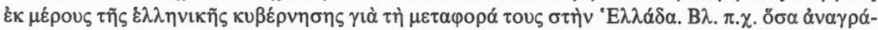

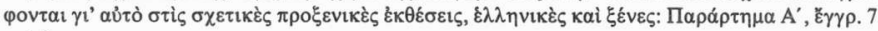
kaì 8.

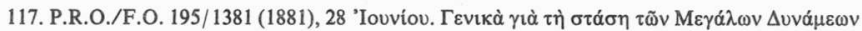

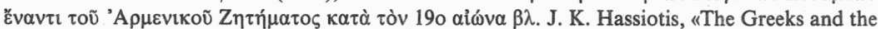

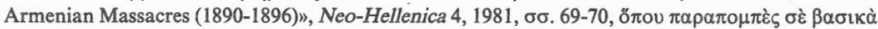

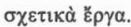




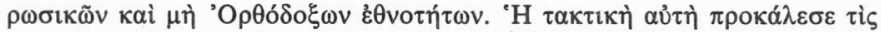

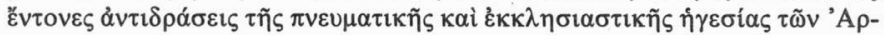

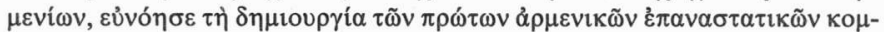

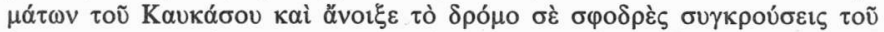

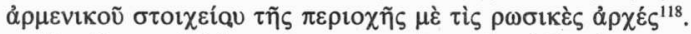

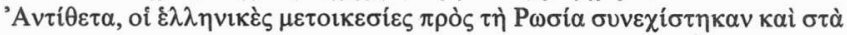

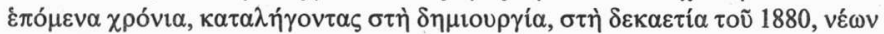

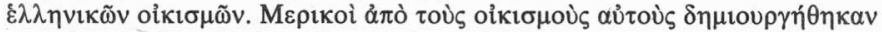

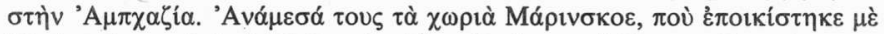

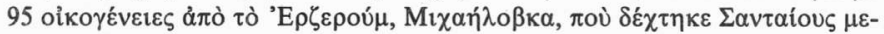

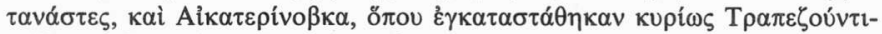

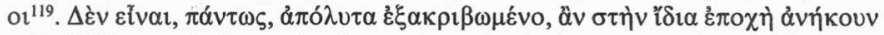

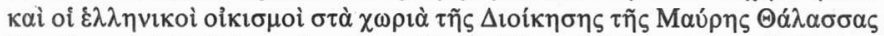

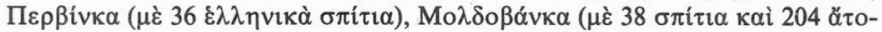

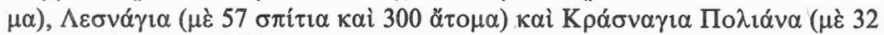

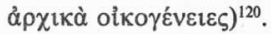

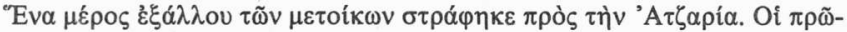

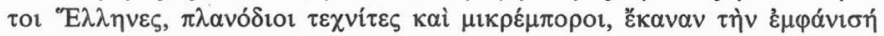

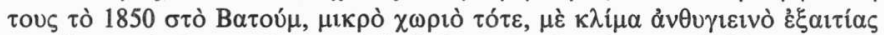

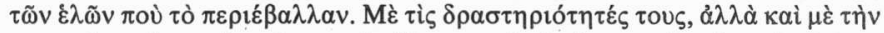

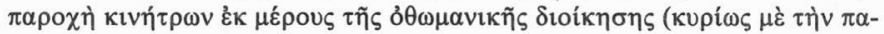


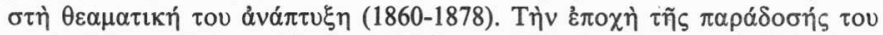

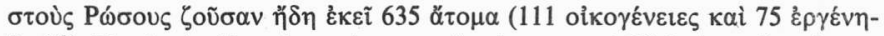

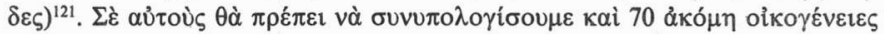

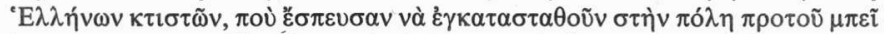

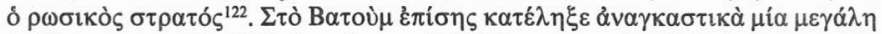

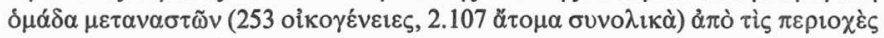

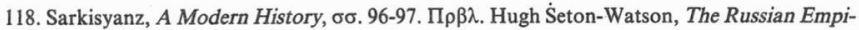

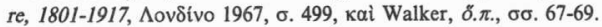

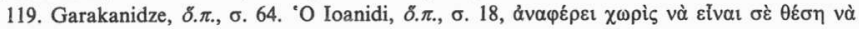

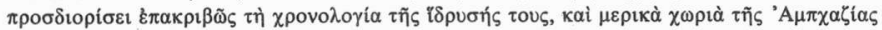

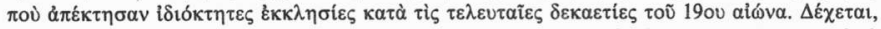

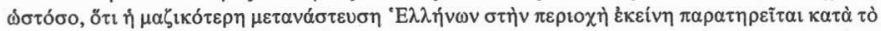
1880 .

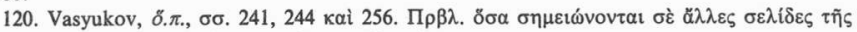

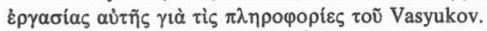

121. I. П. Káג $8-13,23$.

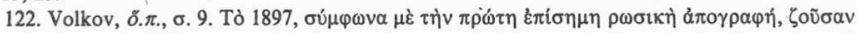

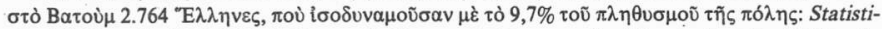

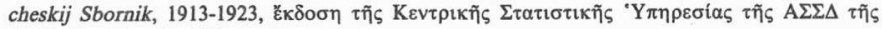

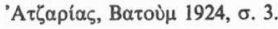




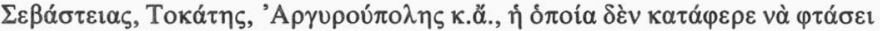

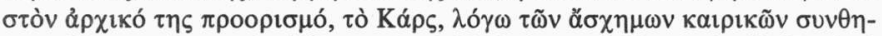

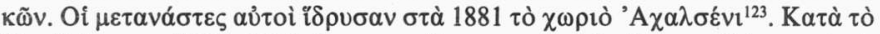

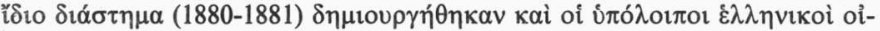

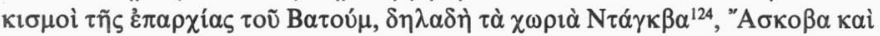

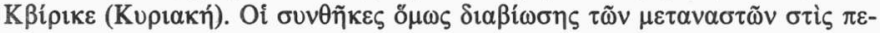

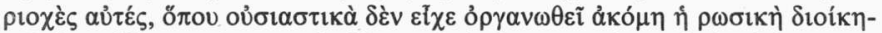

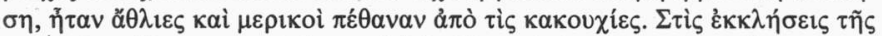

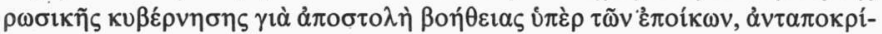

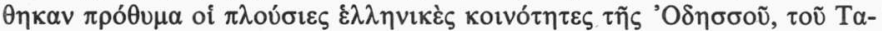

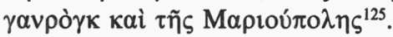

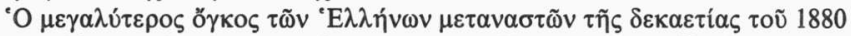

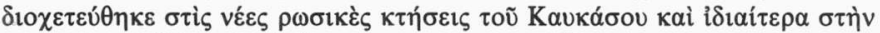

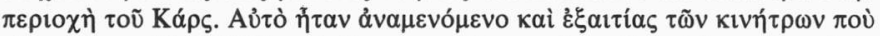

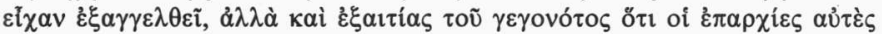

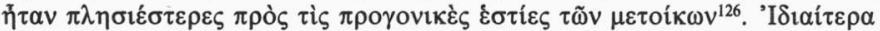

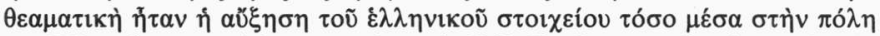

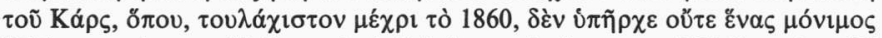

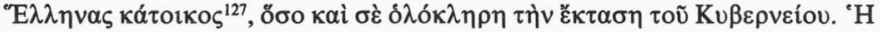

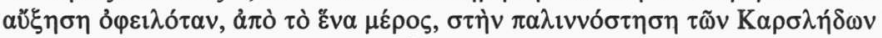

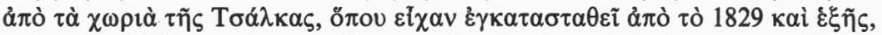

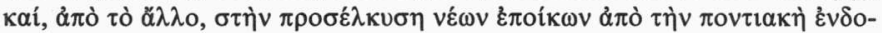
$\chi \omega \dot{\omega} \alpha^{128}$.

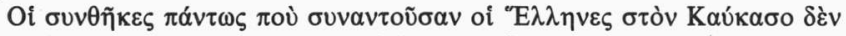

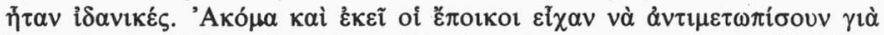

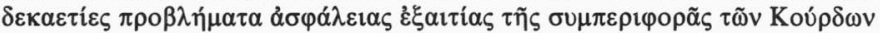

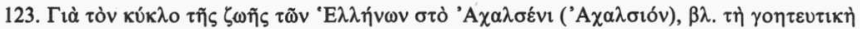

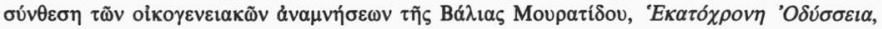

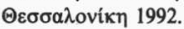

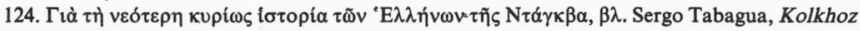

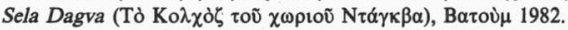

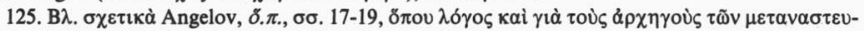

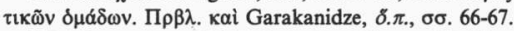

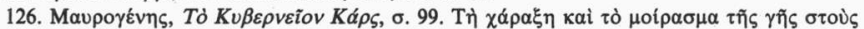

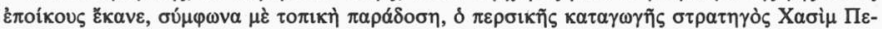

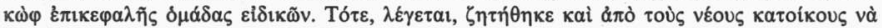

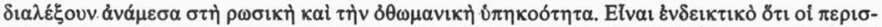

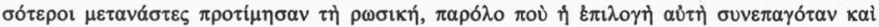

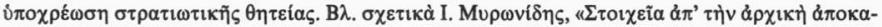

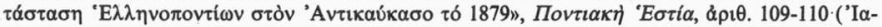

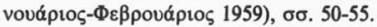

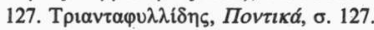

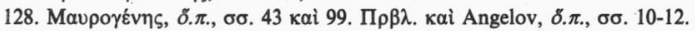




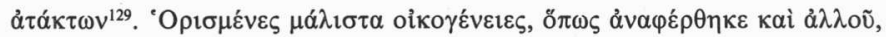

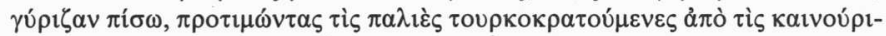

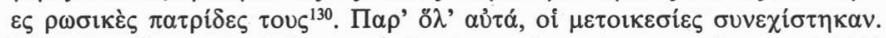

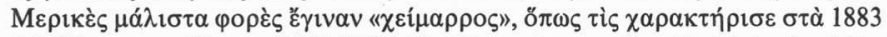

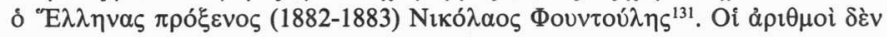

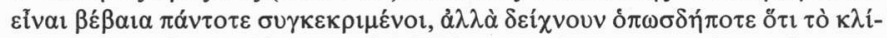

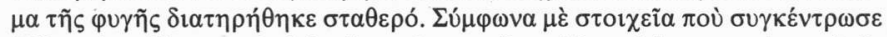

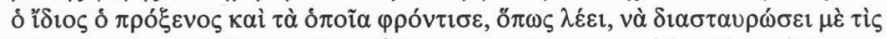

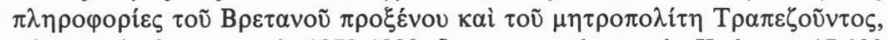

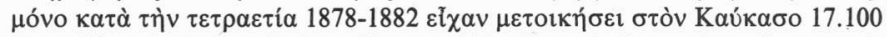

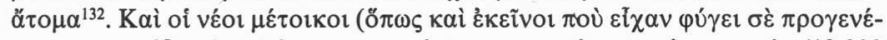

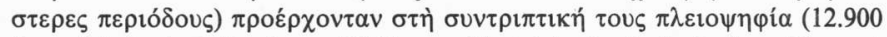

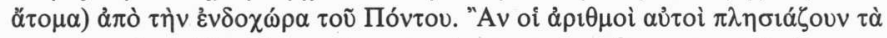

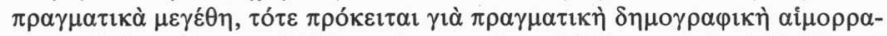

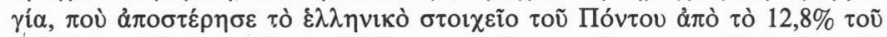

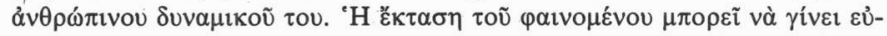

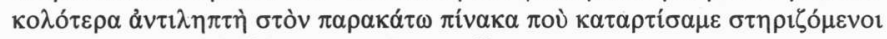

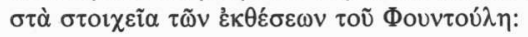

\begin{tabular}{|c|c|c|c|}
\hline$\pi \varepsilon \rho ı \chi \eta े ~$ & 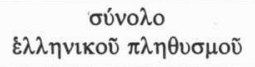 & $\begin{array}{c}\text { åp } \theta \mu \grave{o ̀ s} \\
\mu \varepsilon \tau \alpha \nu \alpha \sigma \tau \tilde{\omega} v\end{array}$ & $\pi \circ \sigma \circ \sigma \tau \grave{\alpha} \%$ \\
\hline 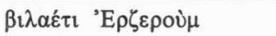 & 8.509 & 1.800 & 21,15 \\
\hline$\varepsilon \dot{\pi} \alpha \rho \chi i \alpha \quad \Sigma \varepsilon \beta a ́ \sigma \tau \varepsilon i \alpha \varsigma$ & 5.872 & 3.900 & 66,40 \\
\hline 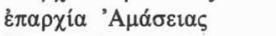 & 10.577 & 1.800 & 17 \\
\hline \multicolumn{4}{|l|}{ 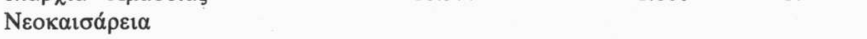 } \\
\hline 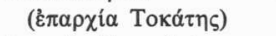 & 5.148 & 2.400 & 46,62 \\
\hline 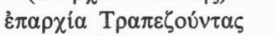 & 72.400 & 5.400 & 7,45 \\
\hline 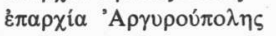 & 31.202 & 1.800 & 5,77 \\
\hline$\Sigma u ́ v o \lambda \alpha$ & 133.708 & 17.100 & 12,79 \\
\hline
\end{tabular}

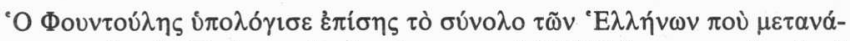

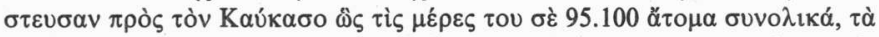

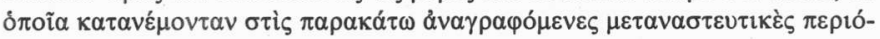

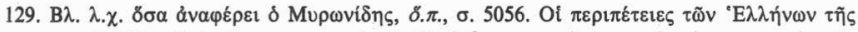

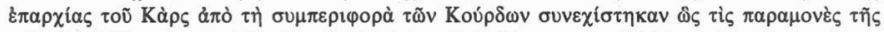

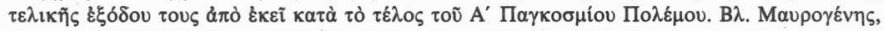

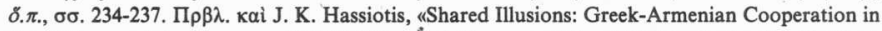
Asia Minor and the Caucasus (1917-1922)", 'otó: Greece and Great Britain during World War I,

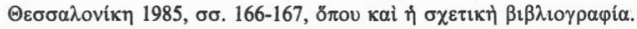

130. P.R.O./F.O. 195//1381 (1881), $3 \Delta \varepsilon \kappa \varepsilon \mu \beta$ píov 1881.

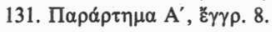

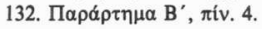




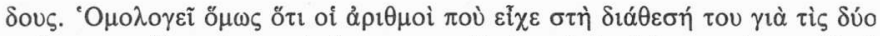

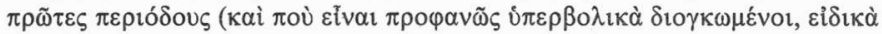

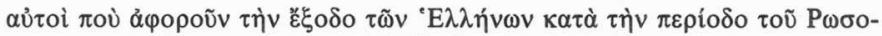

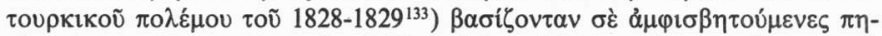

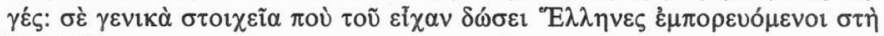
P $\omega \sigma i \alpha^{134}$ :

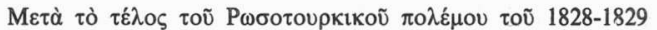

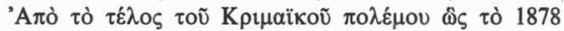

42.000 ă

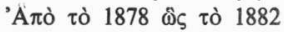

36.000 "

$17.100 》$

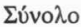

95.100 ăนо $\mu \alpha$

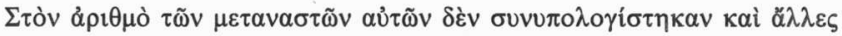

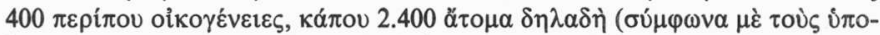

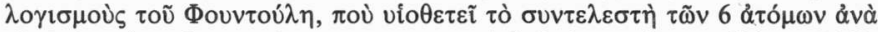

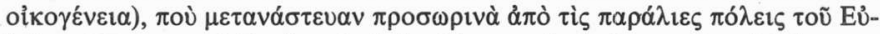

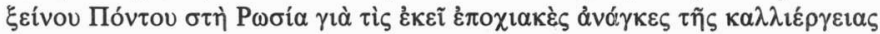

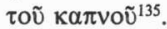

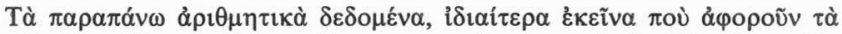

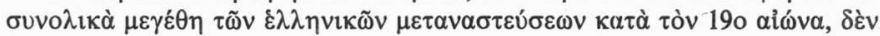

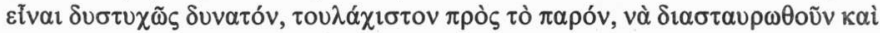

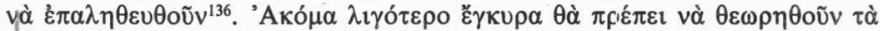

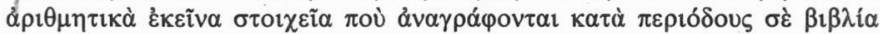

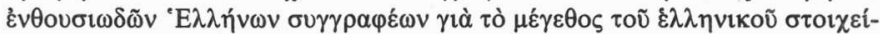

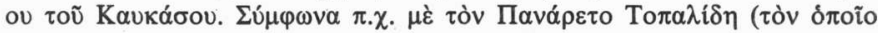

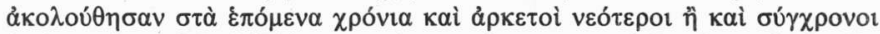

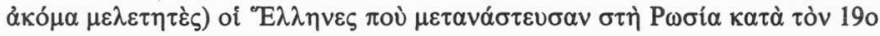

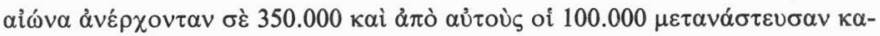

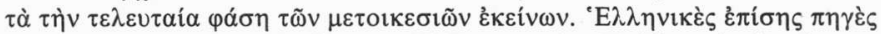

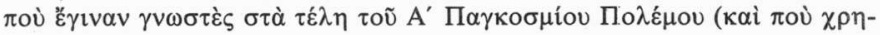

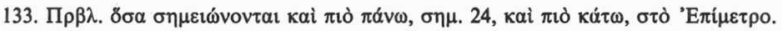

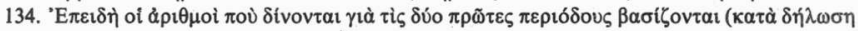

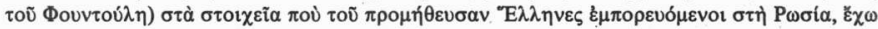

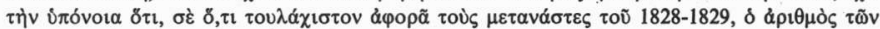

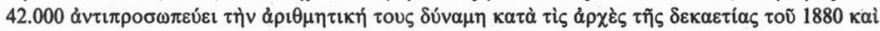

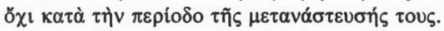

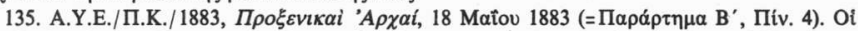

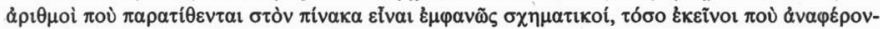

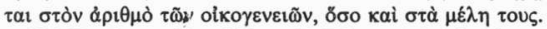

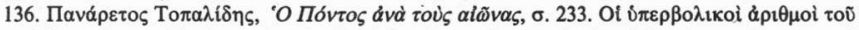

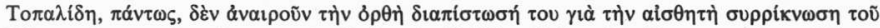

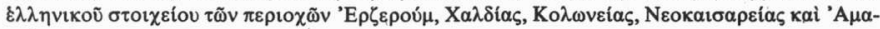

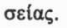




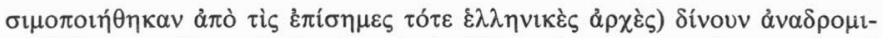

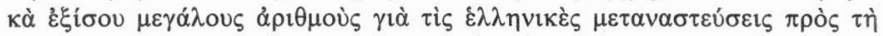

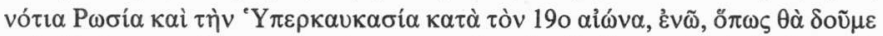

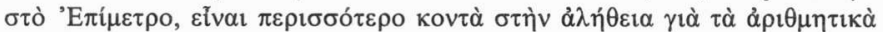

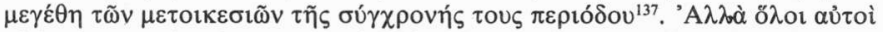

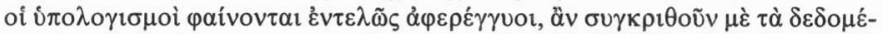

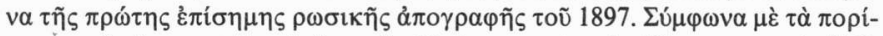
$\sigma \mu \alpha \tau \alpha \tau \tilde{\eta} \varsigma$ ảं

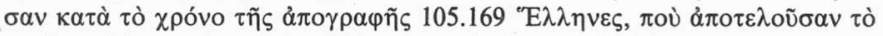

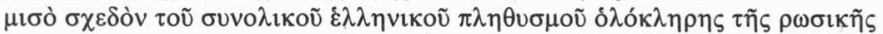

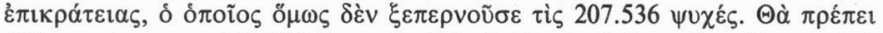

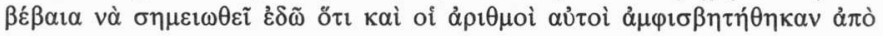

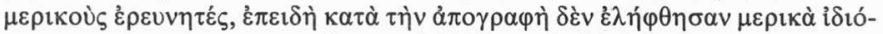

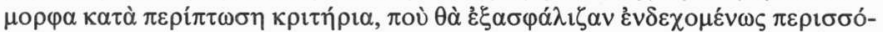

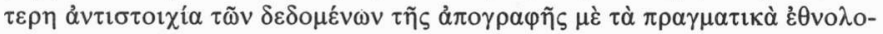

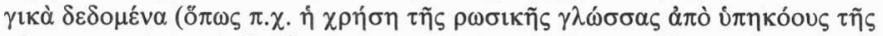

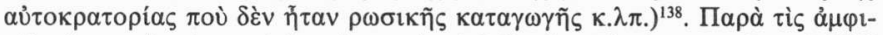

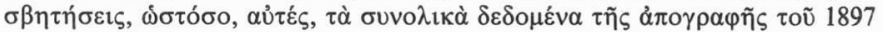

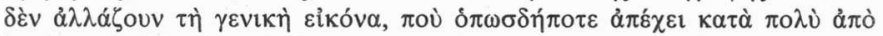

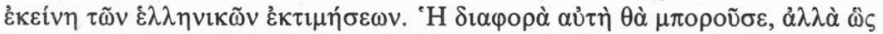

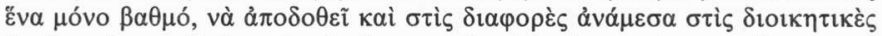

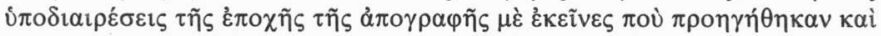

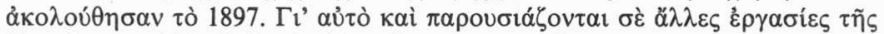

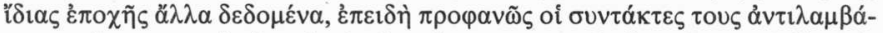

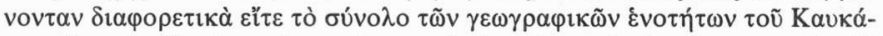

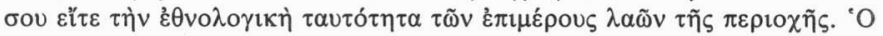

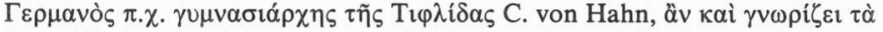

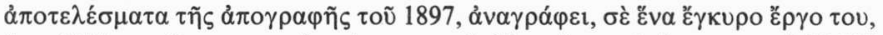

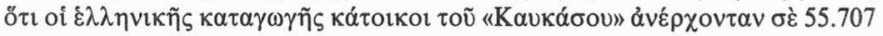

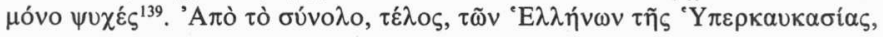

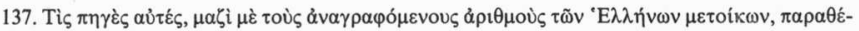

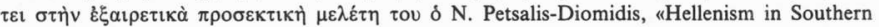
Russia and the Ukrainian Campaign: Their Effect on the Pontus Question (1919)", Balkan Studies

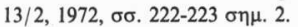

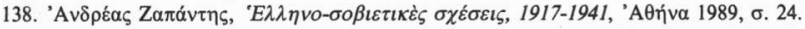

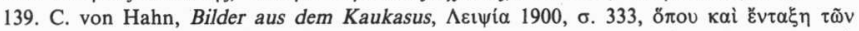

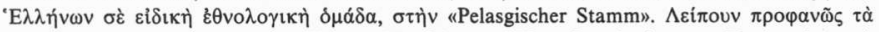

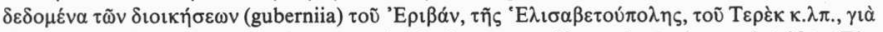

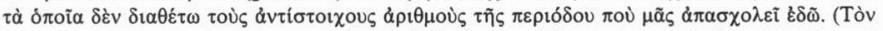

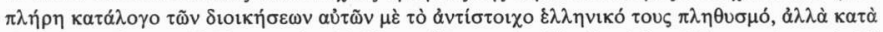

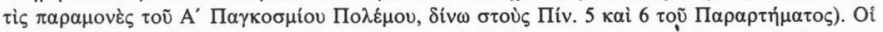

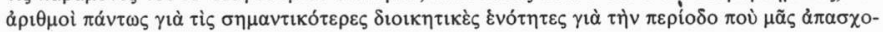




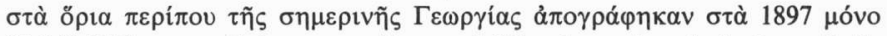

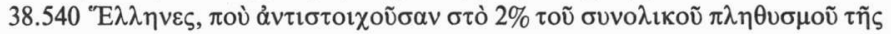

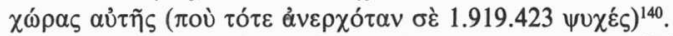

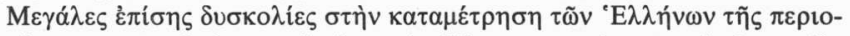

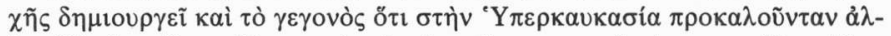

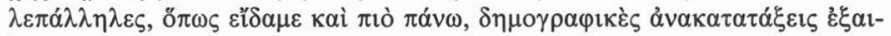

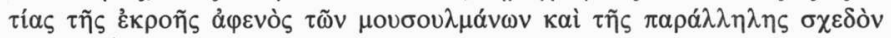

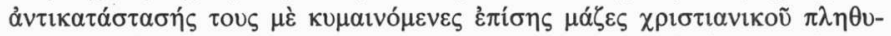

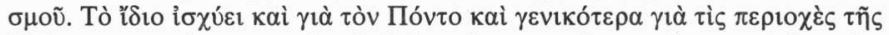

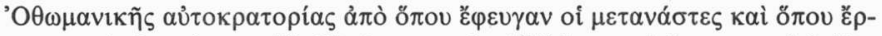

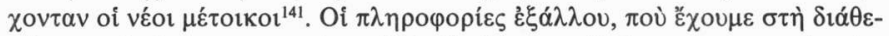

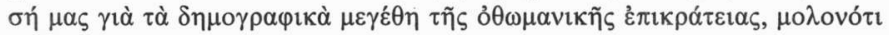

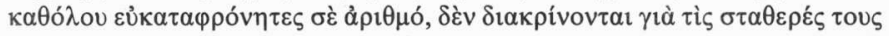

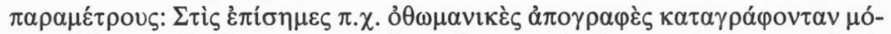

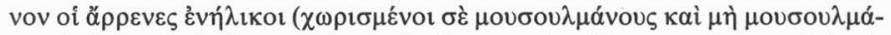

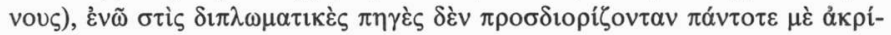

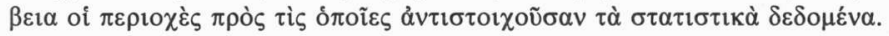

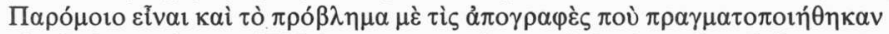

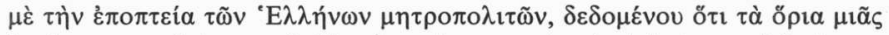

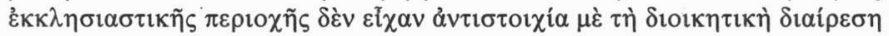

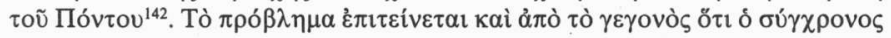

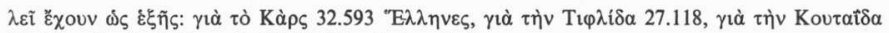

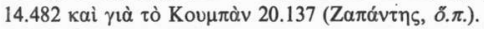

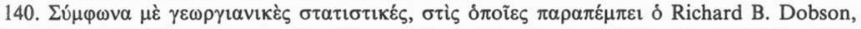

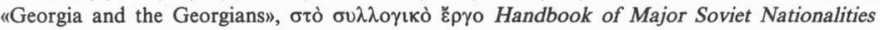
(

141. 'O Karpat, Ottoman Population, ๔. 11, ல்

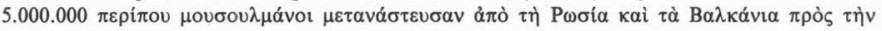

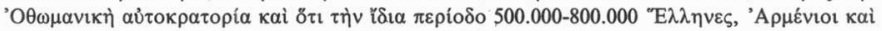

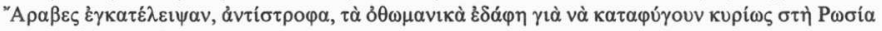

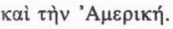

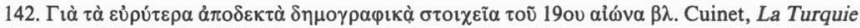

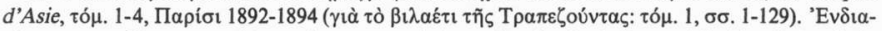

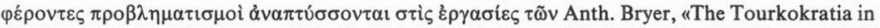
the Pontos: Some Problems and Preliminary Conclusions», Neo-Hellenika 1, 1970, бo. 30-54

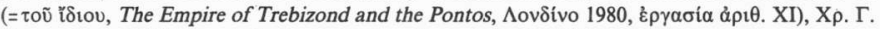

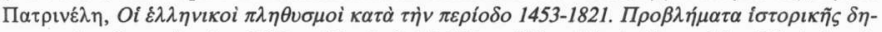

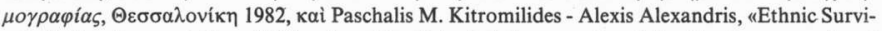
val, Nationalism and Forced Migration. The historical demography of the Greek community of

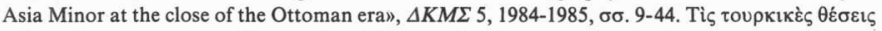

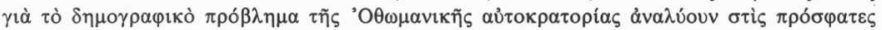

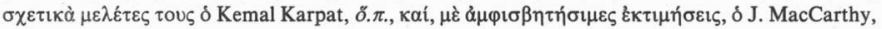
Muslims and Minorities. The Population of Ottoman Anatolia and the End of the Empire, N.

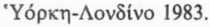




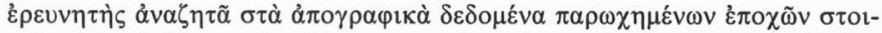

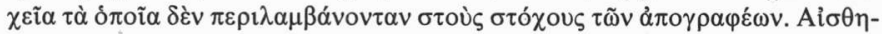

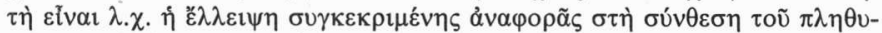

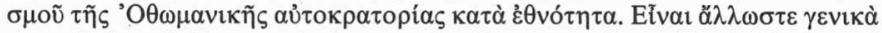

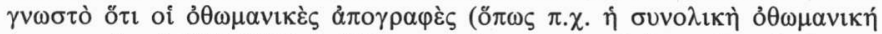

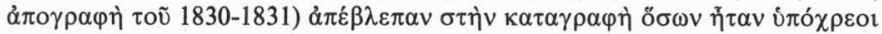

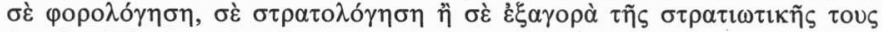

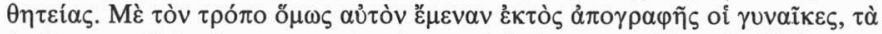

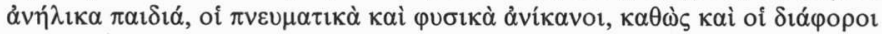

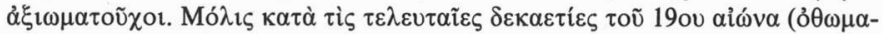

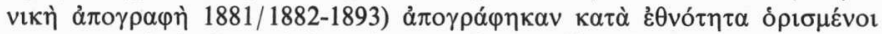

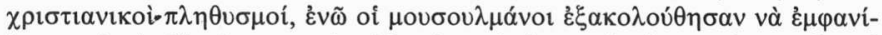

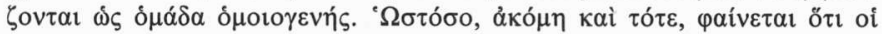

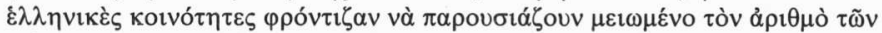

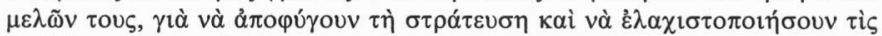

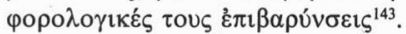

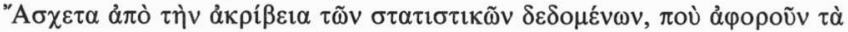

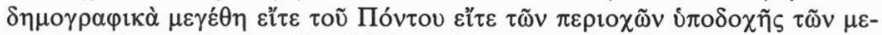

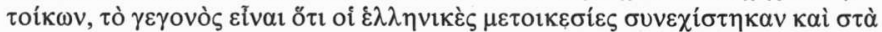

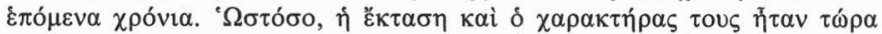

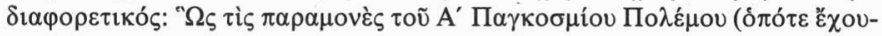

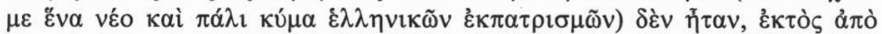

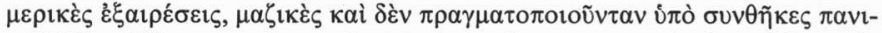

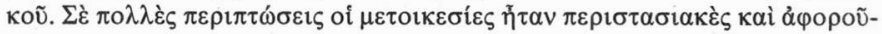

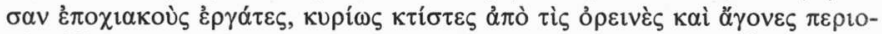

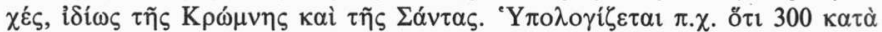

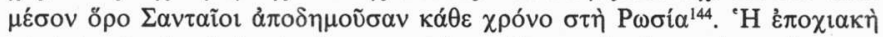

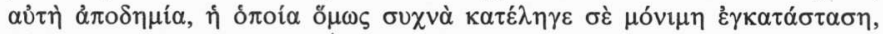

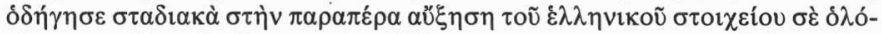

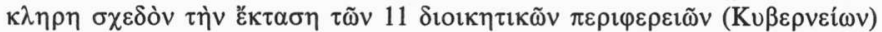

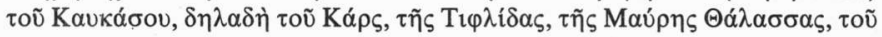

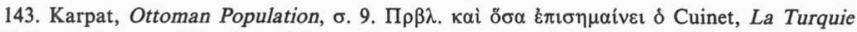

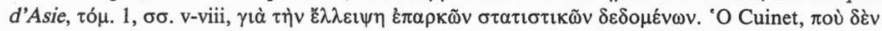

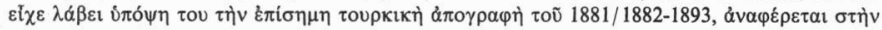

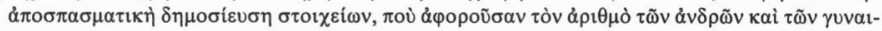

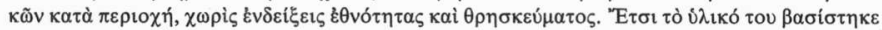

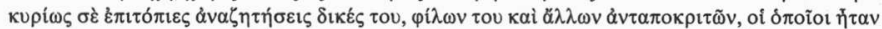

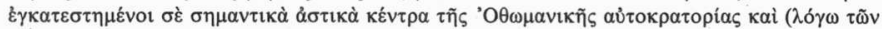

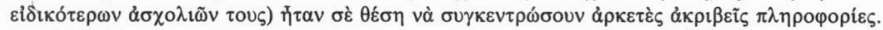

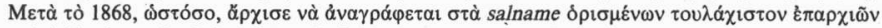

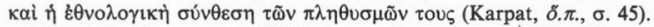

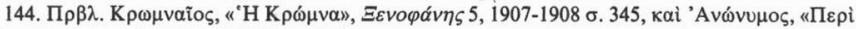

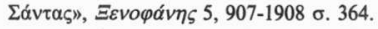




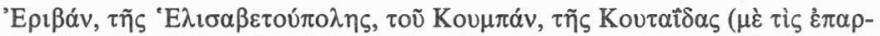

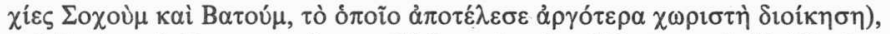

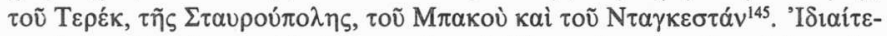

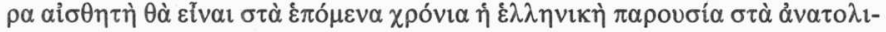

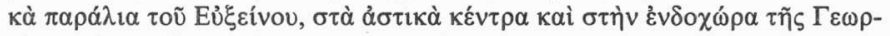

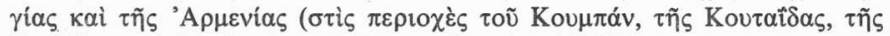

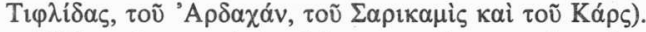

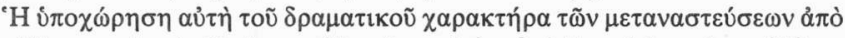

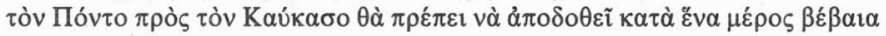

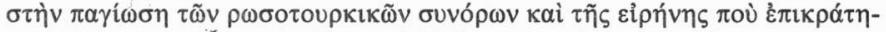

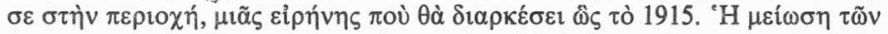

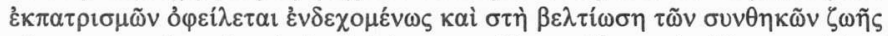

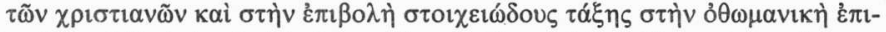

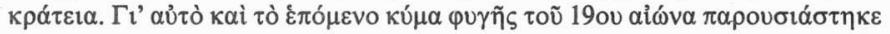

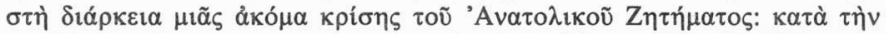

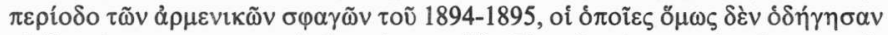

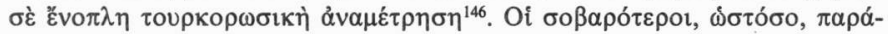

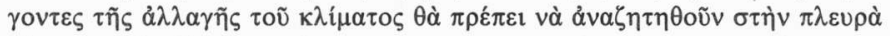

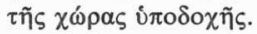

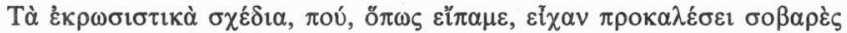

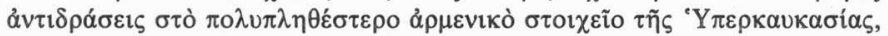

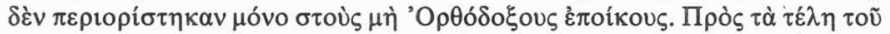

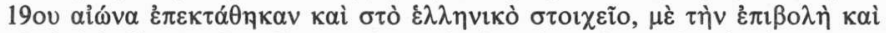

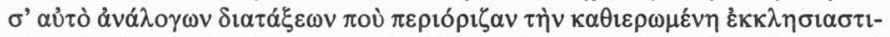

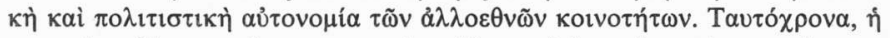

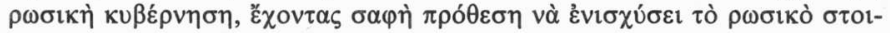

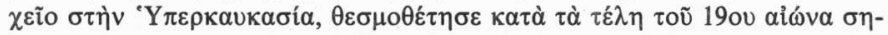

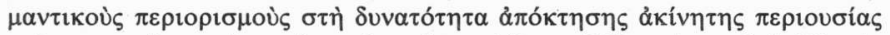

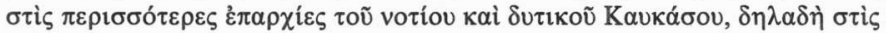

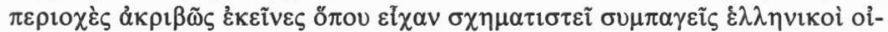
$\kappa \iota \sigma \mu \operatorname{ó}^{147}$.

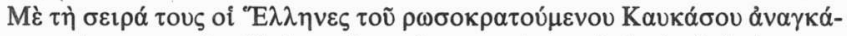

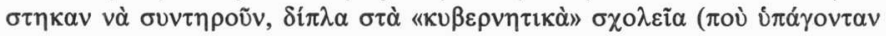

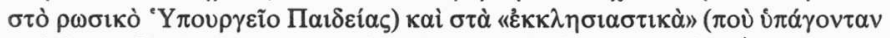

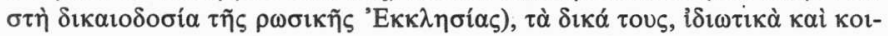

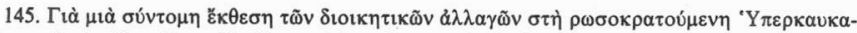

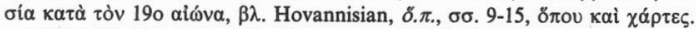

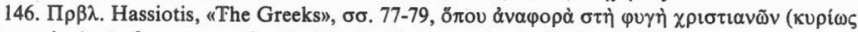

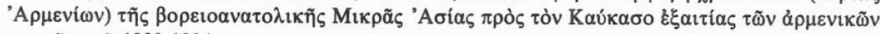

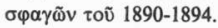

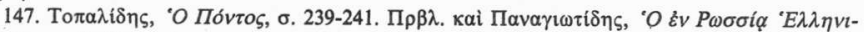

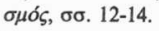




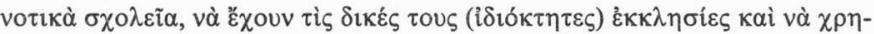

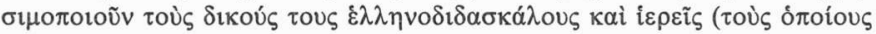

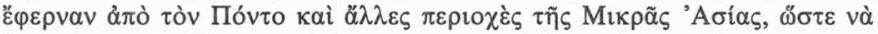

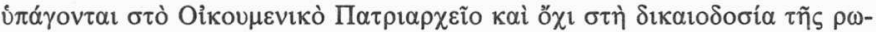

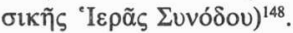

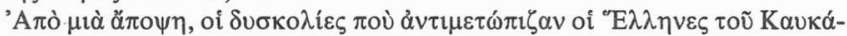

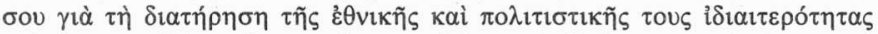

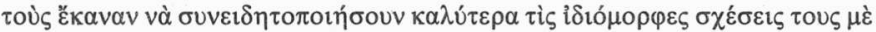

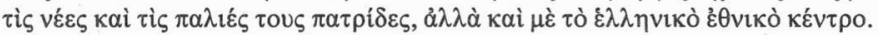

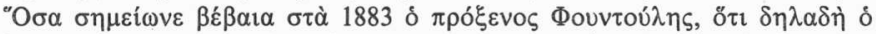

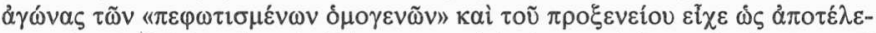

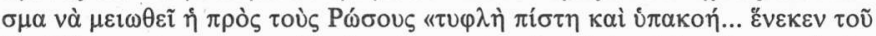

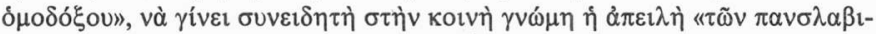

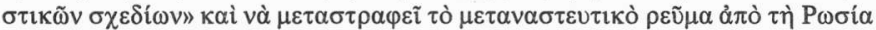

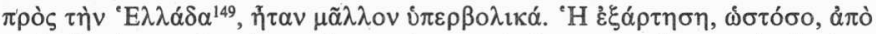

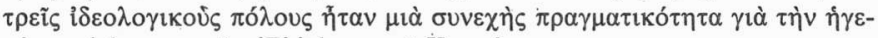

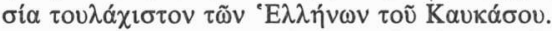

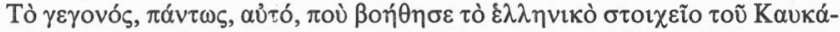

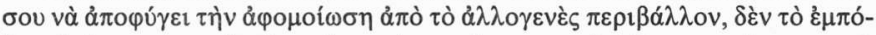

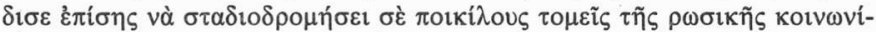

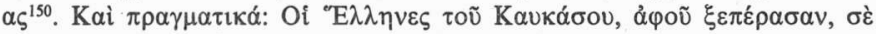

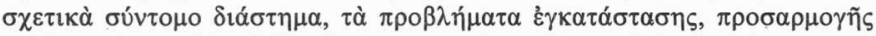

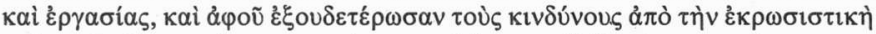

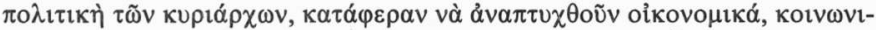

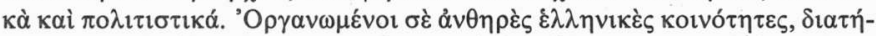

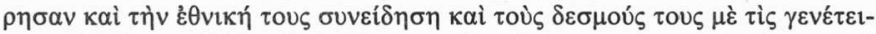

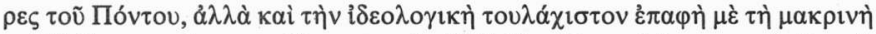

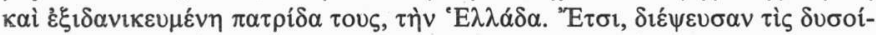

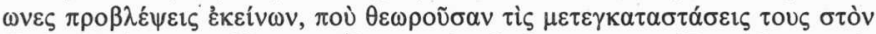

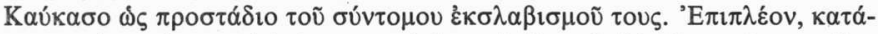

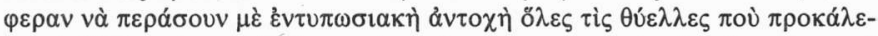

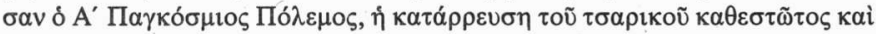

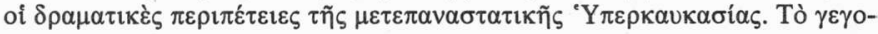

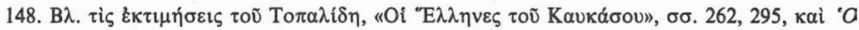

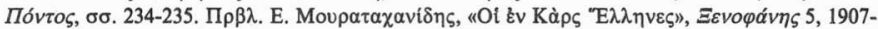

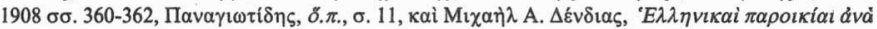

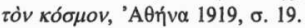

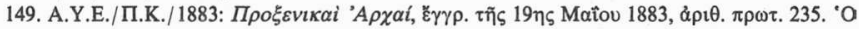

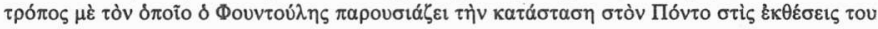

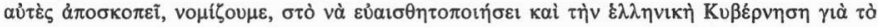
$\pi \rho \delta ́ \beta \lambda \eta \mu \alpha$.

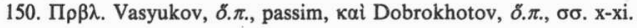


vò

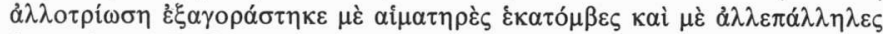

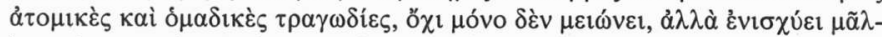

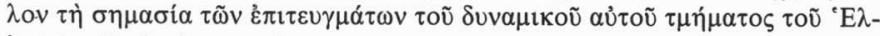

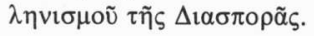




\section{EПIMETPO

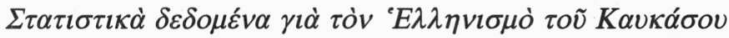

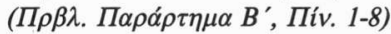

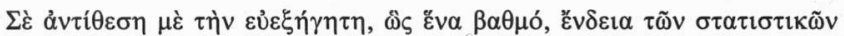

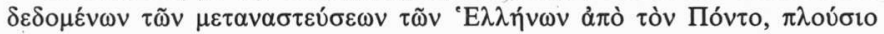

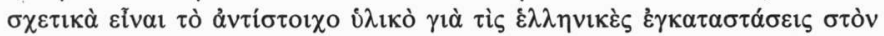

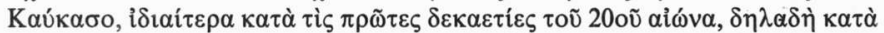

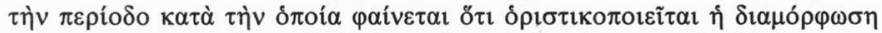

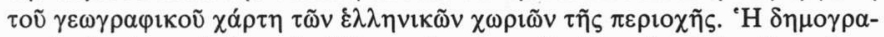

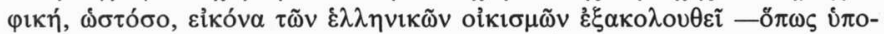

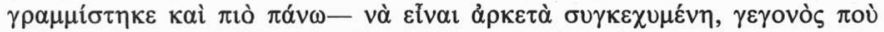

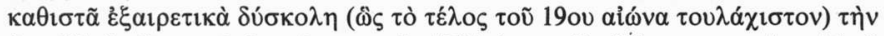

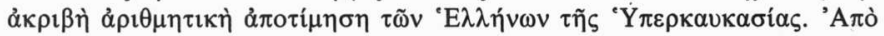

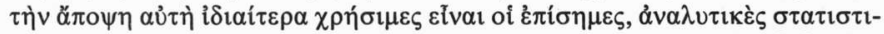

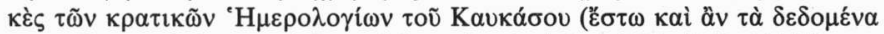

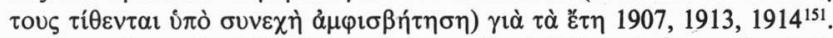

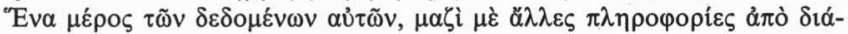

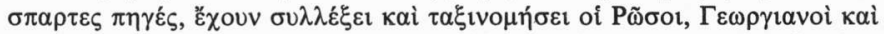

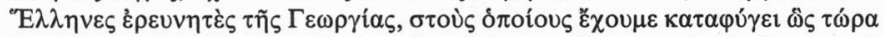

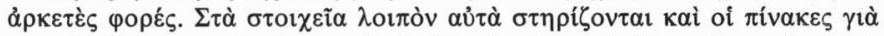

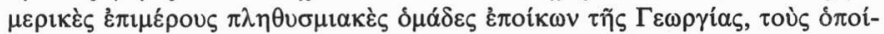

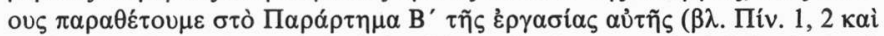

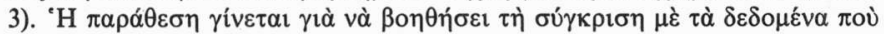

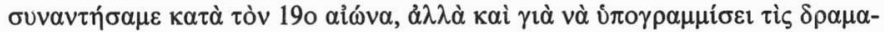

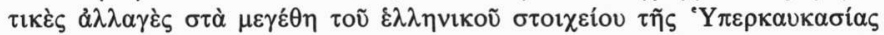

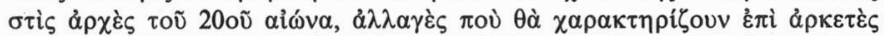

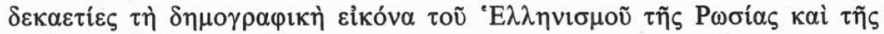

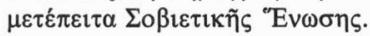

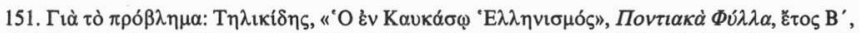

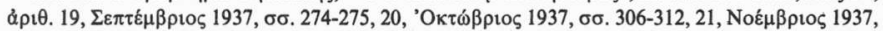

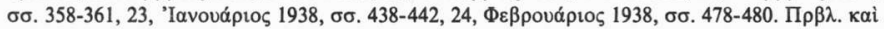

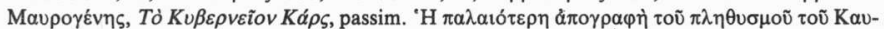

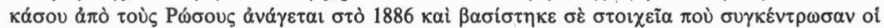

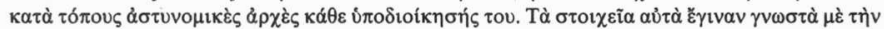

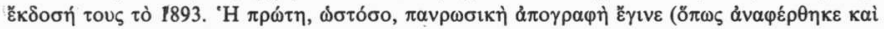

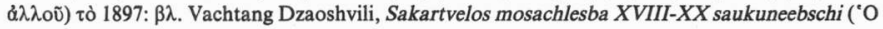

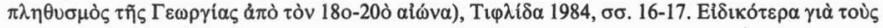

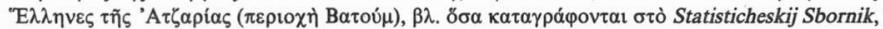

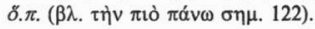




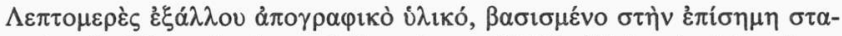

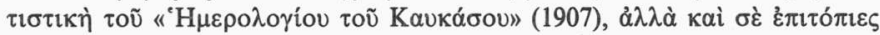

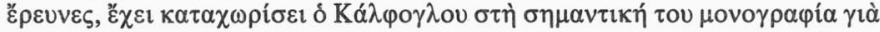

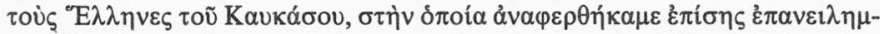

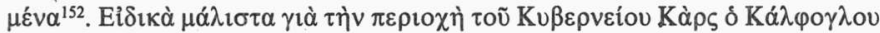

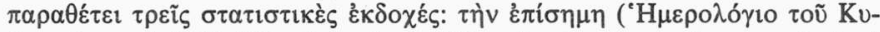

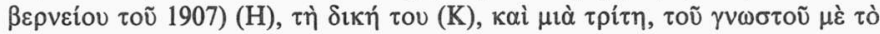

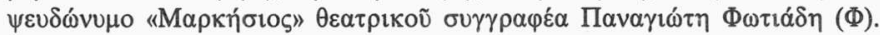

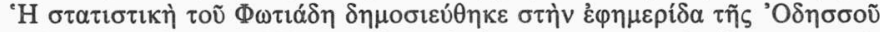

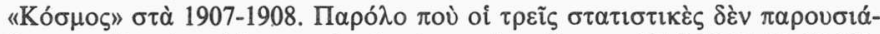

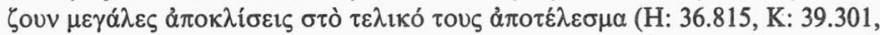

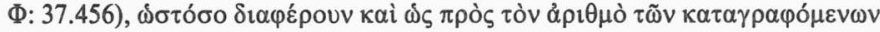

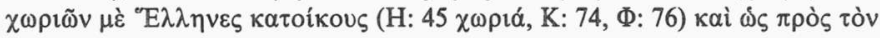

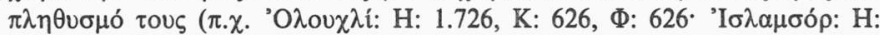
$1.201, \mathrm{~K}: 626, \Phi: 406)$.

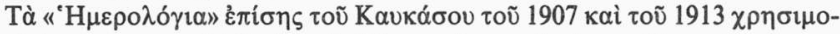

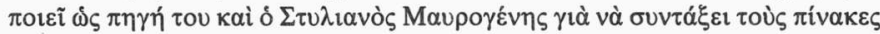

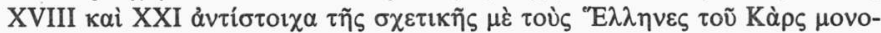

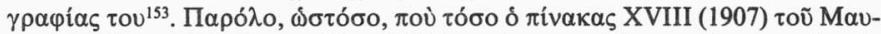

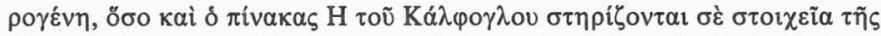

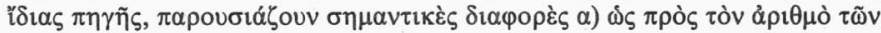

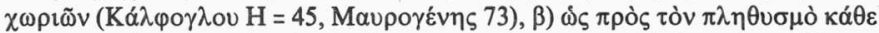

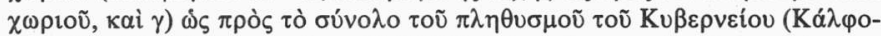

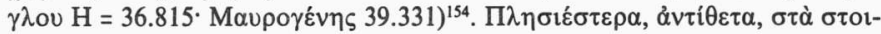

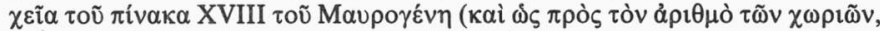

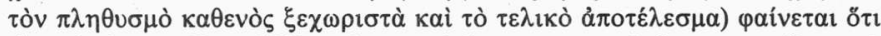

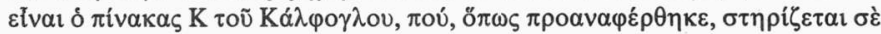

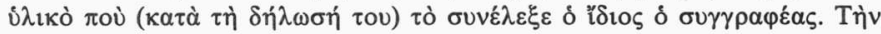

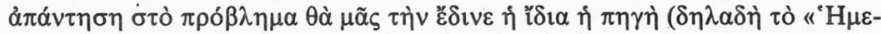

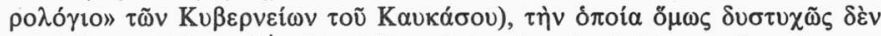

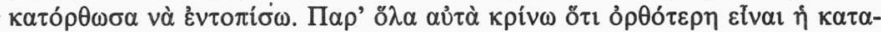

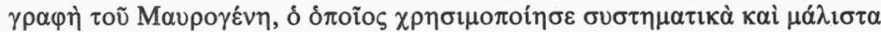

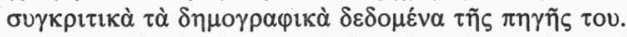

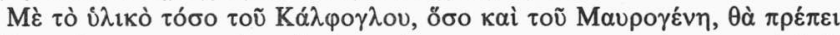

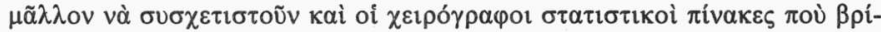

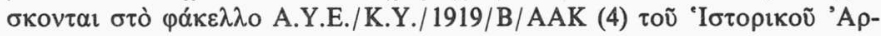

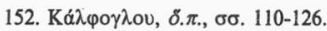

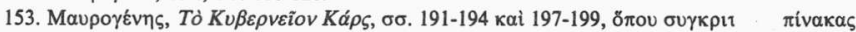

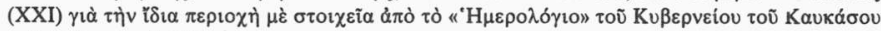

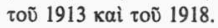

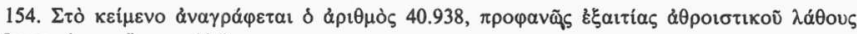

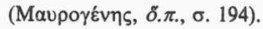




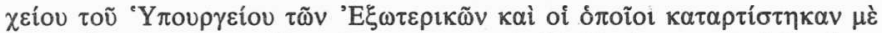

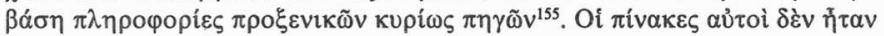

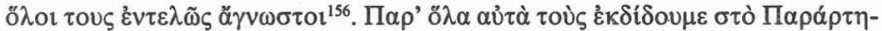

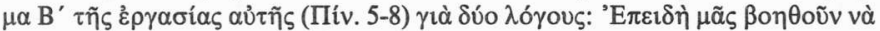

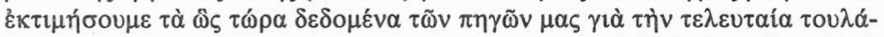

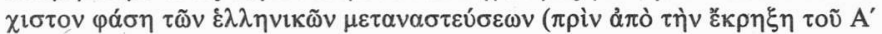

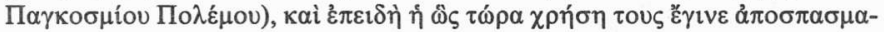

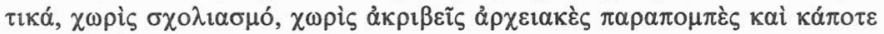

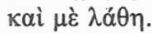

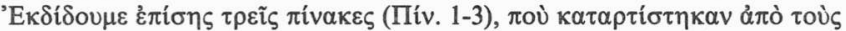

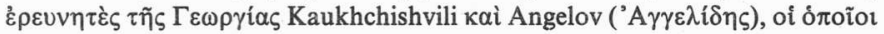

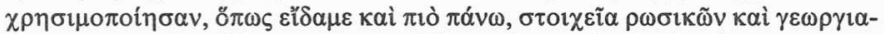

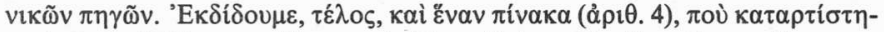

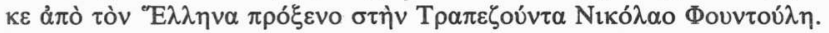

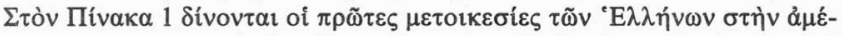

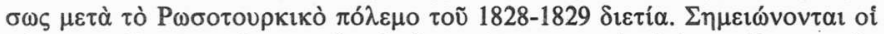

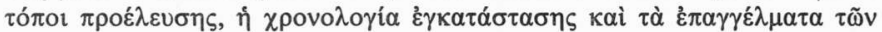

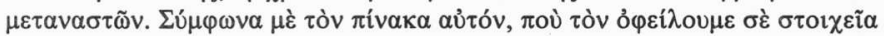

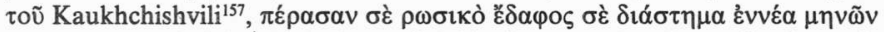

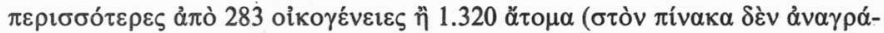

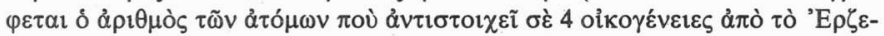

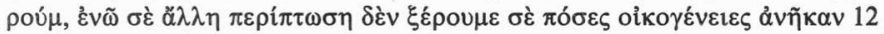

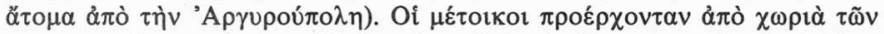

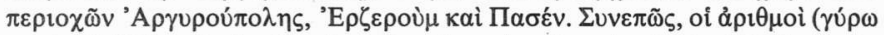

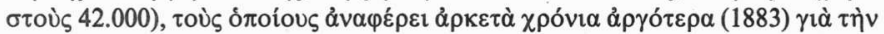

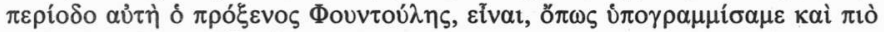

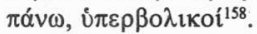

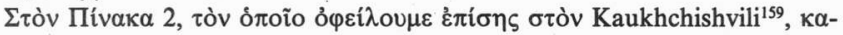

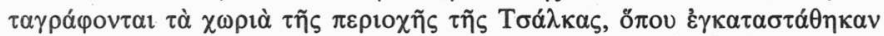

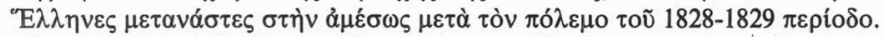

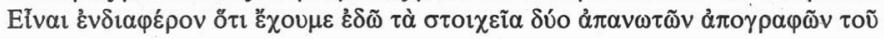

155. А.Y.Е./П.К./1919/B/AAK (4).

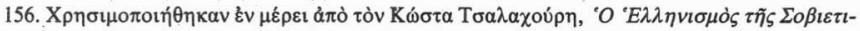

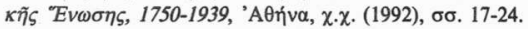

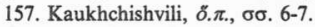

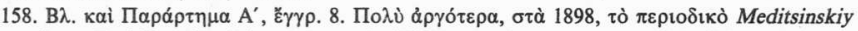

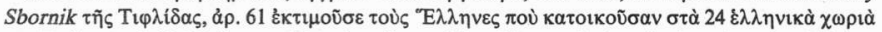

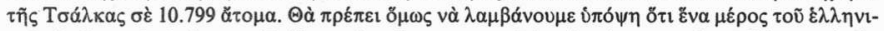

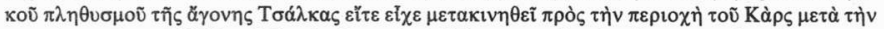

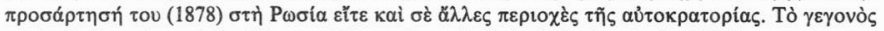

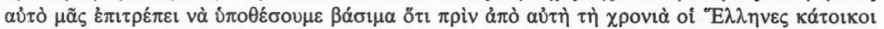

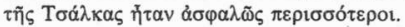

159. Kaukhchishvili, ס̊.л., б. 9. 


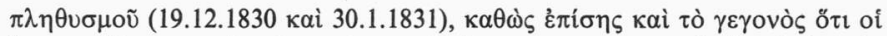

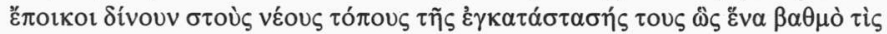

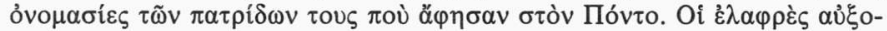

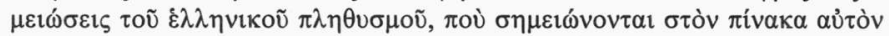

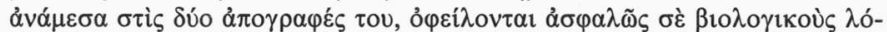

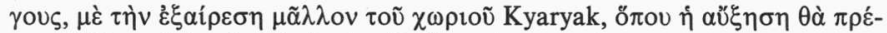

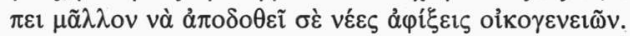

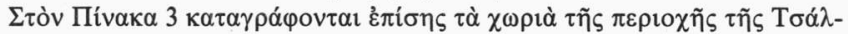

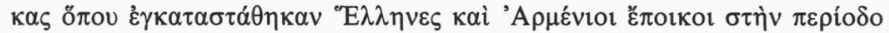

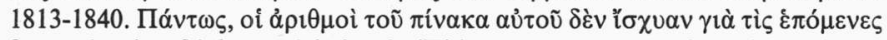

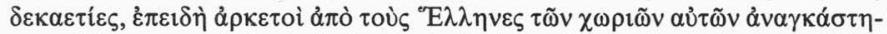

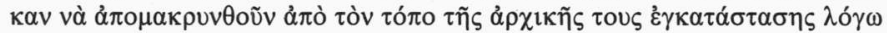

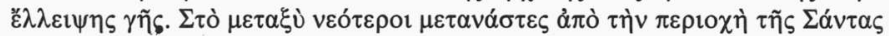

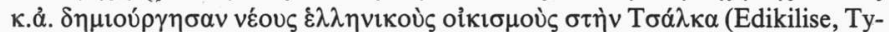
ak-Kilise, Menevik, Livad, Sanda, Imerà, Karakom, Saparkharaba к.ă.).

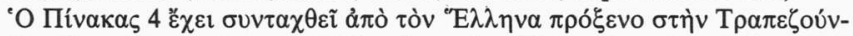

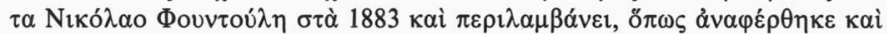

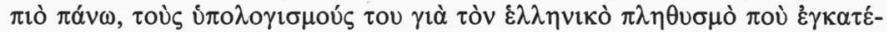

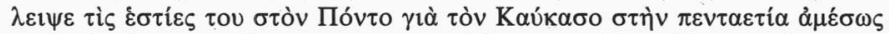

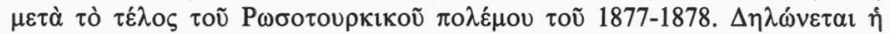

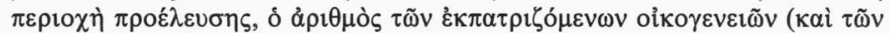

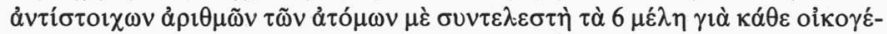

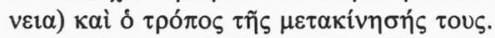

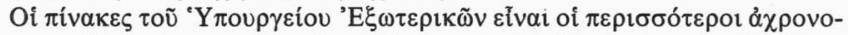

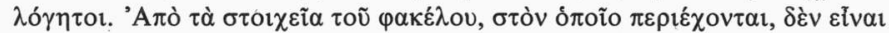

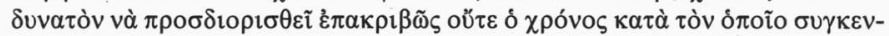

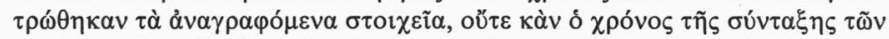

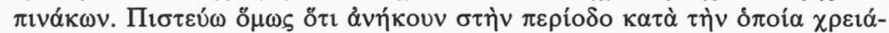

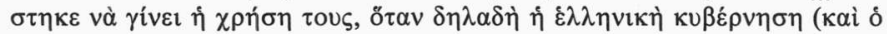

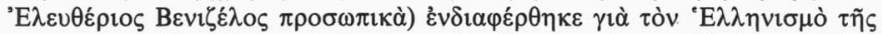

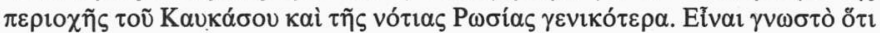

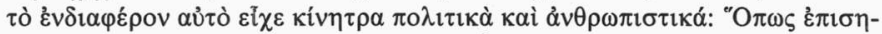

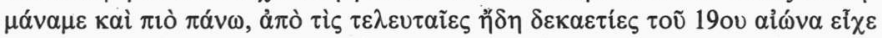

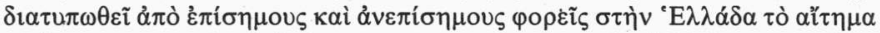

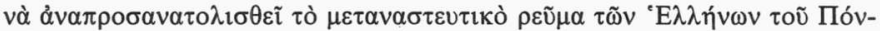

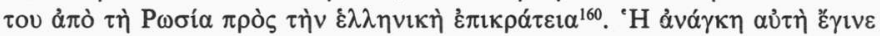

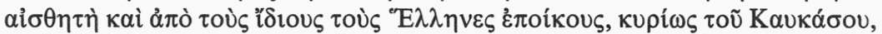

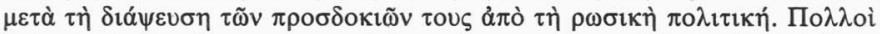

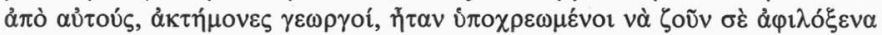

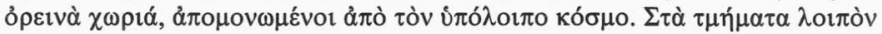

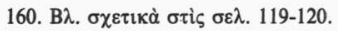




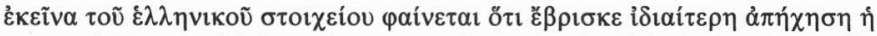

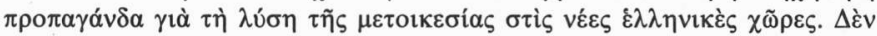

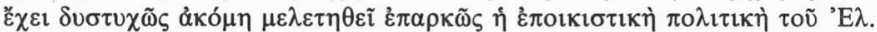

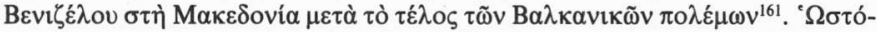

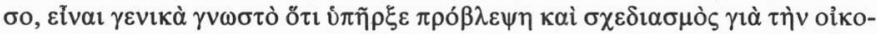

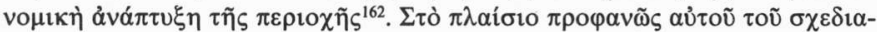

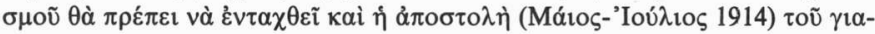

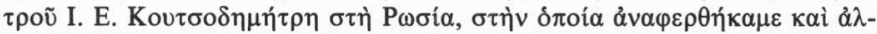
$\lambda 0^{163}$. 'H ảं

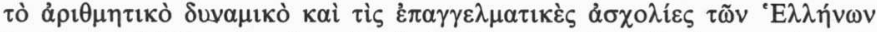

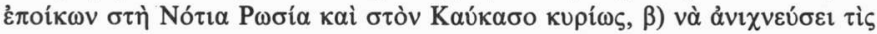
$\delta i \alpha \theta \varepsilon ́ \sigma \varepsilon เ \varsigma$

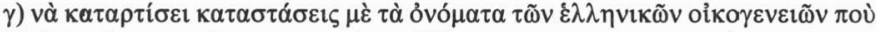

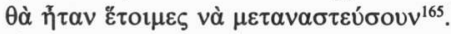

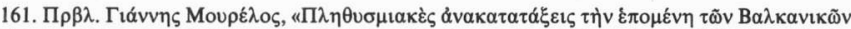

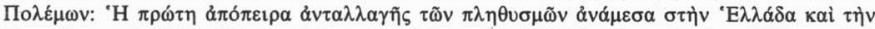

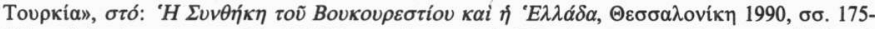

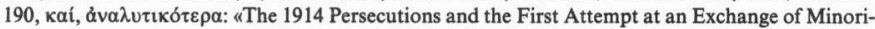
ties between Greece and Turkey", Balkan Studies 26, $1985 \sigma \sigma .389-413$, ס̈

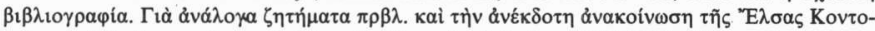

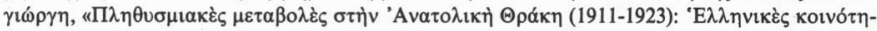

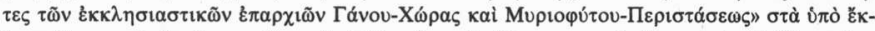

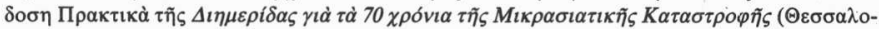

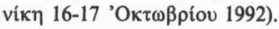

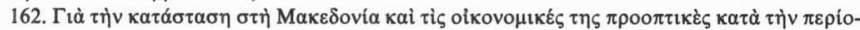

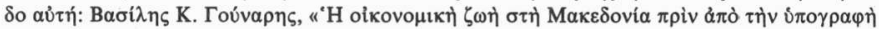

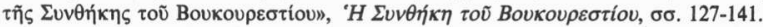

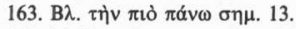

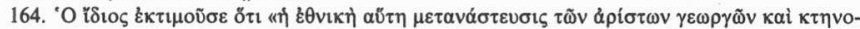

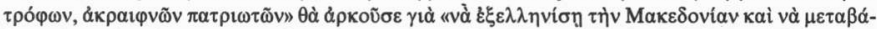

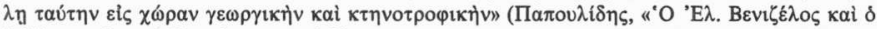

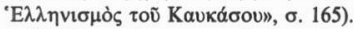

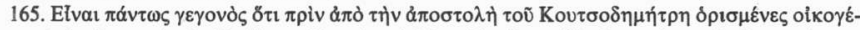

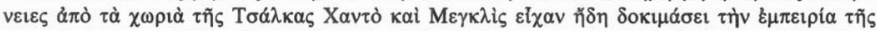

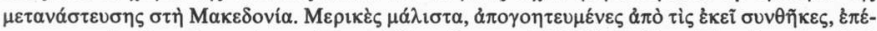

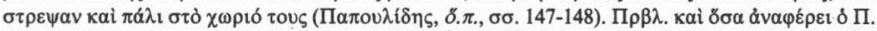

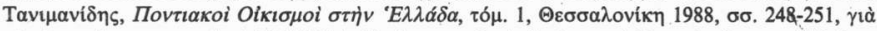

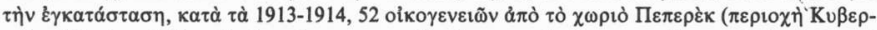

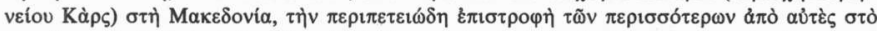

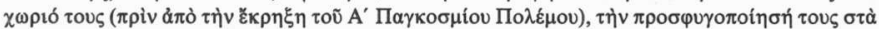

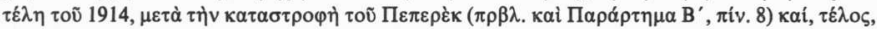

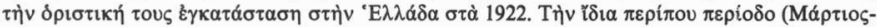

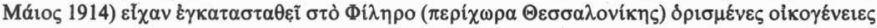

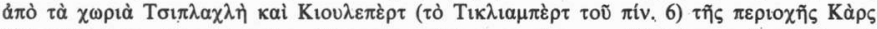

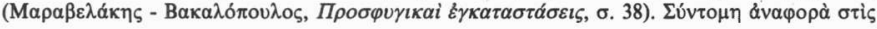




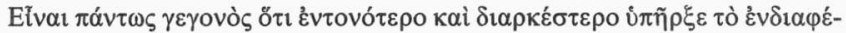

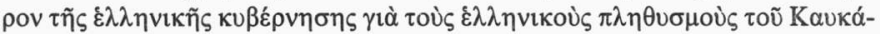

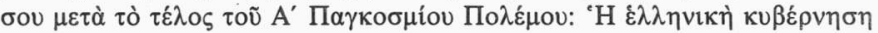

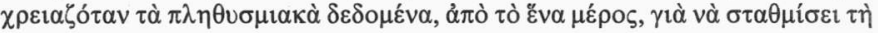

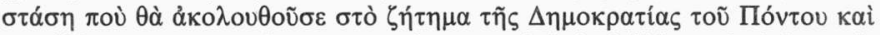

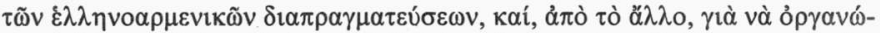

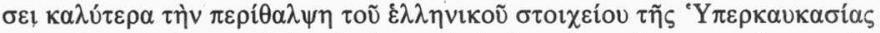

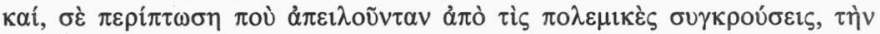

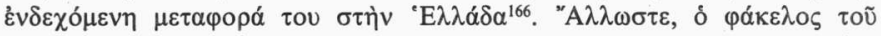

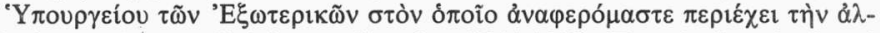

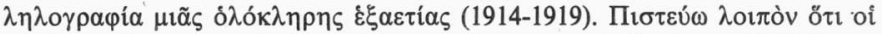

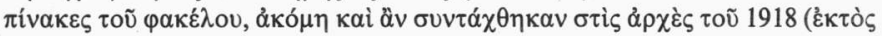

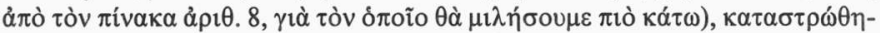

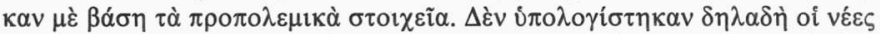

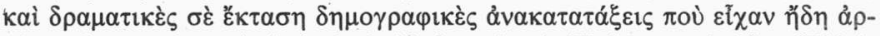

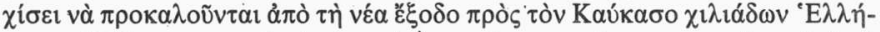

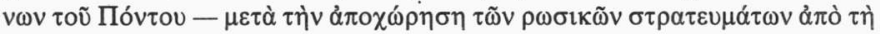

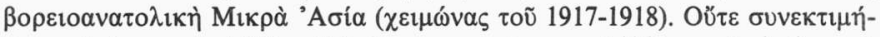

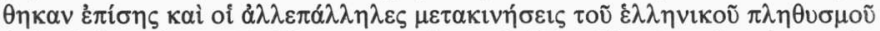

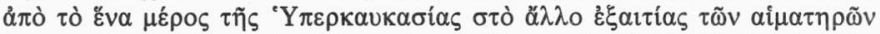

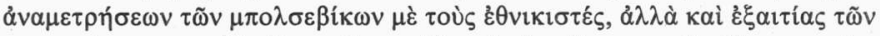

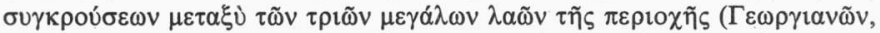

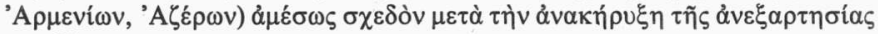
$\tau \operatorname{\tau ov}^{167}$.

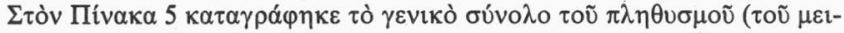

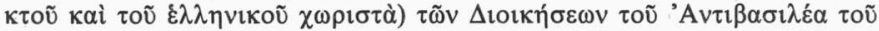

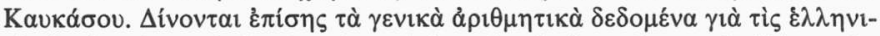

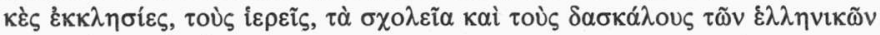

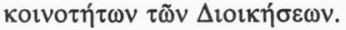

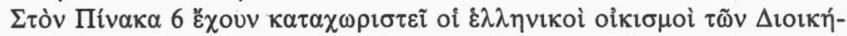

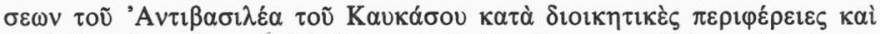

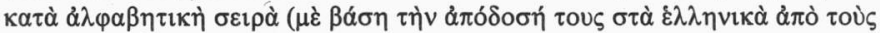

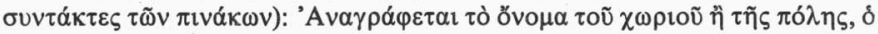

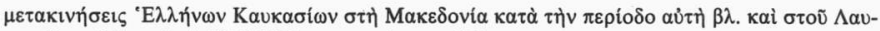

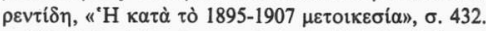

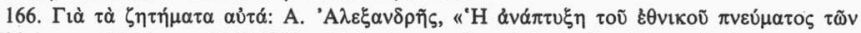

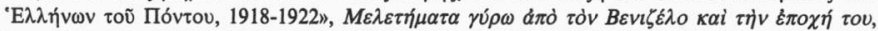

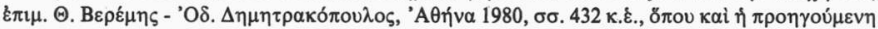

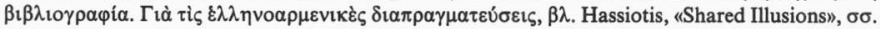

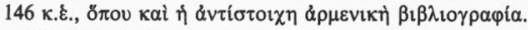

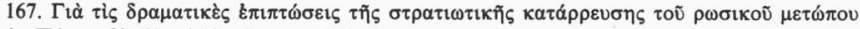

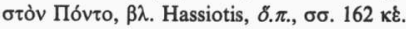




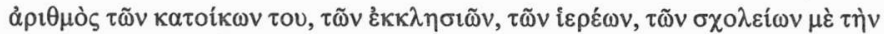

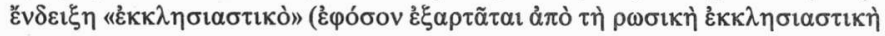

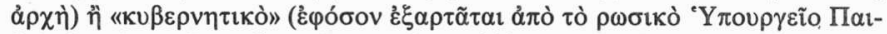

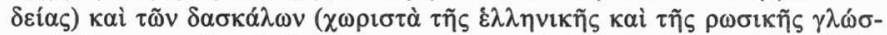

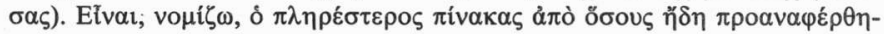

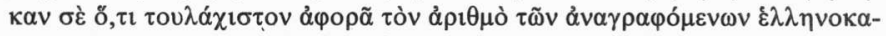

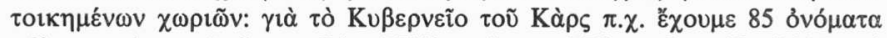

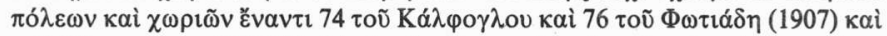

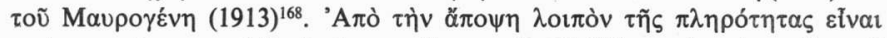

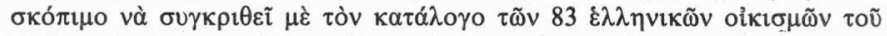

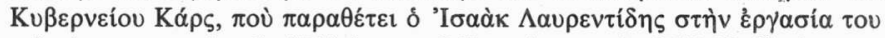

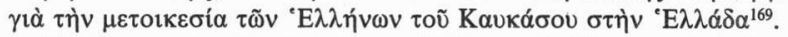

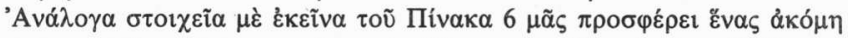

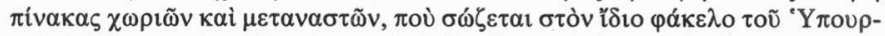

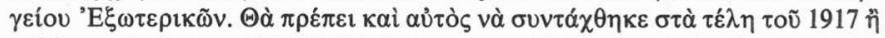

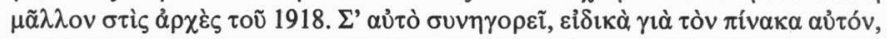

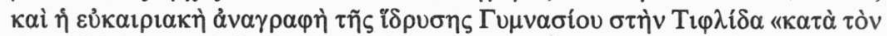

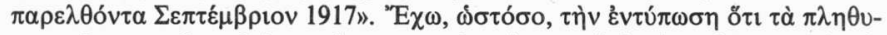

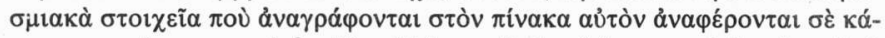

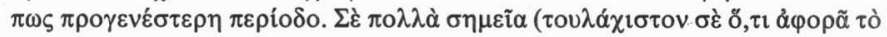

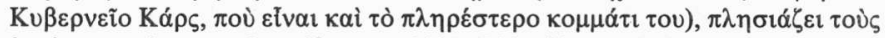

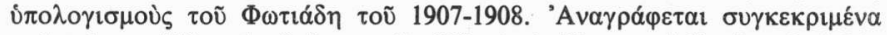

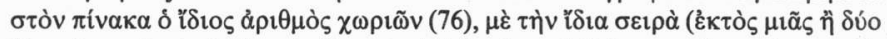

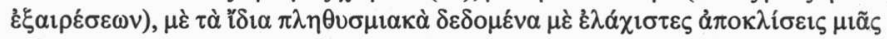

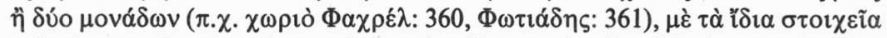

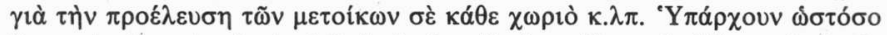

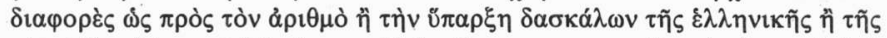

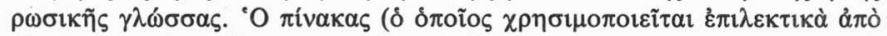

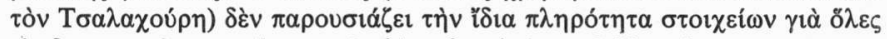

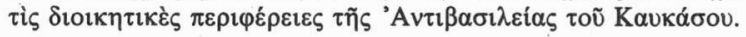

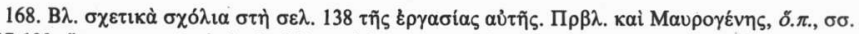

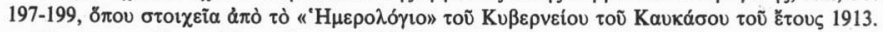

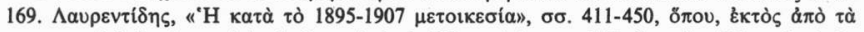

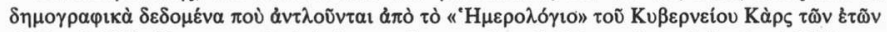

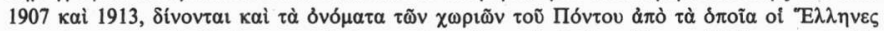

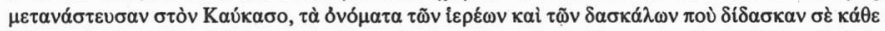

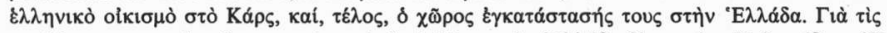

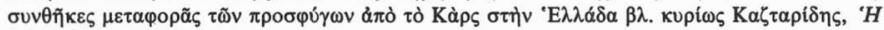

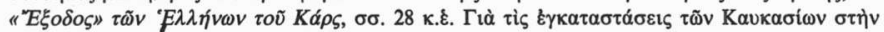

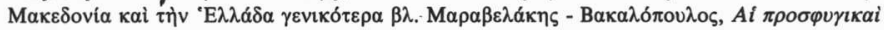

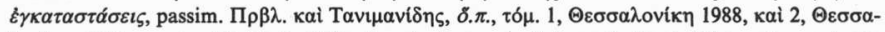

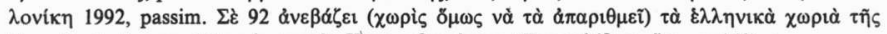

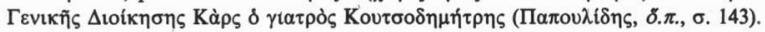




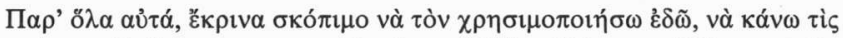

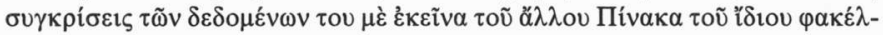

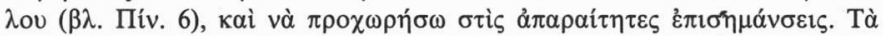

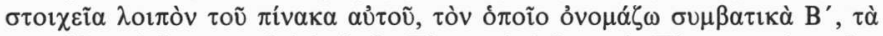

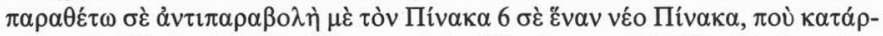

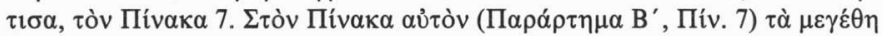

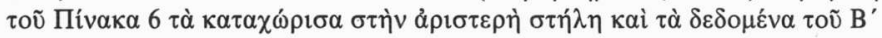

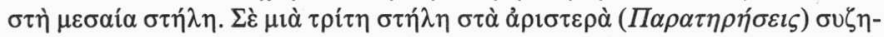

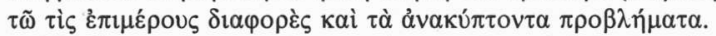

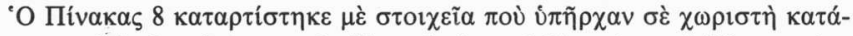

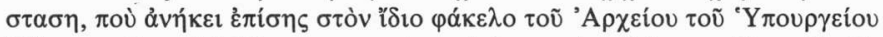

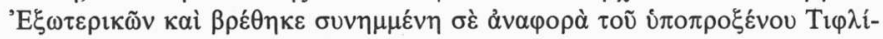

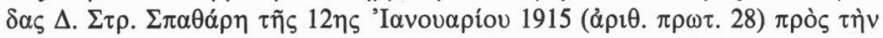

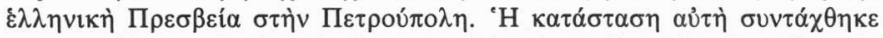

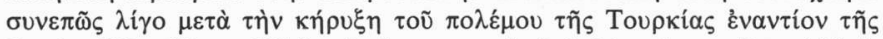

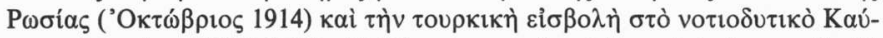

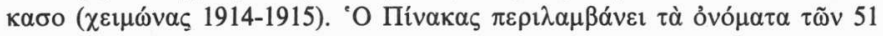

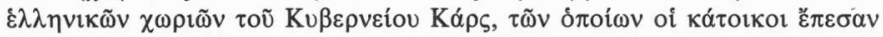

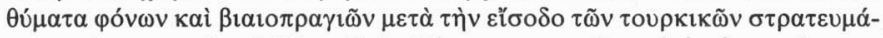

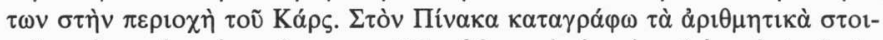

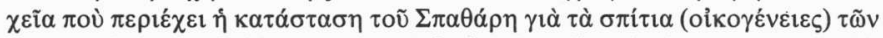

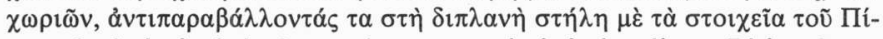

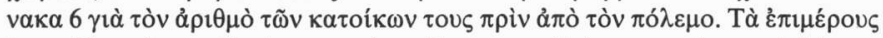

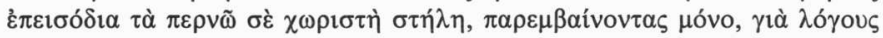

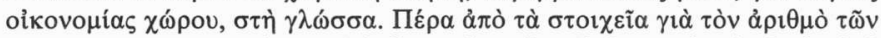

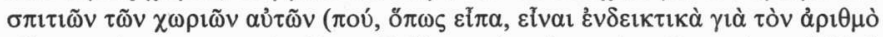

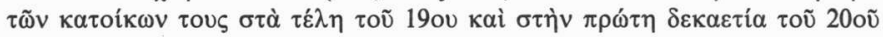

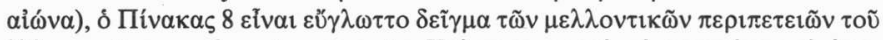

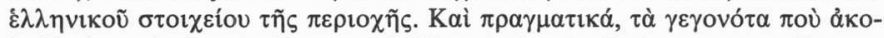

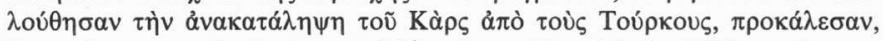

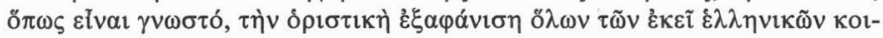

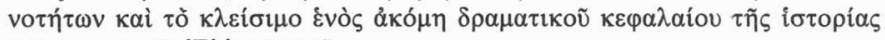

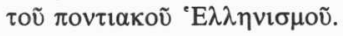




\title{
ПАРАРТНMА А'
}

\author{
ЕГГРАФА
}

1

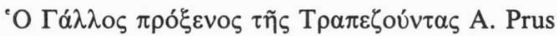

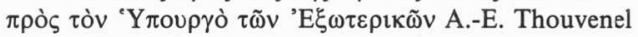

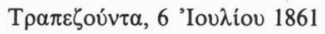

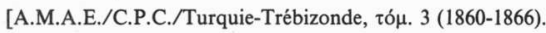

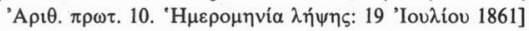

\section{Monsieur le Ministre,}

La nouvelle de la mort du Sultan et de l'avènement de Sa Majesté Abd-ulAziz, transmise par le télégraphe électrique à Sivas, et de là par des courriers aux principales villes du littoral et de l'ntérieur, a été annoncée le 30 juin, au matin, à Trébizonde par les crieus publics et par les saluts d'artillerie.

Ce double événement, à l'occasion duquel le corps consulaire est allé complimenter le Gouverneur Général Djemal Pacha, a vivement impressionné toutes les classes de la population et surtout de la population musulmane. Cette dernière, qui n'a pas moins eu à souffrir que les rayas des fautes du règne qui vient de finir, en parle sans aucun ménagement et voit avec la satisfaction la plus entière le pouvoir passer entre les mains d'un souverain, que ses premiers actes ont déjà rendu populaire, et dont l'énergie personnelle et les opinions religieuses bien connues, lui promettent tout au moins, dit-elle, un gouverneur nationals.

L'ordre n'a pas été un seul instant troublé à Trébizonde ni aux environs, mais à Samsoun une bande de Turcs fanatiques, réunis dans les cafés, a montré à l'égard des Chrétiens des dispositions assez inquiétantes, pour que le gouverneur Kiamil-Pacha envoyât ses cavas les disperser et défendre tout rassemblement sous peine d'arrestation.

De pareils faits, Monsieur le Ministre, sont d'autant plus regrettables, que des centaines de familles grecques et arméniennes de ces contrées, lasses d'un joug intolérable, sont à la veille d'émigrer en Crimée, où le gouvernement russe songe sérieusement à repeupler le sol abandonné par les Tartares.

D'après des informations que j'ai lieu de croire exactes, les conditions arrêtées récemment entre les autorités de la Crimée et une députation des Grecs d'Ordou seraient des plus encourageantes. Chaque émigrant, quel que soit son âge et son sexe, recevra 5 roubles d'argent (20 francs) en débarquant du bâtiment russe, qui doit venir le prendre, et vigt cinq kilogrammes de blé par mois pendant la première année. Un cheval, une vache et deux' boeufs de labour 
seront donnés à toute famille de cinq personnes. Une exemption d'impôts pendant dix ans sera, en outre, accordée à tout individu faisant partie de cette émigration, qu'il faut attribuer aux exactions et aux avanies de tout genre dont les cultivateurs principalement sont victimes. C'est ainsi qu'à Ordou et à Kérassunde, après avoir reçu, il y a vingt cinq ans, des terres de l'État, qu'ils ont défrichées et mises en valeur, sous la promesse qu'ils en deviendraient ensuite propriétaires, à la charge de payer les impôts, de pauvres paysans Grecs se sont vus forcés de payer des taxes arbitraires et exorbitantes, pour éviter une expulsion immédiate.

Il est grand temps que l'initiative du Souverain apporte un remède à un état de choses que menace de convertir en un vaste désert une des plus belles et des plus fertiles provinces de l'Empire.

Agréer l'hommage du respect avec lequel j'ai l'honneur d'être, Monsieur le Ministre,

de Votre Excellence

Le très humble et très obéissant Serviteur

A. Prus

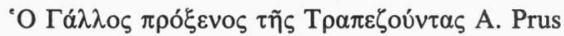

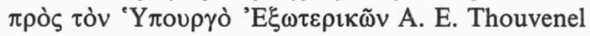

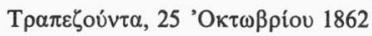

[A.M.A.E./C.P.C./Turquie-Trébizonde, $\tau$

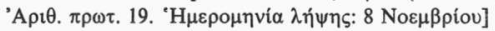

Monsieur le Ministre,

Le Vali de cet eyalet vient de faire une excursion de trois semaines dans l'intérieur de la province jusqu'à Gumuch-Khané, à une distance de vingtquatre heures de caravane de Trébizonde. Le motif avoué de ce voyage était l'examen de viser des obstacles qu'offre la construction toujours différée de la grande route de la Perse, mais, d'après une source digne de foi, son but réel était d'arrêter par tous les moyens possibles, au besoin par la force, le flot des émigrants Grecs et Arméniens, qui vont combler en Géorgie et en Crimée les vides laissés par l'émigration tartare. Des milliers de familles ont ainsi quitté, depuis trois ans, le seul district de Gumuch-Khané, abandonnant leurs maisons et leurs cultures, dont le produit net ne suffisait pas à leurs premiers besoins. Ces malheureuses populations, découragées en Anatolie par le déplorable état des voies de communication, qui, en formant les débouchés, les prive du fruit de leur travail, exaspérées par la rapacité des agents du fisc, qui exigent la dime sur la botte d'herbe que de pauvres femmes rapportent des vaines pâtures des 
montagnes, sont irrésistiblement attirées vers la Russie par des concessions de terres, des distributions de vivres et de bétail et des exemptions d'impôts.

Monsieur Malsiniotis, Vice Consul de Grèce à Trébizonde, vient d'être appelé à Ismaïl et remplacé par Monsieur Vratzanos, Vice Consul hellène à Serès. J'ai lieu de croire que la Légation Russe à Athènes n'est pas étrangère à cette mutation, provoquée par mon collègue de Russie, qui n'avait pas trouvé en Monsieur Malsiniotis un instrument aussi docile que dans ses prédécesseurs.

Agréez l'hommage du respectance lequel j'ai l'honneur d'être Monsieur le Ministre, de Votre Excellence,

Le très humble et très obéissant Serviteur

A. Prus

3

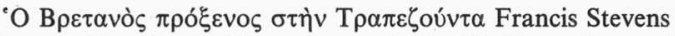

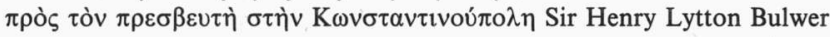

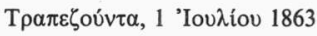

[P.R.O./F.O. 195/762 (1863)]

Sir,

As far back as July 1861, in my despatch no 13, I had the honour of bringing to Your Excellency's notice an intention then entertained by a number of Christian families, chiefly Greeks, to emigrate to Russia. The Porte did not allow them to carry out their plans. Recently the Russian Consul, who has never ceased to encourage the Christians of this coast to change their nationality, has revived the question relating to the Christians of Ordoo and Lazistan and, within the last few weeks, obtained viziriel letter to the pasha authorizing the emigration under special conditions among and other the sale of the landed property of the emigrants, of some four hundred families but His Excellency has refused to execute the Vizir's orders, because the Consul will not admit of the terms laid down by the Porte, which fact has produced a sharp discussion between the pasha and the Consul.

The latter I learn is on the eve of proceeding to Constantinople to engage the Russian Ministry to cause the Porte to enforce the viziriel orders above stated. If the Consul gains the case, and the families are allowed to emigrate, other would be ame to follow the example to the detriment of the Turkish government.

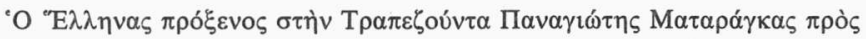




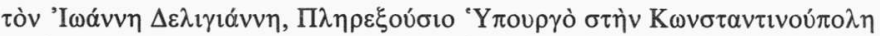

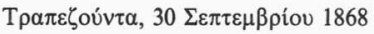

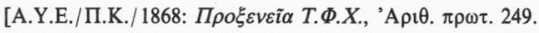

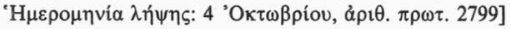

'E

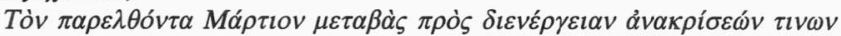

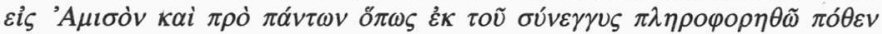

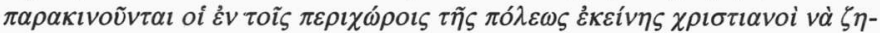

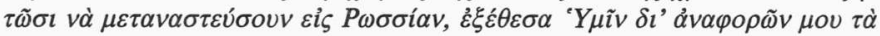
$\sigma v \mu \beta a ́ v \tau \alpha$.

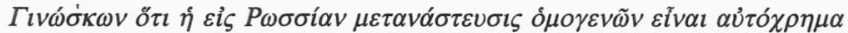

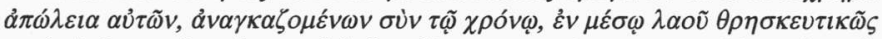

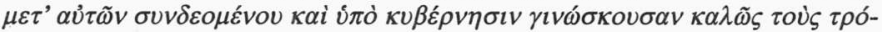

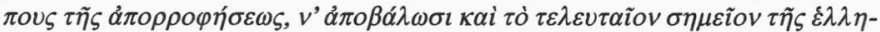

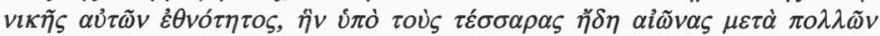

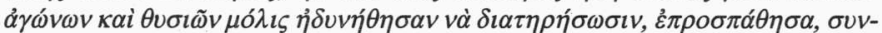

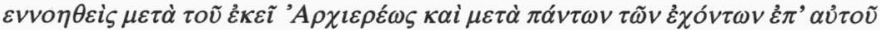

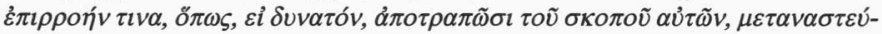

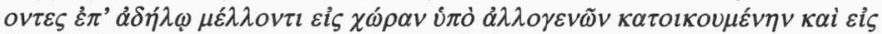

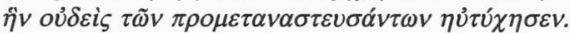

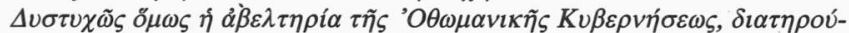

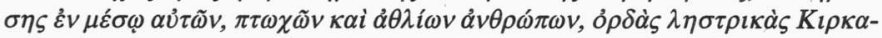

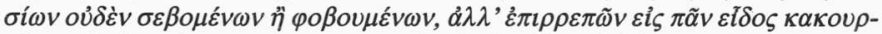

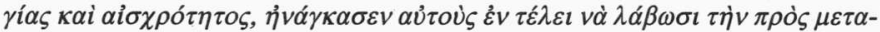

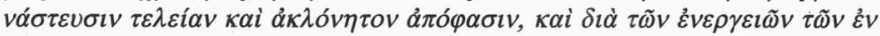

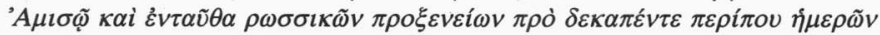

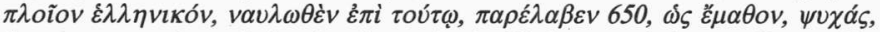

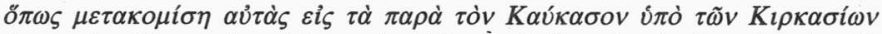

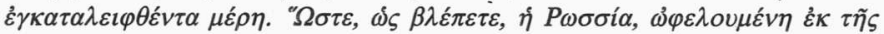

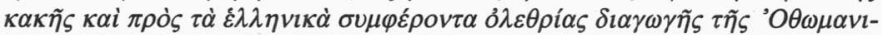

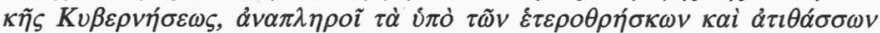

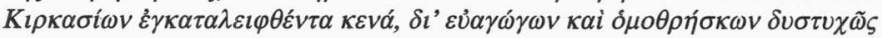

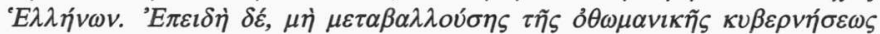

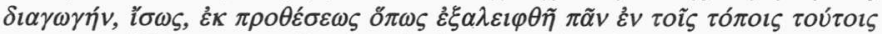

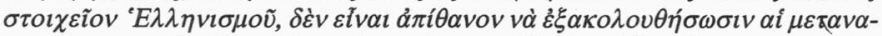

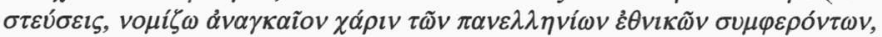

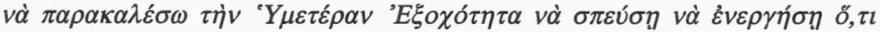

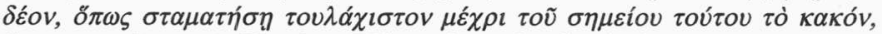

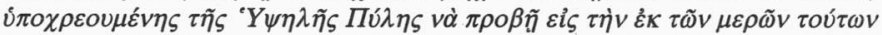

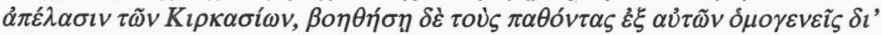

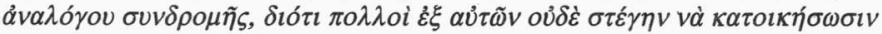




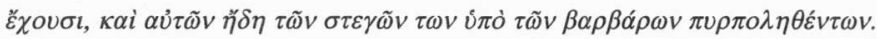

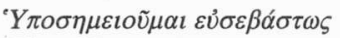

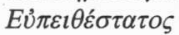

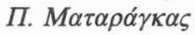

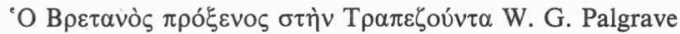

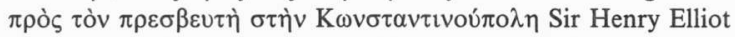

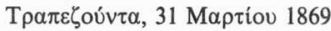

[P.R.O./F.O. 195/953 (1869-1872), ג $\rho \mathrm{l} \theta . \pi \rho \omega \tau .19]$

Sir,

It is my duty to report that a scheme for emigration from Turkey to Russia is now being actively carried rut among the inhabitants of this neighbourhood on a serious scale. The proposed emigrants are the so-called "Kroomlees", a race tenanting several villages to the East and South East of Trebizond. The "Kroomlees» thus denominated from the hamlet of "Kroom», their headquarters, are folk of Byzantine extraction. They professed mahometanism for upwards of three centuries and a half, but have within the last ten years availed themselves of the ever-growing Russian influence in these parts, to proclaim themselves Greek Christians. The motive they now assign for wishing to emigrate is the military conscription, which the Ottoman Government continues to enforce amongst them, in spite of their change of creed; the motor is the Russian Consul-General of Trebizond.

These "Kroomlees", who are from three to four thousand souls in number, have this week addressed a petition to all the Consulates, the British included, at Trebizond, requesting their joint support, and the Russian Consul-General has begged my co-operation in favour of these "persecuted Christians".

That the Kroomlees grievance is merely a pretext, and a very poor one, needs no explanation. But even were the reverse the case, the British Consulate would, it seems to me, have no good ground for interfering in an affair of purely internal Ottoman administration, either officially or officiously; unless of course under superior orders. Some of my colleagues will however, I am informed, act otherwise; indeed the Russian Consul-General has already done so; on the title that some of the "Kroomlees» are furnished with Russian passports; documents of easy purchase.

Indeed I should not have thought it right to trouble Your Excellency with a despatch on a topic of so little concern, had I not been expressly requested by the local government to communicate these facts; a request with which, after due delay, it seemed to me my duty to comply. 
I add on this occasion, and quoting as near as possible, the very words, some statements made me by the lacal government, as follows:

"There are two centres of Russian action for emigration and other purposes on this coast, namely Samsoon and Trebizond. But the Russian agent at the latter has better success; partly because he deals with a population of artisans, stone-cutters and the like, who, on emigration to Russia, find ready work and high wages; partly from his own rank of Consul-General, complied with about eight thousand roubles of Secret Service money per annum over and above his regular salary. Whereas he of Samsoon has a less enterprising race and poorer materials to work on; besides being himself merely a Consul, nor highly paid».

"The third centre of russianising action, namely Batoom, may be looked on as already virtually lost to Turkeyn.

Thus far my informants. Taking their information at its own value, and neither confirming nor impugning it, I respectfully subjoin.

That Russian action and intrigue, however, skilful and well supported, would have less effect, but for the fiscal system, the over-taxation and the other faults of Ottoman Provincial Administration, noted in the Appendix on that subject, which I had the honour of sending in with the general report last year, causes which nowise excuse disaffection and treason, but which unfailingly produce them, while they loosen the attachment of the subjects in general, especially the Christian, to their rulers and facilitate intrigue, perhaps prepare a crisis.

I have the honour to be, with profound respect, Your Excellency's most obedient, humble servant,

W. Gifford Palgrave

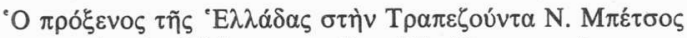

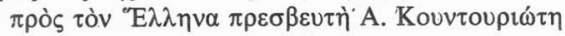

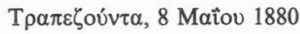

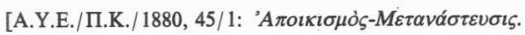

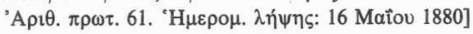

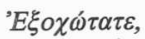

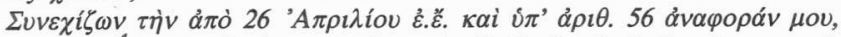

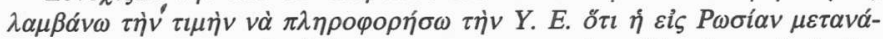

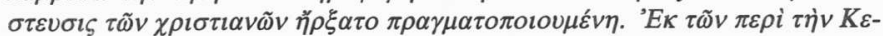

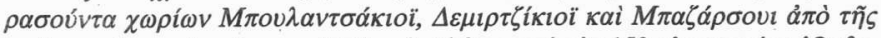

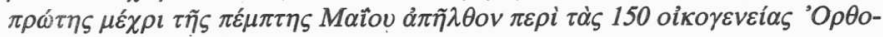




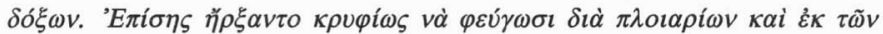

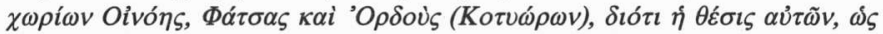

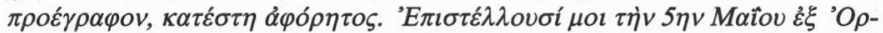

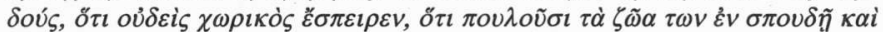

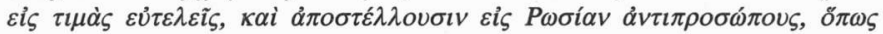

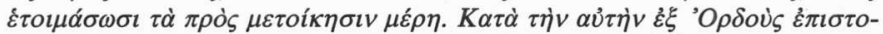

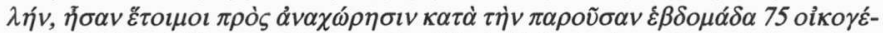

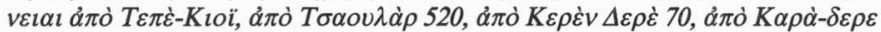

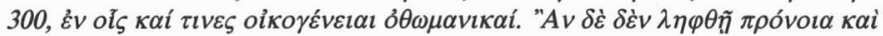

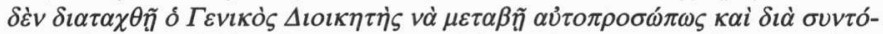

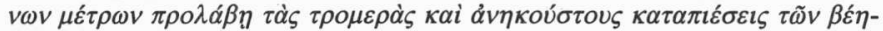

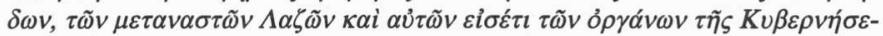

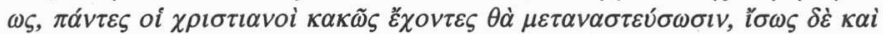

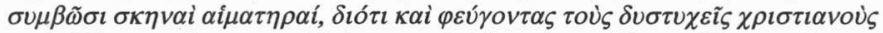

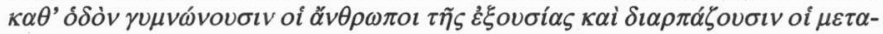

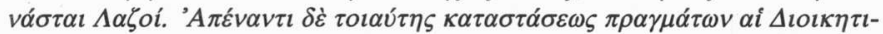

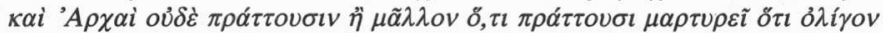

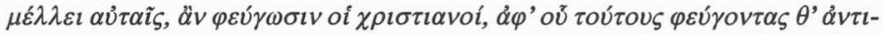

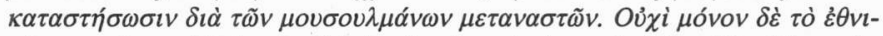

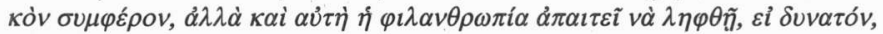

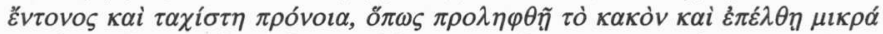

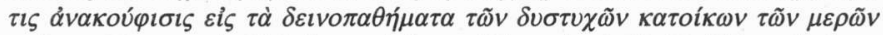

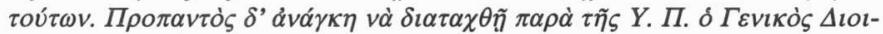

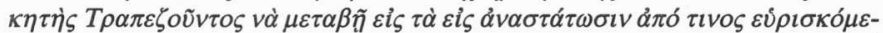

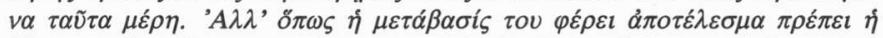

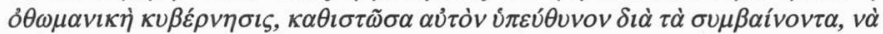

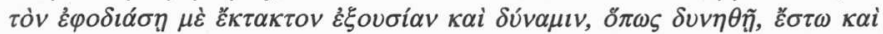

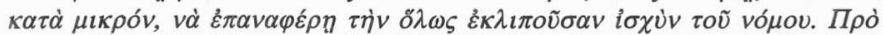

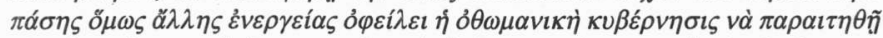

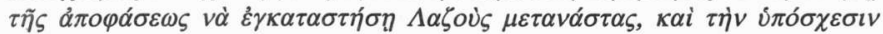

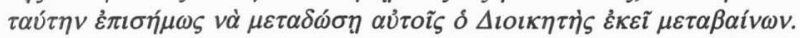

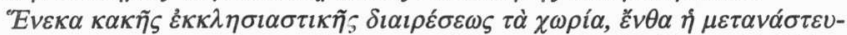

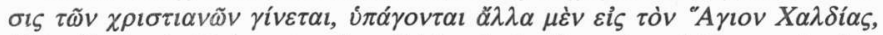

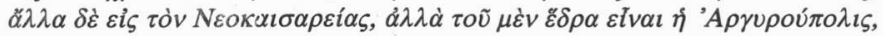

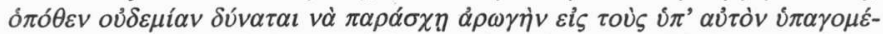

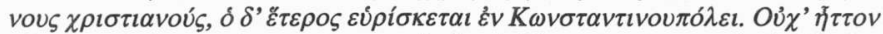

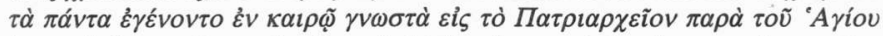

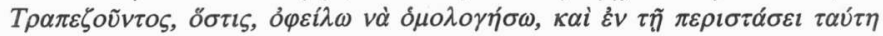

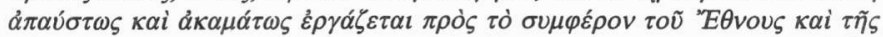

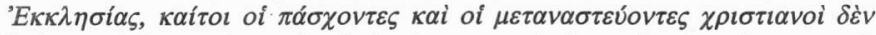

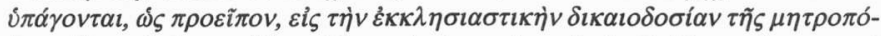

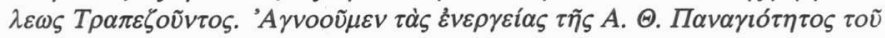

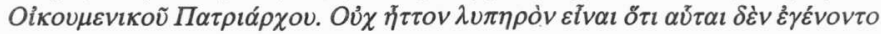




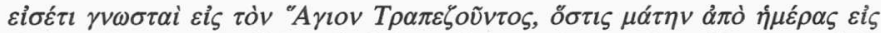

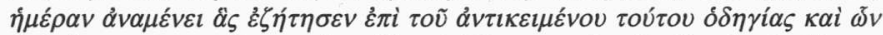

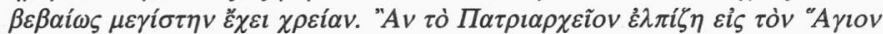

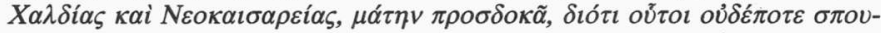

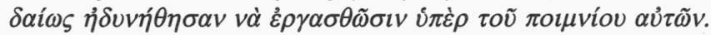

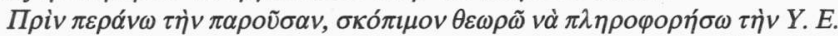

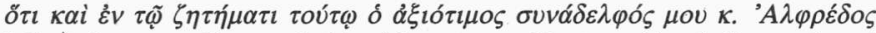

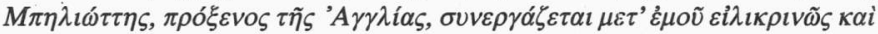

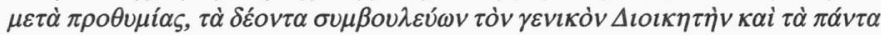

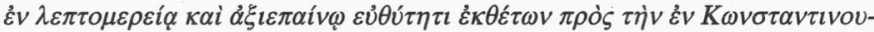

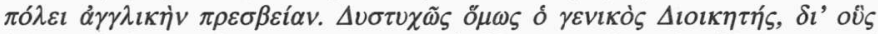

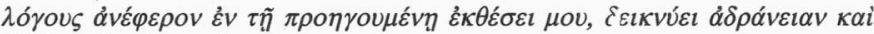

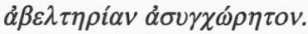

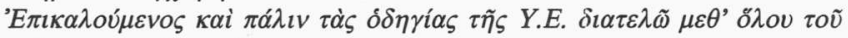

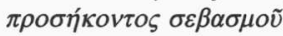

Eủ $\pi \varepsilon \imath \theta \varepsilon \dot{\sigma} \sigma \alpha \tau \sigma \varsigma$

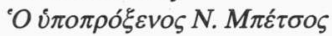

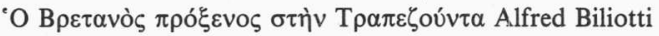

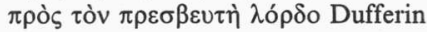

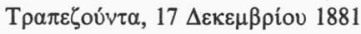

[P.R.O./F.O. 195/1381 (1881), ápt $\theta . \pi \rho \omega \tau .72]$

My Lord,

I have the honour to inform Your Lordship, with reference to my despatches no 65 of the 29th ultimo and no 68 of the 3rd instant, that the Greek Consul has not yet received the slightest instruction from his Government with regard to Greek emigrants, and that the movement of emigration is as strong as before.

The 50 Greek families, which were waiting at Ordoo, receiving no information from the Greek Consul at Trebizond, lately removed to Batoom.

It is reported from Ordoo that many emigrants, who have lately arrived from the interior, with a view to remove to Greece, are greatly sufferig from privations. Their number is not given, but they seem to be part of the 500 families from Sivas, which were expected at Samsoon. They state that should they fail to soon obtain an answer as to whether they may be sent to Greece, they will find themselves under the necessity, much against their will, to proceed to Russia.

The Greek Consular Agent at Samsoon reports that upwards of 1.000 souls 
have given him information of their intention to emigrate at the end of February next from Tach Ova, vilayet of Sivas. And a delegate from Niksar has informed the Greek Consul here that 300 families from that locality are ready to depart in March next.

In both cases the intended emigrants give notice that they are willing to go to Greece, but should they obtain no answer before the forementioned dates, as they made up their mind to leave Turkey, they will proceed to Russia.

I am informed from Batoom that the emigration in that town have not been removed to the interior, but that convenient lodging has been provided to them by the local authorities and that many have found employment in the new constructions, which are erected there, but that there are still numbers of women and children entirely depending on public charity.

The 40 Greek families which on their return from Batoom to Ordoo had been locked up in a han, in order to be sent back to Russia, have been allowed by the local authorities to remain in Turkey.

I have the honour to be with the greatest respect, My Lord,

Your Lordship's most obedient, humble servant

Alfred Biliotti

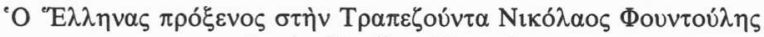

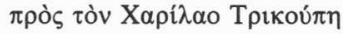

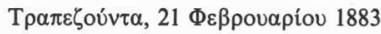

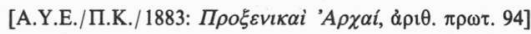

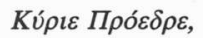

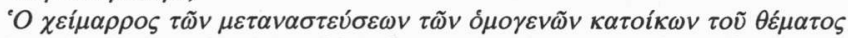

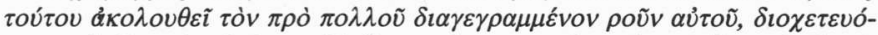

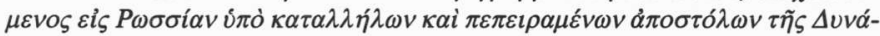

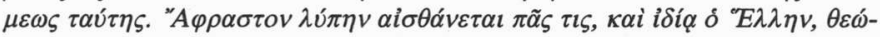

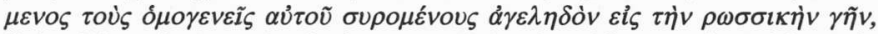

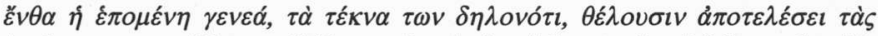

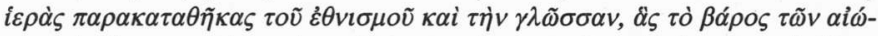

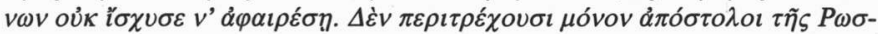

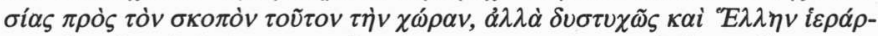

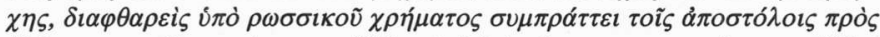

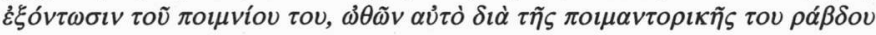

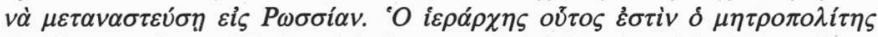

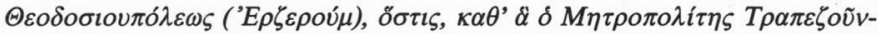

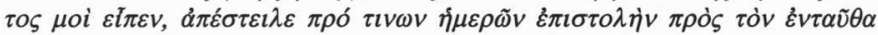

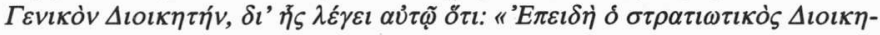




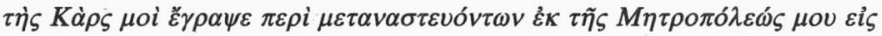

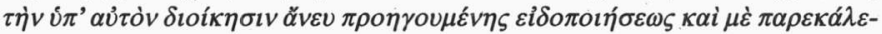

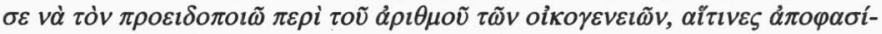

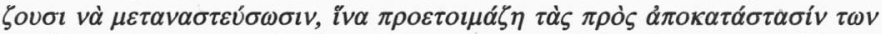

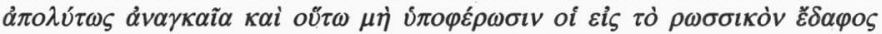

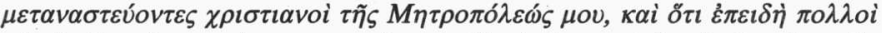

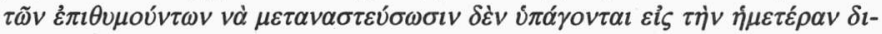

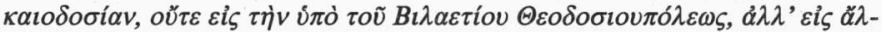

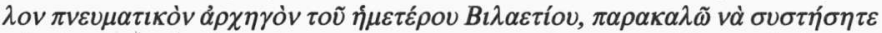

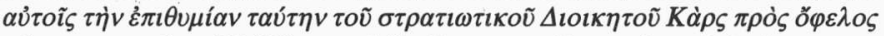

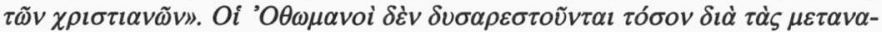

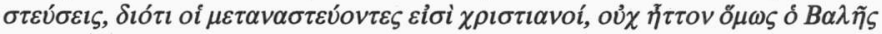

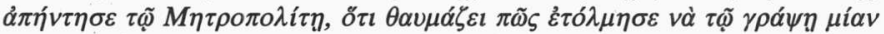

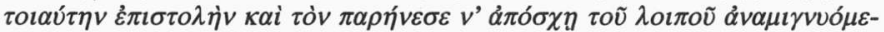

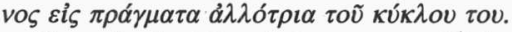

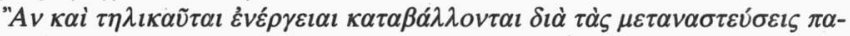

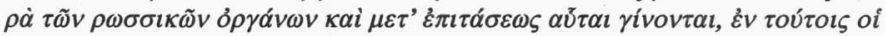

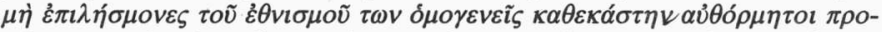

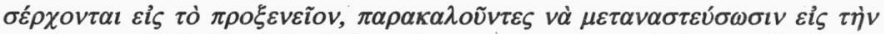

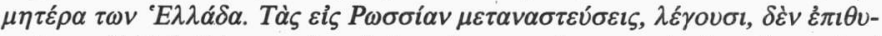

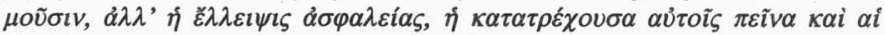

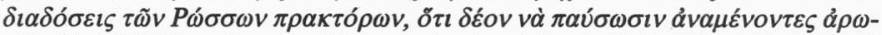

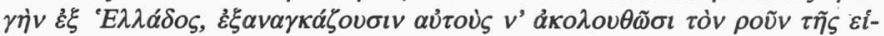

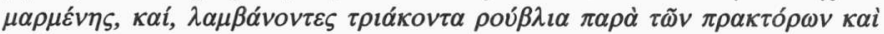

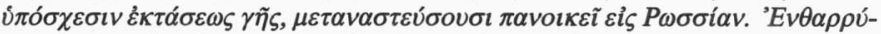

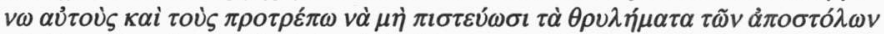

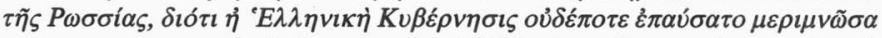

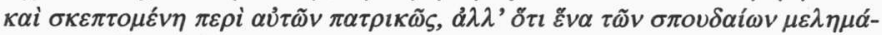

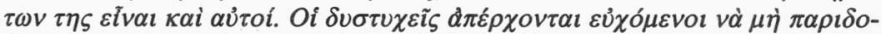
$\theta \tilde{\omega} \sigma \iota v$ oi $\pi \hat{\theta} \theta$ o $\tau \omega v$.

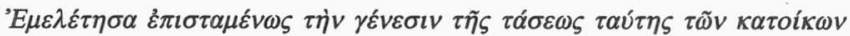

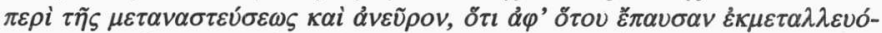

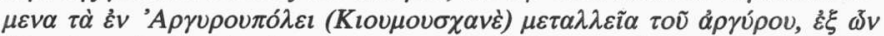

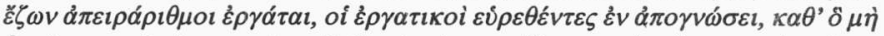

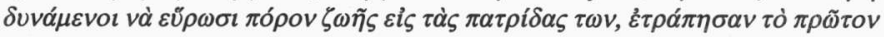

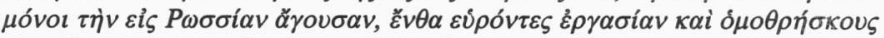

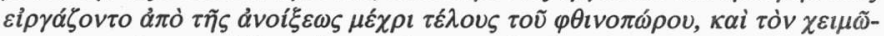

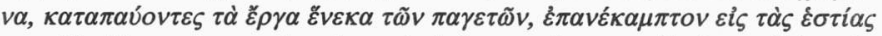

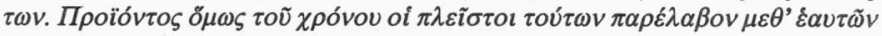

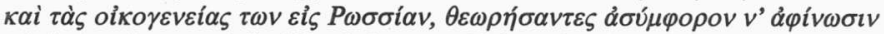

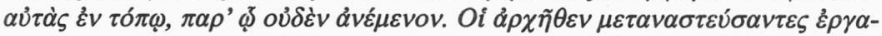

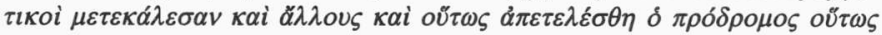

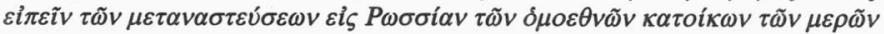




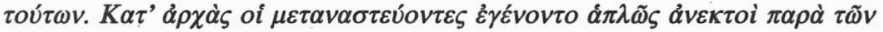

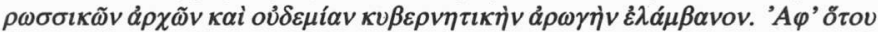

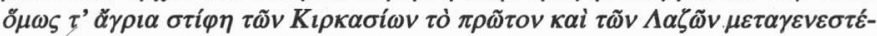

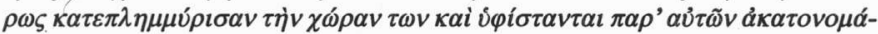

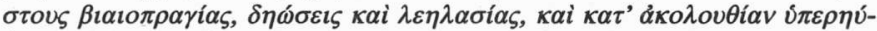

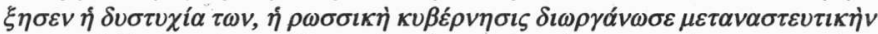

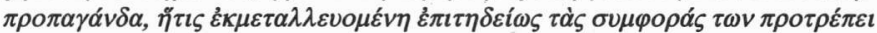

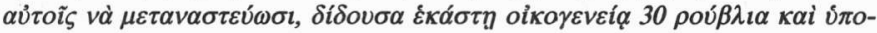

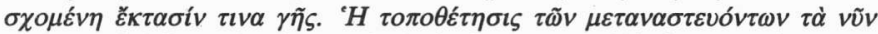

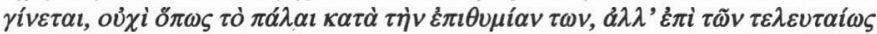

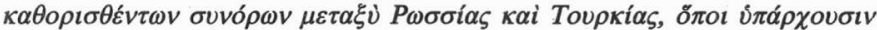

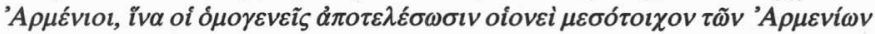

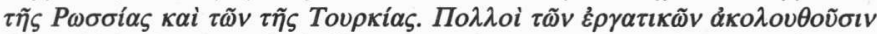

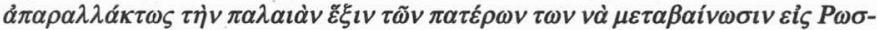

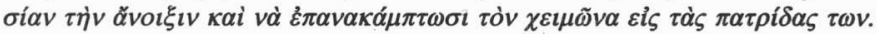

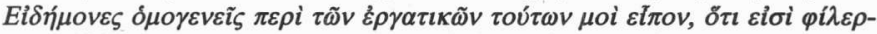

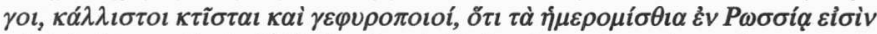

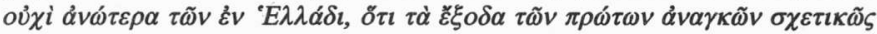

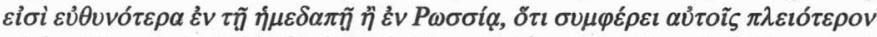

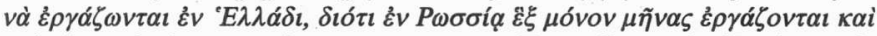

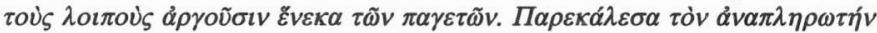

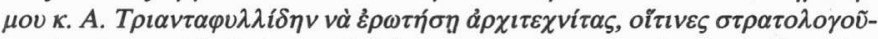

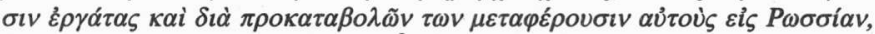

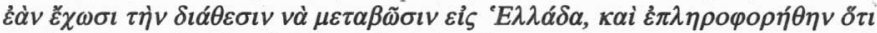

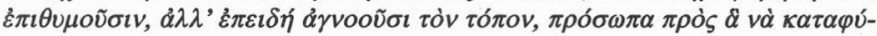

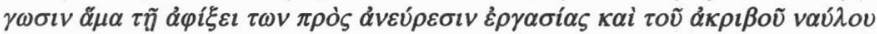

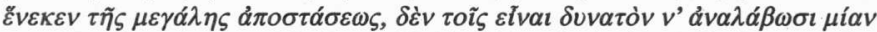

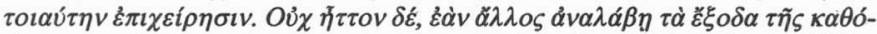

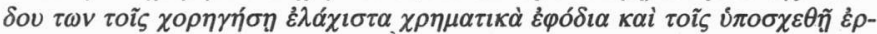
$\gamma \alpha \sigma i \alpha v, \pi \circ \lambda \lambda \circ i \quad \pi \rho \circ \theta \dot{\mu} \mu \omega \varsigma \theta \dot{\alpha} \mu \varepsilon \tau \alpha \beta \tilde{\omega} \sigma t v$.

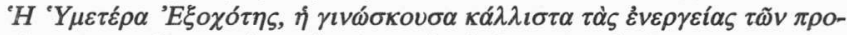

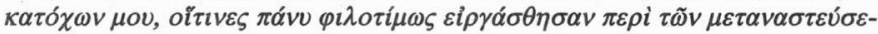

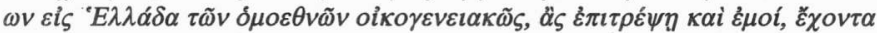

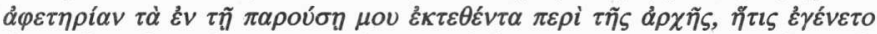

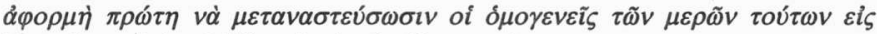

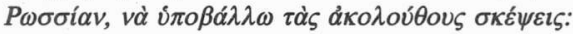

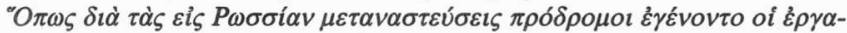

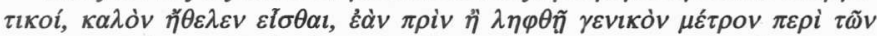

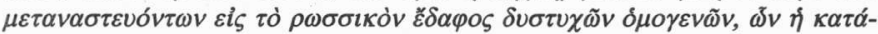

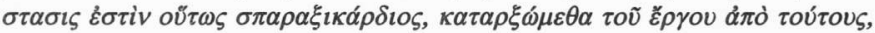

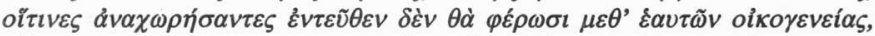

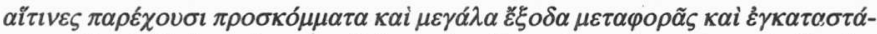

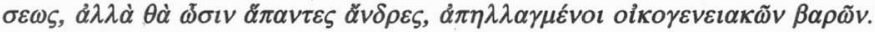




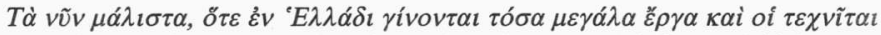

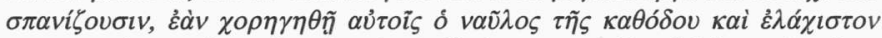

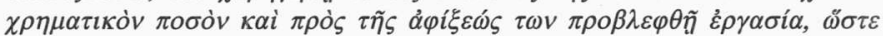

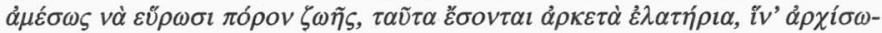

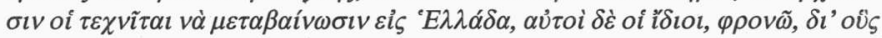

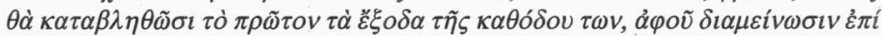

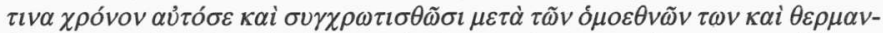

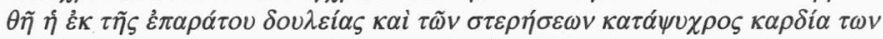

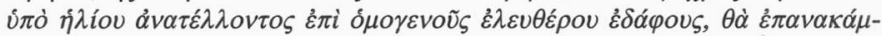

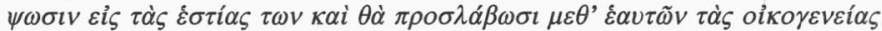

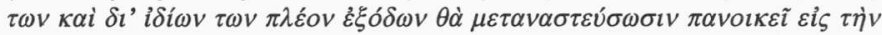

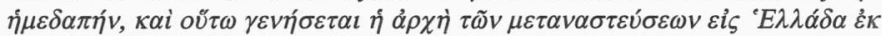

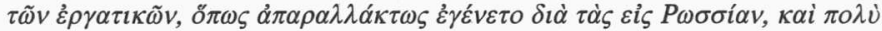

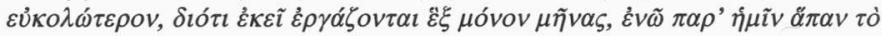

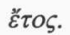

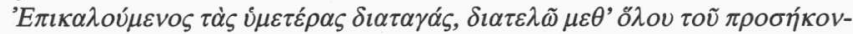
$\tau \circ \varsigma \sigma \varepsilon \beta \alpha \sigma \mu o \tilde{v}$.

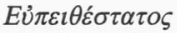

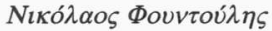




\section{ПАРАРТНMA В'}

\section{MINAKE $\Sigma$}

1

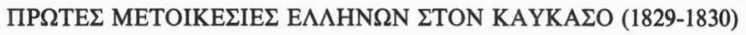

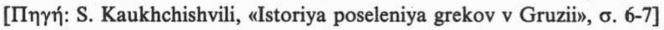

\begin{tabular}{|c|c|c|c|c|c|}
\hline$\alpha / \alpha$ & $\pi \rho \circ \varepsilon ́ \lambda \varepsilon v \sigma \eta$ & 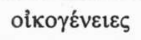 & ă $\tau о \mu \alpha$ & 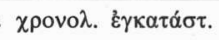 & 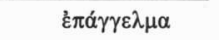 \\
\hline \multirow[t]{5}{*}{1.} & Gümushane & 85 & 517 & 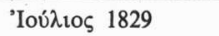 & $\mu \varepsilon \tau \alpha \lambda \lambda \omega \rho v ́ \chi 0 \imath$ \\
\hline & $"$ & & 12 & 7.5 .1830 & $n$ \\
\hline & $»$ & 46 & 121 & 7.5 .1830 & $»$ \\
\hline & $"$ & 2 & 10 & Málos 1830 & $\|$ \\
\hline & $"$ & 3 & 6 & 'Ioúvios 1830 & 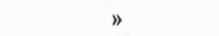 \\
\hline \multirow[t]{3}{*}{2.} & Erzurum & 26 & 96 & Málos 1830 & $\tau \varepsilon \chi v i ́ \tau \varepsilon \varsigma$ \\
\hline & $"$ & 4 & & Máıos 1830 & 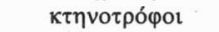 \\
\hline & $n$ & 43 & 143 & Málo̧ 1830 & $\tau \varepsilon \chi v i ́ \tau \varepsilon \zeta$ \\
\hline 3. & Kyaryak & 20 & 22 & 22 Maîo 1830 & 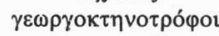 \\
\hline \multirow[t]{2}{*}{4.} & Sanamer & 11 & 89 & $"$ & $\gamma \varepsilon \omega \rho \gamma o i$ \\
\hline & 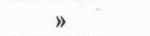 & 9 & 63 & $"$ & ") \\
\hline \multirow[t]{2}{*}{5.} & Akhalykh & 3 & 42 & $"$ & $»$ \\
\hline & " & 4 & 20 & $"$ & $"$ \\
\hline 6. & Dzhinisi & 22 & 153 & $»$ & 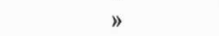 \\
\hline 7. & Khadyk к.વ̆. & 5 & 26 & $"$ & 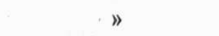 \\
\hline
\end{tabular}

2

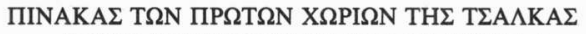

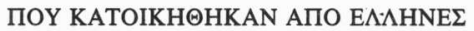

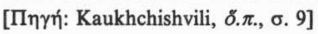

\begin{tabular}{|c|c|c|c|}
\hline \multirow[t]{2}{*}{ 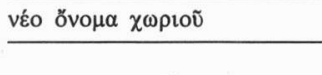 } & \multirow[t]{2}{*}{ 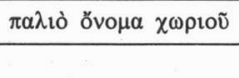 } & \multicolumn{2}{|c|}{ 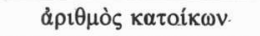 } \\
\hline & & 19.12 .1830 & 30.1 .1831 \\
\hline 1. Beshtasheni & Beshkenashen & 200 & 200 \\
\hline 2. Shipiakli (Shipiak) & Edzeni Sopeli & 130 & 132 \\
\hline 3. Kyaryak & Nizhni Halsheny & 205 & 249 \\
\hline 4. Bashkoi-Kendigi & & 240 & 240 \\
\hline 5. Khadyk-Sanamer-Akhalyk & Kochti & 265 & 265 \\
\hline 6. Barmaksiz & & 401 & 401 \\
\hline 7. Dzhinisi & Karusheti & 194 & 190 \\
\hline 8. Avranli & Tezi & 173 & 180 \\
\hline 9. Guniya & Chanchkabezha & 159 & 160 \\
\hline
\end{tabular}


3

ПINAKA $\Sigma$ EГKATA $\Sigma T A \Sigma E \Omega N$ T $\Omega N$ E $\Lambda \Lambda H N \Omega N$

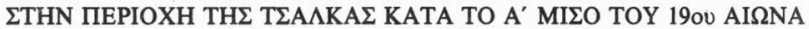

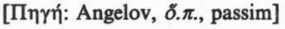

\begin{tabular}{|c|c|c|c|c|}
\hline 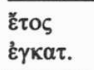 & 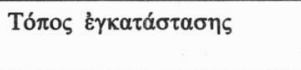 & 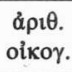 & $\pi \rho \circ \varepsilon ́ \lambda \varepsilon v \sigma \eta$ & $\pi \alpha \rho \alpha \tau \eta \rho \dot{\sigma \varepsilon} 1 \zeta$ \\
\hline 1813 & Tsintskaro & 19 & $\pi \varepsilon \rho$. П $\alpha \sigma \dot{\varepsilon} \nu$ & 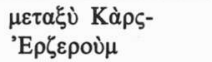 \\
\hline 1814 & $n$ & 76 & $"$ & 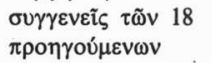 \\
\hline $1822-3$ & " & 100 & Gümushane & \\
\hline 1825 & Demir Bulakh & 15 & Erzerum & 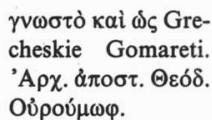 \\
\hline $1825-7$ & Keivan Bulgason & - & 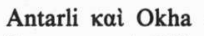 & $\pi \varepsilon \rho 10 \chi \eta े ~ ' Е \rho \zeta \varepsilon \rho \circ ৩ े \mu$ \\
\hline $1829-40$ & Ambarlo & - & 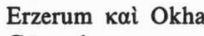 & \\
\hline 1830 & Beshtashen (Beshkenashen) & 85 & Gümushane & $\mu \varepsilon \tau \alpha \lambda \lambda \omega \rho v ́ \chi 01$ \\
\hline 1830 & $\begin{array}{l}\text { Bashkoi } \\
\text { (Berkhnii Akhalsheni) }\end{array}$ & 34 & Bashkoi & 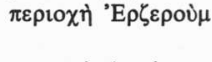 \\
\hline 1830 & Koteliya (Rekha) & - & Koteliya & 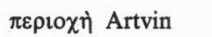 \\
\hline 1830 & Mamula Sopeli & 16 & & \\
\hline 1830 & Evreni î Avranli (Tezi) & 49 & - & 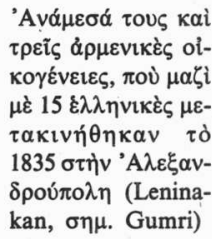 \\
\hline 1830 & Barmaksiz (Edzani) & - & Erzerum & - \\
\hline 1830 & Dzhinis & - & - & 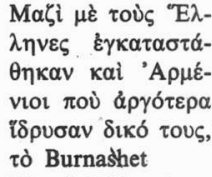 \\
\hline 1830 & $\begin{array}{l}\text { Khadyk-Sanamer-Akhalyk } \\
\text { (Kokhta î Kokhti) }\end{array}$ & & - & 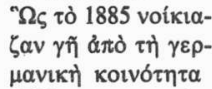 \\
\hline 1830 & $\begin{array}{l}\text { Goryak î Kyaryak } \\
\text { (Nizniy Akhalshen) }\end{array}$ & - & - & 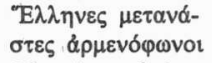 \\
\hline 1830 & $\begin{array}{l}\text { Shepyak ฑै Shipyak } \\
\text { (Edvena Sopeli) }\end{array}$ & 1 & & 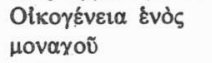 \\
\hline
\end{tabular}




\begin{tabular}{|c|c|c|c|}
\hline $\begin{array}{l}\text { हैं } \alpha \varsigma \\
\varepsilon \gamma \kappa \alpha \tau .\end{array}$ & 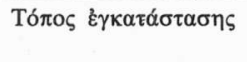 & 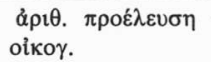 & $\pi \alpha \rho \alpha \tau \eta \rho \eta ́ \sigma \varepsilon \iota \varsigma$ \\
\hline
\end{tabular}

$\begin{aligned} 1830 & \begin{array}{l}\text { Guniya-Kala } \\ \text { (Chin Khabezha iे } \\ \text { Chanchkabezha) }\end{array} \\ 1830-40 & \text { Kumbet (Kakhadum) } \\ 1830-40 & \text { Olyank (Ayazmadar) }\end{aligned}$

4

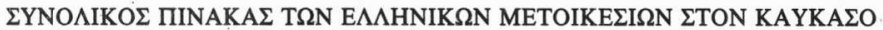
(1878-1882)

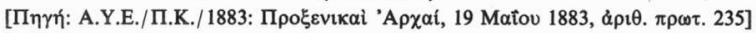

\begin{tabular}{|c|c|c|}
\hline$\Pi \rho \circ \varepsilon \hat{\lambda} \varepsilon \cup \sigma \eta$ & 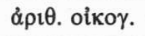 & 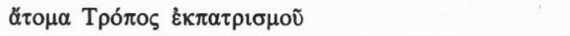 \\
\hline 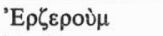 & 300 & $1.800 \Delta \mathrm{t} \dot{\alpha} \xi \eta \rho \tilde{c} \zeta$ \\
\hline 'A $\mu \alpha ́ \sigma \varepsilon \imath \alpha$ & 300 & 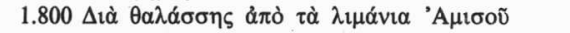 \\
\hline 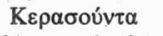 & 200 & 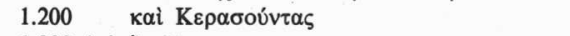 \\
\hline 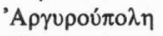 & 300 & $1.800 \Delta \mathrm{i} \dot{\alpha} \xi_{\eta} \rho \tilde{\alpha} \varsigma$ \\
\hline 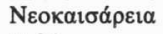 & 400 & 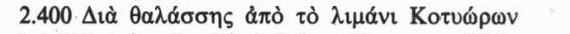 \\
\hline$\Sigma \varepsilon \beta \alpha ́ \sigma \tau \varepsilon i \alpha$ & 650 & 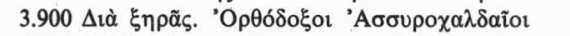 \\
\hline 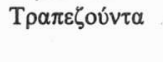 & 700 & 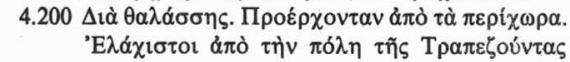 \\
\hline
\end{tabular}

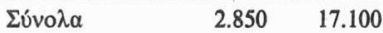

5

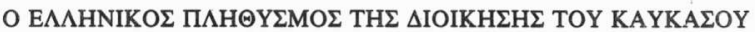

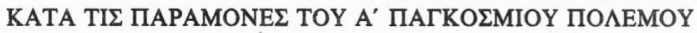

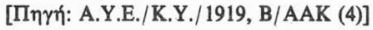

\begin{tabular}{|c|c|c|c|c|c|c|}
\hline 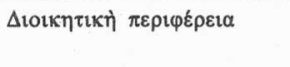 & 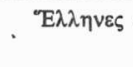 & 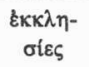 & 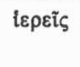 & $\sigma \chi \circ \lambda \varepsilon \tilde{\alpha} \alpha$ & 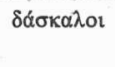 & $\begin{array}{l}\sigma 0 ́ v o \lambda o \\
\pi \lambda \eta \theta .\end{array}$ \\
\hline 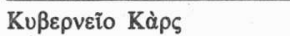 & 48.994 & 67 & 67 & 78 & 123 & 370.000 \\
\hline 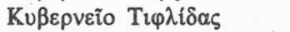 & 50.306 & 26 & 27 & 24 & 29 & 1.150 .000 \\
\hline 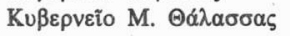 & 16.682 & 3 & 3 & 7 & 11 & 150.000 \\
\hline 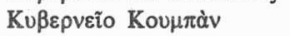 & 28.300 & 6 & 6 & 14 & 26 & 2.500 .000 \\
\hline \multicolumn{7}{|l|}{ 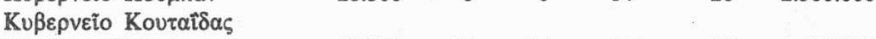 } \\
\hline 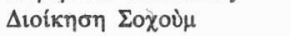 & 20.095 & 14 & 14 & 18 & 25 & 1.125 .000 \\
\hline 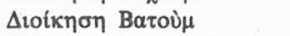 & 7.469 & 2 & 4 & 2 & 10 & 170.000 \\
\hline 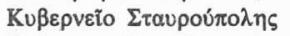 & 2.801 & 1 & 2 & 1 & 2 & 1.200 .000 \\
\hline 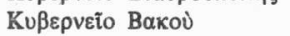 & 1.500 & - & - & - & - & 1.000 .000 \\
\hline
\end{tabular}




\begin{tabular}{|c|c|c|c|c|c|c|}
\hline 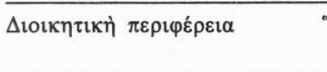 & ${ }^{\circ} \mathrm{E} \lambda \lambda \eta v \varepsilon \varsigma$ & $\begin{array}{c}\varepsilon \kappa \kappa \lambda \eta- \\
\sigma i \varepsilon \varsigma\end{array}$ & 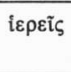 & $\sigma \chi \sigma \lambda \varepsilon i \tilde{\alpha}$ & $\delta \alpha ́ \sigma \kappa \alpha \lambda o \imath$ & 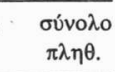 \\
\hline 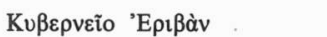 & 2.210 & 3 & 3 & 3 & 6 & 1.000 .000 \\
\hline 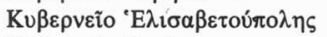 & 661 & - & - & - & - & 1.000 .000 \\
\hline 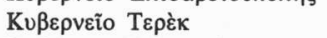 & 950 & 1 & 1 & 1 & 2 & 1.150 .000 \\
\hline 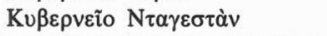 & 155 & - & - & - & - & 670.000 \\
\hline$\Sigma u ́ v o \lambda \alpha$ & 180.123 & 123 & 127 & 148 & 234 & 11.460 .000 \\
\hline
\end{tabular}

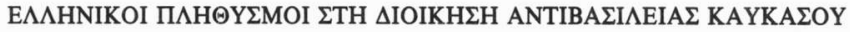

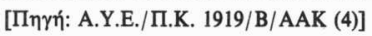

$\alpha / \alpha$ ŏvo $\mu \alpha$

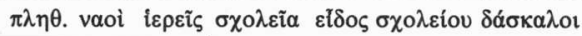

\begin{tabular}{|c|c|c|c|c|c|c|}
\hline \multicolumn{7}{|c|}{ 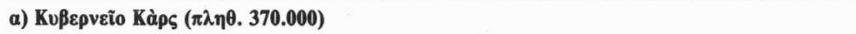 } \\
\hline 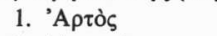 & 858 & 1 & 1 & 1 & 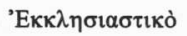 & 3 \\
\hline 2. 'Арбعvغेк & 247 & 1 & 1 & 1 & 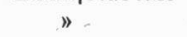 & 1 \\
\hline 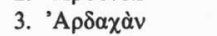 & 325 & 1 & 1 & - & - & - \\
\hline 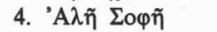 & 582 & 1 & 1 & 1 & $»$ & 2 \\
\hline 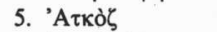 & 631 & & & & & \\
\hline 6. 'A $A 0 u \lambda \beta \alpha \grave{\rho} \tau$ & 662 & 1 & 1 & 1 & 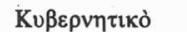 & 2 \\
\hline 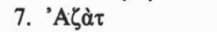 & 824 & 1 & 1 & 1 & 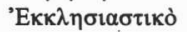 & 3 \\
\hline 8. B $\alpha \rho \gamma \kappa \varepsilon v i \varsigma$ & 525 & 1 & 1 & 1 & $"$ & 1 \\
\hline 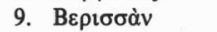 & 484 & 1 & 1 & 1 & $»$ & 1 \\
\hline 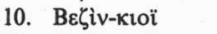 & 850 & 1 & 1 & 1 & $»$ & 3 \\
\hline 11. $\Gamma \iota \eta \lambda \iota \alpha \mu \pi \varepsilon \rho \tau$ & 970 & - & - & 1 & 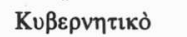 & 3 \\
\hline 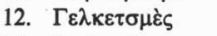 & 860 & 1 & 1 & 1 & $"$ & 2 \\
\hline 13. Гevíkıö̈ & 830 & 1 & 1 & 1 & 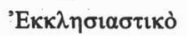 & 3 \\
\hline 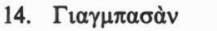 & 578 & 1 & 1 & 1 & $»$ & 2 \\
\hline 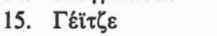 & 650 & - & - & 1 & $"$ & 1 \\
\hline 16. $\Gamma\llcorner\alpha \lambda \alpha o \nu \zeta-\tau \sigma \grave{\alpha} \mu$ & 550 & 1 & 1 & 1 & $"$ & 3 \\
\hline 17. 'Е & 570 & 1 & 1 & 1 & $»$ & 1 \\
\hline 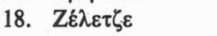 & 852 & - & - & 1 & $"$ & 1 \\
\hline 19. Z $Z \mu \mu \zeta \varepsilon \lambda \varepsilon \dot{~}$ & 352 & 1 & 1 & 1 & 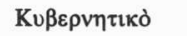 & 1 \\
\hline 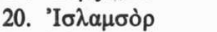 & 660 & 1 & 1 & 1 & " & 1 \\
\hline 21. 'I $\beta \grave{\alpha} v-\mu \pi 0 \lambda$ & 792 & 1 & 1 & 1 & 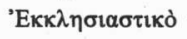 & 1 \\
\hline 22. K $\alpha \gamma \gamma \sigma \mu \alpha v$ & 85 & - & 一 & 一 & - & 一 \\
\hline 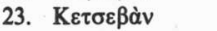 & 335 & 1 & 1 & - & - & - \\
\hline 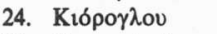 & 500 & - & - & 1 & 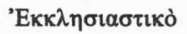 & 2 \\
\hline 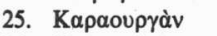 & 650 & 1 & 1 & 1 & " & 3 \\
\hline 26. Kapaкоט̀ $\rho \tau$ & 630 & 1 & 1 & 1 & $"$ & 2 \\
\hline 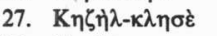 & 611 & 1 & 1 & 1 & $»$ & 1 \\
\hline 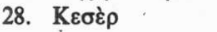 & 522 & 1 & 1 & 1 & $»$ & 1 \\
\hline
\end{tabular}




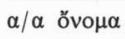

29. Kò $\gamma \kappa$

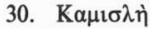

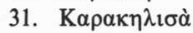

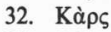

33. $\Lambda \alpha ́ \lambda \circ \gamma \lambda \eta$

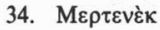

35. $\mathrm{M \varepsilon} \zeta \varepsilon \rho \varepsilon \dot{\tau}$

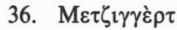

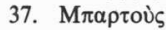

38. $\mathrm{M} \pi \varepsilon \mu \pi \varepsilon \rho \dot{\kappa} \kappa$

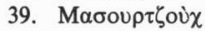

40. М $\pi \alpha \gamma \delta \dot{\alpha} \tau$

41. $М \alpha \gamma \alpha \rho \alpha \tau \zeta \grave{\kappa} \kappa$

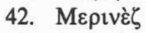

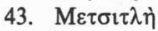

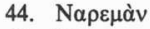

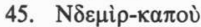

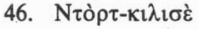

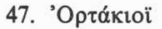

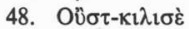

49. 'O $\mathrm{\lambda ov} \gamma \lambda \hat{\eta}$

50. "O $\mathrm{O} \tau \mathrm{V}$

51. Побغेк

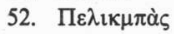

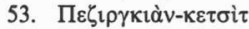

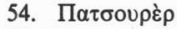

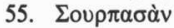

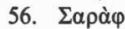

57. $\Sigma \alpha \lambda$ ov่

58. $\Sigma \alpha \lambda$ เoù

59. $\Sigma ц \mu \alpha \tau \dot{\nu} \nu$

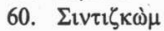

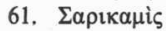

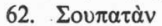

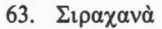

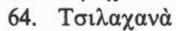

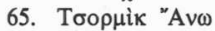

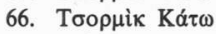

67. Тıßік

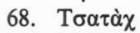

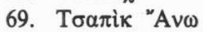

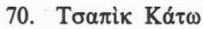

71. Т $\alpha \chi \tau \grave{\alpha}-\kappa \eta \rho \grave{v}$

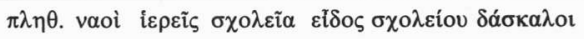

\begin{tabular}{|c|c|c|c|c|}
\hline 535 & 1 & 1 & 1 & 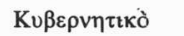 \\
\hline 650 & 1 & 1 & 1 & " \\
\hline 986 & 1 & 1 & 1 & 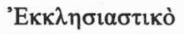 \\
\hline 1.589 & 2 & 2 & 1 & 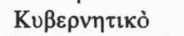 \\
\hline 757 & 1 & 1 & 1 & 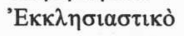 \\
\hline 305 & 1 & 1 & 1 & 》 \\
\hline 583 & 1 & 1 & 1 & " \\
\hline 794 & 1 & 1 & 1 & " \\
\hline 730 & - & - & 1 & $»$ \\
\hline 800 & 1 & 1 & 1 & 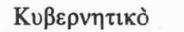 \\
\hline 485 & 1 & 1 & 1 & 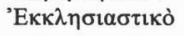 \\
\hline 326 & 1 & 1 & 1 & $»$ \\
\hline 1.600 & 1 & 1 & 1 & $»$ \\
\hline 600 & - & - & 1 & $"$ \\
\hline 795 & 1 & 1 & 1 & 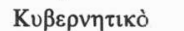 \\
\hline 679 & - & - & 1 & 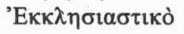 \\
\hline 312 & 1 & 1 & 1 & $»$ \\
\hline 320 & 1 & 1 & 1 & 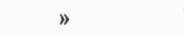 \\
\hline 588 & 1 & 1 & 1 & $"$ \\
\hline 263 & 1 & 1 & 1 & $"$ \\
\hline 708 & 1 & 1 & 1 & 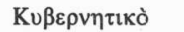 \\
\hline 400 & - & - & 1 & 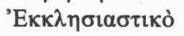 \\
\hline 344 & - & - & 1 & $"$ \\
\hline 670 & 1 & 1 & 1 & $"$ \\
\hline 623 & - & - & 1 & $"$ \\
\hline 480 & - & - & 1 & $"$ \\
\hline 360 & 1 & 1 & 1 & 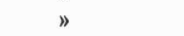 \\
\hline 511 & 1 & 1 & 1 & $»$ \\
\hline 543 & 1 & 1 & 1 & $"$ \\
\hline 225 & 1 & 1 & 1 & $»$ \\
\hline 478 & 1 & 1 & 1 & $"$ \\
\hline 878 & - & - & 1 & $"$ \\
\hline 73 & - & - & - & - \\
\hline 703 & 1 & 1 & 1 & 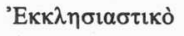 \\
\hline 300 & 1 & 1 & 1 & $»$ \\
\hline 250 & - & - & - & - \\
\hline 390 & - & - & 1 & 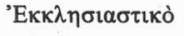 \\
\hline 417 & - & - & 1 & $»$ \\
\hline 698 & 1 & 1 & 1 & 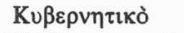 \\
\hline 500 & 1 & 1 & 1 & 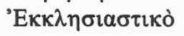 \\
\hline 512 & 1 & 1 & 1 & " \\
\hline 515 & 1 & 1 & 1 & $" 1$. \\
\hline 543 & 1 & 1 & 1 & 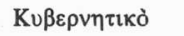 \\
\hline
\end{tabular}


$\alpha / \alpha$ ŏvou $\alpha$

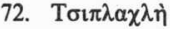

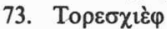

74. Тоuркабغे

75. Toü̈roùv

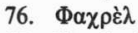

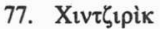

78. X Х

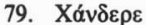

80. X Х $\alpha \grave{\alpha} \chi$

81. Х $\alpha \rho \alpha \mu \tilde{\eta} ~ \beta \alpha \rho \tau \grave{\nu}$

82. Xáбкіої

83. $X \alpha \lambda \dot{\eta} \varphi-\delta \gamma \lambda \lambda \circ v$

84. $X \alpha \tau \zeta \tilde{\eta}-\beta \varepsilon \lambda \tilde{\eta}$

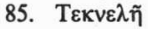

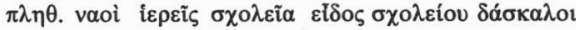

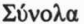

$\begin{array}{lll}355 & 1 & 1 \\ 685 & 1 & 1 \\ 650 & 1 & 1 \\ 500 & 1 & 1 \\ 470 & 1 & 1 \\ 285 & 1 & 1 \\ 231 & & \end{array}$

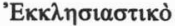

1

$600 \quad 1$

$599 \quad 1$

$\begin{array}{lll}1.197 & 1 & 1\end{array}$

$482 \quad 1 \quad 1$

207

650

280

1

1

$$
n
$$

2

"

"

"

\begin{tabular}{|c|c|c|c|c|}
\hline$\Sigma u ́ v o \lambda \alpha$ & $\begin{array}{ll}48.994 \quad 67\end{array}$ & 67 & 78 & 123 \\
\hline
\end{tabular}

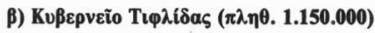

\begin{tabular}{|c|c|c|c|c|c|c|}
\hline 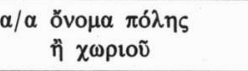 & $\pi \lambda \eta \theta$. & vooì & 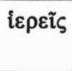 & $\sigma \chi \alpha \lambda \varepsilon \tilde{\alpha} \alpha$ & $\mu \alpha \theta \eta \tau \grave{\varepsilon} \zeta$ & $\delta \alpha ́ \sigma \kappa \alpha \lambda \circ$ \\
\hline 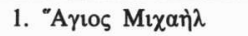 & 122 & 1 & 1 & 1 & & 1 \\
\hline 2. 'A $\mathrm{x} \alpha \lambda \tau \sigma \eta \dot{\mathrm{k}}$ & 350 & 1 & 1 & 1 & & 1 \\
\hline 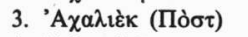 & 600 & - & - & - & & - \\
\hline 4. 'A $A \rho \alpha v \lambda \tilde{\eta}$. & 828 & 1 & 1 & 1 & $65 \mu \alpha \theta \eta \tau \dot{\varepsilon} \varsigma$ & 1 \\
\hline 5. "А $\chi \tau \varepsilon \lambda \varepsilon$ & 2.500 & & & & & \\
\hline 6. 'A & 10 & & & & & \\
\hline 7. 'А $А \alpha \beta \varepsilon ́ \rho \tau \sigma \kappa о \varepsilon$ & 3.290 & & & & & \\
\hline 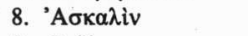 & 2.205 & & & & & \\
\hline 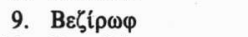 & 58 & & & & & \\
\hline 10. Варєßа́vбкоє & 45 & & & & & \\
\hline 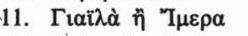 & 704 & 1 & 1 & & & \\
\hline 12. $\Gamma \varepsilon \delta \tilde{\eta}-\kappa \lambda \eta \sigma \varepsilon$ & . 850 & 1 & 1 & 1 & $5 \zeta \mu \alpha \theta \eta \tau \dot{\varepsilon} \varsigma$ & 1 \\
\hline 13. Гouvท̃-к $\alpha \lambda \varepsilon$ & 600 & 1 & 1 & 1 & $45 \mu \alpha \theta \eta \tau \dot{c} \zeta$ & 1 \\
\hline 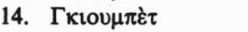 & 985 & & & & & \\
\hline 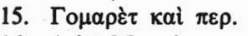 & 1.235 & & & & & \\
\hline 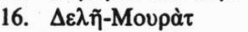 & 570 & & & & & \\
\hline 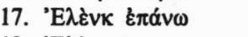 & 620 & 1 & 1 & & & \\
\hline 18. 'E 'E & 400 & 1 & 1 & 1 & $40 \mu \alpha \theta \eta \tau \dot{\xi} \varsigma$ & 1 \\
\hline 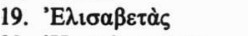 & 2 & & & & & \\
\hline 20. 'Нрака̀ $\mu \varepsilon \sigma \alpha \tilde{\alpha} \alpha$ & 260 & & & & & \\
\hline 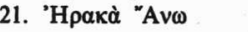 & 515 & & & & & \\
\hline
\end{tabular}




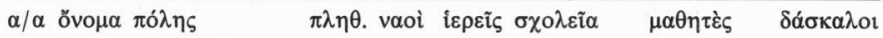
ทे $\chi \omega \rho$ เо๊̃

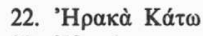

23. 'I $\beta \alpha v \omega ́ \varphi \sigma \kappa \alpha$

24. Карако̀ $\mu$

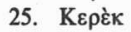

26. Koүòs

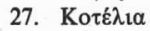

28. $\mathrm{K} \lambda \eta ́ v \delta \sigma \kappa о \varepsilon$

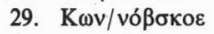

30. $\Lambda \varepsilon \imath \beta a ́ \delta ı ~$

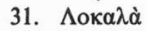

32. М $\pi \varepsilon \sigma \tau \alpha ́ \sigma\rceil$

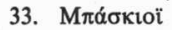

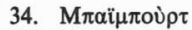

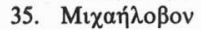

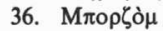

37. $\mathrm{M} \pi \alpha \rho \mu \alpha \xi i \varsigma$

38. Мí $\mu \alpha \tau$

39. $М \alpha \gamma \kappa \lambda$ กิ

40. $\mathrm{M} \tau \zeta \chi \dot{\varepsilon} \tau$

41. NÉov

42. 'О

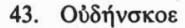

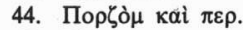

45. $\mathrm{P} \varepsilon \chi \alpha$

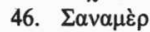

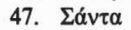

48. $\Sigma ı \pi \dot{\kappa} \kappa$

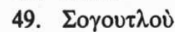

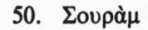

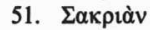

52. $\Sigma \alpha \rho \tau \alpha \tau \zeta \grave{\alpha} \lambda$

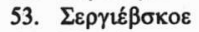

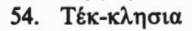

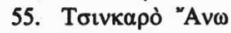

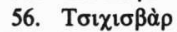

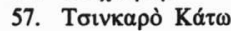

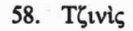

59. Tapod̀v

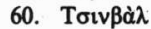

61. Тбаßки́бкоє

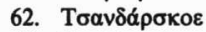

63. Tupdis

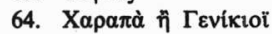

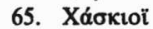

299

409

589

750

1.017

900

150

32

900

150

1.585

1.100

389

147

950

1.750

100

5

5

420

1.100

15

950

528

850

626

594

200

40

2

15

15

609

690

1.080

1.500

850

320

15

6

2.120

1.500

625

390

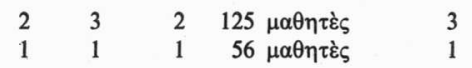

.

$\begin{array}{lll}1 & 1 & 1\end{array}$

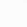

.

.

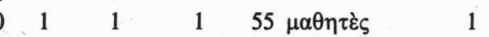

$\begin{array}{lllll}1 & 1 & 1 & 40 \mu \alpha \theta \eta \tau \grave{\varepsilon} \varsigma & 1\end{array}$

$145 \mu \alpha \theta \eta \tau \dot{\varepsilon े \zeta ~} \quad 1$

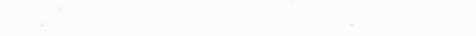

.

$\begin{array}{lllll}1 & 1 & & \\ 1 & 1 & 1 & 60 \mu \theta \eta \tau \grave{\varepsilon} \varsigma\end{array}$

$\begin{array}{lllll}1 & 1 & 1 & 1\end{array}$

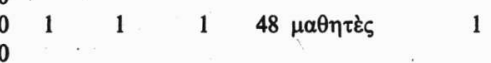




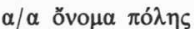

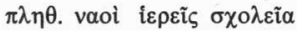

$\mu \alpha \theta \eta \tau \dot{\varepsilon} \zeta$

$\delta \alpha ́ \sigma \kappa \alpha \lambda o \imath$ กิ $\chi \omega \rho$ เoṽ

66. Xwpíov Oỉovópov 480

67. Х $\alpha \delta$ ரेк 500

68. Х $\alpha \mu \alpha \mu \lambda \eta \tilde{n}$

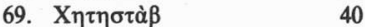

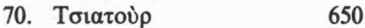

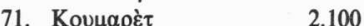

72. $\mathrm{T} \zeta \grave{\alpha} v-\mu \pi \alpha \chi \tau \sigma \dot{\varepsilon}$

73. Tбเатоن่ $\rho$

74. X X

$\begin{array}{llllll}\text { 75. इavàk } & 1.050 & 1 & 1 & 1 & 3\end{array}$

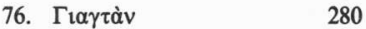

$\begin{array}{lllll}\Sigma u ́ v o \lambda \alpha & 50.306 & 26 & 27 & 24\end{array}$

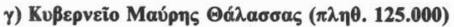

\begin{tabular}{|c|c|c|c|c|c|c|}
\hline 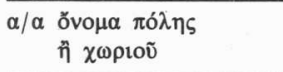 & $\pi \lambda \eta \theta$ & vaoì & 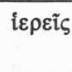 & $\chi \circ \lambda \varepsilon \tilde{\alpha} \alpha$ & 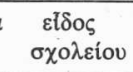 & $\delta \alpha ́ \sigma \kappa \alpha \lambda \circ$ \\
\hline 1. 'A $A \lambda \lambda \varepsilon \dot{\rho} \sigma \kappa \eta$ & 180 & 1 & 1 & 1 & $\hat{\varepsilon} \xi \alpha \tau \alpha^{\prime} \xi 10$ & 1 \\
\hline 2. 'A $А \varepsilon \rho \pi \imath \varepsilon \beta \beta \sigma \kappa о \varepsilon$ & 750 & & & & & \\
\hline 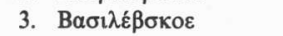 & 600 & & & & & \\
\hline 4. К К $\alpha \pi \alpha \rho \delta \dot{v} v \sigma \kappa о \varepsilon$ & 650 & & & & & \\
\hline 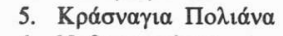 & 600 & 1 & 1 & 1 & & 1 \\
\hline 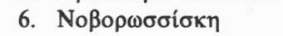 & 1.400 & & & 1 & 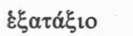 & 3 \\
\hline 7. Прабко́ßбкоє & 750 & & & & & \\
\hline 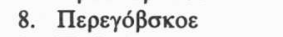 & 150 & & & & & \\
\hline 9. Пба́ंбкоє & 200 & & & & & \\
\hline 10. $\Sigma o ́ \tau \zeta \eta$ & 150 & 1 & 1 & 1 & & 1 \\
\hline 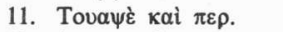 & 2.300 & & & & & \\
\hline 12. $\delta เ \varepsilon \sigma \pi \alpha \rho \mu \varepsilon ́$ voเ & 4.500 & & & & & \\
\hline 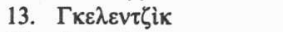 & 532 & & & 1 & & 2 \\
\hline 14. $\Lambda \alpha \zeta \alpha \rho \delta ́ \varphi \sigma \kappa \eta$ & 1.050 & & & 1 & 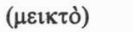 & 2 \\
\hline 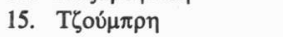 & 350 & & & 1 & & 1 \\
\hline 16. Вiбóko & 350 & & & & & \\
\hline 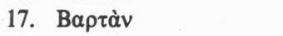 & 560 & & & & & \\
\hline 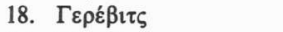 & 700 & & & & & \\
\hline 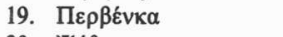 & 210 & & & & & \\
\hline 20. $\Psi \alpha \theta \alpha$ & 700 & & & & & \\
\hline$\Sigma$ Évoג $\alpha$ & 16.682 & 3 & 3 & 7 & & 11 \\
\hline
\end{tabular}




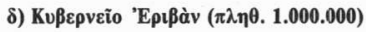

\begin{tabular}{|c|c|c|c|c|c|c|}
\hline 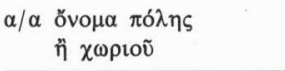 & $\pi \lambda \eta \theta$ & $\alpha o i ̀$ & 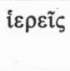 & $\sigma \chi \circ \lambda \varepsilon \tilde{\alpha} \alpha$ & 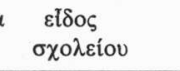 & $\delta \alpha ́ \sigma \kappa \alpha \lambda o 1$ \\
\hline 1. 'A $A \varepsilon \xi \alpha v \delta \rho \circ \pi \grave{\partial} \lambda$ & 270 & 1 & 1 & 1 & 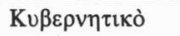 & 2 \\
\hline 2. 'A $A \varepsilon \xi \alpha v \delta \rho o \pi \mathrm{ò \lambda} \pi \varepsilon \rho$. & 850 & & & & & \\
\hline 3. 'E & 35 & & & & & \\
\hline 4. ' $E \tau \zeta \mu 1 \alpha \tau \zeta i v$ & 20 & & & & & \\
\hline 5. N $\alpha \chi \eta \tau \zeta \varepsilon \beta \dot{\alpha} \nu$ & 45 & & & & & \\
\hline 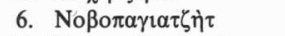 & 215 & & & & & \\
\hline 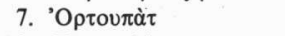 & 5 & & & & & \\
\hline 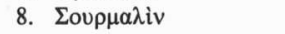 & 5 & & & & & \\
\hline 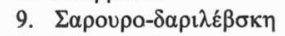 & 5 & & & & & \\
\hline 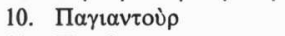 & 480 & 1 & 1 & 1 & ${ }^{e} \mathrm{I} \varepsilon \rho \alpha \tau . \mathrm{K} \cup \beta \varepsilon \rho \nu$. & 3 \\
\hline 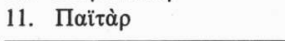 & 280 & 1 & 1 & 1 & 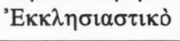 & 1 \\
\hline 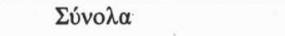 & 2.210 & 3 & 3 & 3 & & 6 \\
\hline
\end{tabular}

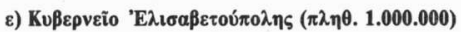

\begin{tabular}{|c|c|}
\hline 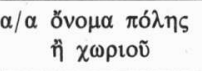 & 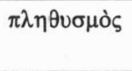 \\
\hline 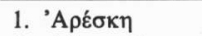 & 24 \\
\hline 2. $\Delta \zeta \varepsilon \pi \rho \alpha \eta \dot{\lambda} \lambda$ & 10 \\
\hline 3. $\Delta \zeta \varepsilon \beta \alpha \nu \sigma \eta े \rho$ & 100 \\
\hline 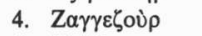 & 220 \\
\hline 5. К К $\alpha \alpha_{\alpha} \chi \sigma \kappa \eta$ & 280 \\
\hline 6. Noúxa & 12 \\
\hline 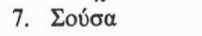 & 15 \\
\hline 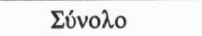 & 661 \\
\hline
\end{tabular}

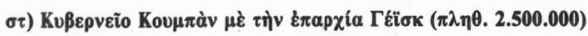

\begin{tabular}{|c|c|c|c|c|c|c|}
\hline 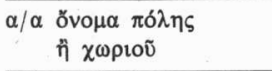 & $\pi \lambda \eta \theta$. & vaoì & 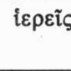 & $\varsigma \sigma \chi 0 \lambda \varepsilon \tau \alpha$ & 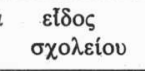 & $\delta \alpha \alpha \sigma \kappa \alpha \lambda o 1$ \\
\hline 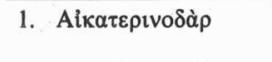 & 2.500 & 1 & 1 & 1 & $\begin{array}{l}\pi \varepsilon v \tau \alpha \tau \alpha \dot{\xi} \xi 10 \\
145 \mu \alpha \theta \eta \tau \dot{\varepsilon} \varsigma\end{array}$ & 7 \\
\hline 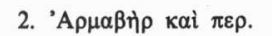 & 1.030 & & & & & \\
\hline 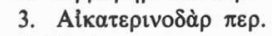 & 10.500 & & & & & \\
\hline 4. 'Avád $\pi \alpha$ & 1.540 & & & 1 & $\pi \varepsilon \nu \tau \alpha \tau \alpha \xi_{10}$ & 3 \\
\hline 5. 'А $\varphi i \pi \sigma \kappa \alpha \gamma i \alpha$ & 180 & & & 1 & & 1 \\
\hline 6. 'A $\mathrm{Aiv} \sigma \kappa \alpha \gamma 1 \alpha$ & 450 & & & 1 & & 1 \\
\hline
\end{tabular}




\begin{tabular}{|c|c|c|c|c|}
\hline 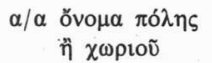 & $\pi \lambda \eta \theta$. vaoì & 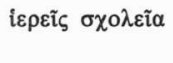 & 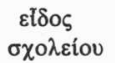 & $\delta a ́ \sigma \kappa \alpha \lambda o \imath$ \\
\hline
\end{tabular}

\begin{tabular}{|c|c|c|c|c|c|c|}
\hline 7. $B \imath \tau \iota \zeta \omega \varphi$ & 1.400 & 1 & 1 & 1 & & 1 \\
\hline 8. 'Т $\lambda \sigma \kappa \alpha \gamma 1 \alpha$ & 80 & & & 1 & & 1 \\
\hline 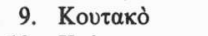 & 910 & 1 & 1 & 1 & $110 \mu \alpha \theta \eta \tau \dot{\varepsilon} \varsigma$ & 3 \\
\hline 10. К Кі́́ ${ }^{\prime} \sigma \kappa \alpha \gamma \alpha$ & 2.100 & 1 & 1 & 1 & $100 \mu \alpha \theta \eta \tau \dot{c} \varsigma$ & 2 \\
\hline 11. $M \varepsilon \rho \tau \zeta \grave{\alpha} v$ & 2.100 & 1 & 1 & 1 & 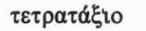 & 2 \\
\hline 12. Маїкஸे $\pi$ & 680 & & & 1 & & 1 \\
\hline 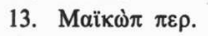 & 2.800 & & & & & \\
\hline 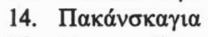 & 100 & & & 1 & & 1 \\
\hline 15. $\Sigma \tau \alpha \cup \rho о \pi o ́ \lambda \kappa \alpha$ & 450 & & & & & \\
\hline 16. $\Sigma \varepsilon \beta \hat{\varepsilon} \rho \sigma \kappa \alpha \gamma 1 \alpha$ & 250 & & & 1 & & 1 \\
\hline 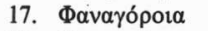 & 350 & & & & & \\
\hline 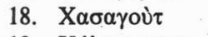 & 700 & 1 & 1 & 1 & & 1 \\
\hline 19. Хó $\lambda \mu \sigma \kappa \alpha \gamma i \alpha$ & 180 & & & 1 & & 1 \\
\hline$\Sigma u ́ v o \lambda \alpha$ & 28.300 & 6 & 6 & 14 & & 26 \\
\hline
\end{tabular}

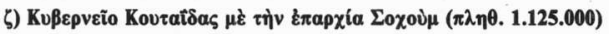

\begin{tabular}{|c|c|c|c|c|c|c|}
\hline 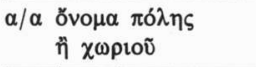 & $\pi \lambda \eta \theta$ & aoì & 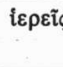 & $\sigma \chi \circ \lambda \varepsilon \tilde{\alpha} \alpha$ & 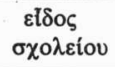 & $\delta \alpha ́ \sigma \kappa \alpha \lambda o t$ \\
\hline 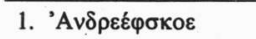 & 364 & 1 & 1 & 1 & & 1 \\
\hline 2. 'А $А \varepsilon \xi \alpha \nu \delta \rho o ́ \varphi \sigma \kappa о \varepsilon$ & 420 & 1 & 1 & 1 & & 1 \\
\hline 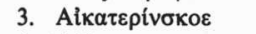 & 301 & 1 & 1 & 1 & & 1 \\
\hline 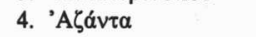 & 1.680 & 1 & 1 & 1 & & 2 \\
\hline 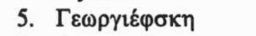 & 329 & 1 & 1 & 1 & & 1 \\
\hline 6. $\Delta \eta \mu \eta \tau \rho \imath \varepsilon \varphi \sigma \kappa \eta$ & 210 & & & & & \\
\hline 7. $\Delta \rho \alpha ́ v \delta \alpha$ & 270 & & & & & \\
\hline 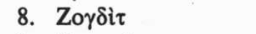 & 50 & & & & & \\
\hline 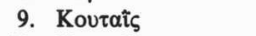 & 220 & & & 1 & & 1 \\
\hline 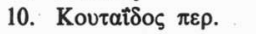 & 185 & & & & . & \\
\hline 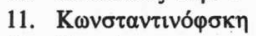 & 1.050 & 1 & 1 & 1 & & 1 \\
\hline 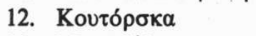 & 1.190 & 1 & 1 & 1 & & 2 \\
\hline 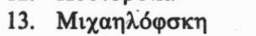 & 910 & 1 & 1 & 1 & & 1 \\
\hline 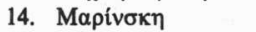 & 196 & 1 & 1 & 1 & & 1 \\
\hline 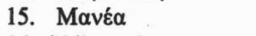 & 940 & 1 & 1 & 1 & & 1 \\
\hline 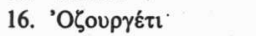 & 15 & & & & & \\
\hline 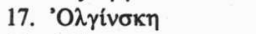 & 1.230 & 1 & 1 & 1 & & 2 \\
\hline 18. Пó $\imath$ & 350 & & & 1 & & 1 \\
\hline 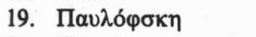 & 371 & & & & & \\
\hline 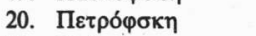 & 294 & & & & & \\
\hline 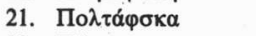 & 490 & 1 & 1 & 1 & & 1 \\
\hline 22. $\Pi \eta \rho \tau \tau \sigma \chi$ & 400 & & & & & \\
\hline
\end{tabular}




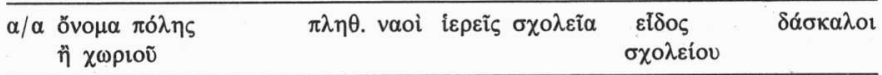

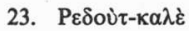
15

24. Pá $\tau \zeta \alpha$

25. $\Sigma \alpha \rho \alpha \pi \alpha ̀ v-\Pi \varepsilon \lambda o \gamma o ́ \rho 1$

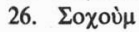

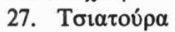

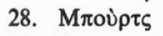

29. $\delta 1 \varepsilon \sigma \pi \alpha \rho \mu \varepsilon ́ v o เ$

30. 'О

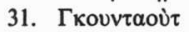

32. Гка́ $\gamma \gamma \rho \eta$

15

500

2.100

200

1.260

3.000

350

490

700

\begin{tabular}{llllll}
\hline$\Sigma$ v́voגa & 20.095 & 14 & 14 & 18 & 25 \\
\hline
\end{tabular}

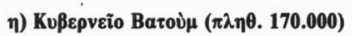

\begin{tabular}{|c|c|c|c|c|c|c|}
\hline 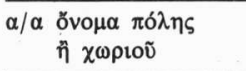 & $\pi \lambda \eta \theta$ & vooi & 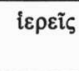 & $\sigma \chi \circ \lambda \varepsilon i \tilde{\alpha} \alpha$ & 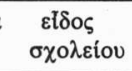 & $\delta \dot{\alpha} \sigma \kappa \alpha \lambda \mathrm{ol}$ \\
\hline 1. "А ${ }^{*} \alpha \lambda \sigma \varepsilon \nu$ & 735 & 1 & 1 & 1 & $\tau \rho ı \tau a ́ \xi ̧ ı$ & 2 \\
\hline 2. "Атбко $\beta \alpha$ & 490 & & & & & \\
\hline 3. 'A $A \tau \beta \eta \nu$ & 25 & & & & & \\
\hline 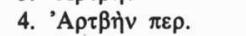 & 470 & & & & & \\
\hline 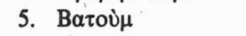 & 3.500 & 1 & 3 & 1 & $\varepsilon \pi \tau \alpha \tau \alpha^{\prime} \xi \xi o$ & 6 \\
\hline 6. $\Delta \alpha \gamma \gamma \beta \alpha$ & 602 & & & & & \\
\hline 7. $\mathrm{K} \beta \eta ́\{\eta \kappa \varepsilon$ & 420 & & & & & \\
\hline 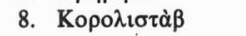 & 105 & & & & & \\
\hline 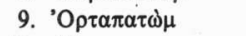 & 140 & & & & & \\
\hline 10. $\Sigma \grave{\alpha} \rho \pi$ & 30 & & & & & \\
\hline 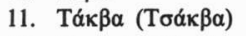 & 700 & & & & & \\
\hline 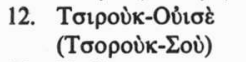 & 112 & & & & & \\
\hline 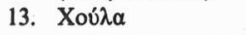 & 140 & & & & & \\
\hline$\Sigma 0 ́ v o \lambda \alpha$ & 7.469 & 2 & 4 & 2 & & 8 \\
\hline
\end{tabular}




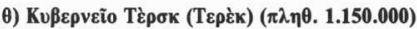

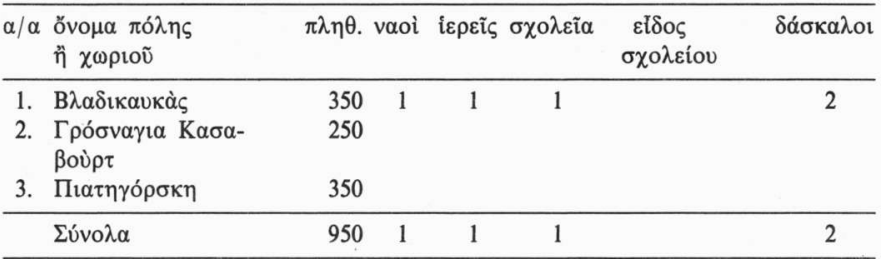

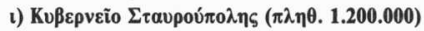

\begin{tabular}{|c|c|c|c|c|c|c|}
\hline 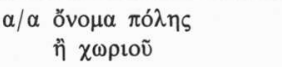 & $\pi \lambda \eta \theta$ & $\alpha o i$ & i $\varepsilon \rho \varepsilon \tilde{\mathbf{i}}$ & $\sigma \chi \circ \lambda \varepsilon \tilde{\alpha} \alpha$ & 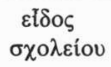 & $\delta a ́ \sigma \kappa \alpha \lambda o \imath$ \\
\hline 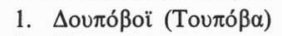 & 856 & & & & & \\
\hline 2. K $\alpha \sigma \alpha o v ́ \tau 1$ & 300 & & & & & \\
\hline 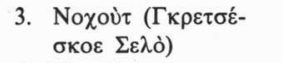 & 1.575 & 1 & 2 & 1 & & 2 \\
\hline 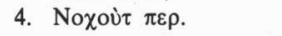 & 70 & & & & & \\
\hline$\Sigma u ́ v Q \lambda \alpha$ & 2.801 & 1 & 2 & 1 & & 2 \\
\hline
\end{tabular}

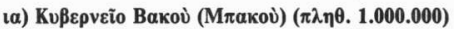

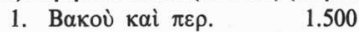

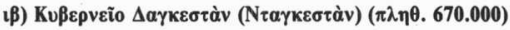

\begin{tabular}{|c|c|}
\hline 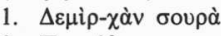 & 15 \\
\hline 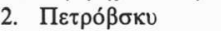 & 140 \\
\hline 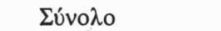 & 155 \\
\hline
\end{tabular}

7

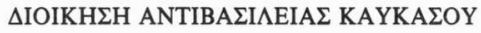

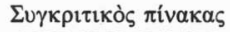

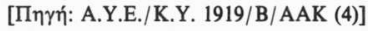

\begin{tabular}{|c|c|c|c|}
\hline 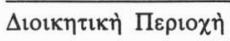 & $A^{\prime}$ & $\mathbf{B}^{\prime}$ & 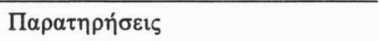 \\
\hline 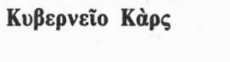 & 48.994 & 37.200 & 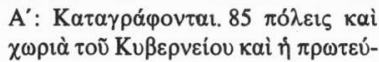 \\
\hline
\end{tabular}




\begin{tabular}{|c|c|c|c|}
\hline 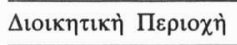 & $\mathbf{A}^{\prime}$ & $\mathbf{B}^{\prime}$ & 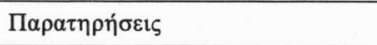 \\
\hline 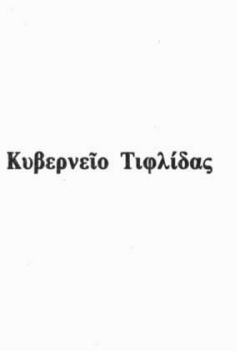 & 50.306 & 43.162 & 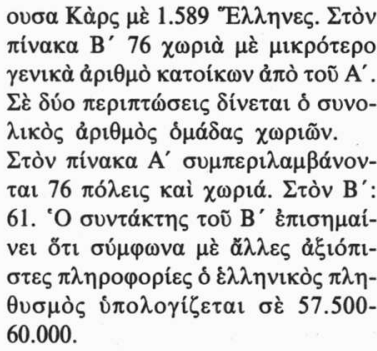 \\
\hline
\end{tabular}

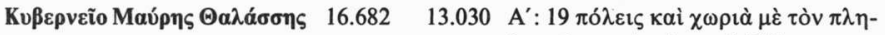

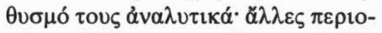

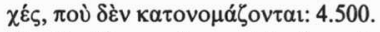

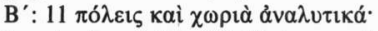

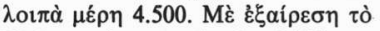

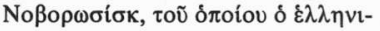

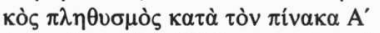

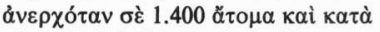

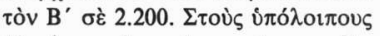

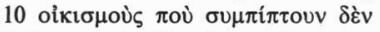

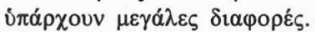

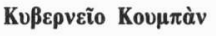

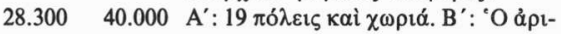

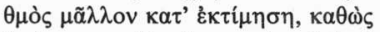

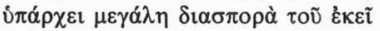

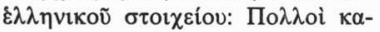

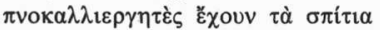

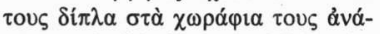

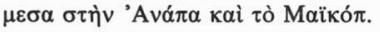

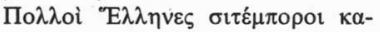

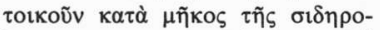

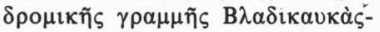
Noßоршбібк.

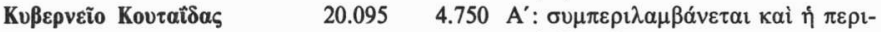

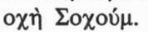

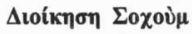

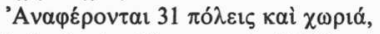

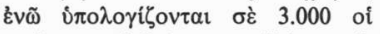

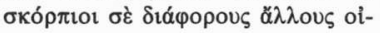

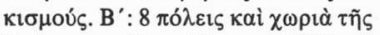

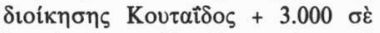

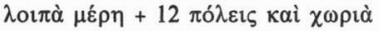

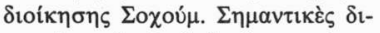

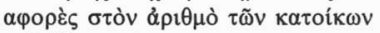

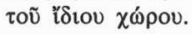




\begin{tabular}{|c|c|c|c|}
\hline 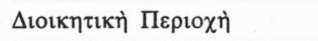 & $\mathbf{A}^{\prime}$ & $\mathbf{B}^{\prime}$ & 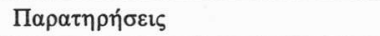 \\
\hline 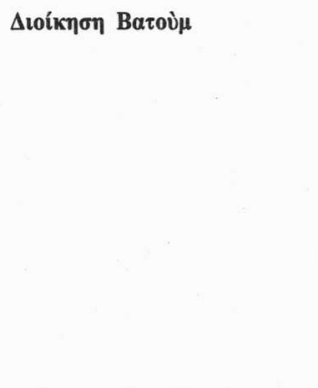 & 7.469 & 12.800 & 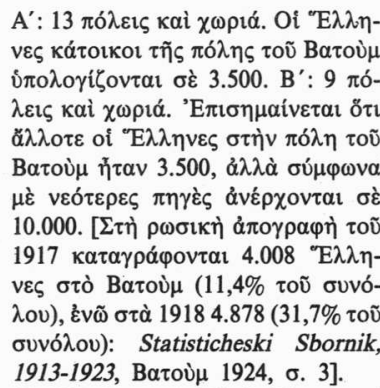 \\
\hline 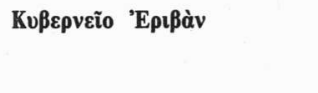 & 2.210 & 1.388 & 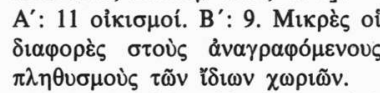 \\
\hline 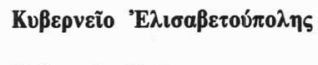 & 661 & 661 & 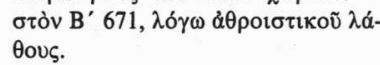 \\
\hline 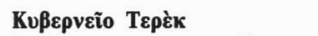 & 950 & 1.150 & \\
\hline К & 2.801 & 1.550 & \\
\hline 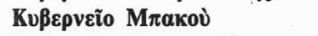 & 1.500 & 1.500 & \\
\hline 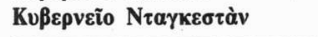 & 155 & & . \\
\hline
\end{tabular}

ПINAKA $\Sigma$ T $\Omega N$ E $\Lambda \Lambda H N I K \Omega N$ X $\Omega P I \Omega N$ TOY KYBEPNEIOY KAP $\Sigma$ ПOY YПЕ ( $\tau \dot{\varepsilon} \lambda \eta$ 1914-'I $\alpha \nu .1915)$

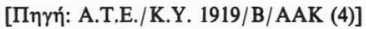

\begin{tabular}{|c|c|c|}
\hline 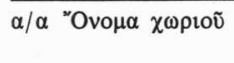 & 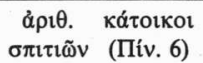 & 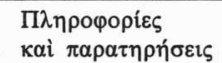 \\
\hline
\end{tabular}

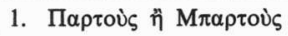

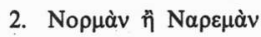

150

120

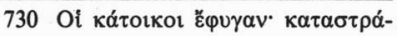

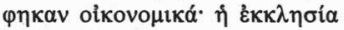
$\lambda \varepsilon \eta \lambda \alpha \tau \eta \dot{\theta} \theta \kappa \varepsilon$.

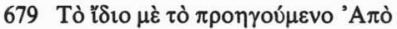

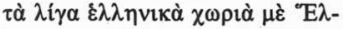

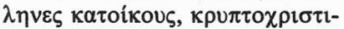

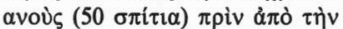

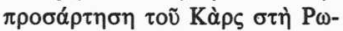

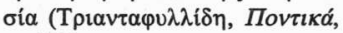
б. 127). 


\begin{tabular}{|c|c|c|c|}
\hline$\alpha / \alpha$ "Оvон $\alpha \chi \omega \rho เ о \tilde{~}$ & 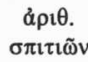 & $\begin{array}{l}\text { кátoıเко } \\
\text { (Пiv. 6) }\end{array}$ & $\begin{array}{l}\Pi \lambda \eta \rho о \varphi о \rho і \varepsilon \varsigma \\
\text { каi } \pi \alpha \rho \alpha \tau \eta \eta \dot{\sigma \varepsilon \varepsilon \varsigma}\end{array}$ \\
\hline
\end{tabular}

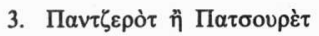 \\ 4. Поơik

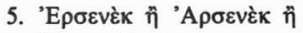 'Apбevvàk

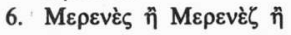 Mepıvย̀

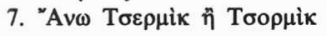

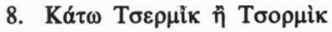

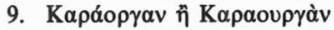

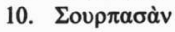 \\ 11. Гeví-Kıö \\ 12. Kı́ópinov

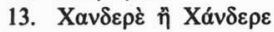

14. Xáokioï

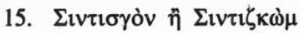

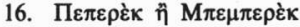

17. B $\alpha \gamma \delta \dot{\alpha} \tau$ iे $\mathbf{M} \pi \alpha \gamma \delta \dot{\alpha} \tau$

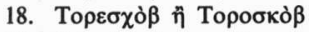

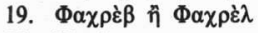

20. Xavàx

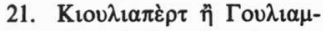
$\pi \dot{\rho} \rho \tau$ ฑิ $\Gamma \kappa 100 \lambda \varepsilon \mu \pi \varepsilon \dot{\rho} \rho \tau$

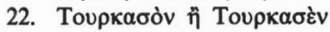

23. KòyK

24. $\Sigma \varepsilon \mu \sigma \varepsilon \lambda \varepsilon \dot{~}$

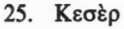

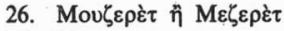

27. Мвртіvik

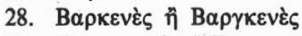

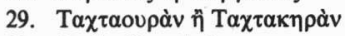

30. $\Delta \varepsilon \mu i \rho-K \alpha \pi \circ$ iे ทे

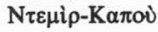

60

50

80

50

50

40

70

50

80

50

55

90

180

50

70

45

50

220

130

80

70

60

70

65

$85 \quad 525$

$110 \quad 543$

$90 \quad 312$

878

800

326

685

470

599

970

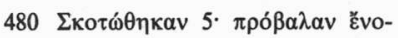

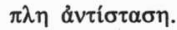

344 "A

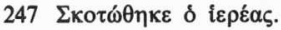

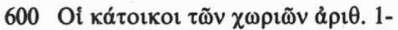

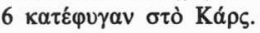

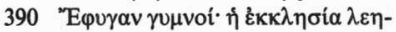
$\lambda \alpha \tau \dot{\theta} \theta \eta \kappa \varepsilon$.

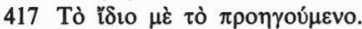

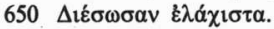

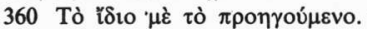

830 Tò ť́

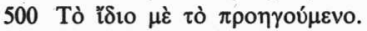

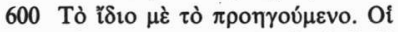

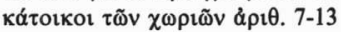

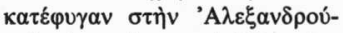

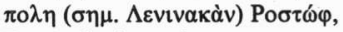

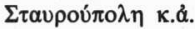

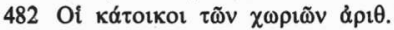
14-21 है $\varphi$

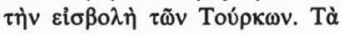

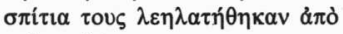

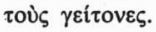

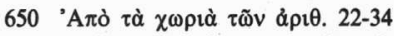

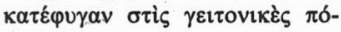
$\lambda \varepsilon\llcorner\varsigma \mu \grave{\varepsilon} \lambda \hat{i} \gamma \alpha \quad \pi \rho \alpha ́ \gamma \mu \alpha \tau \alpha$. 'H '́k-

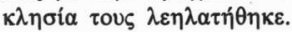




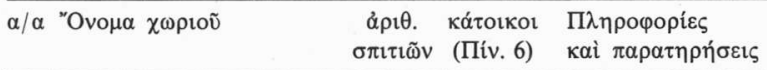

31. Oü $\varsigma-K ı \lambda เ \sigma \varepsilon े$

32. $\Sigma \alpha \lambda$ ov̀

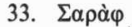

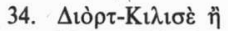
$\mathrm{N} \tau$ ò $\rho \tau \mathrm{K} \iota \lambda \iota \sigma \grave{\varepsilon}$

35. Вepiбàv

36. $\Lambda \alpha \dot{\lambda} \circ \gamma \lambda \eta$

37. $\Sigma \alpha \lambda$ ov̀

38. $T \sigma \iota \pi \lambda \alpha \chi \lambda \dot{\eta} \ddot{\eta} T \zeta \eta \pi \lambda \alpha \chi \lambda \dot{\eta}$

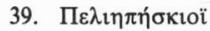

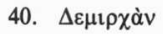

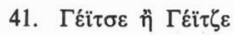

42. $\mathrm{K} \alpha \mu \iota \sigma \lambda \tilde{\eta}$

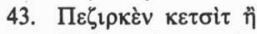

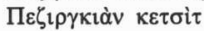

44. $\Delta \mathrm{i} \beta \mathbf{i ̀ \kappa}$ ฑै $\mathrm{T} \mathbf{\imath} \hat{\mathrm{i} \kappa}$

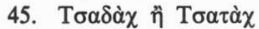

46. $\Gamma\llcorner\alpha \gamma \pi \alpha \sigma \alpha \grave{~}$

47. 'A $А \tilde{n} \Sigma \circ \varphi \tilde{\eta}$

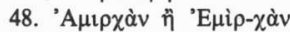

49. Гह́ï $\sigma \alpha$

50. Z

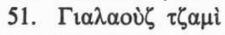

$\begin{array}{rr}70 & 263 \\ 115 & 543 \\ 45 & 511 \\ 75 & 320\end{array}$

100

120

50

80

90

70

70

70.650

$90 \quad 623$

$110 \quad 698$

$70 \quad 500$

$60 \quad 578$

$85 \quad 582$

$60 \quad 570$

75

80

$75 \quad 550$

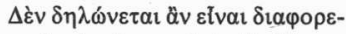

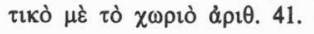

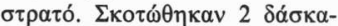

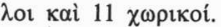

$77^{\prime} \mathrm{E} \mu \varepsilon \iota v \alpha \nu . \Sigma \kappa о \tau \omega \theta \eta \kappa \alpha \nu 10 " \mathrm{E} \lambda$ -

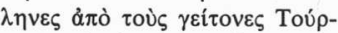

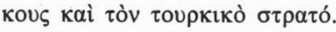

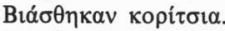

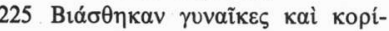

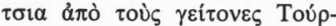

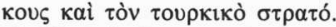

355 Tà

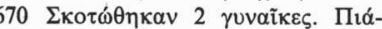

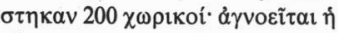

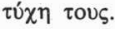

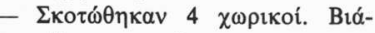

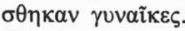

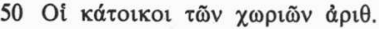

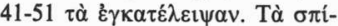

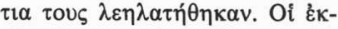

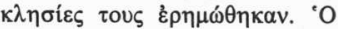

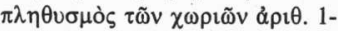

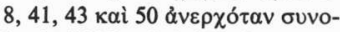

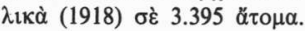

-

